


\section{Neue kovalent und ionogen aufgebaute Alkinylderivate mit Elementen der \\ 13. Gruppe des Periodensystems}

Dissertation

zur Erlangung des Doktorgrades

der Mathematisch-Naturwissenschaftlichen Fakultäten der Georg-August-Universität zu Göttingen

vorgelegt von

Marcus Schiefer

aus Hamburg

Göttingen 2002 
Referent:

Korreferent:

Tag der mündlichen Prüfung:
Prof. Dr. Dr. h. c. mult. H. W. Roesky Prof. Dr. J. Magull

31.10 .2002 
meinen Eltern und Alexandra 
Die vorliegende Dissertation wurde in der Zeit von September 1999 bis Juli 2002 unter Anleitung von Prof. Dr. Dr. h. c. mult. H. W. Roesky am Institut für Anorganische Chemie der Georg-August-Universität zu Göttingen angefertigt.

Meinem sehr verehrten Lehrer

\section{Herrn Prof. Dr. Dr. h. c. mult. H. W. Roesky}

danke ich sehr herzlich für die anregenden und offenen Diskussionen, sein Interesse am Fortgang dieser Arbeit und den vielen gewährten Freiheiten. All das war die Basis für eine stets motivierende Arbeitsatmosphäre.

Bei den Damen und Herren der Spektroskopie- und Serviceabteilungen sowie allen weiteren Institutsangehörigen bedanke ich mich für die Hilfsbereitschaft. Für die Durchführung der Einkristallröntgenstrukturanalysen danke ich besonders T. Labahn, D. Vidovic, A.- C. Schlicker, sowie H.- G. Schmidt und Dr. M. Noltemeyer für ihr großes Engagement. Herrn J. Schimkowiak und Herrn M. Schlote danke ich für die Bereitstellung der Chemikalien und des Materials.

Meinen Kolleginnen und Kollegen des Instituts danke ich für das gute Arbeitsklima und die guten Kooperationen. Besonders freundschaftlicher Dank gebührt H. Hatop, C. Cui, D. Nelke, J. Janssen, T. Blunck, A. Stasch, M. Gorol, P. Lobinger und B. Kracke. Für die seelische Unterstützung danke ich Sören und Chrischi.

Mein herzlicher Dank gilt J. Janssen, Chrischi, T. Blunck und Dr. M. Witt und allen anderen Helfern für das Korrekturlesen dieser Arbeit.

Ganz besonderer Dank gebührt meiner Familie, meinen Eltern sowie Alexandra, für die liebevolle Unterstützung und Zuwendung, die weit über materielle Unterstützung hinausging und die erfolgreiche Durchführung dieser Arbeit erst ermöglichte. 


\section{Verzeichnis der verwendeten Abkürzungen}

Abb. Abbildung

Äq. Äquivalent(e)

ATI aminotroponiminates

ax axial

ber. berechnet

br breites Signal

bzw. beziehungsweise

$\mathrm{Cp} \quad$ Cyclopentadienyl

Cp* substituiertes Cyclopentadienyl

d $\quad \operatorname{Tag}(\mathrm{e})$

$\delta \quad$ chemische Verschiebung

d. h. das heißt

Dipp 2,6-Diisopropylphenyl

DME 1,2-Dimethoxyethan

$\varnothing$ durchschnittlich

EA Elementaranalyse

EI Elektronenstoß-Ionisation

ESI Elektronenspray-Ionisation

Et Ethyl

FAB Fast-Atom-Bombardement

exc. Überschuss

gef. gefunden

(C) Grad (Celsius)

h Stunde(n)

HO Hybrid- Orbital

HSAB principle of hard and soft acids and bases

$\mathrm{Hz} \quad \mathrm{Hertz}$

$\eta \quad$ Haptizität

$i \mathrm{Bu} \quad$ iso-Butyl

$i \operatorname{Pr} \quad$ iso-Propyl

IR Infrarot

$J \quad$ Kopplungskonstante (in $\mathrm{Hz}$ ) 
K Kelvin

Kap. Kapitel

M molar

M Molekülion

Me Methyl

$\mathrm{MHz} \quad$ Megahertz

$\min \quad$ Minute(n)

MS Massenspektrum

$m / z \quad$ Masse-Ladungs-Verhältnis

$\mu \quad$ verbrückt

NMR kernmagnetische Resonanz

$\mathrm{Ph} \quad$ Phenyl

ppm parts per million

py Pyridin

pz Pyrazolat

$\mathrm{R} \quad$ 2,6-i $\mathrm{Pr}_{2} \mathrm{C}_{6} \mathrm{H}_{3} \mathrm{~N}\left(\mathrm{SiMe}_{3}\right)$ (wenn nicht anders definiert)

RT Raumtemperatur

Smp. Schmelzpunkt

Tab. Tabelle

$t \mathrm{Bu} \quad$ tertiär-Butyl

THF Tetrahydrofuran

tmp Tetramethylpyridin

TMS Tetramethylsilan

TMSCl Trimethylchlorsilan

Z Zahl der Moleküle in der Elementarzelle des betreffenden Kristalls

Zersp. Zersetzungspunkt 


\section{Einleitung}

1.1. Aufgabenstellung

\section{Theoretischer Teil}

2.1. Darstellung eines Aminoalanats und dessen Umsetzung mit THF

2.1.1. Darstellung von $\left[\mathrm{Li} \cdot \mathrm{Et}_{2} \mathrm{O}\left(2,6-i \mathrm{Pr}_{2} \mathrm{C}_{6} \mathrm{H}_{3} \mathrm{~N}\left(\mathrm{SiMe}_{3}\right) \mathrm{AlH}_{3}\right)\right]_{2}$

2.1.2. Darstellung von $\left[2,6-i \mathrm{Pr}_{2} \mathrm{C}_{6} \mathrm{H}_{3} \mathrm{~N}\left(\mathrm{SiMe}_{3}\right) \mathrm{AlOC}_{4} \mathrm{H}_{9}\right]_{2}$ (2)

2.2. Umsetzung von Alkinyl-substituierten Titano- bzw. ZirconocenKomplexen mit reaktiven Aluminiumverbindungen

2.2.1. Reaktion von reaktiven Aluminiumverbindungen mit $\mathrm{Cp}_{2} \mathrm{Ti}(\mathrm{C} \equiv \mathrm{CR})_{2}$ $(\mathrm{R}=\mathrm{Ph}, t \mathrm{Bu}, \mathrm{TMS})$

2.2.2. Reaktionen reaktiver Aluminiumverbindungen mit $\mathrm{Cp}_{2} \mathrm{Zr}(\mathrm{C} \equiv \mathrm{CPh})_{2}$ und $\mathrm{Cp}_{2}{ }_{2} \mathrm{Zr}(\mathrm{C} \equiv \mathrm{CPh})_{2}$

2.3. Darstellung einer neuen Klasse von Aluminium-Verbindungen des Typs $\left[\operatorname{RAl}(\mathrm{C} \equiv \mathrm{CR})_{3}\right]^{-} \mathrm{M}^{+}$mit drei terminalen Ethinyl-Gruppen und deren Interaktion mit Alkalimetall-Kationen

2.3.1. Einleitung

2.3.2. Darstellung von $\left[2,6-i \mathrm{Pr}_{2} \mathrm{C}_{6} \mathrm{H}_{3} \mathrm{~N}\left(\mathrm{SiMe}_{3}\right) \mathrm{AlCl}_{2}\right]_{2}$ (3)

2.3.3. Darstellung von $\left[\mathrm{K} \cdot \mathrm{THF}\left(2,6-i \mathrm{Pr}_{2} \mathrm{C}_{6} \mathrm{H}_{3} \mathrm{~N}\left(\mathrm{SiMe}_{3}\right) \mathrm{Al}(\mathrm{C} \equiv \mathrm{CPh})_{3}\right)\right]_{2}$ (4) und von $\left[\mathrm{Na} \cdot \mathrm{THF}\left(2,6-i \mathrm{Pr}_{2} \mathrm{C}_{6} \mathrm{H}_{3} \mathrm{~N}\left(\mathrm{SiMe}_{3}\right) \mathrm{Al}(\mathrm{C} \equiv \mathrm{CPh})_{3}\right)\right]_{2}(\mathbf{5})$

2.3.4. Darstellung von

$\left[\mathrm{Li} \cdot \operatorname{Dioxan}\left(2,6-i \mathrm{Pr}_{2} \mathrm{C}_{6} \mathrm{H}_{3} \mathrm{~N}\left(\mathrm{SiMe}_{3}\right) \mathrm{Al}(\mathrm{C} \equiv \mathrm{CPh})_{3}\right)\right]_{2} \cdot 2$ Dioxan $(6)$

2.3.5. Diskussion der Verbindungen $\mathbf{4}, \mathbf{5}$ und $\mathbf{6}$.

2.4. Darstellung einer neuen Klasse von Verbindungen der 13. Gruppe des Periodensystems mit drei terminalen TrimethylsilylethinylLiganden des Typs $\left[\mathrm{RM}\left(\mathrm{C} \equiv \mathrm{CSiMe}_{3}\right)_{3}\right]^{-}$

2.4.1. Darstellung von (7) $\left[\mathrm{Li} \cdot 2\right.$ Dioxan $\left.\left(2,6-i \mathrm{Pr}_{2} \mathrm{C}_{6} \mathrm{H}_{3} \mathrm{~N}\left(\mathrm{SiMe}_{3}\right) \mathrm{Al}\left(\mathrm{C} \equiv \mathrm{CSiMe}_{3}\right)_{3}\right)\right] \cdot 0.75$ Dioxan 
2.4.2. Versuche zur Darstellung von $\mathrm{RAl}\left(\mathrm{C} \equiv \mathrm{CSiMe}_{3}\right)_{2}$

$$
\left(\mathrm{R}=2,6-i \mathrm{Pr}_{2} \mathrm{C}_{6} \mathrm{H}_{3} \mathrm{~N}\left(\mathrm{SiMe}_{3}\right)\right)
$$

2.4.3. Darstellung von $\left[\mathrm{Li} \cdot \mathrm{Et}_{2} \mathrm{O}\left(2,6-i \mathrm{Pr}_{2} \mathrm{C}_{6} \mathrm{H}_{3} \mathrm{~N}\left(\mathrm{SiMe}_{3}\right) \mathrm{GaCl}_{3}\right)\right]$ (8)

2.4.4. Darstellung von $\left[(\mathrm{Li})_{2} \cdot(\text { Dioxan })_{7}\right]_{0.5}$ $\left[2,6-i \mathrm{Pr}_{2} \mathrm{C}_{6} \mathrm{H}_{3} \mathrm{~N}\left(\mathrm{SiMe}_{3}\right) \mathrm{Ga}\left(\mathrm{C} \equiv \mathrm{CSiMe}_{3}\right)_{3}\right] \cdot 1.5$ Dioxan $(9)$

2.4.5. Darstellung von

$\left[(\mathrm{Li})_{2} \cdot(\text { Dioxan })_{7}\right]_{0.5}\left[2,6-i \mathrm{Pr}_{2} \mathrm{C}_{6} \mathrm{H}_{3} \mathrm{~N}\left(\mathrm{SiMe}_{3}\right) \operatorname{In}\left(\mathrm{C} \equiv \mathrm{CSiMe}_{3}\right)_{3}\right] \cdot 1.5$ Dioxan

2.4.6. Darstellung der Verbindung

$\left[\mathrm{Li} \cdot 2 \mathrm{THF}\left(2,6-i \mathrm{Pr}_{2} \mathrm{C}_{6} \mathrm{H}_{3} \mathrm{~N}\left(\mathrm{SiMe}_{3}\right) \operatorname{In}\left(\mathrm{C} \equiv \mathrm{CSiMe}_{3}\right)_{3}\right)\right](\mathbf{1 1})$

2.5. Darstellung von Metall-Halogen-Diethinyl-Verbindungen der 13 .

Gruppe des Periodensystems des Typs $\mathrm{X}-\mathrm{M}(\mathrm{C} \equiv \mathrm{C} t \mathrm{Bu})_{2} \cdot$ n S $(\mathrm{M}=\mathrm{Al}$, Ga, In; X = Cl, Br;

$\mathrm{S}=\mathrm{THF} ; \mathrm{n}=\mathbf{1 , 2})$

2.5.1. Einleitung

2.5.2. Darstellung von $\mathrm{Br}-\mathrm{Al}(\mathrm{C} \equiv \mathrm{C} t \mathrm{Bu})_{2} \cdot 2 \mathrm{THF}$ (12)

2.5.3. Darstellung von $\mathrm{Cl}-\mathrm{Ga}(\mathrm{C} \equiv \mathrm{C} t \mathrm{Bu})_{2} \cdot \mathrm{THF}(\mathbf{1 3})$

2.5.4. Darstellung von $\mathrm{Cl}-\mathrm{In}(\mathrm{C} \equiv \mathrm{C} t \mathrm{Bu})_{2} \cdot 2 \mathrm{THF}$ (14)

2.5.5. Reaktionsverhalten der Verbindungen 12, 13 und 14

2.6. Darstellung von Metall-Triethinyl-Verbindungen der 13. Gruppe des Periodensystems des Typs $\mathrm{M}(\mathrm{C} \equiv \mathrm{C} t \mathrm{Bu})_{3} \cdot \mathrm{S}(\mathrm{M}=\mathrm{Al}, \mathrm{Ga}, \mathrm{In} ; \mathrm{S}=$ Dioxan, NEt $_{3}$ )

2.6.1. Einleitung

2.6.2. Darstellung von $\mathrm{Al}(\mathrm{C} \equiv \mathrm{C} t \mathrm{Bu})_{3} \cdot \operatorname{Dioxan}(\mathbf{1 6})$

2.6.3. Darstellung von $\mathrm{Ga}(\mathrm{C} \equiv \mathrm{C} t \mathrm{Bu})_{3} \cdot \operatorname{Dioxan}(\mathbf{1 7})$

2.6.4. Darstellung von $\operatorname{In}(\mathrm{C} \equiv \mathrm{CtBu})_{3} \cdot \mathrm{NEt}_{3}(\mathbf{1 8})$

2.7. Darstellung von Metall-Tetraethinyl-Verbindungen des Typs $\left[\mathrm{Li} \cdot \mathrm{n}\right.$ THF $\left(\mathrm{Al}(\mathrm{C} \equiv \mathrm{R})_{4}\right](\mathrm{M}=\mathrm{Al})$ 
2.8. Umsetzung der Verbindungen $4,15,19$ und $[\mathrm{Li} \cdot 2 \mathrm{THF}$

$\left.\left(\mathrm{Al}(\mathrm{C} \equiv \mathrm{CtBu})_{4}\right)\right]$ mit $\mathrm{Cp}_{2} \mathrm{ZrMe}_{2}$

2.8.1. Umsetzung von ionogenen Aluminium-Ethinyl-Verbindungen mit $\mathrm{Cp}_{2} \mathrm{ZrMe}_{2}$

2.8.2. Umsetzung mit neutralen Aluminium-Ethinyl-Verbindungen mit $\mathrm{Cp}_{2} \mathrm{ZrMe}_{2}$ zu Cp${ }_{2} \mathrm{Zr}(\mathrm{C} \equiv \mathrm{C} t \mathrm{Bu})_{2}$ (20)

2.9. Darstellung einer tetrameren Aluminium(+I)-Verbindung

2.9.1. Darstellung von $\left[\mathrm{RAll}_{2}\right]_{2}(\mathbf{2 1})\left(\mathrm{R}=2,6-i \mathrm{Pr}_{2} \mathrm{C}_{6} \mathrm{H}_{3} \mathrm{~N}\left(\mathrm{SiMe}_{3}\right)\right)$

2.9.2. Darstellung der tetrameren Aluminium(+I)-Verbindung $[\mathrm{RAl}]_{4} \cdot \operatorname{Hexan}(\mathbf{2 2}) \quad\left(\mathrm{R}=2,6-i \mathrm{Pr}_{2} \mathrm{C}_{6} \mathrm{H}_{3} \mathrm{~N}\left(\mathrm{SiMe}_{3}\right)\right)$

4.1. Allgemeine Arbeitstechniken und Messmethoden

4.3. Darstellung eines Aminoalans und dessen Umsetzung mit THF

4.3.1. Darstellung von $\left[\mathrm{Li} \cdot \mathrm{Et}_{2} \mathrm{O}\left(2,6-i \mathrm{Pr}_{2} \mathrm{C}_{6} \mathrm{H}_{3} \mathrm{~N}\left(\mathrm{SiMe}_{3}\right) \mathrm{AlH}_{3}\right)\right]_{2} \quad$ (1)

4.3.2. Darstellung von $\left[2,6-i \mathrm{Pr}_{2} \mathrm{C}_{6} \mathrm{H}_{3} \mathrm{~N}\left(\mathrm{SiMe}_{3}\right) \mathrm{AlOC}_{4} \mathrm{H}_{9}\right]_{2}$ (2)

4.4. Umsetzung von Alkinyl-substituierten Titano- bzw. ZirconocenKomplexen mit reaktiven Aluminiumverbindungen

4.4.1. Reaktion von reaktiven Aluminiumverbindungen mit $\mathrm{Cp}_{2} \mathrm{Ti}(\mathrm{C} \equiv \mathrm{CR})_{2}$ $(\mathrm{R}=\mathrm{Ph}, t \mathrm{Bu})$

4.5. Darstellung einer neuen Klasse von Aluminium-Verbindungen des Typs $\left[\mathrm{RAI}(\mathrm{C} \equiv \mathrm{CR})_{3}\right]^{-} \mathrm{M}^{+}$mit drei terminalen Ethinyl-Gruppen und deren Interaktion mit Alkalimetall-Kationen 
4.5.2. Darstellung von $\left[\mathrm{K} \cdot \operatorname{THF}\left(2,6-i \mathrm{Pr}_{2} \mathrm{C}_{6} \mathrm{H}_{3} \mathrm{~N}\left(\mathrm{SiMe}_{3}\right) \mathrm{Al}(\mathrm{C} \equiv \mathrm{CPh})_{3}\right)\right]_{2}(4)$

4.5.3. Darstellung von $\left[\mathrm{Na} \cdot \mathrm{THF}\left(2,6-i \mathrm{Pr}_{2} \mathrm{C}_{6} \mathrm{H}_{3} \mathrm{~N}\left(\mathrm{SiMe}_{3}\right) \mathrm{Al}(\mathrm{C} \equiv \mathrm{CPh})_{3}\right)\right]_{2}(\mathbf{5})$

4.5.4. Darstellung von $\left[\mathrm{Li} \cdot \operatorname{Dioxan}\left(2,6-i \mathrm{Pr}_{2} \mathrm{C}_{6} \mathrm{H}_{3} \mathrm{~N}\left(\mathrm{SiMe}_{3}\right) \mathrm{Al}(\mathrm{C} \equiv \mathrm{CPh})_{3}\right)\right]_{2} \cdot 2$ Dioxan (6)

4.6. Darstellung einer neuen Klasse von Verbindungen der 13. Gruppe des Periodensystems mit drei terminalen TrimethylsilylethinylLiganden des Typs $\left[\mathrm{RM}\left(\mathrm{C} \equiv \mathrm{CSiMe}_{3}\right)_{3}\right]^{-}$

4.6.1. Darstellung von

$\left[\mathrm{Li} \cdot 2\right.$ Dioxan $\left.\left(2,6-i \mathrm{Pr}_{2} \mathrm{C}_{6} \mathrm{H}_{3} \mathrm{~N}\left(\mathrm{SiMe}_{3}\right) \mathrm{Al}\left(\mathrm{C} \equiv \mathrm{CSiMe}_{3}\right)_{3}\right)\right] \cdot 0.75$ Dioxan (7)

4.6.2. Versuche zur Darstellung von $\mathrm{RAl}\left(\mathrm{C} \equiv \mathrm{CSiMe}_{3}\right)_{2}$

$$
\left(\mathrm{R}=2,6-i \mathrm{Pr}_{2} \mathrm{C}_{6} \mathrm{H}_{3} \mathrm{~N}\left(\mathrm{SiMe}_{3}\right)\right) \quad 77
$$

4.6.3. Darstellung von $\left[\mathrm{Li} \cdot \mathrm{Et}_{2} \mathrm{O}\left(2,6-i \mathrm{Pr}_{2} \mathrm{C}_{6} \mathrm{H}_{3} \mathrm{~N}\left(\mathrm{SiMe}_{3}\right) \mathrm{GaCl}_{3}\right)\right]$ (8) 78

4.6.4. Darstellung von

$\left[(\mathrm{Li})_{2} \cdot(\text { Dioxan })_{7}\right]_{0.5}\left[2,6-i \mathrm{Pr}_{2} \mathrm{C}_{6} \mathrm{H}_{3} \mathrm{~N}\left(\mathrm{SiMe}_{3}\right) \mathrm{Ga}\left(\mathrm{C} \equiv \mathrm{CSiMe}_{3}\right)_{3}\right] \cdot(\text { Dioxan })_{1.5}$

4.6.5. Darstellung von

$\left[(\mathrm{Li})_{2} \cdot(\text { Dioxan })_{7}\right]_{0.5}\left[2,6-i \operatorname{Pr}_{2} \mathrm{C}_{6} \mathrm{H}_{3} \mathrm{~N}\left(\mathrm{SiMe}_{3}\right) \mathrm{In}\left(\mathrm{C} \equiv \mathrm{CSiMe}_{3}\right)_{3}\right] \cdot(\text { Dioxan })_{1.5}$

4.6.6. Darstellung der Verbindung

$\left[\mathrm{Li} \cdot 2 \mathrm{THF}\left(2,6-i \mathrm{Pr}_{2} \mathrm{C}_{6} \mathrm{H}_{3} \mathrm{~N}\left(\mathrm{SiMe}_{3}\right) \operatorname{In}\left(\mathrm{C} \equiv \mathrm{CSiMe}_{3}\right)_{3}\right)\right] \quad$ (11)

4.7. Darstellung von Metall-Halogen-Diethinyl-Verbindungen der 13.

Gruppe des Periodensystems des Typs $\mathrm{X}-\mathrm{M}(\mathrm{C} \equiv \mathrm{C} t \mathrm{Bu})_{2} \cdot$ n S $(\mathrm{M}=\mathrm{Al}$,

Ga, In; X = Cl, Br; S = THF; $n=1,2)$

4.7.1. Darstellung von $\mathrm{Br}-\mathrm{Al}(\mathrm{C} \equiv \mathrm{C} t \mathrm{Bu})_{2} \cdot 2 \mathrm{THF}$ (12)

4.7.2. Darstellung von $\mathrm{Cl}-\mathrm{Ga}(\mathrm{C} \equiv \mathrm{C} t \mathrm{Bu})_{2} \cdot \mathrm{THF}(\mathbf{1 3})$

4.7.3. Darstellung von $\mathrm{Cl}-\mathrm{In}(\mathrm{C} \equiv \mathrm{C} t \mathrm{Bu})_{2} \cdot 2 \mathrm{THF}$ (14)

4.7.4. Reaktionsverhalten der Verbindungen 12, 13 und 14

4.8. Darstellung von Metall-Triethinyl-Verbindungen der 13. Gruppe des Periodensystems des Typs $\mathrm{M}(\mathrm{C} \equiv \mathrm{C} t \mathrm{Bu})_{3} \cdot \mathrm{S}(\mathrm{M}=\mathrm{Al}, \mathrm{Ga}, \mathrm{In} ; \mathrm{S}=$ Dioxan, $\mathbf{N E t}_{3}$ )

4.8.1. Darstellung von $\mathrm{Al}(\mathrm{C} \equiv \mathrm{C} t \mathrm{Bu})_{3} \cdot \operatorname{Dioxan}(\mathbf{1 6})$ 
4.8.2. Darstellung von $\mathrm{Ga}(\mathrm{C} \equiv \mathrm{C} t \mathrm{Bu})_{3} \cdot \operatorname{Dioxan}(\mathbf{1 7})$

4.8.3. Darstellung von $\operatorname{In}(\mathrm{C} \equiv \mathrm{C} t \mathrm{Bu})_{3} \cdot \mathrm{NEt}_{3}(\mathbf{1 8})$

4.9. Darstellung von Aluminium-Tetraethinyl-Verbindungen des Typs $\left[\mathbf{L i}\left(\mathrm{Al}(\mathrm{C} \equiv \mathbf{R})_{4}\right)\right] \cdot \mathbf{T H F} \quad 85$

4.9.1. Darstellung von $\left[\mathrm{Li} \cdot \mathrm{THF}\left(\mathrm{Al}(\mathrm{C} \equiv \mathrm{C} t \mathrm{Bu})_{4}\right)\right](\mathbf{1 9})$

4.10. Umsetzung von Metall-Ethinyl-Verbindungen mit $\mathrm{Cp}_{2} \mathrm{ZrMe}_{2} \quad 86$

4.10.1. Umsetzung von $\mathrm{Al}(\mathrm{C} \equiv \mathrm{C} t \mathrm{Bu})_{3} \cdot$ Dioxan mit $\mathrm{Cp}_{2} \mathrm{ZrMe}_{2}$

4.11. Darstellung einer tetrameren Aluminium(+I)-Verbindung 86

4.11.1. Darstellung von $\left[\mathrm{RAlI}_{2}\right]_{2}(\mathbf{2 1})\left(\mathrm{R}=2,6-i \mathrm{Pr}_{2} \mathrm{C}_{6} \mathrm{H}_{3} \mathrm{~N}\left(\mathrm{SiMe}_{3}\right)\right) \quad 86$

4.11.2. Darstellung der tetrameren Aluminium(+I)-Verbindung

$[\mathrm{RAl}]_{4} \cdot \operatorname{Hexan}(\mathbf{2 2}) \quad\left(\mathrm{R}=2,6-i \mathrm{Pr}_{2} \mathrm{C}_{6} \mathrm{H}_{3} \mathrm{~N}\left(\mathrm{SiMe}_{3}\right)\right)$

5. Röntgenographischer Anhang

6. Literaturverzeichnis 


\section{Einleitung}

Carbonylolefinierungen, d. h. die Umwandlung von Aldehyden und Ketonen in Alkene, sind in der Synthese sehr wichtige Prozesse und können mit Hilfe der Wittig-Reaktion bewerkstelligt werden. ${ }^{[1,2,3]}$

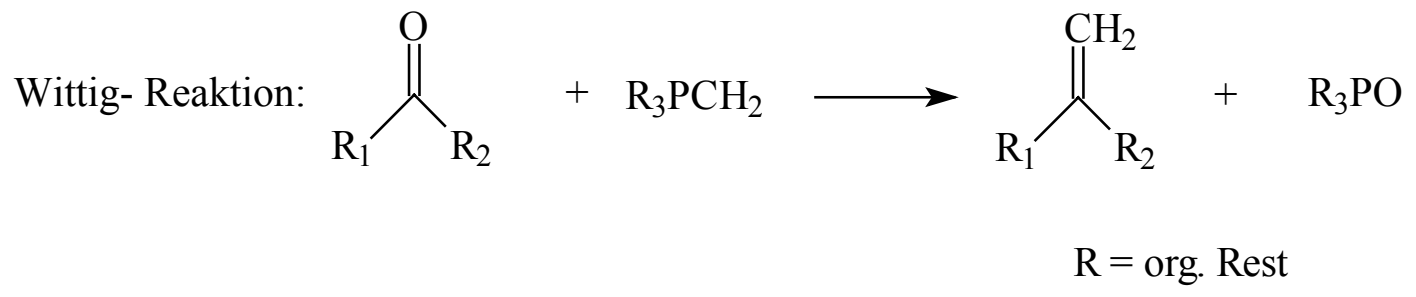

Schema 1: Wittig- Reaktion.

Carbonsäureester und -amide können mit dieser Methode allerdings nicht olefiniert werden, denn ihre Carbonylreaktivität ist hierfür normalerweise zu gering. ${ }^{[4]}$

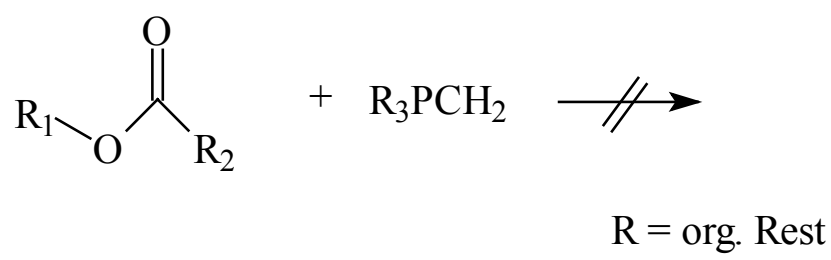

Schema 2: Grenzen der Wittig-Reaktion.

Für diese Umsetzung bedarf es also eines reaktiveren Olefinierungsreagenzes, welches in dem "Tebbe-Reagenz" gefunden wurde. Dieses wird aus $\mathrm{Cp}_{2} \mathrm{TiCl}_{2}$ und $\mathrm{AlMe}_{3}$ unter Methanabspaltung gewonnen. ${ }^{[5,6]}$

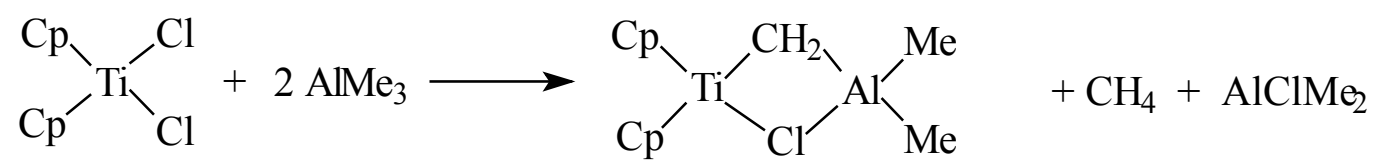

Schema 3: Bildung des Tebbe- Reagenzes.

Unter Zusatz von Lewis- Basen reagiert das Tebbe- Reagenz unter C-C-Verknüpfung zu dem gewünschten Olefin: ${ }^{[7]}$

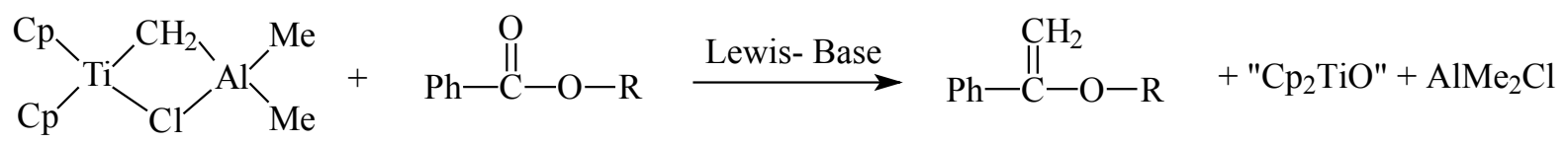

Schema 4: Reaktion des Tebbe-Reagenzes. 
Der Zusatz von Lewis-Basen beschleunigt die Reaktion sehr stark, da die Generierung des reaktiven Intermediates $" \mathrm{Cp}_{2} \mathrm{Ti}=\mathrm{CH}_{2}$ " begünstigt wird. Dieses Intermediat wird auch durch das "Grubbs-Reagenz" gebildet. ${ }^{[8]}$

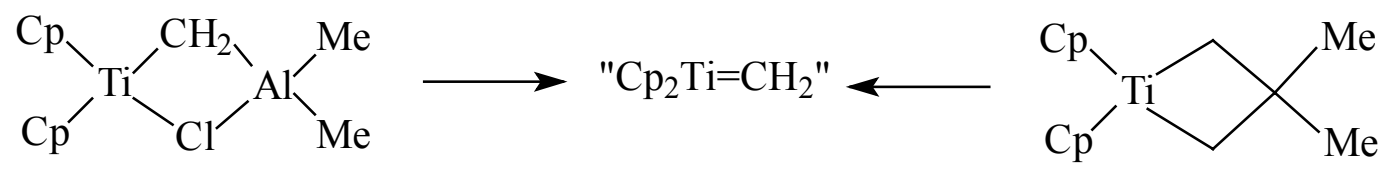

Schema 5: Bildung des reaktiven Intermediates.

Das Tebbe- sowie das Grubbs-Reagenz haben jedoch den Nachteil, dass sie lediglich eine Methylengruppe übertragen können aber keine größeren Reste.

Wenn sich ein reaktives Intermediat der Form " $\mathrm{Cp}_{2} \mathrm{Ti}=\mathrm{C}=\mathrm{CR}_{2}$ " bildet, sollten bei der Reaktion mit Ketonen Allene entstehen. Dies konnte durch die folgende Reaktion gezeigt werden: ${ }^{[9]}$

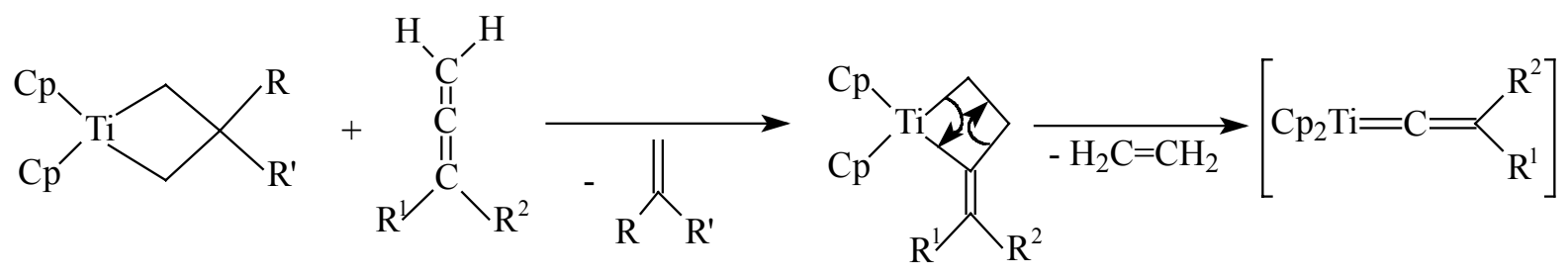

Schema 6: Bildung des reaktiven Intermediats zur Übertragung von Allen-Gruppen.<smiles>[R]C(=O)CCC</smiles>

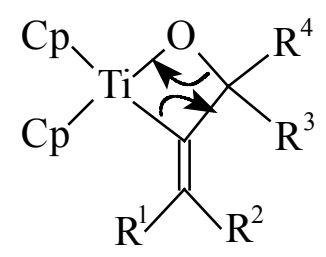<smiles>[R]C([R])=C=C([R])[R]</smiles>

Schema 7: Reaktion des reaktiven Intermediats mit einer Carbonyl-Verbindung.

Der große Nachteil dieser Reaktion ist, dass der Darstellung eines substituierten Allens aus einem reaktiven Intermediat der Einsatz eines Allens voraus geht.

Allene sind für die organische Synthese von großer Bedeutung, deren Darstellung ist jedoch aufwändig. ${ }^{[10]}$ Würde man ein Vinyliden-Derivat auf der Basis von Titan und Aluminium als 
Reaktionspartner eines Ketons bzw. Aldehyds einsetzen, sollte die Bildung des reaktiven Reagenzes die Folge sein, welches mit Ketonen das gewünschte prochirale Allen bildet:

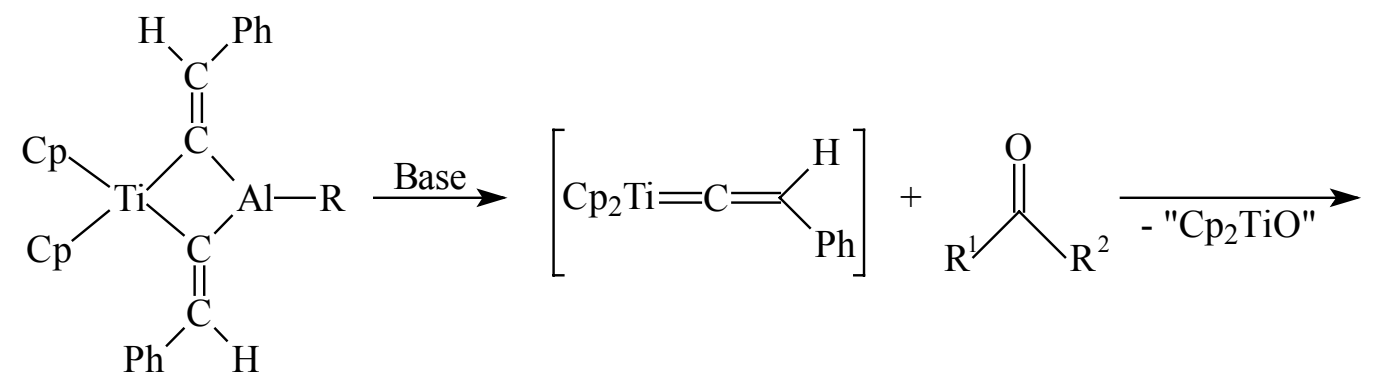<smiles>[R]C([R])=C=Cc1ccccc1</smiles>

Schema 8: Erwartete Reaktion eines Titan-Aluminium-Vinylidens mit Ketonen.

Um zu dem gewünschten Titan-Aluminium-Vinyliden-Derivat zu gelangen, sollte es zwei verschiedene Wege geben:

1. Weg: Die Reaktion von bekannten Übergangsmetallocen-Diethinyl-Verbindungen mit reaktiven Aluminium-Verbindungen, wie z. B. monomeren AluminiumDihydriden.<smiles>C#C[I-](C#C)(C#Cc1ccccc1)C1CCCCC1</smiles><smiles>[R][AlH2]</smiles><smiles></smiles>

Schema 9: Weg 1 zur Darstellung eines Vinyliden-Derivates. 
2. Weg: Die Reaktion von reaktiven Metallocen-Alkyl-Verbindungen mit Triel-DiethinylVerbindungen

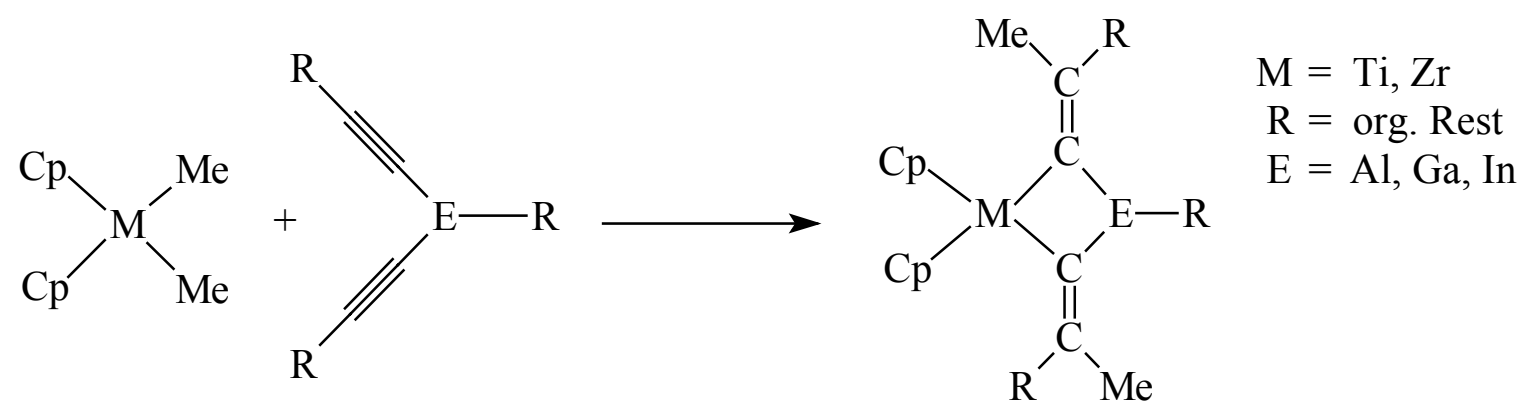

Schema 10: Weg 2 zur Darstellung eines Vinyliden-Derivates.

Der Vorteil des ersten Weges ist, dass viele Übergangsmetallocen-Diethinyl-Verbindungen bekannt und einfach darzustellen sind. Viele der ebenfalls benötigten reaktiven AluminiumVerbindungen sind in diesem Arbeitskreis entstanden und leicht zugänglich.

Setzt man entsprechend dem zweiten Weg eine Aluminium-Verbindung mit einer terminalen Ethinyl-Gruppe ein, erhält man einen ethinylverbrückten zweikernigen AluminiumZirconocen-Komplex. ${ }^{[11,12]}$

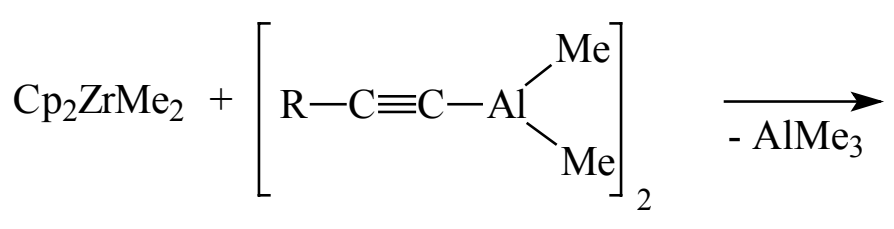<smiles></smiles>

Schema 11: Darstellung eines ethinylverbrückten zweikernigen Aluminium-Zirconocen-Komplexes.

Der Einsatz eines Triels mit zwei oder mehr terminalen Ethinyl-Gruppen anstelle von $\mathrm{Me}_{2} \mathrm{Al}-\mathrm{C} \equiv \mathrm{CPh}$ sollte zu dem gewünschten Titan-Aluminium-Vinyliden-Derivat führen. Die Alkinylchemie der 13. Gruppe des Periodensystems ist jedoch nur sehr schlecht entwickelt. So gibt es im Fall des Aluminiums und Indiums nur sehr wenige, im Fall des Galliums keine Triel-Verbindungen mit zwei oder mehr terminal gebundenen Ethinyl-Liganden. ${ }^{[13,14]}$ Vor der Durchführung des zweiten Weges sind daher grundlegende Vorarbeiten bei der Darstellung dieser Metall-Verbindungen notwendig. 


\subsection{Aufgabenstellung:}

Ziel dieser Arbeit war es, die Möglichkeiten zur Bildung eines Vinyliden-Derivates aus

a.) der Reaktion einer Metallocen-Diethinyl-Verbindung mit einer reaktiven Aluminium-Verbindung

und b.) der Reaktion einer Triel-Verbindung mit zwei oder mehr Ethinyl-Liganden mit einer Metallocen-Verbindung

zu untersuchen. 


\section{Theoretischer Teil}

\subsection{Darstellung eines Aminoalans und dessen Umsetzung mit THF}

\subsubsection{Darstellung von $\left[\mathrm{Li} \cdot \mathrm{Et}_{2} \mathrm{O}\left(2,6-i \mathrm{Pr}_{2} \mathrm{C}_{6} \mathrm{H}_{3} \mathrm{~N}\left(\mathrm{SiMe}_{3}\right) \mathrm{AlH}_{3}\right)\right]_{2}$}

Aluminium-Dihydride und deren Synthese sind schon seit längerer Zeit bekannt. ${ }^{[15,16,17]}$ Für die Umsetzung eines solchen Aluminium-Dihydrids mit Titanocen-Komplexen wurde eine einfach herzustellende Verbindung mit einem gut kristallisierenden Ligandensystem hergestellt.

Umsetzung von 2,6-i $\operatorname{Pr}_{2} \mathrm{C}_{6} \mathrm{H}_{3} \mathrm{~N}\left(\mathrm{SiMe}_{3}\right) \mathrm{H}$ mit $\mathrm{LiAlH}_{4}$ in Diethylether bei Raumtemperatur lieferte nach Umkristallisation 1 als einen farblosen Feststoff. Jedoch gelang es nicht, Einkristalle von 1 zu erhalten.
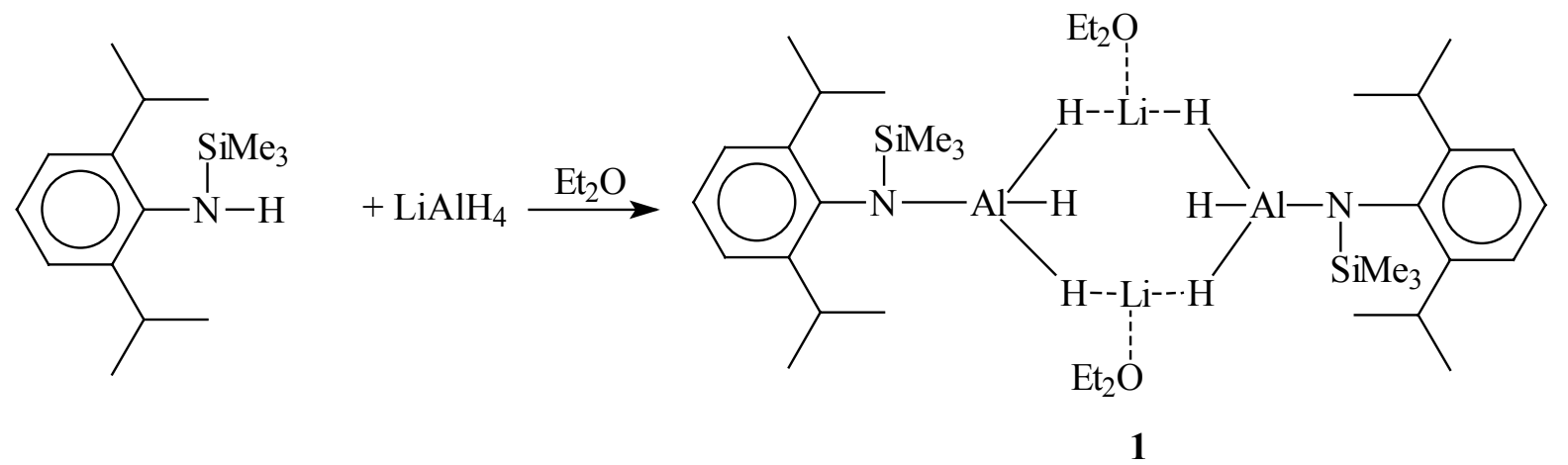

Schema 12: Darstellung von 1.

Das ${ }^{1}$ H-NMR- Spektrum zeigt bei $\delta 7.2$ - 6.9 die Resonanzen der Aryl-Protonen. Die Resonanzen der $\mathrm{CH}_{3}$ - und der $\mathrm{OCH}_{2}$ - Protonen des Diethylethers sind im Spektrum bei $\delta 0.96$ und 3.19 zu sehen. Das Spektrum zeigt außerdem die Protonen-Resonanzen der IsopropylGruppe bei $\delta 3.90(\mathrm{CH}), 1.40$ und $1.31\left(\mathrm{CH}_{3}\right)$ sowie die Protonen-Resonanzen der $\mathrm{SiMe}_{3}-$ Gruppe. Im ${ }^{7} \mathrm{Li}-\mathrm{NMR}$ - Spektrum wird die Resonanz bei $\delta$-0.46 des $\mathrm{Et}_{2} \mathrm{O}$-koordinierten Lithium-Kations beobachtet. Im IR-Spektrum ist die Absorptionsbande der Valenzschwingung der $\mathrm{AlH}_{3}$-Gruppe bei $1773 \mathrm{~cm}^{-1} \mathrm{zu}$ sehen und liegt damit im bekanten Bereich. ${ }^{[18]}$ Das Massenspektrum zeigt das Signal des $\left(2,6-i \mathrm{Pr}_{2} \mathrm{C}_{6} \mathrm{H}_{3} \mathrm{~N}\left(\mathrm{SiMe}_{3}\right) \mathrm{AlH}\right)^{+}-\mathrm{Ions}$ sowie das des $\left(2,6-i \mathrm{Pr}_{2} \mathrm{C}_{6} \mathrm{H}_{3} \mathrm{~N}\left(\mathrm{SiMe}_{3}\right)\right)^{+}$-Ions. Die Elementaranalyse bestätigt die gefundene Zusammensetzung $\mathrm{C}_{19} \mathrm{H}_{39}$ AlLiNOSi. 


\subsubsection{Darstellung von $\left[2,6-i \mathrm{Pr}_{2} \mathrm{C}_{6} \mathrm{H}_{3} \mathrm{~N}\left(\mathrm{SiMe}_{3}\right) \mathrm{AlOC}_{4} \mathrm{H}_{9}\right]_{2}$ (2)}

Aus 1 sollte zunächst das freie Aluminium-Dihydrid (2,6-i $\left.\operatorname{Pr}_{2} \mathrm{C}_{6} \mathrm{H}_{3} \mathrm{~N}\left(\mathrm{SiMe}_{3}\right) \mathrm{AlH}_{2}\right)$ dargestellt werden. Dafür wurde Verbindung 1 in Diethylether mit einem leichten Überschuss an Methyliodid versetzt und bei Raumtemperatur gerührt. Nachdem über Celite filtriert wurde, konnten die leichtflüchtigen Bestandteile im Vakuum entfernt werden. Es blieb eine zähflüssige Masse übrig, deren spektroskopische Untersuchung mittels eines ${ }^{1} \mathrm{H}-\mathrm{NMR}$ Spektrums auf das gewünschte Aluminium-Dihydrid hindeutete. Das Ausbleiben von Resonanzen im ${ }^{7}$ Li-NMR macht wahrscheinlich, dass es sich bei dem entstandenen Produkt nicht mehr um ein LiH-Addukt handelt.

Das ölige Produkt wurde in THF aufgenommen und zum Kristallisieren bei $12{ }^{\circ} \mathrm{C}$ gelagert. Es bildeten sich wenige klare Kristalle, die mit Hilfe einer Röntgenstrukturanalyse als das THFInsertionsprodukt [2,6-i $\left.i \mathrm{Pr}_{2} \mathrm{C}_{6} \mathrm{H}_{3} \mathrm{~N}\left(\mathrm{SiMe}_{3}\right) \mathrm{AlOC}_{4} \mathrm{H}_{9}\right]_{2}$ identifiziert werden konnten. Das ${ }^{1}$ H-NMR-Spektrum zeigt jedoch, dass kein einheitliches Produkt entstanden war, sondern ein Gemisch, welches weder durch Umkristallisation noch durch Sublimation getrennt werden konnte. Aufgrund der bekannten THF-Öffnungsreaktionen mit starken Lewis-Säuren ${ }^{[19,20]}$ wurde auf weiterführende Untersuchungen verzichtet.

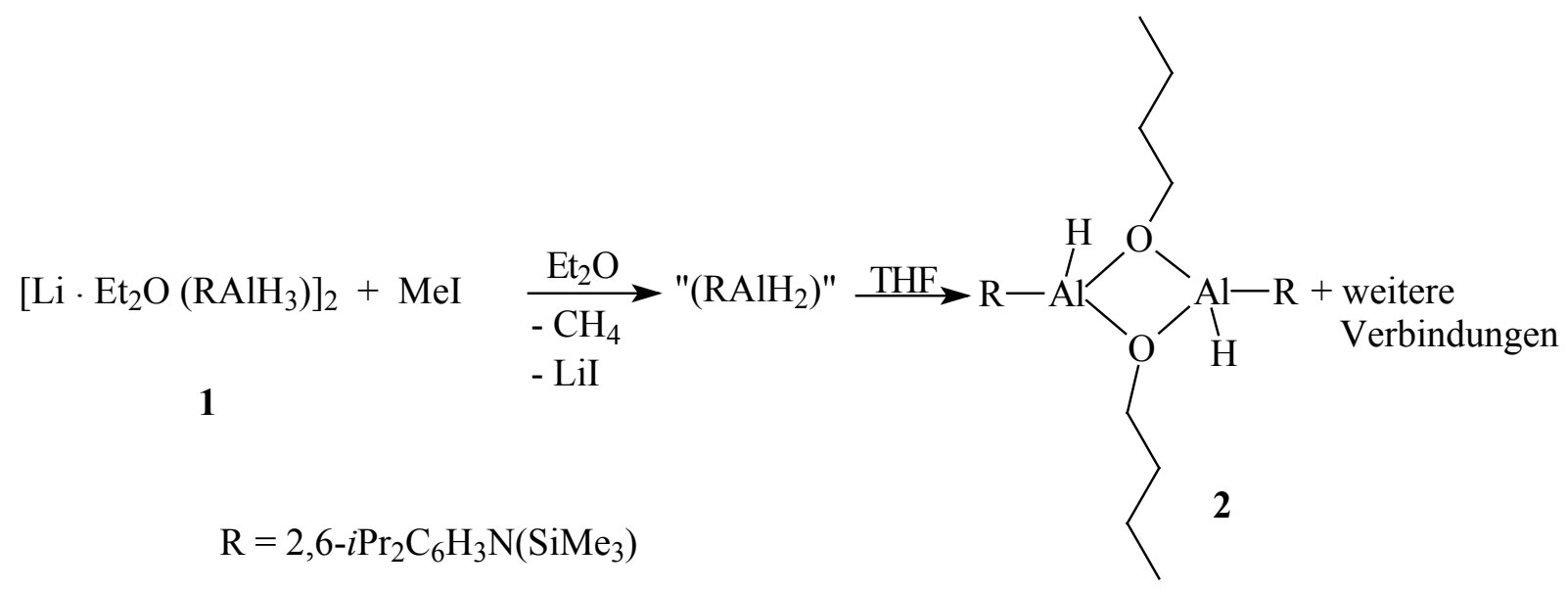

Schema 13: Identifiziertes Nebenprodukt 2.

Ausgewählte Bindungslängen $(\AA)$ und Winkel $\left(^{\circ}\right)$ für 2.

$\begin{array}{llll}\mathrm{Al}(1)-\mathrm{N}(1) & 1.812(2) & \mathrm{N}(1)-\mathrm{Al}(1)-\mathrm{O}(1) & 117.65(6) \\ \mathrm{Al}(1)-\mathrm{O}(1) \mathrm{A} & 1.8337(13) & \mathrm{O}(1) \mathrm{A}-\mathrm{Al}(1)-\mathrm{O}(1) & 80.03(6) \\ \mathrm{Al}(1)-\mathrm{O}(1) & 1.8356(13) & \mathrm{C}(1)-\mathrm{O}(1)-\mathrm{Al}(1) \mathrm{A} & 129.16(11) \\ \mathrm{O}(1)-\mathrm{C}(1) & 1.451(2) & \mathrm{C}(1)-\mathrm{O}(1)-\mathrm{Al}(1) & 128.84(11) \\ \mathrm{N}(1)-\mathrm{Al}(1)-\mathrm{O}(1) \mathrm{A} & 114.37(7) & \mathrm{Al}(1) \mathrm{A}-\mathrm{O}(1)-\mathrm{Al}(1) & 99.97(6)\end{array}$




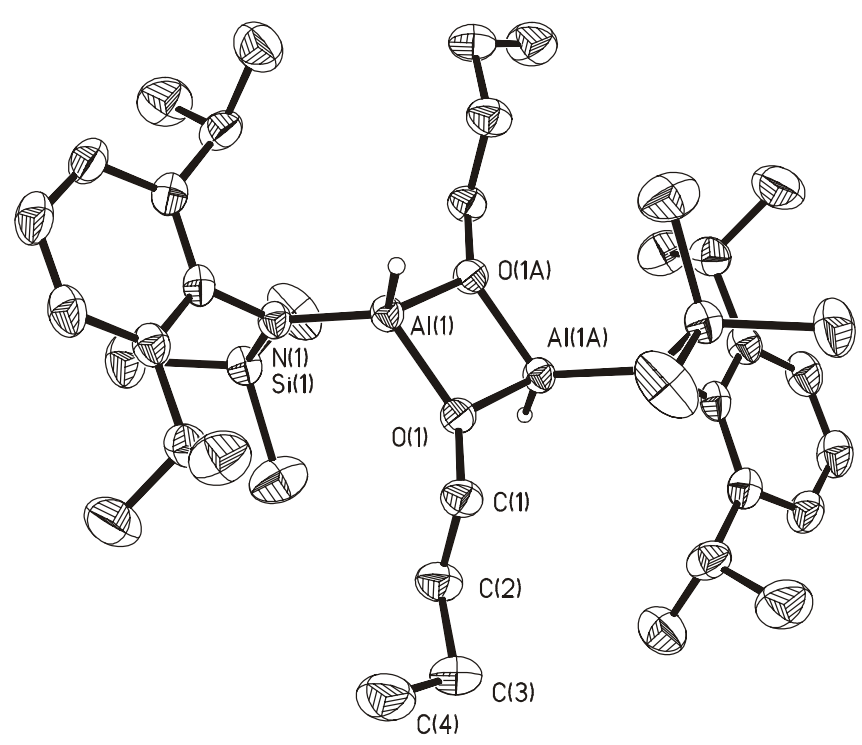

Abb. 1: Struktur von 2 im Kristall.

In Verbindung 2 liegen die Al-O-Bindungslängen des viergliedrigen Ringes (1.834 und $1.836 \AA$ ) im Bereich bekannter Bindungslängen ${ }^{[21,22,23,24,25]}$ (vergleiche auch Tab. 1). Die tetraedrische Koordination am Aluminiumatom ist stark verzerrt, so beträgt der O-Al-OBindungswinkel nur $80.03^{\circ}$, während der exocyclische N-Al-O-Winkel $117.65^{\circ}$ einnimmt. Diese Werte liegen ebenfalls im Bereich der bekannten Verbindungen (vgl. Tab. 1).

Tabelle 1: Bindungslängen und Winkel einiger vergleichbarer Verbindungen:

\begin{tabular}{|l|l|l|}
\hline & Al-O-Bindungslänge $(\AA)$ & O-Al-O-Bindungswinkel \\
\hline$\left\{\left(i \mathrm{Pr}_{2}-\mathrm{ATI}\right) \mathrm{Al}(\mu-\mathrm{O} i \mathrm{Pr})\right\}_{2}{ }^{2+[24]}$ & $1.809-1.817$ & $80.2^{\circ}$ \\
\hline$\left[\left(\mu-\mathrm{O}\left(\mathrm{CH}_{2}\right)_{2} \mathrm{OPh}\right) \mathrm{Al}(i \mathrm{Bu})_{2}\right]_{2}{ }^{[25]}$ & 1.828 & $79.6^{\circ}$ \\
\hline$\left[\left(\mu-\mathrm{O}\left(\mathrm{CH}_{2}\right)_{2} \mathrm{OPh}\right) \mathrm{Al}(\mathrm{Me})_{2}\right]_{2}{ }^{[25]}$ & 1.821 & $78.8^{\circ}$ \\
\hline
\end{tabular}

Neu an dieser Verbindung ist, dass ein Aluminiumatom des viergliedrigen Ringes neben einem stickstoffhaltigen Liganden ein Wasserstoffatom gebunden hat.

Aufgrund des Trennungsproblems sowie einer anderen Zielsetzung dieser Arbeit wurde diese Reaktion nicht weiter verfolgt. 


\subsection{Umsetzung von Alkinyl-substituierten Titano- bzw. Zirconocen- Komplexen mit reaktiven Aluminiumverbindungen}

\subsubsection{Reaktion von reaktiven Aluminiumverbindungen mit $\mathrm{Cp}_{2} \mathrm{Ti}(\mathrm{C} \equiv \mathrm{CR})_{2} \quad(\mathrm{R}=\mathrm{Ph}$, $t \mathrm{Bu})$}

Um ein Titan-Aluminium-Vinyliden-Derivat zu erhalten, wurden zahlreiche Versuche unternommen. Stets wurde der Alkinyl-substituierte Titanocen-Komplex $\mathrm{Cp}_{2} \mathrm{Ti}(\mathrm{C} \equiv \mathrm{CPh})_{2}$ in Diethylether bzw. THF gelöst. Zu dieser Lösung wurde bei $-78{ }^{\circ} \mathrm{C}$ die Lösung mit dem Aluminiumhydrid gegeben und 24 Stunden lang bei Raumtemperatur gerührt. Die erhoffte Reaktion blieb aber aus (vgl. Schema 9).

Statt dessen fiel bei allen Versuchen stets ein grüner, in polaren wie unpolaren Lösungsmitteln unlöslicher Niederschlag aus, und die Lösung färbte sich rötlich. Es zeigte sich, dass es sich bei dem Niederschlag um die bereits bekannte Titanocen(III)-Verbindung $\left[\mathrm{Cp}_{2} \mathrm{Ti}(\mathrm{C} \equiv \mathrm{CPh})\right]_{2}$ handelte. ${ }^{[26,27,28]}$

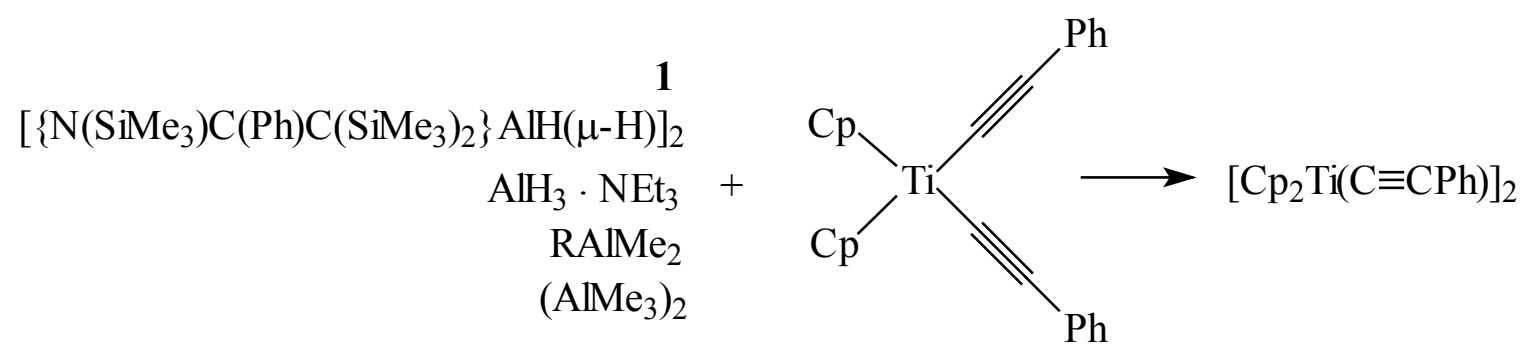

Schema 14: Umsetzung reaktiver Aluminiumverbindungen mit $\mathrm{Cp}_{2} \mathrm{Ti}(\mathrm{C} \equiv \mathrm{CPh})_{2}$. $\left(\mathrm{R}=2,6-i \mathrm{Pr}_{2} \mathrm{C}_{6} \mathrm{H}_{3} \mathrm{~N}\left(\mathrm{SiMe}_{3}\right)\right)$

Nach dem Entfernen des Lösungsmittels vom Filtrat blieb eine rötliche, zähflüssige Masse übrig, woraus weder nach Kristallisation noch Sublimation eine einheitliche Substanz isoliert und identifiziert werden konnte.

Die Variation der organischen Reste an der Ethinylgruppe führte zu keinem anderen Ergebnis. Dennoch ist der Syntheseweg für die Titanocen(III)-Ethinyl-Verbindungen neu, wurde doch sonst von Titanocen(III)-Chlorid ausgegangen, das mit einem Ethinyl- Salz versetzt wurde. ${ }^{[26]}$

$$
2 \mathrm{Cp}_{2} \mathrm{TiCl}+2 \mathrm{NaC} \equiv \mathrm{CPh} \longrightarrow\left[\mathrm{Cp}_{2} \mathrm{Ti}(\mathrm{C} \equiv \mathrm{CPh})\right]_{2}+2 \mathrm{NaCl}
$$

Schema 15: Herkömmliche Darstellung von $\left.\mathrm{Cp}_{2} \mathrm{Ti}(\mathrm{C} \equiv \mathrm{CPh})\right]_{2}$ 


\subsubsection{Reaktionen reaktiver Aluminiumverbindungen mit $\operatorname{Cp}_{2} \mathrm{Zr}(\mathrm{C} \equiv \mathrm{CPh})_{2}$ und $\mathrm{Cp}_{2}{ }_{2} \mathrm{Zr}(\mathrm{C} \equiv \mathrm{CPh})_{2}$}

Um die Reduktion des Übergangsmetalls zu verhindern, wurden Organo-ZirconocenKomplexe gewählt, da dieses Element eine deutlich schwächer ausgeprägte Neigung zur Reduktion $(\mathrm{Zr}(+\mathrm{IV}) \rightarrow \mathrm{Zr}(+\mathrm{III}))$ hat als Organo-Titan-Komplexe $(\mathrm{Ti}(+\mathrm{IV}) \rightarrow \mathrm{Ti}(+\mathrm{III}))^{[29,31]}$ (z.B. $\left[\mathrm{Cp}_{2} \mathrm{MCl}_{2}\right]+\mathrm{e}^{-} \rightarrow\left[\mathrm{Cp}_{2} \mathrm{MCl}_{2}\right]^{-}: \Delta \mathrm{E}^{\circ}$ für $\mathrm{M}=\mathrm{Zr}: 1.70 \mathrm{~V}$; Ti: $\left.0.75 \mathrm{~V}\right){ }^{[30]}$

Außerdem sind Zirconium-III-Komplexe kinetisch instabiler als ihre Titananalogen. ${ }^{[31]}$ So wurde zunächst der Alkinyl-substituierte Zirconocen-Komplex $\mathrm{Cp}_{2} \mathrm{Zr}(\mathrm{C} \equiv \mathrm{CPh})_{2}$ eingesetzt und in verschiedenen Versuchen mit reaktiven Aluminiumverbindungen versetzt. Es zeigte sich, dass der schlecht lösliche Komplex nicht mit den eingesetzten Reagentien reagierte.

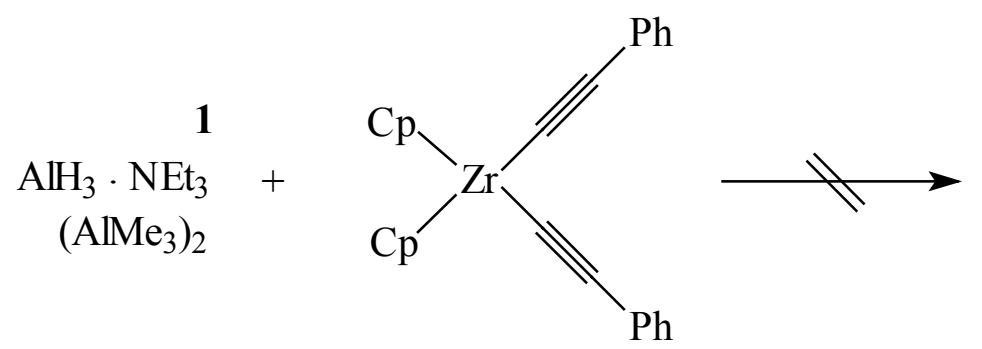

Schema 16: Misslungene Versuche zur Darstellung eines Zirconium-Aluminium-Vinyliden-Derivats.

Um auszuschließen, dass die Reaktion aufgrund der sehr schlechten Löslichkeit von $\mathrm{Cp}_{2} \mathrm{Zr}(\mathrm{C} \equiv \mathrm{CPh})_{2}$ nicht einsetzen konnte, wurde statt dessen das gut lösliche $\mathrm{Cp}_{2}{ }_{2} \mathrm{Zr}(\mathrm{C} \equiv \mathrm{CPh})_{2}$ verwendet. Hierbei stellte sich jedoch heraus, dass die Farbänderung der Reaktionslösung nicht auf die Zugabe der Aluminiumverbindungen, sondern auf die bekannte Umlagerung der Alkinyl-substituierten Zirconocen-Komplexe zurückzuführen ist. ${ }^{[31,32,33]}$ Da von dem thermodynamisch stabileren Umlagerungsprodukt keine Reaktion zu einem Zirconocen-AluminiumVinyliden zu erwarten ist, wurde dieser Weg nicht weiter beschritten.
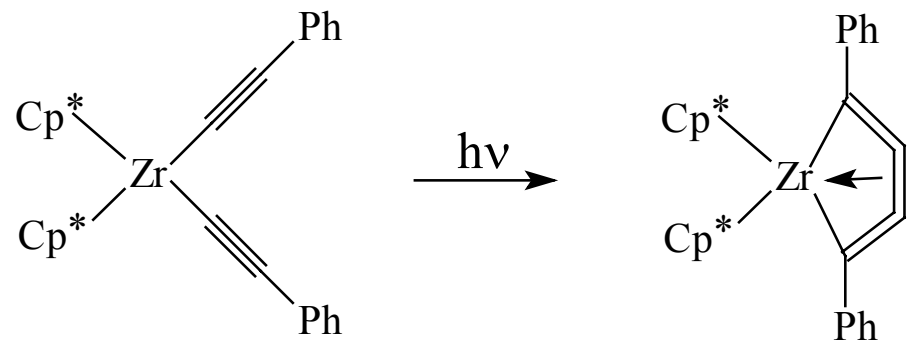

Schema 17: Umlagerung von $\mathrm{Cp}_{2}{ }_{2} \mathrm{Zr}(\mathrm{C} \equiv \mathrm{CPh})_{2}$. 


\subsection{Darstellung einer neuen Klasse von Aluminium-Verbindungen des Typs $\mathrm{M}\left[\mathrm{RAI}(\mathrm{C} \equiv \mathrm{CR})_{3}\right]$ mit drei terminalen Ethinyl-Gruppen und deren Interaktion mit Alkalimetall- Kationen}

\subsubsection{Einleitung}

Da sich die Darstellung der gewünschten Titanocen-Aluminium-Vinyliden-Derivate über Alkinyl-substituierte Metallocene als nicht erfolgreich erwiesen hatte, wurde ein anderer Weg eingeschlagen. Als Reaktionspartner von Dimethyl-Zirconocen sollten Ethinyl-Verbindungen der dreizehnten Gruppe des Periodensystems eingesetzt werden (vgl. Schema 10).

Als Dimethyl-Metallocen wurde das reaktive $\mathrm{Cp}_{2} \mathrm{ZrMe}_{2}$ eingesetzt, da hiermit bereits die Bildung einer $\mathrm{Zr}-\mathrm{C}_{\text {Alkinyl-Al-Bindung gelungen war (vgl. Schema 11). }}{ }^{[11,12]}$ Der Vorteil von $\mathrm{Cp}_{2} \mathrm{ZrMe}_{2}$ gegenüber $\mathrm{Cp}_{2} \mathrm{TiMe}_{2}$ ist, dass dieses kinetisch stabil ist (Das Titanocen-Derivat ist instabil, u.a. lichtempfindlich) ${ }^{[34,35]}$ und im Gegensatz zu $\mathrm{Cp}_{2} \mathrm{TiMe}_{2}$ eine Reduktion zu einer Metallocen(III)-Verbindung nicht zu befürchten ist (vgl. Kapitel 2.2.2.). ${ }^{[31]}$

Um zu den gewünschten Zielprodukten zu kommen, müssen die Zentralatome der dreizehnten Gruppe (Al, Ga, In) jedoch mindestens zwei Ethinylgruppen gebunden haben. In Frage kamen Verbindungen des Typs $\mathrm{R}_{\mathrm{n}} \mathrm{M}\left(\mathrm{C} \equiv \mathrm{C}-\mathrm{R}^{\prime}\right)_{3-\mathrm{n}}\left(\mathrm{n}=0,1 ; \mathrm{R}=\mathrm{z} \cdot \mathrm{B}\right.$. (Dipp) ${ }_{2} \mathrm{NacNac} ; \mathrm{R}^{\prime}=\mathrm{z} \cdot \mathrm{B} . \mathrm{Ph}$; $\mathrm{M}=\mathrm{Al}, \mathrm{Ga}, \mathrm{In})$ und neue, in dieser Arbeit vorzustellende Verbindungen des Typs $\mathrm{A}\left[\mathrm{RM}(\mathrm{C} \equiv \mathrm{C}-\mathrm{R})_{3}\right](\mathrm{M}=\mathrm{Al}, \mathrm{Ga}, \mathrm{In} ; \mathrm{R}=\mathrm{Rest} ; \mathrm{A}=$ Alkalimetall $)$.

Die Zahl der bekannten Verbindungen der o.g. Klasse war entweder sehr gering oder unbekannt. Ziel war es nun, die kaum entwickelte Ethinyl-Chemie der 13. Gruppe des Periodensystems voranzutreiben.

Am meisten wurde bisher auf dem Gebiet der Aluminium-Ethinyl-Verbindungen gearbeitet. So sind aus der Literatur ${ }^{[36]}$ fünf verschiedene Wege zur Darstellung von Aluminium-EthinylVerbindungen bekannt:

1.) Durch die Reaktion von $\left(\mathrm{AlMe}_{3}\right)_{2}$ mit Phenylethin ${ }^{[37]}$ $1 / 2\left(\mathrm{AlMe}_{3}\right)_{2}+\mathrm{H}-\mathrm{C} \equiv \mathrm{C}-\mathrm{Ph} \rightarrow \mathrm{Me}_{2} \mathrm{Al}-\mathrm{C} \equiv \mathrm{C}-\mathrm{Ph}+\mathrm{CH}_{4}$

2.) Durch Umsetzung von Dialkinyl-Quecksilber(II) mit Aluminium ${ }^{[36]}$ $3 \mathrm{Hg}-(\mathrm{C} \equiv \mathrm{C}-\mathrm{R})_{2}+2 \mathrm{Al} \rightarrow \mathrm{Al}-(\mathrm{C} \equiv \mathrm{C}-\mathrm{R})_{3} \quad(\mathrm{R}=$ org. Rest $)$

3.) Durch Reaktion von Aluminiumhydriden mit Dialkinyl-Quecksilber(II) ${ }^{[38]}$ $2 \mathrm{AlH}_{3} \cdot \mathrm{NEt}_{3}+3 \mathrm{Hg}-(\mathrm{C} \equiv \mathrm{C}-\mathrm{R})_{2} \rightarrow 2 \mathrm{Al}-(\mathrm{C} \equiv \mathrm{C}-\mathrm{R})_{3} \cdot \mathrm{NEt}_{3}+3 \mathrm{Hg}+3 \mathrm{H}_{2}$ $\left(\mathrm{R}=\mathrm{C}_{3} \mathrm{H}_{7}\right)$ 
4.) Die Reaktion eines Aluminiumhydrids mit einem Ethin-Derivat ${ }^{[39]}$

$$
\mathrm{R}_{2} \mathrm{AlH}+\mathrm{H}-\mathrm{C} \equiv \mathrm{C}-\mathrm{R} \rightarrow \mathrm{R}_{2} \mathrm{Al}-\mathrm{C} \equiv \mathrm{C}-\mathrm{R}+\mathrm{H}_{2} \quad(\mathrm{R}=\text { org. Rest })
$$

5.) Die Reaktion einer Aluminium-Halogenid-Verbindung mit einem Alkali-

Organoethinyl-Salz

$$
\mathrm{R}_{2} \mathrm{AlX}+\mathrm{M}-\mathrm{C} \equiv \mathrm{C}-\mathrm{R} \rightarrow \mathrm{R}_{2} \mathrm{Al}-\mathrm{C} \equiv \mathrm{C}-\mathrm{R}+\mathrm{MX} \quad(\mathrm{R}=\text { org. } \mathrm{Rest})
$$

Es gibt nur wenige strukturell charakterisierte Verbindungen mit mehr als einer terminalen Ethinylgruppe am Aluminium. ${ }^{[11,12,13,14]}$ Aus einer Veröffentlichung unseres Arbeitskreises $^{[14]}$ geht hervor, dass Aluminium-Ethinyl-Verbindungen entweder ölige Produkte ergeben oder dass sie schlecht in organischen Lösungsmitteln löslich sind. Daher wurde ein sterisch anspruchsvoller Ligand benutzt, dessen Derivate normalerweise gut kristallisieren und der einfach zugänglich ist $\left(2,6-i \mathrm{Pr}_{2} \mathrm{C}_{6} \mathrm{H}_{3} \mathrm{~N}\left(\mathrm{SiMe}_{3}\right)\right)$. Für die einfache Darstellung von Aluminium-Ethinyl-Verbindungen mit diesem Liganden boten sich zwei Möglichkeiten an:

a.) Aus dem entsprechenden Aluminiumhydrid und b.) aus dem entsprechenden Aluminiumhalogenid.

\subsubsection{Darstellung von $\left[2,6-i \mathrm{Pr}_{2} \mathrm{C}_{6} \mathrm{H}_{3} \mathrm{~N}\left(\mathrm{SiMe}_{3}\right) \mathrm{AlCl}_{2}\right]_{2}$}

Für die Darstellung neuer Aluminium-Diethinyl-Verbindungen mit 2,6-i $\mathrm{Pr}_{2} \mathrm{C}_{6} \mathrm{H}_{3} \mathrm{~N}\left(\mathrm{SiMe}_{3}\right)$ als Liganden wurde zunächst die bis dahin unbekannte Aluminiumhalogenid-Verbindung $\left[2,6-i \mathrm{Pr}_{2} \mathrm{C}_{6} \mathrm{H}_{3} \mathrm{~N}\left(\mathrm{SiMe}_{3}\right) \mathrm{AlCl}_{2}\right]_{2}$ (3) synthetisiert:

$\mathrm{Zu}$ einer Lösung von [2,6-i $\left.\mathrm{Pr}_{2} \mathrm{C}_{6} \mathrm{H}_{3} \mathrm{~N}\left(\mathrm{SiMe}_{3}\right) \mathrm{AlMe}_{2}\right]_{2}$ in Toluol wurden bei $-78{ }^{\circ} \mathrm{C}$ vier Äquivalente Trimethylzinnchlorid gegeben. Die Reaktionsmischung wurde bei Raumtemperatur über Nacht gerührt. Die leichtflüchtigen Bestandteile wurden im Vakuum entfernt und das Rohprodukt in Pentan aufgeschlämmt. Nach Filtration blieb 3 als farbloser Feststoff zurück.<smiles>[R][Al]([R])(C)N(C)C</smiles>

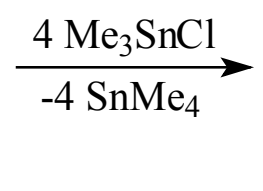<smiles>CC(C)c1cccc(C(C)C)c1N(S)[Al](Cl)(Cl)(Cl)(Cl)[Al](C)(Cl)Cl</smiles>

3

Schema 18: Darstellung von [2,6-i $\left.\mathrm{Pr}_{2} \mathrm{C}_{6} \mathrm{H}_{3} \mathrm{~N}\left(\mathrm{SiMe}_{3}\right) \mathrm{AlCl}_{2}\right]_{2}$ (3) $\left(\mathrm{R}=2,6-i \mathrm{Pr}_{2} \mathrm{C}_{6} \mathrm{H}_{3} \mathrm{~N}\left(\mathrm{SiMe}_{3}\right)\right)$. 
Das ${ }^{1}$ H-NMR-Spektrum von 3 zeigt ein Multiplett für die Arylprotonen, ein Septett für die zwei Protonen der beiden $i$-Propylgruppen, zwei Dubletts für die Methylprotonen der $i$-Propylgruppen und ein Singulett der Protonen der TMS-Gruppe. Im Massenspektrum findet man bei 345 den Molpeak (m/e) des Monomers. Die Ergebnisse der Elementaranalyse entsprechen den für $\mathbf{3}$ berechneten Werten.

Um endgültigen Aufschluss über den molekularen Aufbau von $3 \mathrm{zu}$ erhalten, wurde eine Röntgenstrukturanalyse durchgeführt. Hierfür geeignete Kristalle wurden durch langsames Entfernen des Lösungsmittels (Toluol) bei Raumtemperatur erhalten. Die EinkristallRöntgenkristallstruktur belegt, dass die Verbindung als halogenidverbrücktes, solvatfreies Dimer vorliegt.
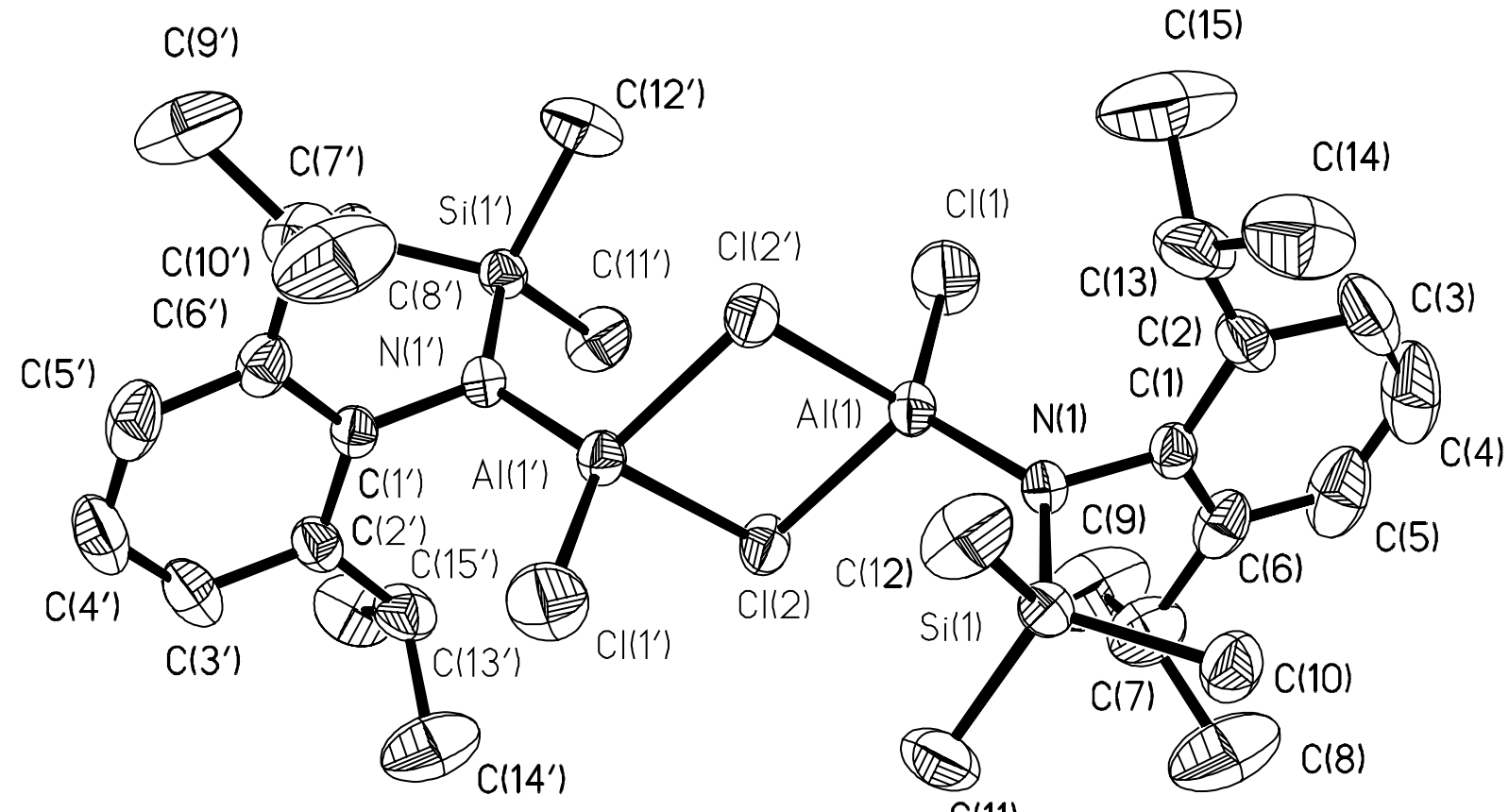

C(11)

Abb. 2: Struktur von 3 im Kristall.

Tabelle 2: Ausgewählte Bindungslängen $(\AA)$ und Winkel $\left(^{\circ}\right)$ für 3.

$\begin{array}{llll}\mathrm{Al}\left(1^{\prime}\right)-\mathrm{N}\left(1^{\prime}\right) & 1.777(2) & \mathrm{Al}(1)-\mathrm{Cl}(1) & 2.0844(12) \\ \mathrm{Al}\left(1^{\prime}\right)-\mathrm{Cl}\left(1^{\prime}\right) & 2.0667(12) & \mathrm{Al}(1)-\mathrm{Cl}(2) & 2.2729(11) \\ \mathrm{Al}\left(1^{\prime}\right)-\mathrm{Cl}\left(2^{\prime}\right) & 2.2813(11) & \mathrm{Al}(1)-\mathrm{Cl}\left(2^{\prime}\right) & 2.2773(11) \\ \mathrm{Al}\left(1^{\prime}\right)-\mathrm{Cl}(2) & 2.2855(11) & \mathrm{Cl}\left(1^{\prime}\right)-\mathrm{Al}\left(1^{\prime}\right)-\mathrm{Cl}\left(2^{\prime}\right) & 106.48(5) \\ \mathrm{N}(1)-\mathrm{Al}(1) & 1.774(2) & \mathrm{Cl}\left(1^{\prime}\right)-\mathrm{Al}\left(1^{\prime}\right)-\mathrm{Cl}(2) & 106.58(5)\end{array}$




$\begin{array}{llll}\mathrm{Cl}\left(2^{\prime}\right)-\mathrm{Al}\left(1^{\prime}\right)-\mathrm{Cl}(2) & 89.38(4) & \mathrm{Al}(1)-\mathrm{Cl}\left(2^{\prime}\right)-\mathrm{Al}\left(1^{\prime}\right) & 90.41(4) \\ \mathrm{Cl}(1)-\mathrm{Al}(1)-\mathrm{Cl}(2) & 106.16(5) & \mathrm{N}\left(1^{\prime}\right)-\operatorname{Al}\left(1^{\prime}\right)-\mathrm{Cl}\left(1^{\prime}\right) & 121.05(8) \\ \mathrm{Cl}(1)-\mathrm{Al}(1)-\mathrm{Cl}\left(2^{\prime}\right) & 105.77(5) & \mathrm{N}\left(1^{\prime}\right)-\operatorname{Al}\left(1^{\prime}\right)-\mathrm{Cl}\left(2^{\prime}\right) & 114.75(8) \\ \mathrm{Cl}(2)-\mathrm{Al}(1)-\mathrm{Cl}\left(2^{\prime}\right) & 89.79(4) & \mathrm{Cl}\left(1^{\prime}\right)-\mathrm{Al}\left(1^{\prime}\right)-\mathrm{Cl}\left(2^{\prime}\right) & 106.48(5) \\ \mathrm{Al}(1)-\mathrm{Cl}(2)-\operatorname{Al}\left(1^{\prime}\right) & 90.42(4) & \mathrm{N}\left(1^{\prime}\right)-\operatorname{Al}\left(1^{\prime}\right)-\mathrm{Cl}(2) & 113.83(8)\end{array}$

Die Bindungslängen, die in dem viergliedrigen $\mathrm{Al}_{2} \mathrm{Cl}_{2}$ - Ring auftreten, (2.273-2.286 $\AA$ ) korrespondieren mit denen bekannter Verbindungen (vgl. Tab. 2)

Tabelle 2: Bindungslängen vergleichbarer Verbindungen:

\begin{tabular}{|l|l|l|}
\hline & Al-Cl (terminal) $(\AA)$ & $\mathrm{Al}-\mathrm{Cl}($ verbrückt) $(\AA)$ \\
\hline$\left[2,6-\mathrm{Mes}_{2} \mathrm{C}_{6} \mathrm{H}_{3} \mathrm{AlCl}_{2}\right]_{2}{ }^{[40]}$ & $2.104-2.118$ & $2.171-2.191$ \\
\hline$\left[\mathrm{AlCl}_{2} \mathrm{Me}\right]_{2}{ }^{[41]}$ & 2.05 & $2.24-2.26$ \\
\hline$\left[\mathrm{Cl}_{2} \mathrm{Al}-\mathrm{CH}\left(\mathrm{SiMe}_{3}\right)_{2}\right]_{2}{ }^{[42]}$ & 2.068 & $2.278-2.293$ \\
\hline
\end{tabular}

Ein Chloratom ist terminal an das Aluminium gebunden. Diese Bindung (2.066$2.084 \AA$ ) ist kürzer und liegt ebenfalls im Bereich bekannter Substanzen (vgl. Tab. 2). Die tetraedrische Koordination am Aluminium ist stark verzerrt. So findet man den $\mathrm{Cl}\left(2^{\prime}\right)-\mathrm{Al}\left(1^{\prime}\right)$ $\mathrm{Cl}(2)-W i n k e l$ bei $89.38^{\circ}$, während der $\mathrm{N}\left(1^{\prime}\right)-\mathrm{Al}\left(1^{\prime}\right)-\mathrm{Cl}\left(1^{\prime}\right)-$ Winkel $121.05^{\circ}$ beträgt.

\subsubsection{Darstellung von $\left[\mathrm{K} \cdot \mathrm{THF}\left(2,6-i \mathrm{Pr}_{2} \mathrm{C}_{6} \mathrm{H}_{3} \mathrm{~N}\left(\mathrm{SiMe}_{3}\right) \mathrm{Al}(\mathrm{C} \equiv \mathrm{CPh})_{3}\right)_{2}(4)\right.$ und von $\left[\mathrm{Na} \cdot \operatorname{THF}\left(2,6-i \mathrm{Pr}_{2} \mathrm{C}_{6} \mathrm{H}_{3} \mathrm{~N}\left(\mathrm{SiMe}_{3}\right) \mathrm{Al}(\mathrm{C} \equiv \mathrm{CPh})_{3}\right)\right]_{2}(5)$}

Bei der Umsetzung von Verbindung 3 mit drei Äquivalenten eines Alkalimetall-EthinylSalzes wurden die ersten Aluminium-Verbindungen des Typs $M\left[R A l(C \equiv C R)_{3}\right](M=K, N a$, $\mathrm{Li} ; \mathrm{R}=\mathrm{Ph}$ ) erhalten, bei denen drei terminale Ethinyl-Liganden an das Aluminiumzentrum gebunden sind. Ein Alkalimetall-Kation fungiert als Gegenion zu dem Organo-AluminiumTriethinyl-Anion. 


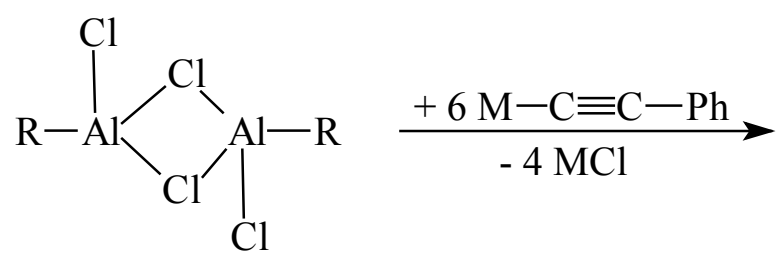

3

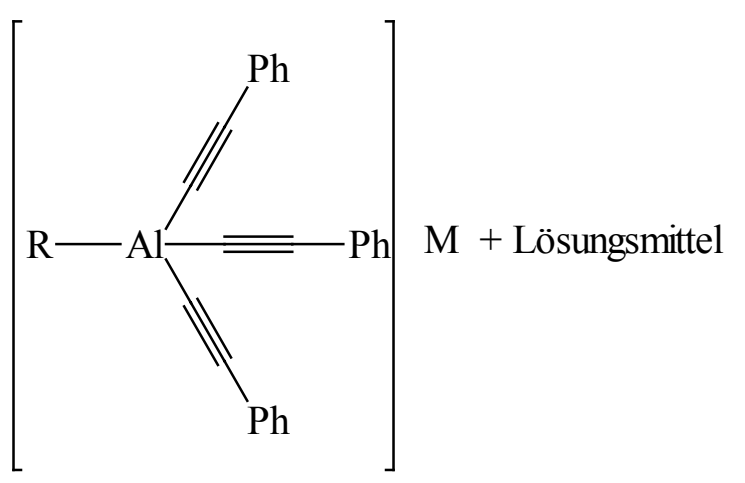

$\mathrm{M}=\mathrm{K}(\mathbf{4}) ; \mathrm{Na}(\mathbf{5}) ; \mathrm{Li}(\mathbf{6})$

$\mathrm{R}=2,6-i \mathrm{Pr}_{2} \mathrm{C}_{6} \mathrm{H}_{3} \mathrm{~N}\left(\mathrm{SiMe}_{3}\right)$

Schema 19: Darstellung von 4, 5 und 6.

Verbindungen 4 und 5 wurden bei der Umsetzung von $\left[\mathrm{RAlCl}_{2}\right]_{2}(\mathbf{3})$ mit sechs Äquivalenten Kalium- bzw. Natrium-Phenylethinyl in THF bei erhöhter Temperatur $\left(50{ }^{\circ} \mathrm{C}\right)$ erhalten. Die Aryl-Protonen der Verbindung 4 erzeugen im ${ }^{1} \mathrm{H}-\mathrm{NMR}$ ein Multiplett bei $\delta$ 7.3-6.6, während die Aryl-Protonen von 5 ein Multiplett bei $\delta$ 7.5-6.7 ergeben. Die übrigen Resonanzen können dem 2,6-i $\operatorname{Pr}_{2} \mathrm{C}_{6} \mathrm{H}_{3} \mathrm{~N}\left(\mathrm{SiMe}_{3}\right)$-Rest zugeordnet werden. Im ${ }^{13} \mathrm{C}-\mathrm{NMR}-$ Spektrum sieht man aufgrund des Quadrupol-Effekts das auffällig breite Signal der ${ }^{13} \mathrm{C}_{\text {Alkinyl-Atome, die an das }}$ Aluminiumatom gebunden sind, bei $\delta 113.5$ (4) bzw. $\delta 112.5$ (5). Im Massenspektrum von 4 wird das Anion $\left(\mathrm{RAl}(\mathrm{C} \equiv \mathrm{CPh})_{3}\right)^{-}$bei 578 detektiert.

Geeignete Einkristalle für eine Röntgenstrukturanalyse konnten durch langsames Eindiffundieren von Hexan in eine gesättigte THF-Lösung von Verbindung 4 bzw. 5 gewonnen werden.

Der molekulare Aufbau der Verbindungen 4 und 5 konnte per Röntgenstrukturanalyse untersucht werden und wird in Abb. 3 wiedergegeben. 


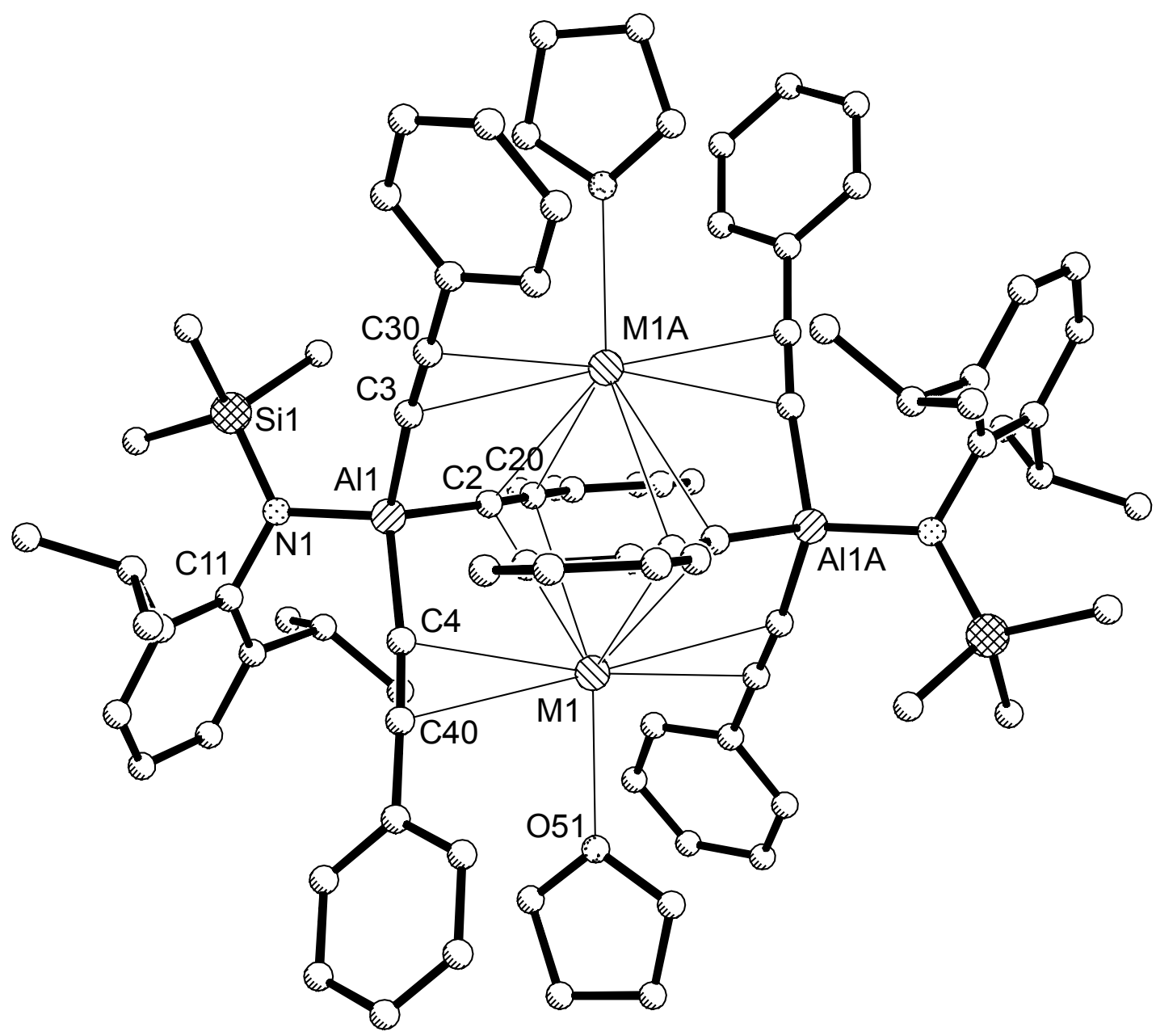

Abb. 3. : Kugelmodell der isomorphen Verbindungen 4 und $5(\mathrm{M}=\mathrm{K}(\mathbf{4}) ; \mathrm{Na}(5))$.

Ausgewählte Bindungslängen ( $(̊)$ und Winkel ( $\left.{ }^{\circ}\right)$ für 4.

$\begin{array}{lllc}\mathrm{K}(1)-\mathrm{C}(4) & 2.969(2) & \mathrm{C}(3 \mathrm{~A})-\mathrm{K}(1)-\mathrm{C}(30 \mathrm{~A}) & 22.68(6) \\ \mathrm{K}(1)-\mathrm{C}(3 \mathrm{~A}) & 3.035(2) & \mathrm{C}(4)-\mathrm{K}(1)-\mathrm{C}(40) & 22.22(6) \\ \mathrm{K}(1)-\mathrm{C}(2 \mathrm{~A}) & 3.063(2) & \mathrm{C}(2 \mathrm{~A})-\mathrm{K}(1)-\mathrm{C}(20 \mathrm{~A}) & 20.78(6) \\ \mathrm{K}(1)-\mathrm{C}(30 \mathrm{~A}) & 3.089(2) & \mathrm{C}(4)-\mathrm{Al}(1)-\mathrm{C}(3) & 110.45(11) \\ \mathrm{K}(1)-\mathrm{C}(2) & 3.097(2) & \mathrm{C}(4)-\mathrm{Al}(1)-\mathrm{C}(2) & 107.27(10) \\ \mathrm{K}(1)-\mathrm{C}(40) & 3.191(3) & \mathrm{C}(3)-\mathrm{Al}(1)-\mathrm{C}(2) & 104.68(10) \\ \mathrm{K}(1)-\mathrm{C}(20 \mathrm{~A}) & 3.399(3) & \mathrm{C}(20)-\mathrm{C}(2)-\mathrm{Al}(1) & 165.7(2) \\ \mathrm{K}(1)-\mathrm{C}(20) & 3.473(3) & \mathrm{C}(30)-\mathrm{C}(3)-\mathrm{Al}(1) & 175.0(2) \\ \mathrm{Al}(1)-\mathrm{C}(4) & 1.962(3) & \mathrm{C}(40)-\mathrm{C}(4)-\mathrm{Al}(1) & 173.6(2) \\ \mathrm{Al}(1)-\mathrm{C}(3) & 1.963(3) & \mathrm{N}(1)-\mathrm{Al}(1)-\mathrm{C}(4) & 108.62(9) \\ \mathrm{Al}(1)-\mathrm{C}(2) & 1.984(3) & \mathrm{N}(1)-\mathrm{Al}(1)-\mathrm{C}(3) & 114.13(10) \\ \mathrm{Al}(1)-\mathrm{N}(1) & 1.836(2) & & \end{array}$




\section{Ausgewählte Bindungslängen $(\AA)$ und Winkel $\left({ }^{\circ}\right)$ für 5.}

$\begin{array}{lllc}\mathrm{Na}(1)-\mathrm{C}(4) & 2.651(4) & \mathrm{C}(3) \mathrm{A}-\mathrm{Na}(1)-\mathrm{C}(30) \mathrm{A} & 24.76(10) \\ \mathrm{Na}(1)-\mathrm{C}(3) \mathrm{A} & 2.707(4) & \mathrm{C}(4)-\mathrm{Na}(1)-\mathrm{C}(40) & 23.88(10) \\ \mathrm{Na}(1)-\mathrm{C}(2) \mathrm{A} & 2.772(4) & \mathrm{C}(2)-\mathrm{Na}(1)-\mathrm{C}(20) & 20.6(1) \\ \mathrm{Na}(1)-\mathrm{C}(20) \mathrm{A} & 3.221(4) & \mathrm{C}(4)-\mathrm{Al}(1)-\mathrm{C}(3) & 110.94(16) \\ \mathrm{Na}(1)-\mathrm{C}(20) & 3.358(4) & \mathrm{C}(4)-\mathrm{Al}(1)-\mathrm{C}(2) & 106.13(16) \\ \mathrm{Na}(1)-\mathrm{C}(2) & 2.876(4) & \mathrm{C}(3)-\mathrm{Al}(1)-\mathrm{C}(2) & 103.72(15) \\ \mathrm{Na}(1)-\mathrm{C}(30) \mathrm{A} & 2.888(4) & \mathrm{C}(20)-\mathrm{C}(2)-\mathrm{Al}(1) & 164.2(3) \\ \mathrm{Na}(1)-\mathrm{C}(40) & 2.968(4) & \mathrm{C}(30)-\mathrm{C}(3)-\mathrm{Al}(1) & 176.7(3) \\ \mathrm{Al}(1)-\mathrm{C}(4) & 1.960(4) & \mathrm{C}(30)-\mathrm{C}(3)-\mathrm{Na}(1) \mathrm{A} & 86.0(2) \\ \mathrm{Al}(1)-\mathrm{C}(3) & 1.965(4) & \mathrm{N}(1)-\mathrm{Al}(1)-\mathrm{C}(4) & 108.93(14) \\ \mathrm{Al}(1)-\mathrm{C}(2) & 1.988(4) & \mathrm{N}(1)-\mathrm{Al}(1)-\mathrm{C}(3) & 113.49(15) \\ \mathrm{Al}(1)-\mathrm{N}(1) & 1.834(3) & & \end{array}$

Das Besondere an diesen Verbindungen ist die Interaktion des Alkalimetall-Kations mit den Phenylethinyl-Liganden. Diese Kation- $\pi$-Wechselwirkungen spielen eine herausragende Rolle unter den vielen nicht kovalenten Bindungen und sind daher Bestandteil einer Vielzahl von Veröffentlichungen. ${ }^{[43,44,45,46,47]}$

Es sind zwar drei Kalium-Verbindungen dieses Bindungstyps strukturell charakterisiert worden, ${ }^{[48,49,50]}$ dennoch ist $\mathbf{4}$ das erste Beispiel einer Verbindung, bei der Kalium ausschließlich zu vier Alkinyl-Liganden und einem THF- Molekül koordiniert ist.

Im Falle von Natrium gibt es drei bekannte Alkinyl-Verbindungen mit dieser Bindungsart, die strukturell charakterisiert worden sind. ${ }^{[51,52,53]}$ Unseres Wissens sind keine AluminiumAlkinyl-Verbindungen mit einer Kation- $\pi$-Wechselwirkung bekannt.

Die Einkristall-Röntgenstrukturanalyse von 4 und $\mathbf{5}$ gibt Aufschluss über die räumliche Anordnung der Kalium- bzw. Natrium-Ionen, die jeweils von vier Ethinyl-Einheiten $\pi$ koordiniert werden. Die fünfte Koordinationsstelle nimmt ein THF- Molekül ein. Verbindungen 4 und 5 besitzen ein Inversionszentrum. Die $\left[2,6-i \mathrm{Pr}_{2} \mathrm{C}_{6} \mathrm{H}_{3} \mathrm{~N}\left(\mathrm{SiMe}_{3}\right) \mathrm{Al}(\mathrm{C} \equiv \mathrm{CPh})_{3}\right]^{-}$-Käfige werden durch die zwei dazwischen liegenden Kalium- bzw. Natrium-Ionen zusammengehalten, die mit den $\pi$-Elektronen der Ethinylgruppen wechselwirken. Bei leicht verzerrter tetraedrischer Koordination des Aluminiumatoms werden drei Koordinationsstellen von Phenylethinyl-Gruppen und eine mit dem 2,6-i $\mathrm{Pr}_{2} \mathrm{C}_{6} \mathrm{H}_{3} \mathrm{~N}\left(\mathrm{SiMe}_{3}\right)$-Substituenten besetzt. Die Al-C-Bindungslänge zwischen dem 
Aluminium- und dem Ethinyl-Kohlenstoffatom (1.960-1.988 Å) findet man im Erwartungsbereich. ${ }^{[13,14]}$ Die Winkel zwischen dem Aluminiumatom und den zwei EthinylKohlenstoffatomen liegen zwischen $164.2^{\circ}$ und $176.7^{\circ}$. Die starke Abweichung von der Linearität der $\mathrm{M}-\mathrm{C} \equiv \mathrm{C}$-Einheit, die von zwei Kalium- bzw. Natrium-Ionen koordiniert wird $\left(\mathrm{Al}(1)-\mathrm{C}(2)-\mathrm{C}(20) 165.7^{\circ}\right.$ (4); ( $\left.\mathrm{Al}(1)-\mathrm{C}(2)-\mathrm{C}(20) 164.2^{\circ}(5)\right)$, ist das fünfte Beispiel für ein Hauptgruppenmetall und das zweite für Aluminium. ${ }^{[14,51]}$ Die Ursache ist hier wohl in der geringen Energiedifferenz zwischen linearen und gewinkelten $\mathrm{M}-\mathrm{C} \equiv \mathrm{C}$ Einheiten zu suchen.

Der Abstand des Kalium- bzw. Natrium-Kations zu den Ethinyl-Kohlenstoffatomen liegt zwischen 2.969 und $3.473 \AA$ (4) und zwischen 2.651 und $3.358 \AA$ (5) und ist damit deutlich größer als der Abstand von bekannten Verbindungen dieses Typus $\left(\mathrm{K}-\mathrm{C}_{\text {alkinyl }}\right.$ 2.868-3.197 $\AA$; Na-C $\mathrm{C}_{\text {alkinyl }}$ 2.533-2.891 $\AA$ ). ${ }^{[48-53]}$ Es ist offensichtlich, dass der Unterschied der Bindungsabstände bei 5 größer ist $(0.707 \AA)$ als bei dem korrespondierenden in 4 (0.504 $\AA$ ).

Da es sich bei $\mathbf{4}$ und $\mathbf{5}$ jedoch um die ersten Verbindungen mit Aluminium als Zentralatom

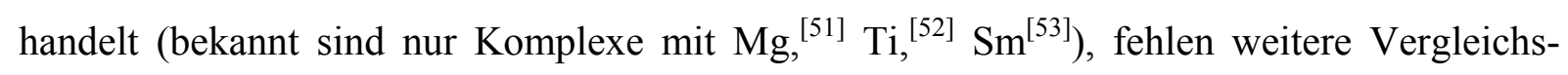
möglichkeiten. Die Winkel, die am Kalium- bzw. Natriumatom mit den Ethinyl-Kohlenstoffatomen gebildet werden, liegen zwischen $20.23-22.68^{\circ}$ (4) und 20.6-24.76 . Vergleichbare Werte findet man bei anderen Alkalimetall-Verbindungen. ${ }^{[48,51,52,53]}$
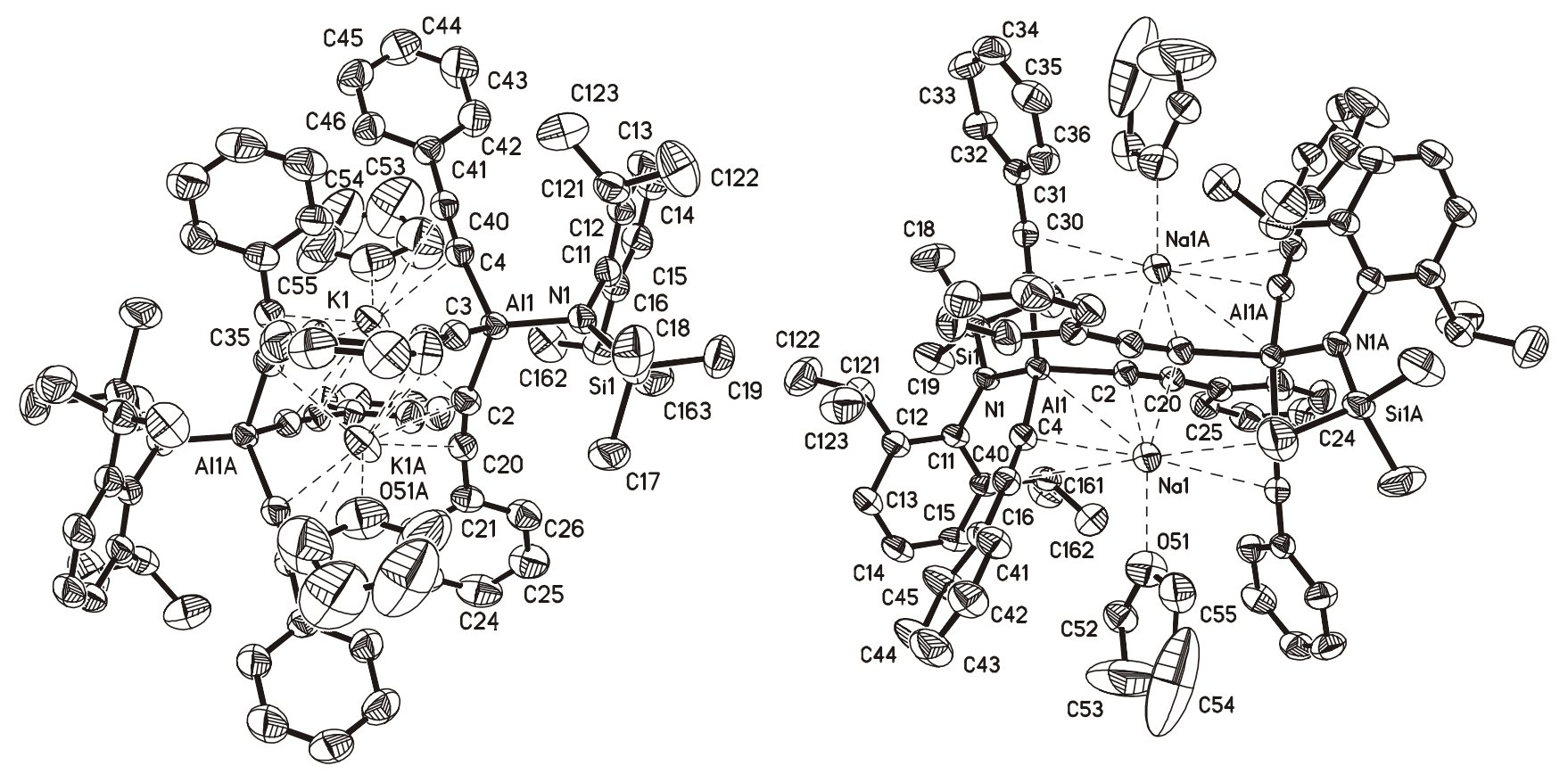

Abb. 4: Struktur von 4 und 5 im Kristall. 


\subsubsection{Darstellung von}

\section{$\left[\mathrm{Li} \cdot \operatorname{Dioxan}\left(2,6-i \mathrm{Pr}_{2} \mathrm{C}_{6} \mathrm{H}_{3} \mathrm{~N}\left(\mathrm{SiMe}_{3}\right) \mathrm{Al}(\mathrm{C} \equiv \mathrm{CPh})_{3}\right)\right]_{2} \cdot 2$ Dioxan (6)}

$\mathrm{Zu}$ Verbindung 3 in Dietylether wurden sechs Äquivalente Lithium-Phenylethinyl (vgl. Schema 19) gegeben. Das Gemisch wurde bei Raumtemperatur über Nacht gerührt und nachdem der Ether im Vakuum entfernt worden ist, das entstandene ölige Produkt mit Dioxan versetzt. Es fiel ein weißer Niederschlag aus. Dieser wurde mit Hexan gewaschen und man erhielt 6 in guter Ausbeute (60\%).

Das ${ }^{1}$ H-NMR- Spektrum zeigt ein Multiplett für die Aryl-Protonen bei $\delta$ 7.4-6.8. Die übrigen Resonzen können dem 2,6-i $\mathrm{Pr}_{2} \mathrm{C}_{6} \mathrm{H}_{3} \mathrm{~N}\left(\mathrm{SiMe}_{3}\right)$-Substituenten zugeordnet werden. Im ${ }^{13} \mathrm{C}$ NMR-Spektrum ist das Signal der in $\alpha$-Position zum Aluminium gebundenen Kohlenstoffatome aufgrund des Quadrupol-Effekts sehr breit und liegt bei $\delta$ 109.0. Das ${ }^{7} \mathrm{Li}$ NMR zeigt eine Resonanz bei $\delta$ 0.2. Die bei der Elementaranalyse gefundenen Werte bestätigen die Zusammensetzung $\left(\mathrm{C}_{94} \mathrm{H}_{114} \mathrm{Al}_{2} \mathrm{Li}_{2} \mathrm{~N}_{2} \mathrm{O}_{8} \mathrm{Si}_{2}\right)$.

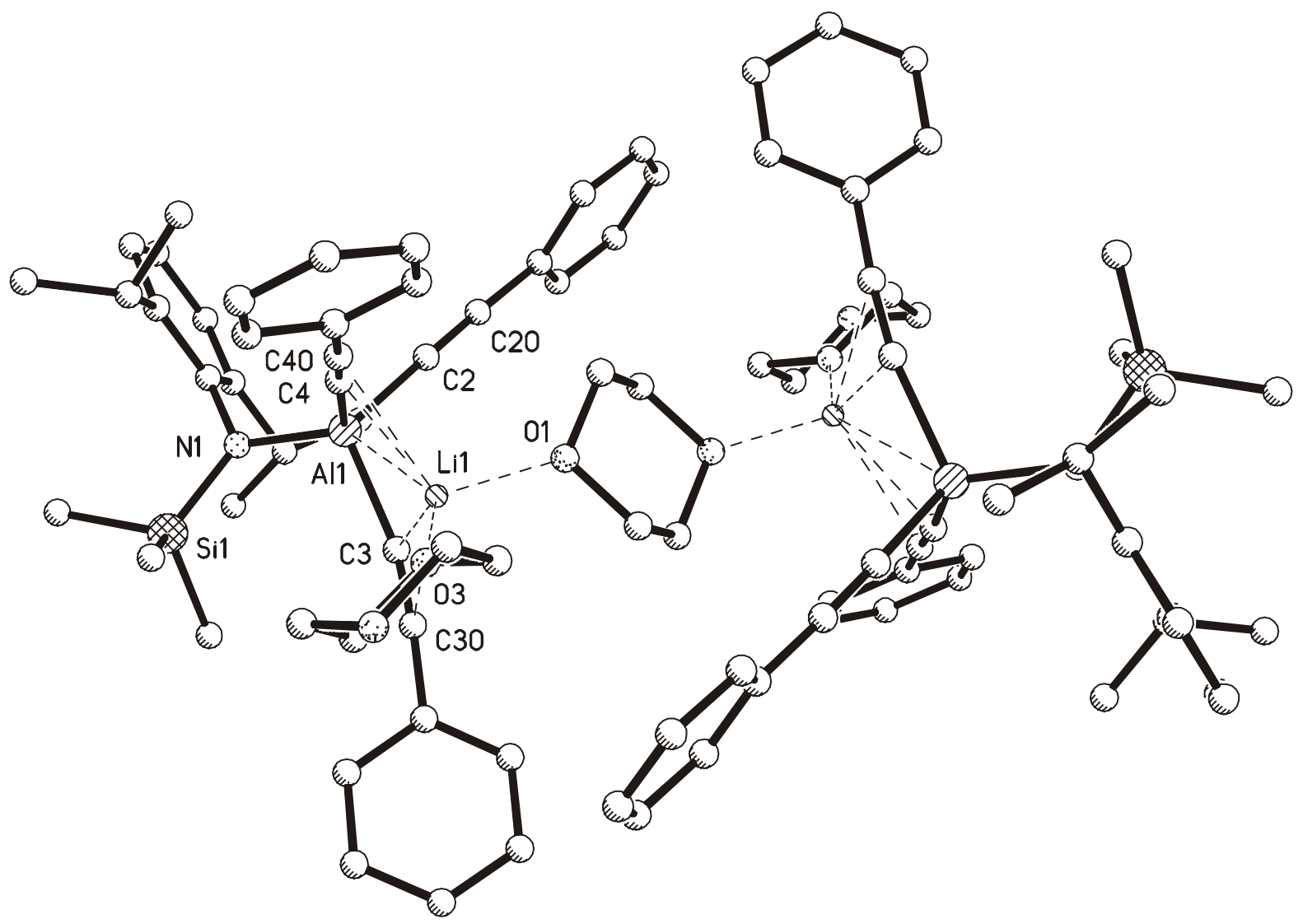

Abb. 5: Struktur von 6 im Kristall. Ein weiteres Dioxanmolekül ist nicht dargestellt. 
Ausgewählte Bindungslängen $(\AA ̊)$ und Winkel $\left(^{\circ}\right)$ für 6.

$\begin{array}{lccc}\mathrm{Al}(1)-\mathrm{C}(2) & 1.943(3) & \mathrm{C}(2)-\mathrm{Al}(1)-\mathrm{C}(3) & 113.01(12) \\ \mathrm{Al}(1)-\mathrm{C}(4) & 1.992(3) & \mathrm{C}(4)-\mathrm{Al}(1)-\mathrm{C}(3) & 97.09(12) \\ \mathrm{Al}(1)-\mathrm{C}(3) & 1.998(3) & \mathrm{C}(4)-\mathrm{Li}(1)-\mathrm{C}(40) & 29.21(10) \\ \mathrm{Li}(1)-\mathrm{C}(4) & 2.287(5) & \mathrm{C}(3)-\mathrm{Li}(1)-\mathrm{C}(30) & 27.76(10) \\ \mathrm{Li}(1)-\mathrm{C}(3) & 2.350(5) & \mathrm{C}(20)-\mathrm{C}(2)-\mathrm{Al}(1) & 175.6(2) \\ \mathrm{Li}(1)-\mathrm{C}(40) & 2.464(6) & \mathrm{C}(30)-\mathrm{C}(3)-\mathrm{Al}(1) & 166.6(2) \\ \mathrm{Li}(1)-\mathrm{C}(30) & 2.594(6) & \mathrm{C}(40)-\mathrm{C}(4)-\mathrm{Al}(1) & 173.8(2) \\ \mathrm{Al}(1)-\mathrm{N}(1) & 1.837(4) & \mathrm{N}(1)-\mathrm{Al}(1)-\mathrm{C}(2) & 114.72(15) \\ \mathrm{C}(2)-\mathrm{Al}(1)-\mathrm{C}(4) & 106.61(14) & \mathrm{N}(1)-\mathrm{Al}(1)-\mathrm{C}(4) & 114.61(13)\end{array}$

Im Gegensatz zu den Verbindungen 4 und 5 ist hier das Lithium-Ion an nur zwei Phenylethinyl-Liganden und an zwei Lösungsmittel-Moleküle gebunden. Es fungiert nicht als verbrückende Einheit der $\left[2,6-i \mathrm{Pr}_{2} \mathrm{C}_{6} \mathrm{H}_{3} \mathrm{~N}\left(\mathrm{SiMe}_{3}\right) \mathrm{Al}(\mathrm{C} \equiv \mathrm{CPh})_{3}\right]^{-}$-Gruppen. Diese Funktion übernimmt ein Dioxan-Molekül. Die Al-C-Bindungslängen (1.943-1.998 $\AA$ ) sind mit denen von 4 und 5 vergleichbar. Auch die Al-C $\equiv \mathrm{C}$-Bindungswinkel $\left(166.6-175.6^{\circ}\right)$ sind denen der Verbindung 4 und 5 ähnlich. Die Koordination der Liganden am Aluminiumatom ist hier erwartungsgemäß tetraedrisch verzerrt. Die Verzerrung nimmt in der Reihenfolge $\mathrm{Li}^{+}>\mathrm{Na}^{+}>$ $\mathrm{K}^{+}$ab. Dies hängt wohl mit den deutlich abnehmenden Bindungslängen $\left(\mathrm{Li}^{+}-\mathrm{C}_{\text {Alkinyl }}<\mathrm{Na}^{+}-\right.$ $\mathrm{C}_{\text {Alkinyl }}<\mathrm{K}^{+}-\mathrm{C}_{\text {Alkinyl }}$ ) vom Alkalimetall-Ion zu den Ethinyl-Kohlenstoffatomen zusammen.

\subsubsection{Diskussion der Verbindungen 4, 5 und 6 .}

Insgesamt wurde das Ergebnis der Strukturuntersuchungen von 4, 5 und 6 nicht erwartet, denn gemäß dem Pearsonkonzept harter und weicher Säuren (HSAB $)^{[54]}$ koordiniert zwar das harte Kalium- bzw. Natrium-Kation mit dem harten Sauerstoff des THF-Moleküls bzw. des Dioxan-Moleküls, aber vier weitere Koordinationen werden zu den weicheren $\pi$-Systemen der Alkinyl-Gruppen ausgebildet, so dass sich die Koordinationszahl fünf am Kalium- bzw. Natrium ausbildet. Darüber hinaus kann man sehen, dass auch im Überschuss des Lösungsmittels THF das Kalium- bzw. Natrium-Kation die "Tasche" mit den vier Alkinyl-Gruppen bevorzugt. Die gebildeten Produkte scheinen im Vergleich zu den vollständig solvatisierten Kationen wie $\left[\mathrm{K}(\mathrm{THF})_{6}\right]^{+}$energetisch bevorzugt zu sein. 


\subsection{Darstellung einer neuen Klasse von Verbindungen der 13. Gruppe des Periodensystems mit drei terminalen Trimethylsilylethinyl- Liganden des Typs $\left[\mathrm{RM}\left(\mathrm{C} \equiv \mathrm{CSiMe}_{3}\right)_{3}\right]^{-}$}

\subsubsection{Darstellung von}

\section{$\left[\mathrm{Li} \cdot 2\right.$ Dioxan $\left.\left(2,6-i \mathrm{Pr}_{2} \mathrm{C}_{6} \mathrm{H}_{3} \mathrm{~N}\left(\mathrm{SiMe}_{3}\right) \mathrm{Al}\left(\mathrm{C} \equiv \mathrm{CSiMe}_{3}\right)_{3}\right)\right] \cdot 0.75$ Dioxan (7)}

3 wurde in THF mit sechs Äquivalenten $\mathrm{LiC} \equiv \mathrm{CSiMe}_{3}$ versetzt und das Gemisch über Nacht bei Raumtemperatur gerührt. Dann wurde auf $55^{\circ} \mathrm{C}$ erwärmt und man erhielt die Verbindung [Li $\cdot 2$ Dioxan $\left.\left(2,6-i \mathrm{Pr}_{2} \mathrm{C}_{6} \mathrm{H}_{3} \mathrm{~N}\left(\mathrm{SiMe}_{3}\right) \mathrm{Al}\left(\mathrm{C} \equiv \mathrm{CSiMe}_{3}\right)_{3}\right)\right] \cdot 0.75$ Dioxan (7) als farblosen Feststoff. Trotz des sterisch anspruchsvollen Substituenten erhielt man eine AluminiumEthinyl-Verbindung mit drei terminalen Ethinyl-Liganden, die an das Aluminium gebunden sind, die vierte Koordinationsstelle wird von einem (2,6-i $\mathrm{Pr}_{2} \mathrm{C}_{6} \mathrm{H}_{3} \mathrm{~N}\left(\mathrm{SiMe}_{3}\right)$-Liganden besetzt. Bei der alternativen Darstellung von 7 wurde 1 in Toluol gelöst und ein Überschuss an $\mathrm{HC} \equiv \mathrm{CSSiMe}_{3}$ hinzu gegeben. Nach ein paar Tagen bildeten sich farblose Kristalle, welche nach Zugabe von Dioxan als 7 identifiziert werden konnten.

Das ${ }^{1} \mathrm{H}$-NMR-Spektrum zeigt die Resonanz der $\mathrm{N}_{-} \mathrm{SiMe}_{3}$-Gruppe bei $\delta 0.07$ und die der drei an Ethinyleinheiten gebundenen $\mathrm{SiMe}_{3}$-Gruppen bei $\delta-0.04 \mathrm{im}$ Intensitätsverhältnis 1:3. Anhand der Intensität der Protonen des Dioxans kann man erkennen, dass nach dem Trocknen im Vakuum nur noch zwei Dioxan-Moleküle koordiniert sind. Die ${ }^{7} \mathrm{Li}$-Ionen erzeugen im ${ }^{7} \mathrm{Li}$ NMR ein Signal bei $\delta-0.3$, während das ${ }^{29}$ Si-NMR-Spektrum zwei Resonanzen für die beiden unterschiedlich gebundenen $\mathrm{SiMe}_{3}$-Gruppen zeigt $(\delta-3.6 ;-25.3)$. Im ${ }^{13} \mathrm{C}-\mathrm{NMR}-$ Spektrum sind die Resonanzen der in $\alpha$-Position an das Aluminium gebundenen Kohlenstoffatome bei $\delta 109.0$ und die der in $\beta$-Position liegenden Kohlenstoffatome bei $\delta 95.3 \mathrm{zu}$ sehen. Das Erscheinungsbild der Signale der in $\alpha$-Position an das Aluminium gebundenen ${ }^{13} \mathrm{C}$-Kerne ist breit, da die benachbarten Aluminium-Kerne ein elektrisches KernQuadrupolmoment besitzen. ${ }^{[55]}$

Im Massenspektrum ist der molare Massen-Peak des Anions (566) zu beobachten. Die Elementaranalyse bestätigt die gefundene Zusammensetzung $\mathrm{C}_{38} \mathrm{H}_{69} \mathrm{AlLiNO}_{4} \mathrm{Si}_{4}$. 


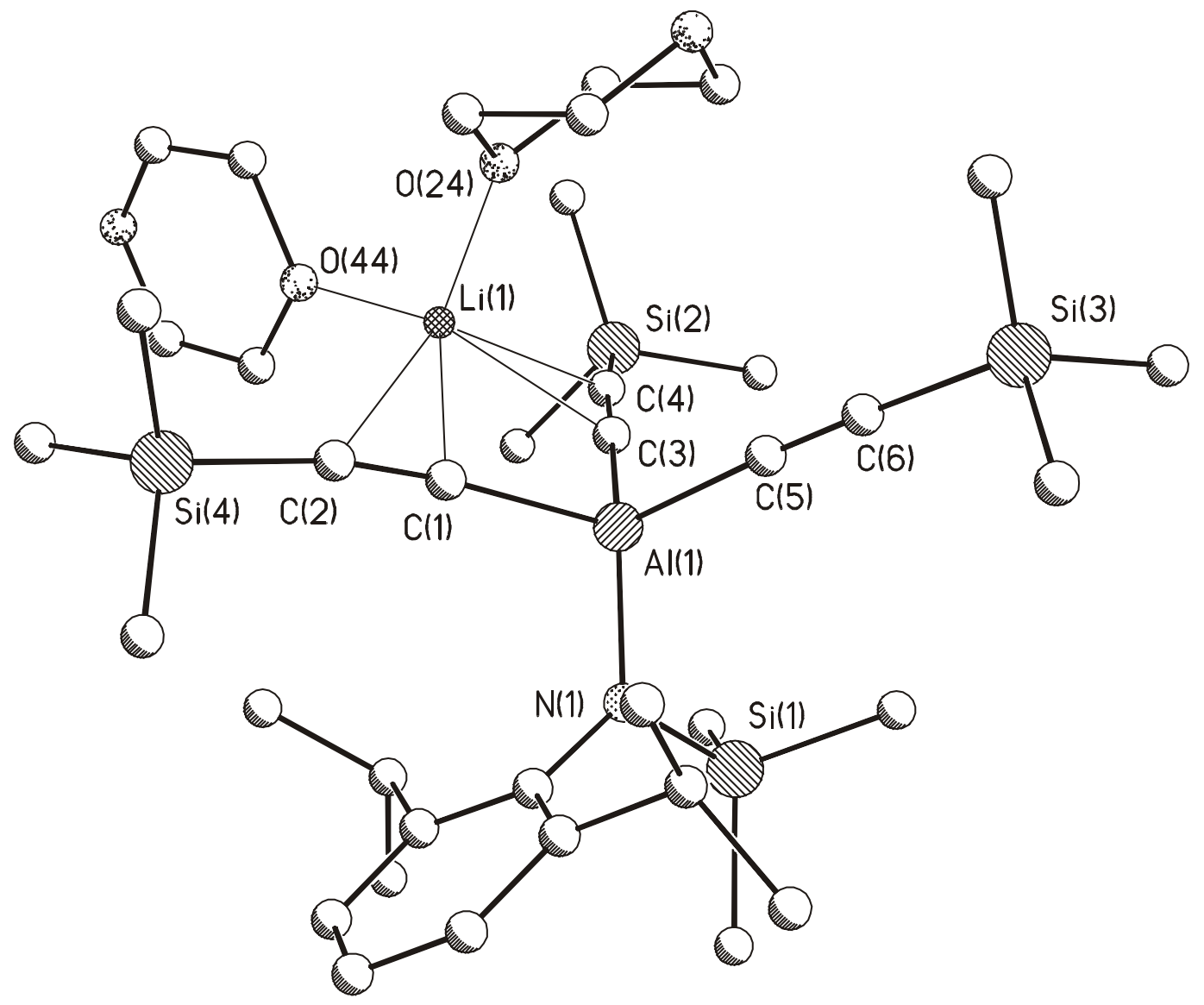

Abb. 6: Struktur von 7 im Kristall. Die Dioxanmoleküle im Gitter sind der Übersichtlichkeit halber nicht dargestellt.

Ausgewählte Bindungslängen $(\AA ̊)$ und Winkel ( $\left.{ }^{\circ}\right)$ für 7.

$\begin{array}{llll}\mathrm{Al}(1)-\mathrm{C}(5) & 1.947(4) & \mathrm{Al}(1)-\mathrm{N}(1) & 1.837(3) \\ \mathrm{Al}(1)-\mathrm{C}(1) & 1.972(5) & \mathrm{C}(5)-\mathrm{Al}(1)-\mathrm{C}(1) & 109.29(18) \\ \mathrm{Al}(1)-\mathrm{C}(3) & 1.979(4) & \mathrm{C}(5)-\mathrm{Al}(1)-\mathrm{C}(3) & 108.90(18) \\ \mathrm{Li}(1)-\mathrm{C}(3) & 2.333(9) & \mathrm{C}(1)-\mathrm{Al}(1)-\mathrm{C}(3) & 103.04(18) \\ \mathrm{Li}(1)-\mathrm{C}(1) & 2.343(9) & \mathrm{C}(1)-\mathrm{Li}(1)-\mathrm{C}(2) & 25.44(15) \\ \mathrm{Li}(1)-\mathrm{C}(4) & 2.734(9) & \mathrm{C}(3)-\mathrm{Li}(1)-\mathrm{C}(4) & 26.26(16) \\ \mathrm{Li}(1)-\mathrm{C}(2) & 2.786(9) & \mathrm{C}(4)-\mathrm{C}(3)-\mathrm{Al}(1) & 176.6(4) \\ \mathrm{C}(3)-\mathrm{C}(4) & 1.215(6) & \mathrm{C}(2)-\mathrm{C}(1)-\mathrm{Al}(1) & 175.0(4) \\ \mathrm{C}(2)-\mathrm{C}(1) & 1.209(6) & \mathrm{C}(6)-\mathrm{C}(5)-\mathrm{Al}(1) & 177.3(4) \\ \mathrm{C}(5)-\mathrm{C}(6) & 1.205(6) & & \end{array}$


Vergleicht man die Verbindungen [Li · 2 Dioxan $\left.\left(2,6-i \mathrm{Pr}_{2} \mathrm{C}_{6} \mathrm{H}_{3} \mathrm{~N}\left(\mathrm{SiMe}_{3}\right) \mathrm{Al}\left(\mathrm{C} \equiv \mathrm{CSiMe}_{3}\right)_{3}\right)\right]$. 0.75 Dioxan (7) mit [ Li · Dioxan (2,6-i $\left.\left.\mathrm{Pr}_{2} \mathrm{C}_{6} \mathrm{H}_{3} \mathrm{~N}\left(\mathrm{SiMe}_{3}\right) \mathrm{Al}(\mathrm{C} \equiv \mathrm{CPh})_{3}\right)\right]_{2} \cdot 2$ Dioxan (6) so zeigt sich ein fast gleicher Aufbau. Jedoch fungiert bei 6 ein Dioxan-Molekül als verbrückende Einheit, während dies bei 7 nicht der Fall ist. Bei beiden Verbindungen ist das Lithium-Kation von zwei Ethinyl-Liganden sowie von zwei Dioxan-Molekülen koordiniert. Der Abstand der Al-C-Bindung (1.947-1.979 $\AA$ ) in 7 ist mit denen der Verbindungen 4, 5 und 6 vergleichbar. Auch der Abstand des Lithium-Kations zu den in $\alpha$-Position zum Aluminium gebundenen Ethinyl-Kohlenstoffatomen (2.333 und $2.343 \AA$ ) kann mit dem Abstand bei Verbindung 6 (2.287 und $2.350 \AA$ ) verglichen werden.

Darüber hinaus existieren jedoch auch Unterschiede. Besonders auffällig ist der deutlich verlängerte Bindungsabstand des Lithium-Kations zu den in $\beta$-Position zum Aluminiumatom gebundenen Alkinyl-Kohlenstoffatomen (2.734 und $2.768 \AA$ (7) verglichen mit 2.464 und $2.594 \AA$ (6)). Dies kann mit der größeren sterischen Abstoßung der $\mathrm{SiMe}_{3}$-Gruppen erklärt werden.

Die Al-C $\equiv \mathrm{C}$-Bindungswinkel $\left(176.6^{\circ}, 175.0^{\circ}, 177.3^{\circ}\right)$ weichen hier nicht so stark von der Linearität ab wie z.B. der Al-C(3)-C(30)-Winkel in 6 mit $166.6^{\circ}$.

Die etwas kleineren C-Li-C-Winkel $\left(25.44^{\circ}\right.$ und $\left.26.26^{\circ}\right)$ in 7 als in Verbindung $6\left(27.76^{\circ}\right.$ und $29.21^{\circ}$ ) lassen sich durch den größeren Abstand des $\beta$-C-Atoms (s.o.) erklären.

\subsubsection{Versuche zur Darstellung von $\mathrm{RAI}\left(\mathrm{C} \equiv \mathrm{CSiMe}_{3}\right)_{2}$}

$\left(\right.$ mit $\left.\mathrm{R}=2,6-i \mathrm{Pr}_{2} \mathrm{C}_{6} \mathrm{H}_{3} \mathrm{~N}\left(\mathrm{SiMe}_{3}\right)\right)$

Die Ergebnisse zeigen, dass die Aluminium-Verbindung 7 mit drei terminalen EthinylLiganden des Typs $\mathrm{M} \cdot$ Solvens $\left[\mathrm{RAl}\left(\mathrm{C} \equiv \mathrm{CSiMe}_{3}\right)_{3}\right]$ die thermodynamisch stabilere Verbindung verglichen mit der theoretisch ebenfalls möglichen neutralen Aluminium-DiethinylVerbindung mit nur zwei terminalen Ethinyl-Liganden des Typs $\left[\mathrm{RAl}\left(\mathrm{C} \equiv \mathrm{CSiMe}_{3}\right)_{2} \cdot \mathrm{THF}\right]$ ist. Denn auch beim Einsatz eines großen, sterisch anspruchsvollen Restes an der Ethinyl-Gruppe entsteht eine Verbindung mit vierfach koordiniertem Aluminiumatom, die drei EthinylLiganden gebunden hat. 
Dass es sich um die bevorzugt gebildete Verbindung handelt, zeigt u.a. folgendes Ergebnis:

Ein Versuch, $\left[\mathrm{RAlCl}_{2}\right]_{2}$ (3) mit nur vier Äquivalenten $\mathrm{LiC} \equiv \mathrm{CSiMe}_{3}$ umzusetzen, ergab eine Mischung aus mehreren Verbindungen, aus der nur das bekannte dreifach substituierte Produkt isoliert werden konnte.

$1 / 2$

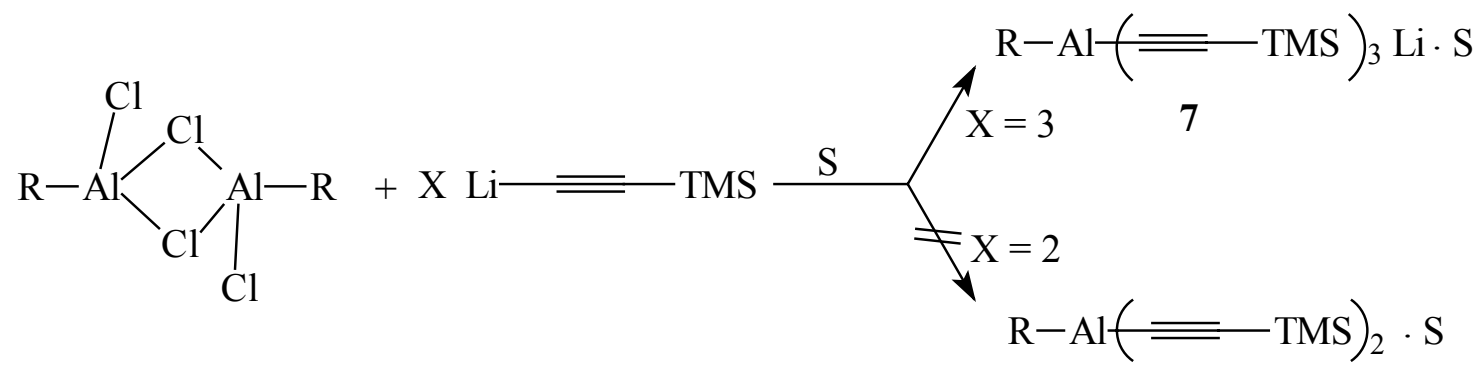

3

Schema 20: $\quad \mathrm{R}=2,6-i \mathrm{Pr}_{2} \mathrm{C}_{6} \mathrm{H}_{3} \mathrm{~N}\left(\mathrm{SiMe}_{3}\right) \mathrm{S}=\mathrm{THF}$, Dioxan.

Ein weiterer Beleg dafür, dass es sich bei 7 um eine sehr stabile Verbindung handelt, zeigen die Versuche, Verbindung 7 mit Methyliodid oder TMSCl umzusetzen.

$\mathrm{Li} \mathrm{R}-\mathrm{Al}(\overline{=} \mathrm{TMS})_{3}+\mathrm{MeI} \underset{-\mathrm{LiI}}{\stackrel{(f \longrightarrow}{\longrightarrow}} \mathrm{R}-\mathrm{Al}(\overline{=} \mathrm{TMS})_{2}+\mathrm{Me}=\mathrm{TMS}$

Schema 21: Versuch zur Darstellung einer neutralen Aluminium-Diethinyl-Verbindung des Typs $\mathrm{RAl}(\mathrm{C} \equiv \mathrm{CR})_{2}$ mit zwei terminalen Ethinyl-Liganden aus 7.

Bei diesen Umsetzungen wurden Rückstände erhalten, die weder durch Umkristallisation noch durch Sublimation weiter aufgetrennt werden konnten. 


\subsubsection{Darstellung von $\left[\mathrm{Li} \cdot \mathrm{Et}_{2} \mathrm{O}\left(2,6-i \mathrm{Pr}_{2} \mathrm{C}_{6} \mathrm{H}_{3} \mathrm{~N}\left(\mathrm{SiMe}_{3}\right) \mathrm{GaCl}_{3}\right)\right](8)$}

$\mathrm{Zu}$ 2,6-i $\mathrm{Pr}_{2} \mathrm{C}_{6} \mathrm{H}_{3} \mathrm{~N}\left(\mathrm{SiMe}_{3}\right) \mathrm{Li}$ in Diethylether wurde bei $-78{ }^{\circ} \mathrm{C}$ eine Lösung von Galliumtrichlorid in Diethylether gegeben und die Mischung vier Stunden lang bei Raumtemperatur gerührt. Nach Extraktion mit warmem Trichlormethan konnte 8 als hellgelber Feststoff erhalten werden.
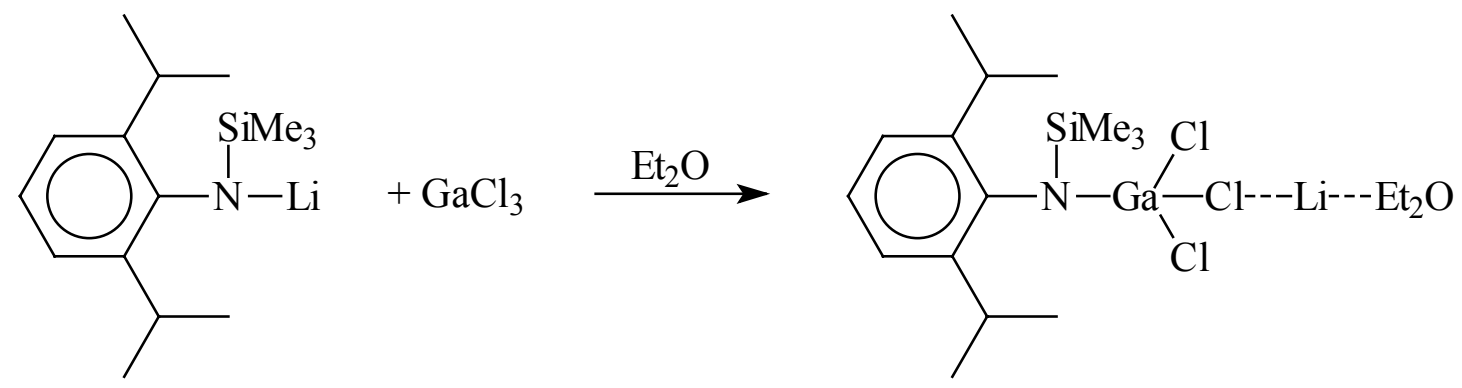

8

Schema 22: Darstellung von $\left[\mathrm{Li} \cdot \mathrm{Et}_{2} \mathrm{O}\left(2,6-i \mathrm{Pr}_{2} \mathrm{C}_{6} \mathrm{H}_{3} \mathrm{~N}\left(\mathrm{SiMe}_{3}\right) \mathrm{GaCl}_{3}\right)\right](\mathbf{8})$.

Da die homologe Indiumverbindung schon strukturell charakterisiert wurde, ${ }^{[56]}$ konnte auf eine Einkristallstrukturanalyse verzichtet werden. Dass es sich bei dieser Verbindung ebenfalls um ein Lithiumchlorid-Addukt handelt, ist aus dem ${ }^{7}$ Li-NMR-Spektrum zu schließen. Es lässt sich eine Resonanz bei $\delta-0.4$ beobachten, welche sich dem von Diethylether koordinierten Lithium-Ion zuordnen lässt. Das ${ }^{1} \mathrm{H}-\mathrm{NMR}-$ Spektrum zeigt ein Multiplett bei $\delta 7.20$ - 6.80 für die drei aromatischen Protonen und ein Septett für die beiden Protonen der $\mathrm{CH}$-Gruppe, welche mit den Protonen der benachbarten $\mathrm{CH}_{3}$-Gruppen koppeln, die wiederum jeweils als Dublett bei $\delta 1.14$ und $1.13 \mathrm{zu}$ sehen sind. Dass es sich um ein einfach koordiniertes Diethylether-Addukt handelt, welches an das Lithium-Ion gebunden ist, ist aus der Intensität und Lage der Resonanzen des Diethylethers $\left(\delta=3.38\right.$ für die $\mathrm{O}-\mathrm{CH}_{2}$ Gruppen und $\delta 1.11$ für die $\mathrm{CH}_{3}$-Gruppen) abzuleiten. Im Massenspektrum ist der molare Massen-Peak (389) der Verbindung ohne das koordinierende Lithiumchlorid und den Diethylether zu sehen. Die Elementaranalyse bestätigt die Zusammensetzung $\mathrm{C}_{19} \mathrm{H}_{36} \mathrm{Cl}_{3} \mathrm{GaLiNOSi}$. 


\subsubsection{Darstellung von}

$\left[(\mathrm{Li})_{2} \cdot(\operatorname{Dioxan})_{7}\right]_{0.5}\left[2,6-i \mathrm{Pr}_{2} \mathrm{C}_{6} \mathrm{H}_{3} \mathrm{~N}\left(\mathrm{SiMe}_{3}\right) \mathrm{Ga}\left(\mathrm{C} \equiv \mathrm{CSiMe}_{3}\right)_{3}\right] \cdot 1.5 \operatorname{Dioxan}(9)$

8 wurde mit drei Äquivalenten $\mathrm{LiC} \equiv \mathrm{CSiMe}_{3}$ in $\mathrm{THF}$ bei $-78^{\circ} \mathrm{C}$ versetzt und 24 Stunden lang bei Raumtemperatur gerührt. Die Lösungsmittel wurden im Vakuum entfernt, der Rückstand in Toluol aufgenommen, über Celite filtriert und das Filtrat mit Dioxan versetzt. Nach 24 Stunden wurden die Lösungsmittel im Vakuum entfernt und der Rückstand in Toluol gelöst. Die Lösung wurde zwei Tage lang bei $-32{ }^{\circ} \mathrm{C}$ gelagert, bis das Produkt vollständig auskristallisiert war. Nach Filtration und Trocknen des Produkts im Vakuum erhielt man 9, die erste anionische Gallium-Verbindung bei der drei terminale Ethinyl-Liganden an das Galliumzentrum gebunden sind. Ein von Dioxan koordiniertes Lithiumion fungiert als Gegenion.

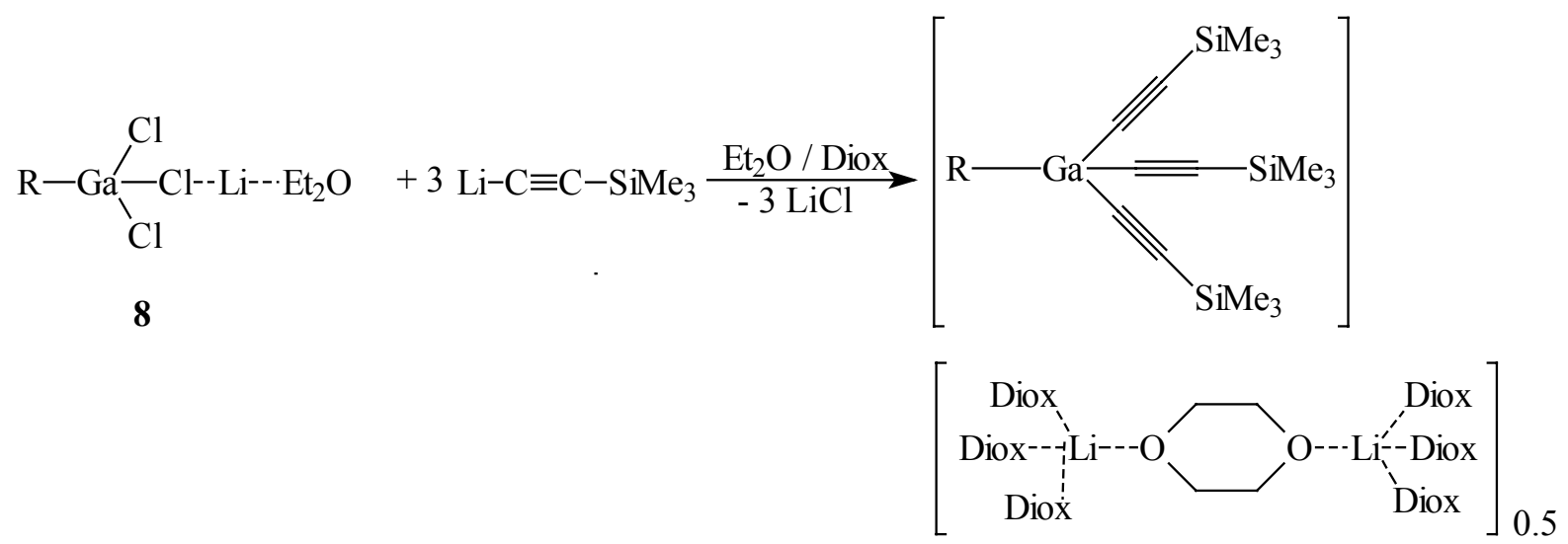

9

Schema 23: Darstellung von $9\left(\mathrm{R}=2,6-i \mathrm{Pr}_{2} \mathrm{C}_{6} \mathrm{H}_{3} \mathrm{~N}\left(\mathrm{SiMe}_{3}\right)\right.$; Diox $=$ Dioxan $)$.

Das ${ }^{1} \mathrm{H}-\mathrm{NMR}$ zeigt bei $\delta-0.03$ und 0.07 zwei scharfe Singuletts im Verhältnis 9:27 und lässt

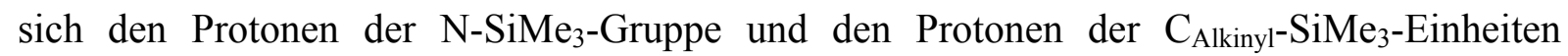
zuordnen. Die übrigen Resonanzen des ${ }^{1}$ H-NMR-Spektrums lassen sich den Protonen des 2,6$i \operatorname{Pr}_{2} \mathrm{C}_{6} \mathrm{H}_{3} \mathrm{~N}\left(\mathrm{SiMe}_{3}\right)$-Substituenten sowie dem Dioxan zuordnen. Anhand der Resonanzintensität der Dioxan-Protonen sieht man, dass das im Vakuum getrocknete Produkt nur 2 DioxanMoleküle koordiniert hat, während im Kristall fünf Dioxan-Moleküle pro Elementarzelle koordinieren. Im ${ }^{13} \mathrm{C}$-NMR-Spektrum ist die Resonanz der in $\beta$-Position zum Gallium gebundenen Ethinyl-Kohlenstoffatomen bei $\delta 95.2 \mathrm{zu}$ sehen sowie ein breites Signal der an das Gallium gebundenen Kohlenstoffatome bei $\delta$ 107.4. Die zu den beiden $\mathrm{SiMe}_{3}$-Gruppen 
gehörenden ${ }^{29} \mathrm{Si}-\mathrm{NMR}-$ Resonanzen liegen bei $\delta-4.2$ und -25.2. Das ${ }^{7}$ Li-NMR zeigt ein Signal bei $\delta-0.5$ und lässt sich dem von Dioxan koordinierten Lithium-Kation zuordnen.

Die bei der Elementaranalyse gefundenen Werte bestätigen die Zusammensetzung der von zwei Dioxan-Molekülen koordinierten Gallium-Verbindung $\mathrm{C}_{38} \mathrm{H}_{6} \mathrm{NSi}_{4} \mathrm{GaLiO}_{4}$.

Die gut lösliche Verbindung zersetzte sich jedoch nach mehreren Tagen zu Produkten, die nicht identifiziert werden konnten.

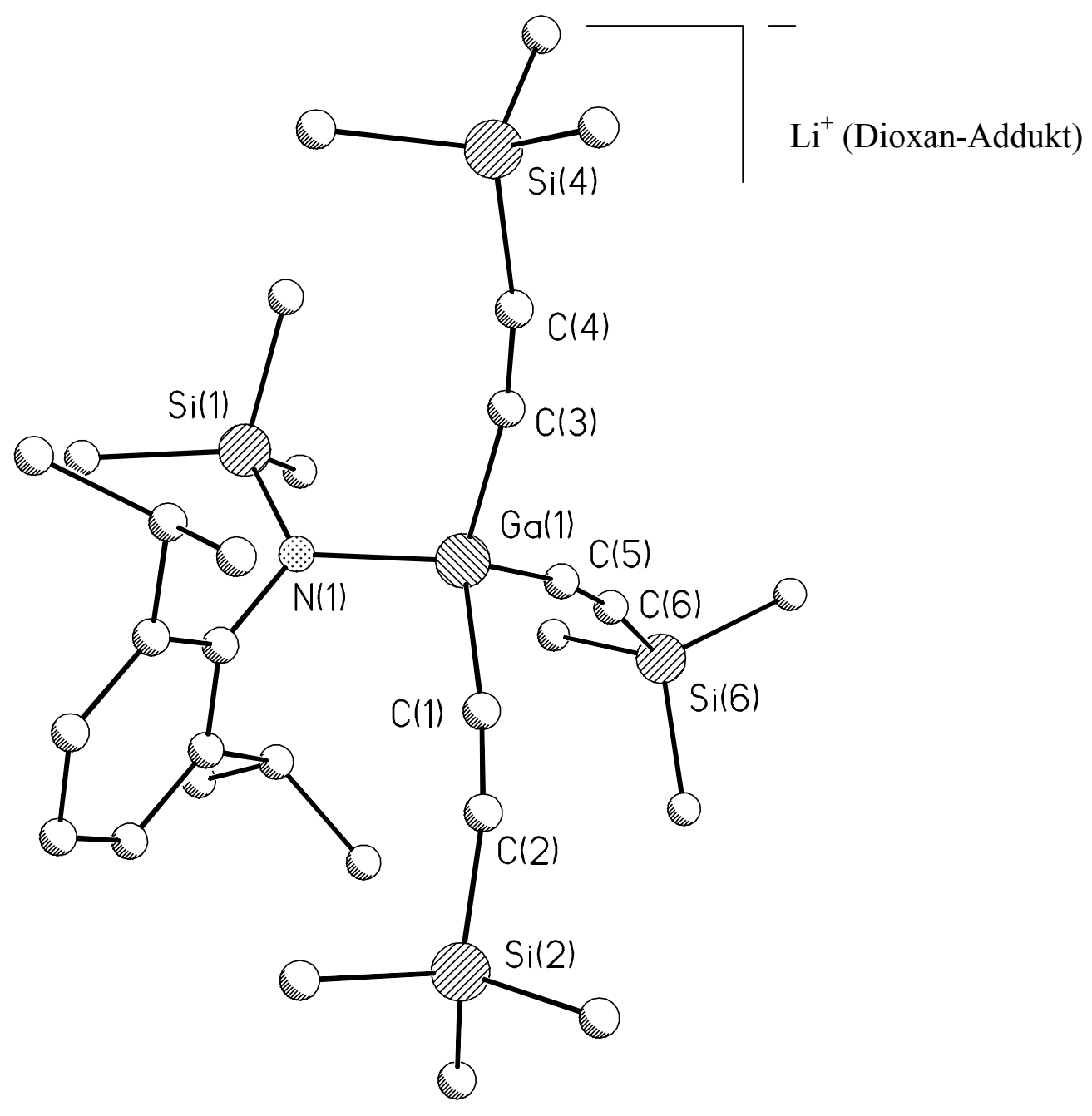

Abb. 7: Struktur des Anions von 9 im Kristall.

Ausgewählte Bindungslängen $(\AA)$ und Winkel $\left(^{\circ}\right)$ für 9.

$\begin{array}{llll}\mathrm{Ga}(1)-\mathrm{C}(1) & 1.966(8) & \mathrm{C}(1)-\mathrm{Ga}(1)-\mathrm{C}(5) & 107.4(3) \\ \mathrm{Ga}(1)-\mathrm{C}(5) & 1.970(8) & \mathrm{C}(1)-\mathrm{Ga}(1)-\mathrm{C}(3) & 111.8(3) \\ \mathrm{Ga}(1)-\mathrm{C}(3) & 1.971(8) & \mathrm{C}(5)-\mathrm{Ga}(1)-\mathrm{C}(3) & 111.8(3) \\ \mathrm{C}(1)-\mathrm{C}(2) & 1.227(10) & \mathrm{C}(2)-\mathrm{C}(1)-\mathrm{Ga}(1) & 173.7(6) \\ \mathrm{C}(3)-\mathrm{C}(4) & 1.205(10) & \mathrm{C}(4)-\mathrm{C}(3)-\mathrm{Ga}(1) & 169.9(6) \\ \mathrm{C}(5)-\mathrm{C}(6) & 1.226(10) & \mathrm{C}(6)-\mathrm{C}(5)-\mathrm{Ga}(1) & 170.9(6)\end{array}$


Die Röntgenstrukturanalyse zeigt, dass der Abstand der Ga-C $\mathrm{C}_{\text {Alkinyl-Bindungen zwischen }}$ $1.966 \AA$ und 1.971 Å liegt. Dies entspricht den Erwartungen (vgl. Tab. 3). ${ }^{[57,58,59,60]}$

Die Ga-C $\equiv \mathrm{C}-$ Winkel weichen von $180^{\circ} \mathrm{ab}\left(169.9^{\circ}, 170.9^{\circ}, 173.7^{\circ}\right)$. Diese Abweichung ist bei allen drei Ethinyl-Liganden größer als in der entsprechenden Aluminium-Verbindung (7) $\left(175.0^{\circ}-177.3^{\circ}\right)$. Ein weiterer Unterschied zur Aluminiumverbindung 7 ist, dass hier das Lithium-Ion nicht von den Ethinylgruppen, sondern von den Lösungsmittel-Molekülen (Dioxan) koordiniert wird.

Im Vergleich zu den bisher beschriebenen Gallium-Ethinyl-Verbindungen ${ }^{[57,58,59,60]}$ findet man in Verbindung 9 kleine Ga-C $\equiv \mathrm{C}$-Winkel $\left(169.9^{\circ}, 170.9^{\circ}, 173.7^{\circ}\right)$.

Zum Vergleich:

Tabelle 3: Alle strukturell charakterisierten Gallium-Ethinyl-Produkte.

\begin{tabular}{|c|c|c|c|}
\hline & $\begin{array}{l}\text { Ga-C }{ }_{\text {Alkinyl- }} \\
\text { Bindungs- } \\
\text { länge }(\AA ̊)\end{array}$ & $\begin{array}{l}\mathrm{C} \equiv \mathrm{C}- \\
\text { Bindungs- } \\
\text { länge }(\AA)\end{array}$ & $\begin{array}{l}\text { Ga-C } \equiv C- \\
\text { Bindungs- } \\
\text { winkel }\end{array}$ \\
\hline$[(\text { Tetraphenylporphyrin }) \mathrm{Ga}(\mathrm{C} \equiv \mathrm{C} n \mathrm{Pr})]^{[57]}$ & 1.949 & 1.196 & $178.8^{\circ}$ \\
\hline trans $\left.-\left[(t \mathrm{Bu}) \mathrm{Me}_{3} \mathrm{SiC} \equiv \mathrm{C}\right) \mathrm{GaPEt}_{2}\right]_{2}^{[58]}$ & 1.936 & 1.190 & $168.8^{\circ}$ \\
\hline$\left[\mathrm{Me}_{2} \mathrm{GaC} \equiv \mathrm{CPh}\right]_{2}^{[59]}$ & 2.004 & 1.183 & $172.8^{\circ}$ \\
\hline $\begin{array}{l}{\left[\mathrm{Li}(\mathrm{THF})_{4}\right]} \\
{\left[\left(\left(\mathrm{SiMe}_{3}\right)_{2} \mathrm{CH}\right)_{2} \mathrm{Ga}-\mathrm{Ga}\left(\left(\mathrm{SiMe}_{3}\right)_{2} \mathrm{CH}\right)_{2}(\mathrm{C} \equiv \mathrm{CPh})\right]^{[60]}}\end{array}$ & 2.007 & 1.200 & $177.3^{\circ}$ \\
\hline
\end{tabular}

Verbindungen 7 und 9 unterscheiden sich im Aufbau des Anions lediglich im Zentralatom. Das Kation wird jedoch unterschiedlich in den beiden Verbindungen koordiniert. Während es in 7 von den Ethinyl-Liganden koordiniert wird, koordinieren in 9 nur Dioxan-Moleküle an das Lithium-Kation. Entscheidend für die Koordination des Lithiums ist das Zentralatom. Die Beobachtung, dass bei Verbindung 9 nun das "Pearson-harte" Lithium-Ion an die "Pearsonharten" Sauerstoffatome des Dioxans koordiniert, entspricht den Erwartungen. 


\subsubsection{Darstellung von}

$\left[(\mathrm{Li})_{2} \cdot(\text { Dioxan })_{7}\right]_{0.5}\left[2,6-i \operatorname{Pr}_{2} \mathrm{C}_{6} \mathrm{H}_{3} \mathrm{~N}\left(\mathrm{SiMe}_{3}\right) \operatorname{In}\left(\mathrm{C} \equiv \mathrm{CSSiMe}_{3}\right)_{3}\right] \cdot 1.5$ Dioxan $(10)$

Bei der Umsetzung von $\left[\mathrm{Li} \cdot 3 \mathrm{THF}\left(2,6-i \mathrm{Pr}_{2} \mathrm{C}_{6} \mathrm{H}_{3} \mathrm{~N}\left(\mathrm{SiMe}_{3}\right) \mathrm{InCl}_{3}\right)\right]^{[56]}$ mit drei Äquivalenten $\mathrm{LiC} \equiv \mathrm{CSiMe}_{3}$ in Toluol und anschließender Zugabe von Dioxan erhielt man nach Entfernen des Lösungsmittels im Vakuum die erste anionische Indiumverbindung, bei der drei terminale Ethinyl-Liganden an Indium gebunden sind. Ein von Dioxan koordiniertes Lithium-Kation fungiert als Gegenion. Anhand der spektroskopischen Untersuchung sowie der Farbveränderung des Produktes konnte man erkennen, dass sich die gut lösliche Verbindung selbst bei Lagerung bei $-32{ }^{\circ} \mathrm{C}$ nach einigen Tagen zu Produkten zersetzte, die nicht identifiziert werden konnten.

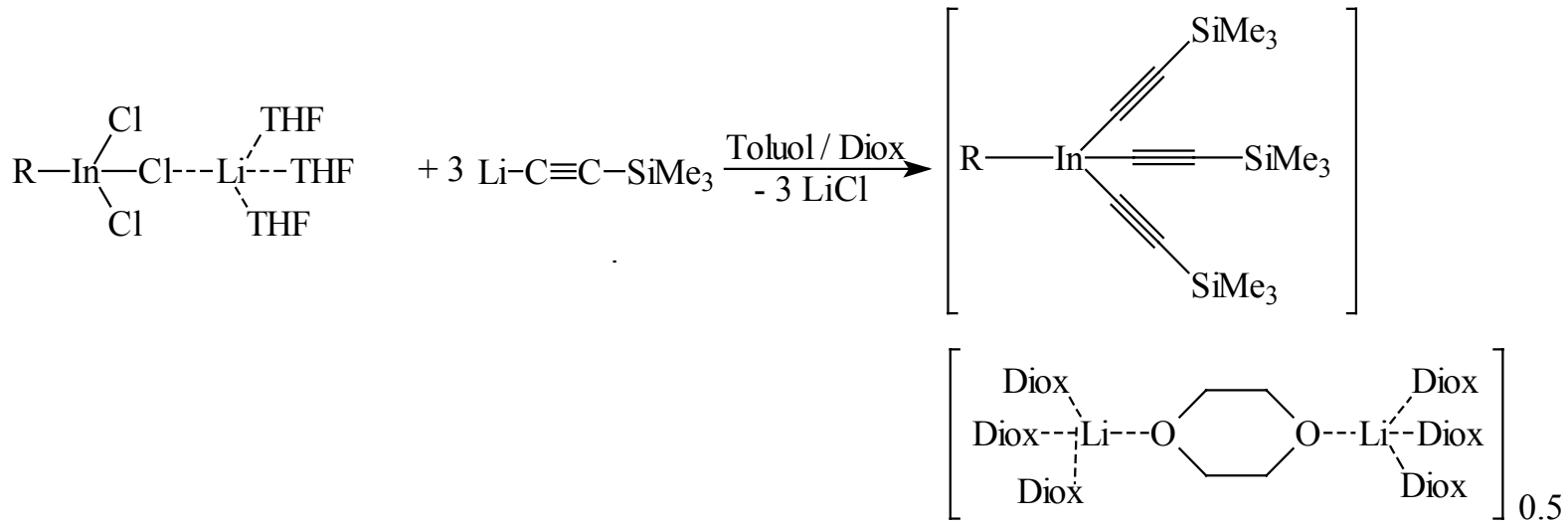

10

Schema 24: Darstellung von $10\left(\mathrm{R}=2,6-i \mathrm{Pr}_{2} \mathrm{C}_{6} \mathrm{H}_{3} \mathrm{~N}\left(\mathrm{SiMe}_{3}\right)\right.$; Diox = Dioxan $)$

Dem ${ }^{1}$ H-NMR-Spektrum ist anhand der Signalintensität der Dioxan-Moleküle zu entnehmen, dass im Unterschied zu dem Aufbau im Kristall nach Anlegen des Vakuums nur noch zwei Dioxan-Moleküle koordiniert sind. Außer den bekannten Resonanzen des 2,6-i $\operatorname{Pr}_{2} \mathrm{C}_{6} \mathrm{H}_{3} \mathrm{~N}\left(\mathrm{SiMe}_{3}\right)$-Liganden sind noch die Resonanzen der an die AlkinylKohlenstoffatome gebundenen $\mathrm{SiMe}_{3}$-Protonen zu sehen. Im ${ }^{29} \mathrm{Si}$-NMR-Spektrum sieht man die Resonanz dieser Atome bei $\delta-25.2$ und ein Signal bei $\delta-4.5$, welches den ${ }^{29} \mathrm{Si}-\mathrm{Kernen}$ der $\mathrm{SiMe}_{3}$-Gruppe des Liganden zugeordnet werden kann. Die ${ }^{7} \mathrm{Li}$-Kerne erzeugen im NMR eine Resonanz bei $\delta$-0.2. Im ${ }^{13} \mathrm{C}-\mathrm{NMR}$ ist das Signal, welches die an das Indium gebundenen Alkinyl-Kohlenstoffatome bei $\delta 114.1$ erzeugen aufgrund ihres breiten Erscheinungsbildes leicht zuzuordnen (breites Erscheinungsbild aufgrund des Quadrupol-Effekts, vgl. Kapitel 
4.2.1.) während die in $\beta$-Position zum Indium gebundene Ethinyl-Kohlenstoffatome ein Signal bei $\delta 95.2$ erzeugen.

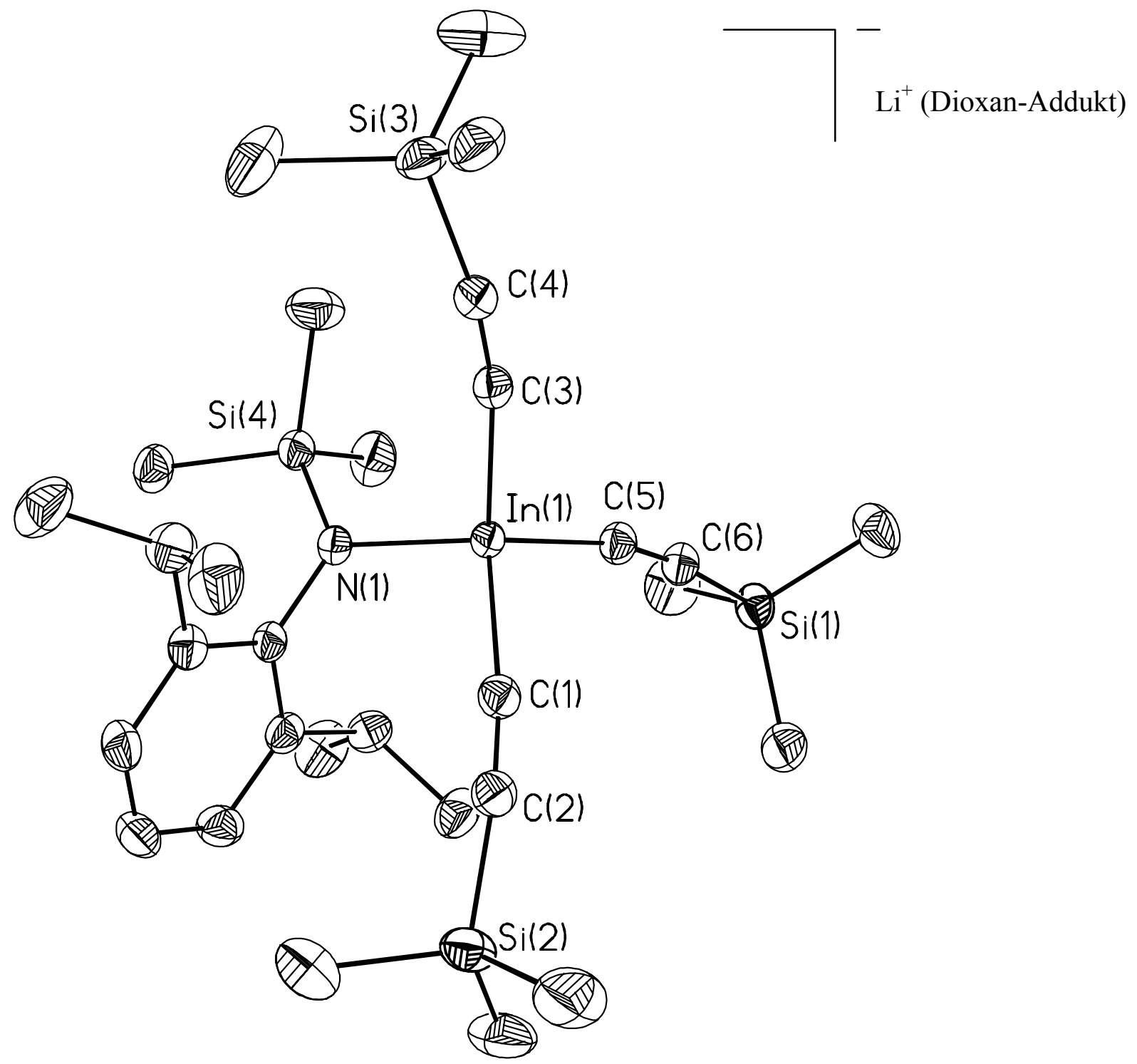

Abb. 8: Struktur des Anions von 10 im Kristall.

Ausgewählte Bindungslängen $(\AA)$ und Winkel $\left(^{\circ}\right)$ für 10.

$\begin{array}{llll}\operatorname{In}(1)-\mathrm{N}(1) & 2.113(2) & \mathrm{N}(1)-\operatorname{In}(1)-\mathrm{C}(3) & 108.70(7) \\ \operatorname{In}(1)-\mathrm{C}(1) & 2.148(2) & \mathrm{C}(1)-\operatorname{In}(1)-\mathrm{C}(3) & 112.52(7) \\ \operatorname{In}(1)-\mathrm{C}(3) & 2.154(2) & \mathrm{N}(1)-\operatorname{In}(1)-\mathrm{C}(5) & 110.06(6) \\ \operatorname{In}(1)-\mathrm{C}(5) & 2.169(2) & \mathrm{C}(1)-\operatorname{In}(1)-\mathrm{C}(5) & 106.90(7) \\ \mathrm{C}(5)-\mathrm{C}(6) & 1.210(3) & \mathrm{C}(3)-\operatorname{In}(1)-\mathrm{C}(5) & 112.49(7) \\ \mathrm{C}(1)-\mathrm{C}(2) & 1.206(3) & \mathrm{C}(6)-\mathrm{C}(5)-\operatorname{In}(1) & 168.10(15) \\ \mathrm{C}(3)-\mathrm{C}(4) & 1.207(3) & \mathrm{C}(2)-\mathrm{C}(1)-\operatorname{In}(1) & 170.92(16) \\ \mathrm{N}(1)-\operatorname{In}(1)-\mathrm{C}(1) & 105.96(7) & \mathrm{C}(4)-\mathrm{C}(3)-\operatorname{In}(1) & 168.97(15)\end{array}$


Die Röntgenstrukturanalyse von 10 zeigt, dass der Abstand der In- $\mathrm{C}_{\text {Alkinyl}}$-Bindungen, der zwischen $2.148 \AA$ und $2.169 \AA$ liegt, sowie der $\mathrm{C} \equiv \mathrm{C}$-Abstand $(1.21 \AA)$ den Erwartungen entspricht (vgl. Tab. 4), während die In-C $\equiv \mathrm{C}-$ Winkel $\left(168.10^{\circ} ; 170.92^{\circ} ; 168.97^{\circ}\right)$ die kleinsten gemessenen sind. ${ }^{[60,61,62,63,64]}$

Tabelle 4: Alle strukturell charakterisierten Indium-Ethinyl-Verbindungen

\begin{tabular}{|c|c|c|c|}
\hline & $\begin{array}{l}\text { In-C } \text { Alkinyl- } \\
\text { Bindungs- } \\
\text { länge(n) }(\AA)\end{array}$ & $\begin{array}{l}\mathrm{C} \equiv \mathrm{C}- \\
\text { Bindungs- } \\
\text { länge(n) }(\AA)\end{array}$ & 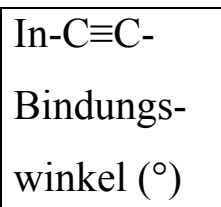 \\
\hline $\begin{array}{l}{\left[\mathrm{Li}(\mathrm{THF})_{4}\right]} \\
{\left[\left(\left(\mathrm{SiMe}_{3}\right)_{2} \mathrm{CH}\right)_{2} \mathrm{In}-\mathrm{In}\left(\left(\mathrm{SiMe}_{3}\right)_{2} \mathrm{CH}\right)_{2}(\mathrm{C} \equiv \mathrm{CPh})\right]^{[60]}}\end{array}$ & 2.192 & 1.193 & 176.2 \\
\hline$\left[\mathrm{Li}(\mathrm{THF})_{4}\right]\left[\operatorname{In}(\mathrm{C} \equiv \mathrm{C}-\mathrm{Ph})_{4}\right]^{[6 \mathrm{~T}}$ & $2.155-2.165$ & $1.194-1.204$ & $171.2-179.0$ \\
\hline $\mathrm{In}\left(\mathrm{C} \equiv \mathrm{C}-\mathrm{CF}_{3}\right)_{3} \cdot 2 \mathrm{THF}^{[62]}$ & $2.11-2.13$ & $1.20-1.24$ & $172-180.0$ \\
\hline$(\mathrm{Dipp})_{2} \mathrm{NacNacIn}(\mathrm{C} \equiv \mathrm{C}-\mathrm{Ph})_{2}{ }^{[63]}$ & $2.117,2.118$ & $1.200,1.201$ & $171.2,171.5$ \\
\hline $\mathrm{Me}_{2} \operatorname{In}(\mathrm{C} \equiv \mathrm{C}-\mathrm{Me})^{\lfloor 64\rfloor}$ & 2.193 & 1.211 & 177.1 \\
\hline
\end{tabular}

Die In-C $\equiv \mathrm{C}$-Winkel weichen damit stärker als die Ga-C $\equiv \mathrm{C}$-Winkel von $180^{\circ}$ ab $\left(\mathrm{Ga}: 169.9^{\circ}\right.$, $\left.170.9^{\circ}, 173.7^{\circ}\right)$. Die Abweichung ist bei allen drei Ethinyl-Liganden, wie schon bei der Gallium-Verbindung 9, ähnlich groß und ebenfalls größer als in der entsprechenden Aluminium-Verbindung (7) $\left(175.0^{\circ}-177.3^{\circ}\right)$.

Die Abweichung von dem idealen $\mathrm{M}-\mathrm{C} \equiv \mathrm{C}-$ Winkel von $180^{\circ}$ nimmt damit vom Aluminium über Gallium zum Indium zu.

Wie schon bei 9 und im Unterschied zu 7 ist hier das Lithium-Ion nicht an die Ethinyl-Gruppe gebunden, sondern es wird von Lösungsmittel-Molekülen (Dioxan) koordiniert. Dies entspricht den Erwartungen. ${ }^{[36]}$

\subsubsection{Darstellung der Verbindung}

$\left[\mathrm{Li} \cdot 2\right.$ THF (2,6-iPr $\left.\left.{ }_{2} \mathrm{C}_{6} \mathrm{H}_{3} \mathrm{~N}\left(\mathrm{SiMe}_{3}\right) \operatorname{In}\left(\mathrm{C} \equiv \mathrm{CSiMe}_{3}\right)_{3}\right)\right](11)$

Eine Verbindung, bei der die Struktur des Anions identisch mit der von 10 ist, wird bei der Umsetzung von Verbindung [Li 3 THF (2,6-i $\left.\left.\operatorname{Pr}_{2} \mathrm{C}_{6} \mathrm{H}_{3} \mathrm{~N}\left(\mathrm{SiMe}_{3}\right) \mathrm{InCl}_{3}\right)\right]^{[56]}$ mit drei Äquivalenten $\mathrm{LiC} \equiv \mathrm{CSiMe}_{3}$ in Toluol/ THF erhalten. Nach Filtration und anschließender Lagerung des Filtrats bei $-30{ }^{\circ} \mathrm{C}$ bildeten sich nach drei Tagen farblose Kristalle. Nach Entnahme eines 
Einkristalls, Filtration und Trocknung des Rückstands wurde $\mathbf{1 1}$ als farblose Verbindung erhalten. Wie $\mathbf{1 0}$ zersetzt sich $\mathbf{1 1}$ bereits nach einigen Tagen zu nicht identifizierbaren Produkten.

Im ${ }^{1}$ H-NMR-Spektrum sind neben den Protonen-Signalen der zwei THF-Moleküle und des Liganden auch die Protonen-Signale der $\mathrm{SiMe}_{3}$-Gruppen an den Alkinyl-Kohlenstoffatomen zu beobachten. Das ${ }^{7}$ Li-NMR zeigt ein Signal bei $\delta-0.3$, welches dem von den EthinylGruppen koordinierten Lithium-Kation zuzuordnen ist. Das ${ }^{13} \mathrm{C}-\mathrm{NMR}$ zeigt die AlkinylKohlenstoffatome, welche an das Indium gebunden sind bei $\delta 145.6$ aufgrund des QuadrupolEffektes als sehr breites Signal, während die in $\beta$-Position zum Indium gebundenen Kohlenstoffatome ein scharfes Signal bei $\delta 95.2$ erzeugen. Das ${ }^{29}$ Si-NMR zeigt ein Signal bei $\delta$-6.5, welches den ${ }^{29} \mathrm{Si}-$ Kernen des Liganden zuzuordnen sind, sowie eine Resonanz bei $\delta$-27.0 die den $\mathrm{SiMe}_{3}$-Gruppen an den Ethinyl-Kohlenstoffatomen zugeordnet werden können.

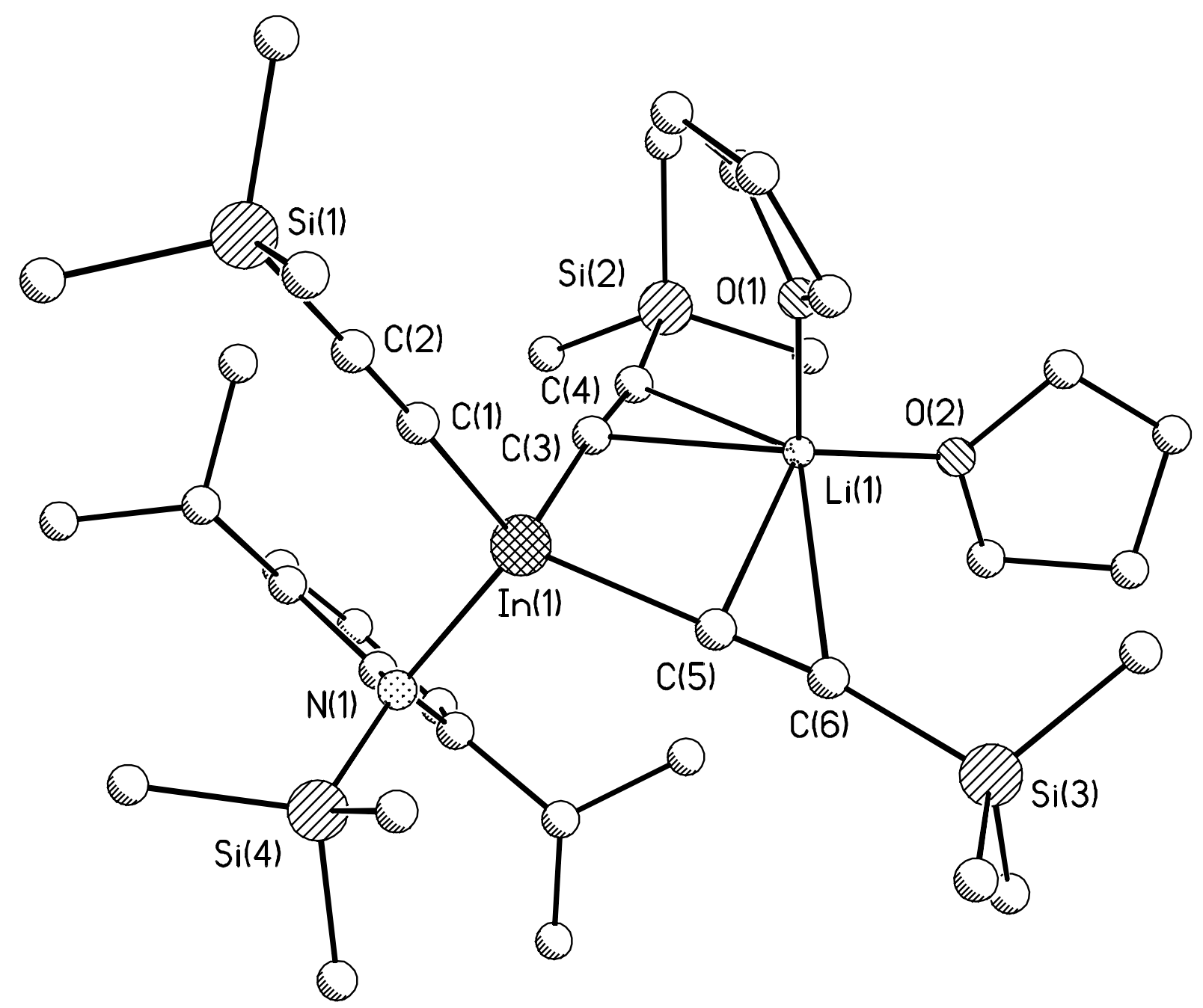

Abb. 9: Molekulare Struktur der Verbindung 11 im Kristall mit THF als koordinierendes Lösungsmittel. (Exakte Daten sind aufgrund eines systematischen Messfehlers nicht erhältlich, dennoch ist die Struktur gesichert) 
In Verbindung 11 koordiniert das Lithium-Kation (im Gegensatz zu Verbindung 10) an die Ethinyl-Gruppen, wenn das Lösungsmittel THF eingesetzt wird.

Offensichtlich ist wie in Verbindung 7 die Koordination des Lithium-Kations an die EthinylGruppen energetisch bevorzugt im Vergleich zu einer Solvatisierung des Lithiums durch die THF-Moleküle.

\subsection{Darstellung von Metall-Diethinyl-Halogen-Verbindungen der 13 . Gruppe des Periodensystems}

\subsubsection{Einleitung}

Trotz ihres großen synthetischen Nutzens in der organischen Chemie ${ }^{[14,65,66,67,68]}$ sind nur wenige Metall-Ethinyl-Verbindungen der 13. Gruppe des Periodensystems des Typs $\mathrm{R}_{\mathrm{n}} \mathrm{M}(\mathrm{C} \equiv \mathrm{CR})_{3-\mathrm{n}} \quad(\mathrm{M}=\mathrm{Al}, \mathrm{Ga}, \mathrm{In} ; \mathrm{R}=\mathrm{Rest})$ bekannt und meist nicht strukturell untersucht worden. Dies gilt ganz besonders für den Typ $\mathrm{RM}(\mathrm{C} \equiv \mathrm{CR})_{2}$, bei dem nur sehr wenige Beispiele $\mathrm{zu}$ finden sind $\left(\mathrm{Al}=3,{ }^{[12,14]} \mathrm{Ga}=0, \mathrm{In}=1^{[63]}\right)$.

Die Herstellung von Verbindungen des Typs $\mathrm{RM}(\mathrm{C} \equiv \mathrm{CR})_{2}$ erfolgte aus den entsprechenden Metallchloriden- bzw. im Fall des Aluminiums auch den -hydriden, deren Herstellung wiederum eine Addition von $\mathrm{MCl}_{3}$ an das entsprechende Lithiumsalz des Liganden voraus ging. Bei den Liganden handelte es sich ausschließlich um chelatisierende Systeme mit Stickstoffbasen. ${ }^{[12,14,63]}$

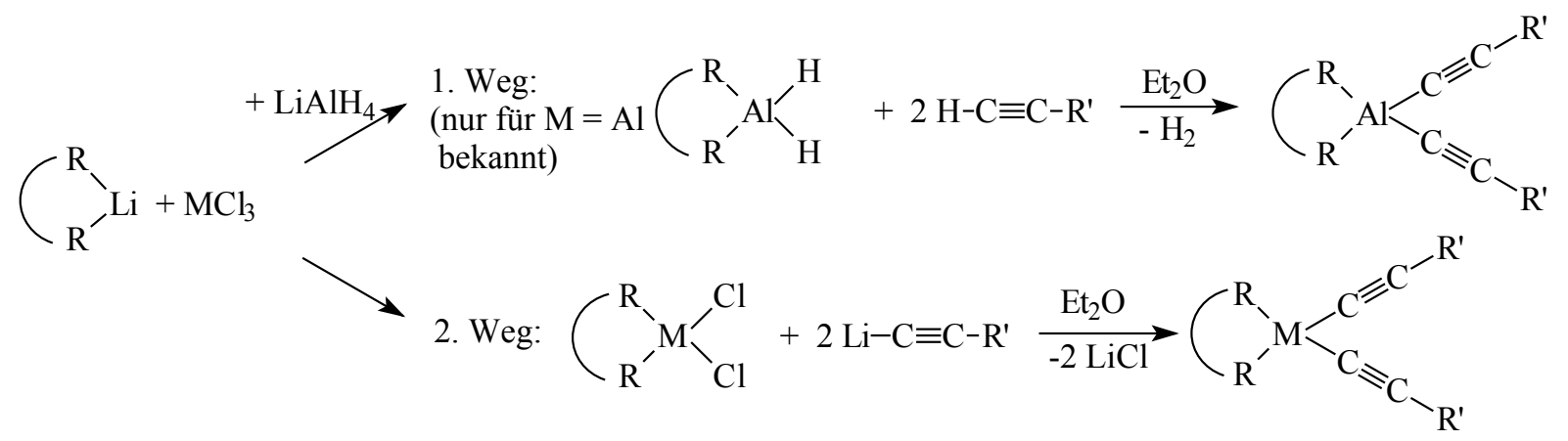

Schema 25: Bekannte Darstellungen von Diethinyl-Aluminium-Verbindungen. ${ }^{[14,63]}$ ( $\mathrm{R}=$ chelatisierender Ligand; $\mathrm{R}^{\prime}=\mathrm{Ph}, \mathrm{SiMe}_{3}$ ) 


\subsubsection{Darstellung von $\mathrm{Br}-\mathrm{Al}(\mathrm{C} \equiv \mathrm{C} t \mathrm{Bu})_{2} \cdot 2$ THF (12)}

$\mathrm{Zu}$ einer Lösung aus $\mathrm{AlBr}_{3}$ in THF wurden bei $-78{ }^{\circ} \mathrm{C}$ zwei Äquivalente $\mathrm{LiC} \equiv \mathrm{C} t \mathrm{Bu}$ gegeben, über Nacht gerührt und anschließend das Lösungsmittel im Vakuum entfernt. Das Gemisch wurde mit Hexan extrahiert. Man erhielt die erste beschriebene Aluminium-HalogenDiethinyl-Verbindung $\mathrm{Br}-\mathrm{Al}(\mathrm{C} \equiv \mathrm{C} t \mathrm{Bu})_{2} \cdot 2 \mathrm{THF}$ (12).

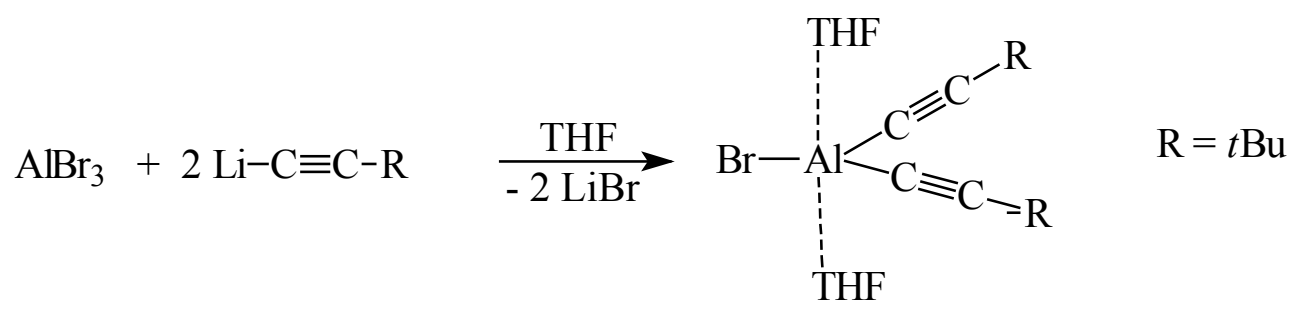

12

Schema 27: Darstellung der ersten Halogen-Aluminium-Diethinyl-Verbindung $\mathrm{Br}-\mathrm{Al}(\mathrm{C} \equiv \mathrm{C} t \mathrm{Bu})_{2} \cdot 2 \mathrm{THF}(\mathbf{1 2})$.

Die Protonen der $t$ Bu-Gruppe erzeugen im ${ }^{1} \mathrm{H}-\mathrm{NMR}-$ Spektrum ein Singulett bei $\delta 1.22$, während die THF-Protonen als Multipletts bei $\delta 1.25$ und $3.78 \mathrm{zu}$ sehen sind. Das ${ }^{13} \mathrm{C}-\mathrm{NMR}$ Spektrum zeigt eine breite Resonanz bei $\delta 67.9$, die den an das Aluminium gebundenen Alkinyl-Kohlenstoffatomen zuzuordnen ist. Die Breite der Resonanz liegt an dem QuadrupolEffekt des Aluminiums (vgl. Kapitel 4.1.1.). Die in $\beta$-Position zum Aluminium gebundenen Kohlenstoffatome erzeugen eine Resonanz bei $\delta$ 92.6. Die übrigen Resonanzen können den ${ }^{13} \mathrm{C}$-Atomen des $t \mathrm{Bu}$-Restes zugeordnet werden. Aufgrund des niedrigen Zersetzungspunkts $\left(145^{\circ} \mathrm{C}\right)$ und des niedrigen Dampfdruckes konnte eine Massenspektrometrie weder mit der EI- der ESI- noch mit der FAB- Ionisierungsmethode durchgeführt werden. Dieses Problem ist bei neutralen Metall-Ethinyl-Verbindungen bekannt und trat mit einer Ausnahme $\left(\mathrm{Ga}(\mathrm{C} \equiv \mathrm{Ct} \mathrm{Bu})_{3}\right)$ durchgehend auf. ${ }^{[58]}$

Die bei der Elementaranalyse gefundenen Werte bestätigen die Zusammensetzung $\mathrm{C}_{20} \mathrm{H}_{34} \mathrm{AlBrO}_{2}$. 


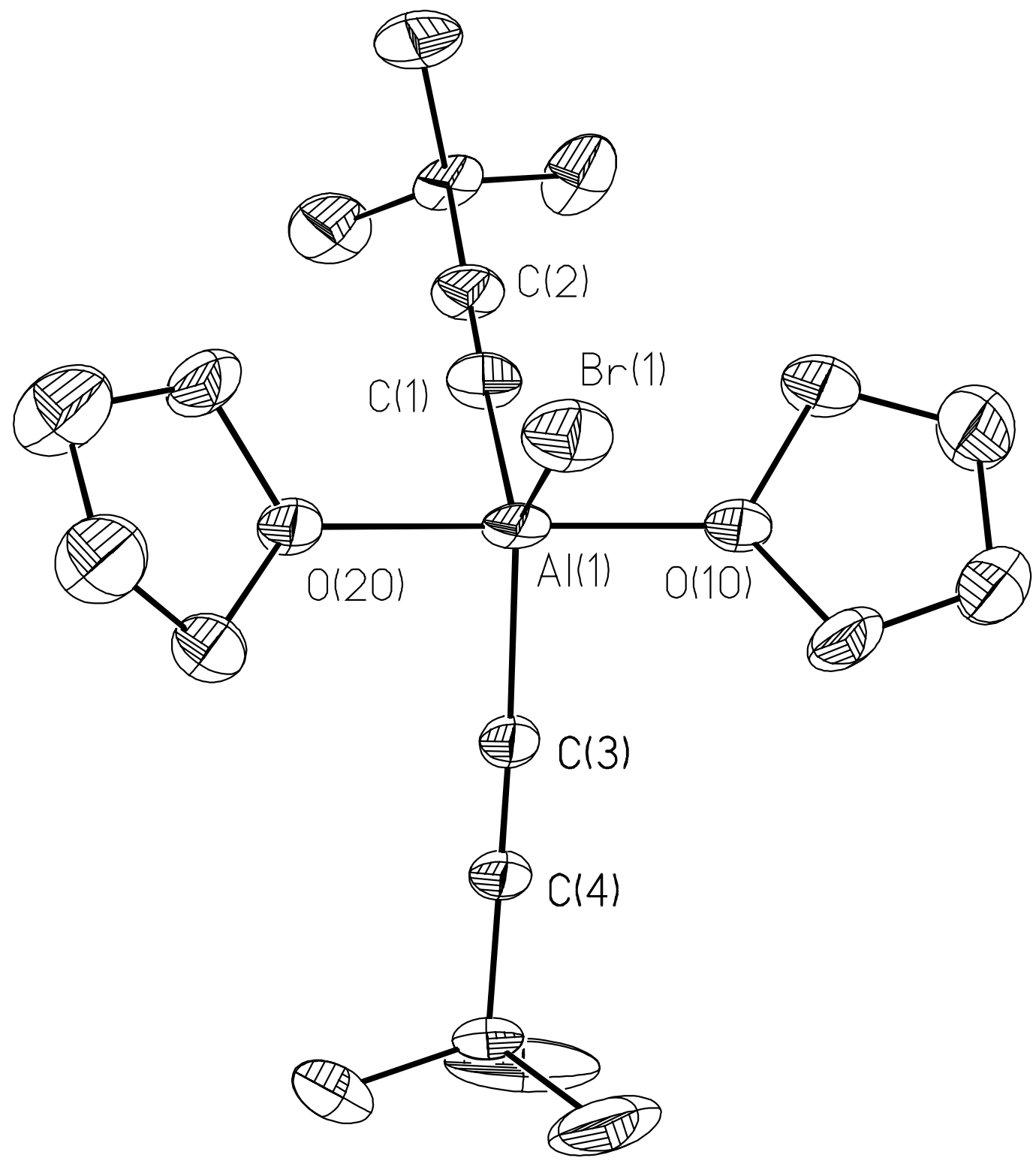

Abb. 10: Struktur von 12 im Kristall.

Ausgewählte Bindungslängen $(\AA)$ und Winkel $\left(^{\circ}\right)$ für 12.

$\begin{array}{lllr}\mathrm{Al}(1)-\mathrm{C}(1) & 1.937(7) & \mathrm{C}(1)-\mathrm{Al}(1)-\mathrm{O}(20) & 89.7(3) \\ \mathrm{Al}(1)-\mathrm{C}(3) & 1.934(7) & \mathrm{C}(1)-\mathrm{Al}(1)-\mathrm{O}(10) & 90.7(3) \\ \mathrm{Al}(1)-\mathrm{O}(20) & 2.018(5) & \mathrm{O}(20)-\mathrm{Al}(1)-\mathrm{O}(10) & 179.5(2) \\ \mathrm{Al}(1)-\mathrm{O}(10) & 2.024(5) & \mathrm{C}(1)-\mathrm{Al}(1)-\mathrm{Br}(1) & 118.1(2) \\ \mathrm{Al}(1)-\mathrm{Br}(1) & 2.353(2) & \mathrm{O}(20)-\mathrm{Al}(1)-\mathrm{Br}(1) & 89.47(15) \\ \mathrm{C}(1)-\mathrm{C}(2) & 1.190(10) & \mathrm{O}(10)-\mathrm{Al}(1)-\mathrm{Br}(1) & 90.17(16) \\ \mathrm{C}(3)-\mathrm{C}(4) & 1.205(10) & \mathrm{C}(2)-\mathrm{C}(1)-\mathrm{Al}(1) & 177.1(7) \\ & & \mathrm{C}(3)-\mathrm{Al}(1)-\mathrm{C}(1) & 125.9(3)\end{array}$


Die Koordinationszahl fünf am Aluminium bei 12 kann durch den elektronenziehenden Effekt (-I-Effekt) der Liganden erklärt werden, durch den das Aluminium-Zentralatom so "Lewissauer" wird, dass es von zwei starken Lewis-Basen (THF) koordiniert wird. ${ }^{[25]}$ Das Molekül hat die Struktur einer trigonalen Bipyramide. In der trigonal-planaren Ebene liegen die zwei an das Aluminiumatom gebundenen $t \mathrm{BuC} \equiv \mathrm{C}$-Liganden sowie das Bromatom. Der C-Al-CWinkel beträgt $125.9^{\circ}$, was für eine ähnliche Abstoßung der Ethinyl-Liganden untereinander wie mit dem Bromatom spricht. Senkrecht zu dieser Ebene stehen die beiden THF-Moleküle $\left(89.47^{\circ}\right.$ und $\left.90.17^{\circ}\right)$. Der Al-O-Abstand (2.018 und $2.024 \AA$ ) ist mit literaturbekannten Al$\mathrm{O}_{\mathrm{THF}}-\mathrm{Abständen} \mathrm{(z.B.} \mathrm{AlCl}_{3} \cdot 2 \mathrm{THF}=1.990 \AA \AA$ ) vergleichbar. ${ }^{[69]}$

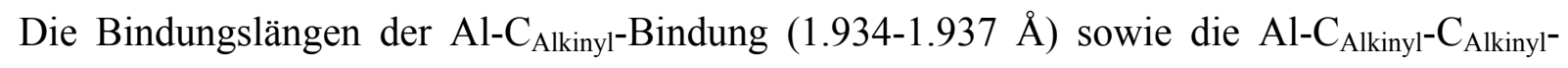
Bindungswinkel $\left(177.1^{\circ}\right.$ ) liegen im Bereich der vorgestellten Verbindungen 4, 5, 6 und 7 (Al-

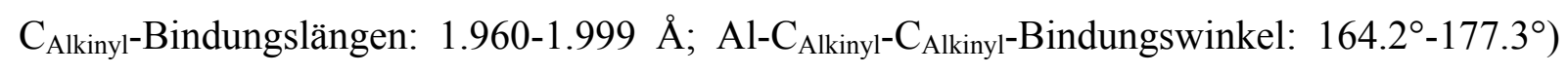
sowie anderer literaturbekannten Aluminium-Diethinyl-Verbindungen ${ }^{[13,}{ }^{14]}$ wie z.B. $\left[(\mathrm{PhC} \equiv \mathrm{C})_{2} \mathrm{Al}\left(t \mathrm{Bu}_{2} \mathrm{pz}\right)_{2} \mathrm{Al}(\mathrm{C} \equiv \mathrm{CPh})_{2}\right]\left(\mathrm{Al}-\mathrm{C}_{\text {Alkinyl-Bindungslängen: }}\right.$ 1.913-1.929 $\AA$; Al- $\mathrm{C}_{\text {Alkinyl- }}$

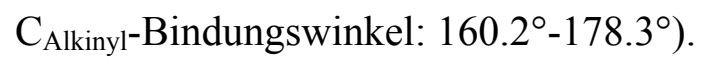

Die Darstellung von $\mathrm{Br}-\mathrm{Al}(\mathrm{C} \equiv \mathrm{C} t \mathrm{Bu})_{2} \cdot 2 \mathrm{THF}$ (12) zeigt, dass es einen einfachen Weg zur Darstellung von Aluminiumverbindungen mit zwei terminalen Ethinyl-Gruppen gibt. Es eignen sich nicht alle Substituenten gleichermaßen für diese Reaktion. Die Reaktion von Aluminiumtribromid mit zwei Äquivalenten Li-C $\equiv \mathrm{C}-\mathrm{Ph}$ in $\mathrm{THF}$ ergibt einen zähen Rückstand, der erst mit Dioxan versetzt fest wird. Das ${ }^{13} \mathrm{C}-\mathrm{NMR}-$ Spektrum zeigt, dass es sich bei der Reaktion von $\mathrm{AlBr}_{3}$ mit $\mathrm{Li}-\mathrm{C} \equiv \mathrm{C}-\mathrm{Ph}$ nicht um ein einheitliches Produkt handelt sondern um ein Produktgemisch.

\subsubsection{Darstellung von $\mathrm{Cl}-\mathrm{Ga}(\mathrm{C} \equiv \mathrm{C} t \mathrm{Bu})_{2} \cdot \mathrm{THF}(13)$}

$\mathrm{Zu}$ einer Lösung von $\mathrm{GaCl}_{3}$ in THF wurden bei $-78{ }^{\circ} \mathrm{C}$ zwei Äquivalente $\mathrm{LiC} \equiv \mathrm{C} t \mathrm{Bu}$ hinzugegeben und bei Raumtemperatur über Nacht gerührt. Das Lösungsmittel wurde im Vakuum entfernt und das Produkt mit Hexan extrahiert. Nach Trocknen im Vakuum konnte $\mathrm{Cl}-\mathrm{Ga}(\mathrm{C} \equiv \mathrm{C} t \mathrm{Bu})_{2} \cdot \mathrm{THF}(\mathbf{1 3})$ als farblose Verbindung erhalten werden. Es handelt sich hierbei um die erste Verbindung des Galliums mit zwei terminalen Ethinyl-Gruppen. 


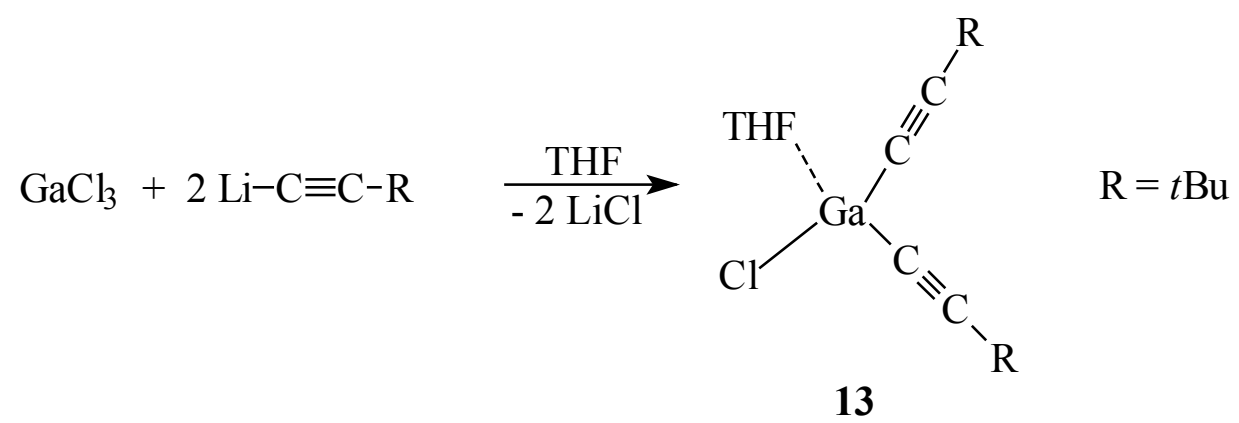

Schema 28: Darstellung von $\mathrm{Cl}-\mathrm{Ga}(\mathrm{C} \equiv \mathrm{C} t \mathrm{Bu})_{2} \cdot \mathrm{THF}(\mathbf{1 3})$

Ähnlich wie in Verbindung 12 zeigt das ${ }^{1} \mathrm{H}-\mathrm{NMR}-$ Spektrum ein Singulett bei $\delta 1.17$, während die THF-Protonen Multipletts bei $\delta 1.08$ und 3.81 erzeugen. Im ${ }^{13} \mathrm{C}-\mathrm{NMR}-$ Spektrum sieht man eine im Vergleich mit der entsprechenden Aluminium-Verbindung 12 deutlich Tieffeld verschobene breite Resonanz bei $\delta 83.7$ der in $\alpha$-Position zum Gallium gebundenen Kohlenstoffatome. Das breite Erscheinungsbild der Resonanz liegt auch hier an dem Quadrupol-Effekt des Galliums. Die in $\beta$-Position zum Gallium gebundenen AlkinylKohlenstoffatome zeigen eine Resonanz bei $\delta$ 116.4. Die übrigen Signale können dem $t \mathrm{Bu}-$ Rest zugeordnet werden. Die Elementaranalyse bestätigt die gefundene Zusammensetzung $\mathrm{C}_{16} \mathrm{H}_{26} \mathrm{ClGaO}$.

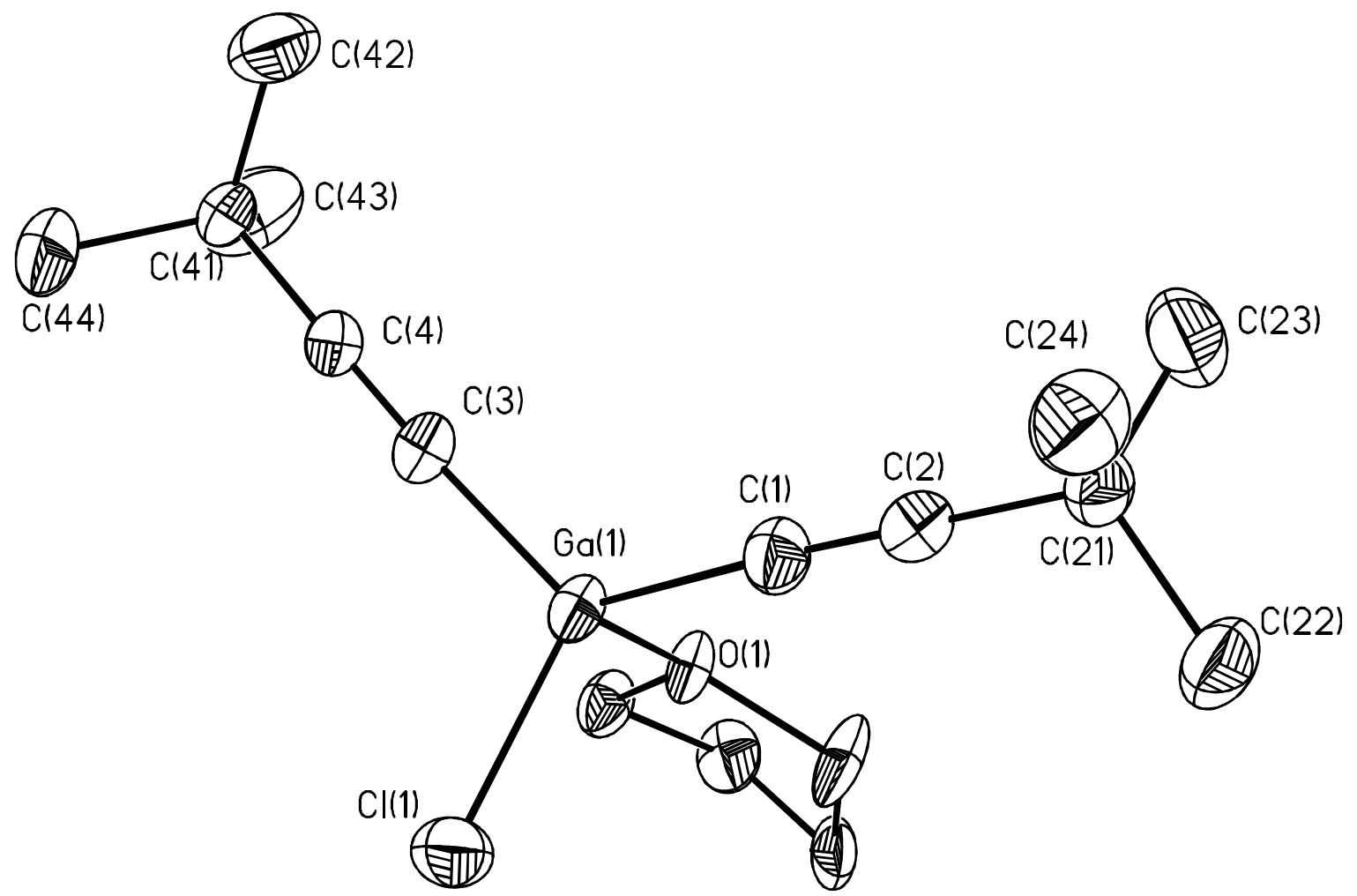

Abb. 11: Struktur von $\mathrm{Cl}-\mathrm{Ga}(\mathrm{C} \equiv \mathrm{C} t \mathrm{Bu})_{2} \cdot \mathrm{THF}(\mathbf{1 3})$ im Kristall. 
Ausgewählte Bindungslängen $(\AA)$ und Winkel $\left(^{\circ}\right)$ für $\mathrm{Cl}-\mathrm{Ga}(\mathrm{C} \equiv \mathrm{C} t \mathrm{Bu})_{2} \cdot \mathrm{THF}$ (13).

$\begin{array}{llll}\mathrm{Ga}(1)-\mathrm{C}(3) & 1.908(2) & \mathrm{C}(3)-\mathrm{Ga}(1)-\mathrm{O}(1) & 100.5(3) \\ \mathrm{Ga}(1)-\mathrm{C}(1) & 1.911(2) & \mathrm{C}(1)-\mathrm{Ga}(1)-\mathrm{O}(1) & 99.2(3) \\ \mathrm{Ga}(1)-\mathrm{O}(1) & 2.006(10) & \mathrm{C}(3)-\mathrm{Ga}(1)-\mathrm{Cl}(1) & 113.08(7) \\ \mathrm{Ga}(1)-\mathrm{Cl}(1) & 2.1953(10) & \mathrm{C}(1)-\mathrm{Ga}(1)-\mathrm{Cl}(1) & 111.42(8) \\ \mathrm{C}(1)-\mathrm{C}(2) & 1.196(3) & \mathrm{O}(1)-\mathrm{Ga}(1)-\mathrm{Cl}(1) & 103.8(3) \\ \mathrm{C}(3)-\mathrm{C}(4) & 1.198(3) & \mathrm{C}(2)-\mathrm{C}(1)-\mathrm{Ga}(1) & 175.5(2) \\ \mathrm{C}(3)-\mathrm{Ga}(1)-\mathrm{C}(1) & 124.61(10) & \mathrm{C}(4)-\mathrm{C}(3)-\mathrm{Ga}(1) & 175.3(2)\end{array}$

Das Galliumatom ist im Gegensatz zu 12 in diesem Fall nur vierfach koordiniert. Dies ist in Galliumorganylen die häufigste Koordinationszahl. ${ }^{[62]}$

Wie bei anderen Produkten in denen das Galliumatom vierfach koordiniert ist, wovon eine Bindungsstelle von einer Lewis-Base besetzt ist, verkleinert sich der Winkel zwischen der Lewis-Base und den kovalent gebundenen Liganden. ${ }^{[70]}$ Dies kann mit einer Annäherung an eine sp $^{2}$-Hybridisierung am Galliumatom erklärt werden, bei der das freie Elektronenpaar des THF-Moleküls mit einem Orbital des Galliums mit hohem p-Charakter wechselwirkt. ${ }^{[71]}$

Dem bei einer Gallium $\mathrm{sp}^{2}$-Hybridisierung erwarteten Winkel zwischen dem p-Orbital und den $\mathrm{sp}^{2}$-Hybridorbitalen von $90^{\circ}$ kommen die gefundenen Werte nahe. So beträgt z. B. der $\mathrm{C}(1)-\mathrm{Ga}(1)-\mathrm{O}(1)-$ Winkel $99.2^{\circ}\left(\left(\mathrm{C}(3)-\mathrm{Ga}(1)-\mathrm{O}(1) 100.5^{\circ}\right.\right.$; O(1)-Ga(1)-Cl(1) 103.8 $)$. Da eine kovalentere Bindung immer energetisch niedrigere Orbitale mit einem höheren s-Charakter nutzen wird (so wie ein $\mathrm{sp}^{2}$-Hybridorbital) und die schwächeren koordinativen Bindungen notwendigerweise mit den energetisch höher liegenden Orbitalen mit p-Charakter (Bent's Regel) wechselwirken, ${ }^{[72]}$ ist der Winkel zwischen den koordinierenden Basen und den kovalent gebundenen Liganden immer kleiner als der zwischen den kovalent gebundenen Liganden. Triebkraft ist die größere sterische Abstoßung der kovalenten Bindungspartner, die dichter am Metall sind.

Dies ist hier der Fall, denn alle Winkel zwischen dem kovalent gebundenen Chlor- und den Ethinyl-Liganden sind größer als der Tetraeder-Winkel von $109^{\circ}$ (z. B. C(3)-Ga(1)-C(1) $124.6^{\circ}\left(\mathrm{C}(3)-\mathrm{Ga}(1)-\mathrm{Cl}(1) 113.1^{\circ} ; \mathrm{C}(1)-\mathrm{Ga}(1)-\mathrm{Cl}(1) 111.4^{\circ}\right)$.

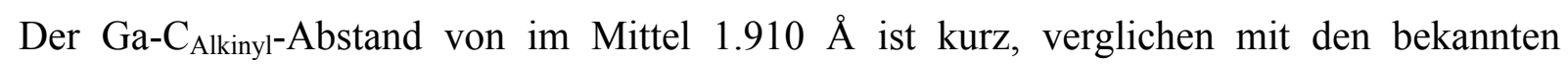
Bindungslängen (vgl. Tab. 3). ${ }^{[57,58,59,60]}$ 


\subsubsection{Darstellung von $\mathrm{Cl}-\mathrm{In}(\mathrm{C} \equiv \mathrm{C} t \mathrm{Bu})_{2} \cdot 2 \mathrm{THF}$ (14)}

Eine Lösung aus $\mathrm{InCl}_{3}$ in THF wurde bei $-78^{\circ} \mathrm{C}$ mit zwei Äquivalenten $\mathrm{LiC} \equiv \mathrm{C} t \mathrm{Bu}$ versetzt und über Nacht bei Raumtemperatur gerührt. Anschließend wurde das Lösungsmittel im Vakuum entfernt und das Produkt mit Hexan extrahiert. Man erhielt 14 als einen farblosen Feststoff. Dabei handelt es sich um die erste Indium-Halogen-Diethinyl-Verbindung. Es ist die zweite bekannte Verbindung des Indiums ${ }^{[63]}$ mit zwei terminalen Ethinyl-Gruppen. Beide Verbindungen stammen aus unserem Arbeitskreis.

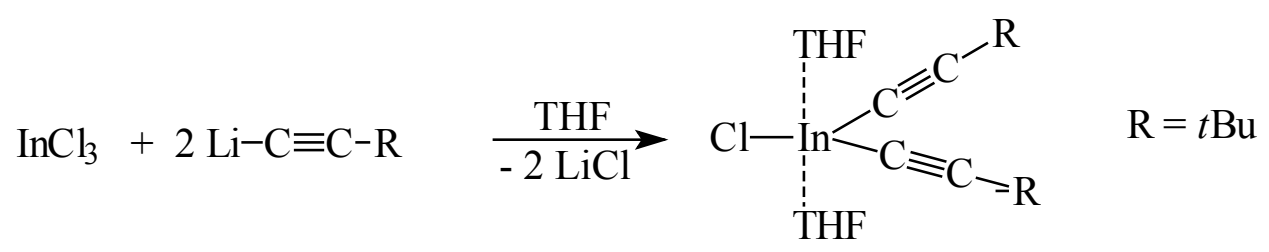

14

Schema 29: Darstellung von 14.

Das ${ }^{1} \mathrm{H}-\mathrm{NMR}-$ Spektrum ähnelt den Verbindungen 12 und 13. Es zeigt ein Singulett bei $\delta 1.24$, während die THF-Protonen Multipletts bei $\delta 1.30$ und 3.84 erzeugen. Das ${ }^{13} \mathrm{C}-\mathrm{NMR}$ Spektrum zeigt auch hier eine breite Resonanz bei $\delta 88.3$, welche dem an das Indium gebundenen Alkinyl-Kohlenstoffatom zuzuordnen ist. Die Verschiebung ist mit der GalliumVerbindung 13 zu vergleichen, ist aber leicht hochfeldverschoben. Dies kann mit der höheren Elektronendichte am Kohlenstoffatom (in $\alpha$-Position zum Indium) erklärt werden, da mehr Basen an das Indium als an das Gallium koordiniert sind. Die Breite des Signals liegt auch hier an dem Quadrupol-Effekt des Indiums. Die in $\beta$-Position zum Indium gebundenen Ethinyl-Kohlenstoffatome erzeugen eine Resonanz bei $\delta$ 118.2. Die übrigen Signale können dem $t$ Bu-Rest zugeordnet werden. Die Elementaranalyse bestätigt die gefundene Zusammensetzung $\mathrm{C}_{20} \mathrm{H}_{34} \mathrm{ClInO}_{2}$. 


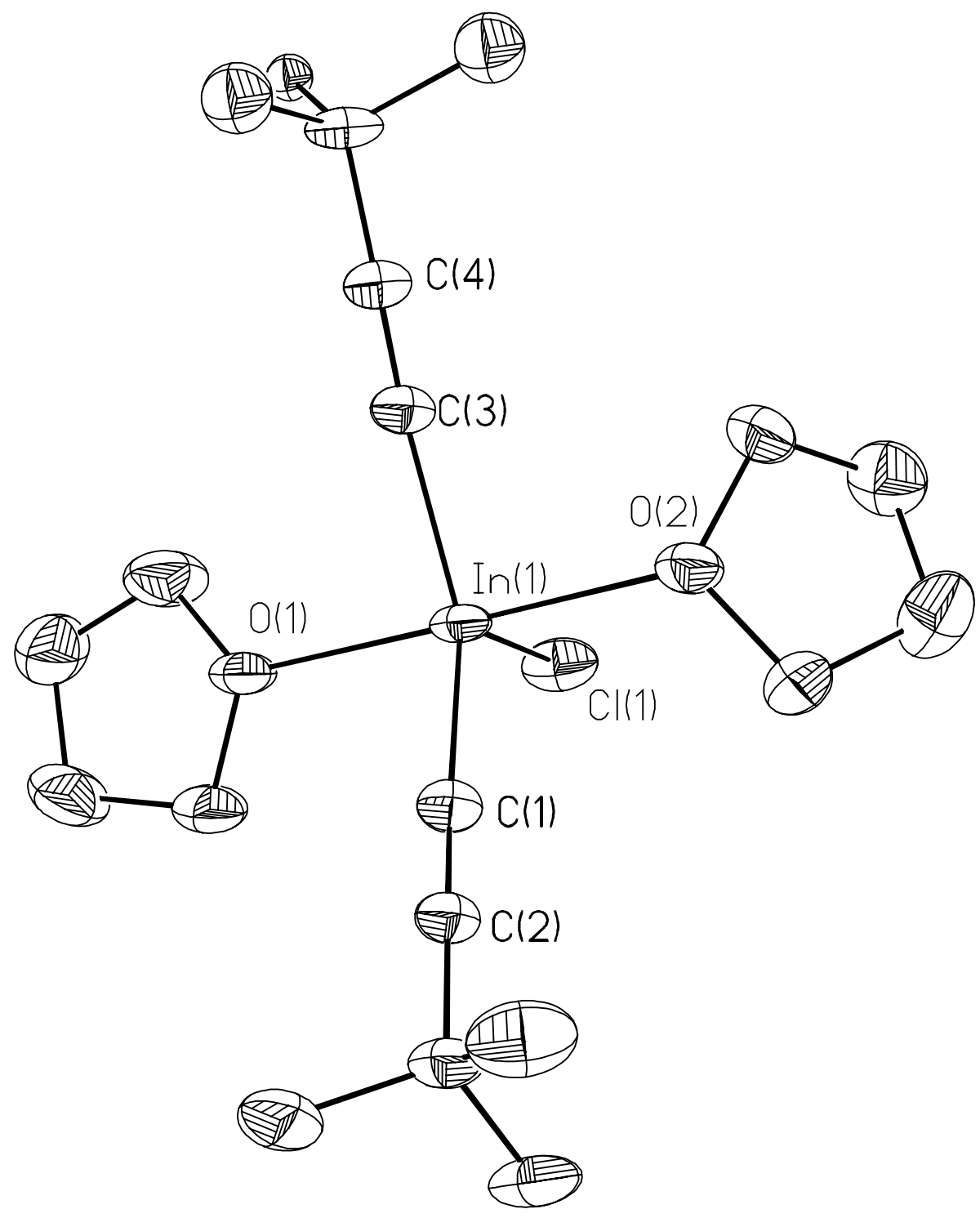

Abb. 12: Struktur von 14 im Kristall.

Ausgewählte Bindungslängen $(\AA)$ und Winkel $\left(^{\circ}\right)$ für 14.

$\begin{array}{llll}\mathrm{Cl}(1)-\operatorname{In}(1) & 2.387(1) & \mathrm{C}(3)-\operatorname{In}(1)-\mathrm{O}(2) & 88.19(12) \\ \mathrm{In}(1)-\mathrm{C}(3) & 2.101(4) & \mathrm{C}(1)-\operatorname{In}(1)-\mathrm{O}(2) & 89.05(12) \\ \mathrm{In}(1)-\mathrm{C}(1) & 2.104(4) & \mathrm{O}(1)-\operatorname{In}(1)-\mathrm{O}(2) & 178.99(10) \\ \mathrm{In}(1)-\mathrm{O}(1) & 2.330(3) & \mathrm{C}(3)-\operatorname{In}(1)-\mathrm{Cl}(1) & 114.03(12) \\ \mathrm{In}(1)-\mathrm{O}(2) & 2.351(3) & \mathrm{C}(1)-\operatorname{In}(1)-\mathrm{Cl}(1) & 115.27(12) \\ \mathrm{C}(1)-\mathrm{C}(2) & 1.196(6) & \mathrm{O}(1)-\operatorname{In}(1)-\mathrm{Cl}(1) & 90.85(9) \\ \mathrm{C}(3)-\mathrm{C}(4) & 1.197(5) & \mathrm{O}(2)-\operatorname{In}(1)-\mathrm{Cl}(1) & 90.08(8) \\ \mathrm{C}(3)-\operatorname{In}(1)-\mathrm{C}(1) & 130.61(17) & \mathrm{C}(2)-\mathrm{C}(1)-\operatorname{In}(1) & 177.6(4) \\ \mathrm{C}(3)-\operatorname{In}(1)-\mathrm{O}(1) & 91.07(12) & \mathrm{C}(4)-\mathrm{C}(3)-\operatorname{In}(1) & 175.9(3) \\ \mathrm{C}(1)-\operatorname{In}(1)-\mathrm{O}(1) & 90.90(12) & & \end{array}$


Wie die homologe Aluminiumverbindung 12 besitzt auch dieses Molekül die Grundstruktur einer trigonalen Bipyramide. In der trigonal-planaren Ebene liegen die zwei an das Indiumatom gebundenen $t \mathrm{BuC} \equiv \mathrm{C}$-Liganden sowie das Chloratom und senkrecht dazu sind die zwei THF-Moleküle angeordnet. Der C(3)-In(1)-C(1)-Winkel beträgt $130.6^{\circ}$ und ist damit größer als der Idealwinkel von $120^{\circ}$, während die Winkel zwischen den Ethinyl-Liganden und dem Chloratom kleiner als $120^{\circ}$ sind $\left(114.0^{\circ}\right.$ und $\left.115.3^{\circ}\right)$. Der Winkel der Liganden in der trigonal-planaren Ebene zu den Sauerstoffatomen der THF-Moleküle weicht nie mehr als $2^{\circ}$ vom Idealwinkel von $90^{\circ} \mathrm{ab}$.

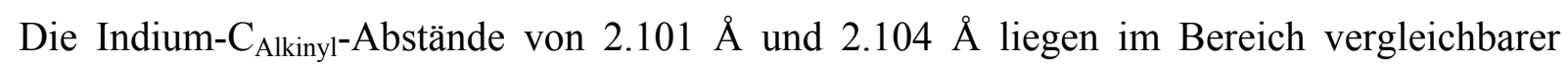
Verbindungen (vgl. Tab. 4) wie z.B. 2.11-2.13 $\AA$ in $\operatorname{In}\left(\mathrm{C} \equiv \mathrm{CCF}_{3}\right)_{3} \cdot 2$ THF. ${ }^{[62]}$

\subsubsection{Reaktionsverhalten der Verbindungen 12, 13 und 14}

Die erfolgreiche Synthese von 12, 13 und 14 ließ eine einfache Derivatisierung mit Metallalkylen zu Monoalkyl-Diethinyl-Metallanen erwarten.

$$
\mathrm{RLi}+\mathrm{X}-\mathrm{M}(\mathrm{C} \equiv \mathrm{CR})_{2} \rightarrow \mathrm{R}-\mathrm{M}(\mathrm{C} \equiv \mathrm{C}-\mathrm{R})_{2}+\mathrm{LiX} \quad(\mathrm{M}=\mathrm{Al}, \mathrm{Ga}, \mathrm{In} ; \mathrm{R}=\mathrm{Rest})
$$

Schema 30: Erwartete einfache Darstellung neuer Metall-Diethinyl-Verbindungen aus dem Lithiumsalz von 2,6-i $\mathrm{Pr}_{2} \mathrm{C}_{6} \mathrm{H}_{3} \mathrm{~N}\left(\mathrm{SiMe}_{3}\right)$.

Es zeigte zeigt sich jedoch, dass die Reaktion von 2,6-i $\mathrm{Pr}_{2} \mathrm{C}_{6} \mathrm{H}_{3} \mathrm{~N}\left(\mathrm{SiMe}_{3}\right) \mathrm{Li}$ mit 12, 13 oder 14 nicht zu dem erwarteten Produkt führte, sondern zu Produktgemischen. Bei der Reaktion der Galliumverbindung $\mathrm{Cl}-\mathrm{Ga}(\mathrm{C} \equiv \mathrm{C} t \mathrm{Bu})_{2} \cdot$ THF mit 2,6-i $\mathrm{Pr}_{2} \mathrm{C}_{6} \mathrm{H}_{3} \mathrm{~N}\left(\mathrm{SiMe}_{3}\right)$ Li fielen im Tiefkühlschrank neben einem amorphen Feststoff farblose Kristalle aus, die durch eine Röntgenstrukturanalyse als $\left[\mathrm{Li} \cdot 2 \mathrm{THF}\left(\mathrm{R}-\mathrm{Ga}(\mathrm{C} \equiv \mathrm{C} t \mathrm{Bu})_{3}\right)\right](\mathbf{1 5})$ identifiziert werden konnten.

$$
\text { 2,6-i } \mathrm{Pr}_{2} \mathrm{C}_{6} \mathrm{H}_{3} \mathrm{~N}\left(\mathrm{SiMe}_{3}\right) \mathrm{Li}+\mathbf{1 3} \rightarrow \mathbf{1 5}+\text { Produktgemisch }
$$

Schema 31: Reaktion von $\mathrm{Cl}-\mathrm{Ga}(\mathrm{C} \equiv \mathrm{C} t \mathrm{Bu})_{2} \cdot \mathrm{THF}(\mathbf{1 3})$ mit 2,6-i $\mathrm{Pr}_{2} \mathrm{C}_{6} \mathrm{H}_{3} \mathrm{~N}\left(\mathrm{SiMe}_{3}\right) \mathrm{Li}$. 


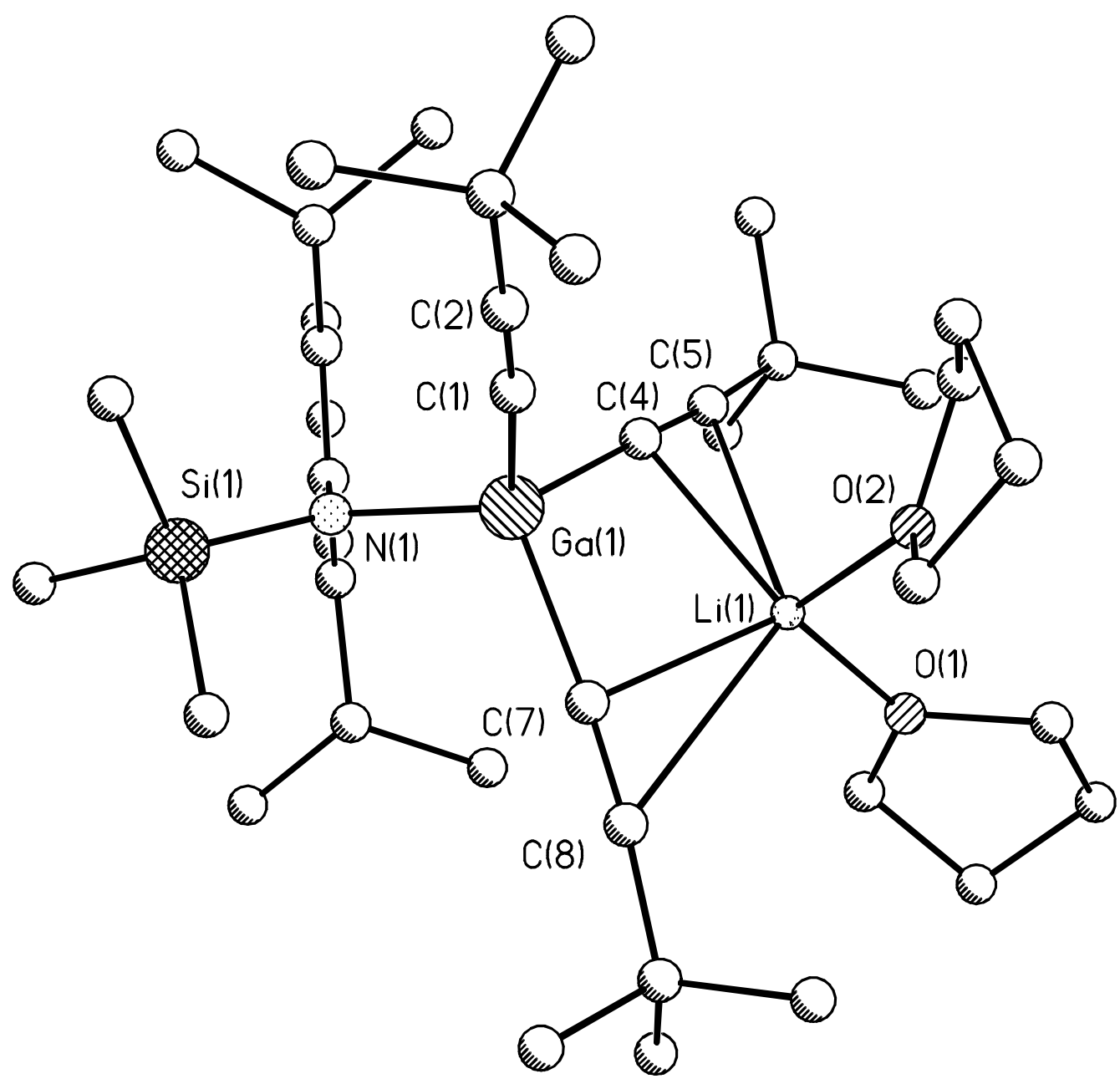

Abb. 13: Struktur von 15 im Kristall.

Ausgewählte Bindungslängen $(\AA)$ und Winkel $\left(^{\circ}\right)$ für 15.

$\begin{array}{llll}\mathrm{N}(1)-\mathrm{Ga}(1) & 1.903(2) & \mathrm{C}(1)-\mathrm{Li}-\mathrm{C}(2) & 26.08(8) \\ \mathrm{Li}-\mathrm{C}(1) & 2.316(5) & \mathrm{C}(7)-\mathrm{Li}-\mathrm{C}(8) & 25.29(9) \\ \mathrm{Li}-\mathrm{C}(2) & 2.720(5) & \mathrm{N}(1)-\mathrm{Ga}(1)-\mathrm{C}(13) & 111.41(8) \\ \mathrm{Li}-\mathrm{C}(7) & 2.321(4) & \mathrm{N}(1)-\mathrm{Ga}(1)-\mathrm{C}(1) & 108.36(9) \\ \mathrm{Li}-\mathrm{C}(8) & 2.780(5) & \mathrm{C}(13)-\mathrm{Ga}(1)-\mathrm{C}(1) & 113.42(9) \\ \mathrm{Ga}(1)-\mathrm{C}(1) & 1.979(2) & \mathrm{N}(1)-\mathrm{Ga}(1)-\mathrm{C}(7) & 111.77(9) \\ \mathrm{Ga}(1)-\mathrm{C}(7) & 1.991(3) & \mathrm{C}(13)-\mathrm{Ga}(1)-\mathrm{C}(7) & 109.25(10) \\ \mathrm{Ga}(1)-\mathrm{C}(13) & 1.956(2) & \mathrm{C}(1)-\mathrm{Ga}(1)-\mathrm{C}(7) & 102.33(9) \\ \mathrm{C}(1)-\mathrm{C}(2) & 1.203(3) & \mathrm{C}(2)-\mathrm{C}(1)-\mathrm{Ga}(1) & 174.7(2) \\ \mathrm{C}(7)-\mathrm{C}(8) & 1.203(3) & \mathrm{C}(8)-\mathrm{C}(7)-\mathrm{Ga}(1) & 176.2(2) \\ \mathrm{C}(14)-\mathrm{C}(13) & 1.198(3) & \mathrm{C}(14)-\mathrm{C}(13)-\mathrm{Ga}(1) & 175.4(2)\end{array}$


Im Gegensatz zu der vergleichbaren Verbindung 9, bei der das Lithium-Kation von Dioxan koordiniert ist, wird hier das Lithium-Kation von THF-Molekülen koordiniert.

Wie schon beim Vergleich der Verbindungen 10 und 11 festgestellt wurde, koordiniert das Lithium-Kation beim Einsatz von THF als Lösungsmittel an die zwei Ethinyl-Gruppen und zwei THF-Moleküle

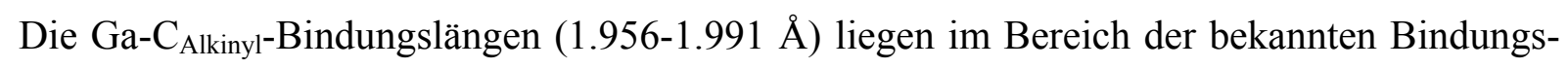
längen (vgl. Tab. 3). Im Vergleich mit 9 weichen die Ga-C $\equiv \mathrm{C}$-Bindungswinkel in $\mathbf{1 5}$ nicht so stark von $180^{\circ}$ ab $\left(9: 169.9^{\circ}-173.7^{\circ} ; 15: 174.7^{\circ}-176.2^{\circ}\right)$. Die Abstände des Lithium-Kations in $15 \mathrm{zu}$ den in $\alpha$-Position zum Aluminium gebundenen Ethinyl-Kohlenstoffatomen (2.316$2.321 \AA$ ) sowie $\mathrm{zu}$ den in $\beta$-Position zum Aluminiumatom gebundenen AlkinylKohlenstoffatomen (2.720-2.780 $\AA$ ) können mit den Abständen in 7 (Li-C $\alpha: 2.333$ und $2.343 \AA$; Li-C $\mathrm{C}_{\beta}: 2.734$ und $2.768 \AA$ ) verglichen werden. Da die C $\equiv \mathrm{C}$-Abstände mit 1.198$1.203 \AA$ ähnlich groß wie in 7 sind (1.205-1.215 $\AA$ ), sind somit auch die C-Li-C-Bindungswinkel vergleichbar (15: $25.29^{\circ}$ und 26.08 $;$ 7: $25.44^{\circ}$ und $\left.26.26^{\circ}\right)$.

Anhand dieser Reaktionen von 2,6-i $\mathrm{Pr}_{2} \mathrm{C}_{6} \mathrm{H}_{3} \mathrm{~N}\left(\mathrm{SiMe}_{3}\right) \mathrm{Li}$ mit 13 ist $\mathrm{zu}$ sehen, dass Verbindungen der Form R-M $(\mathrm{C} \equiv \mathrm{C} t \mathrm{Bu})_{2} \cdot \mathrm{n}$ THF nicht stabil sind. So bleibt der Schluss, dass eine Metall-Diethinyl-Verbindung des Typs $\mathrm{R}-\mathrm{M}(\mathrm{C} \equiv \mathrm{CR})_{2}$, bei der $\mathrm{R}$ einen einzähnigen, sterisch anspruchsvollen Stickstoff-Liganden darstellt, nicht über diesen Weg darstellbar ist. So sollte dieser Weg aber bei chelatisierenden Ligandensystemen möglich sein. 


\subsection{Darstellung von Triethinylmetallanen der 13. Gruppe des Periodensystems}

\subsubsection{Einleitung}

Wie in Kapitel 2.5.1 erwähnt, spielen Metall-Triethinyl-Verbindungen der 13. Gruppe des Periodensystems in der organischen Synthese eine große Rolle. ${ }^{[65,66,67,68]}$

Trotzdem sind nur wenige Aluminium-Verbindungen des Typs $\mathrm{Al}(\mathrm{C} \equiv \mathrm{CR})_{3}$ dargestellt worden. ${ }^{[65,66,73]}$ Es ist lediglich die Synthese von zwei Gallium- und Indiumverbindungen dieses Typs beschrieben. ${ }^{[68,73]}$

Eine strukturelle Untersuchung dieser Verbindungsklasse ist jedoch nur im Falle des Indiums $^{[62]}$ und zeitnah in diesem Arbeitskreis im Falle des Aluminiums ${ }^{[74,75]}$ erfolgt. Der Aufbau und die strukturelle Untersuchung dieser Verbindungsklasse ist in dieser Arbeit am Beispiel neuer Verbindungen erfolgt.

\subsubsection{Darstellung von $\mathrm{Al}(\mathrm{C} \equiv \mathrm{C} t \mathrm{Bu})_{3} \cdot \operatorname{Dioxan}(16)$}

$\mathrm{Zu}$ einer Lösung aus $\mathrm{AlBr}_{3}$ in Dioxan wurden bei $-78^{\circ} \mathrm{C}$ drei Äquivalente $\mathrm{LiC} \equiv \mathrm{C} t \mathrm{Bu}$ gegeben und die Mischung über Nacht bei Raumtemperatur rühren gelassen. Anschließend wurde das Gemisch filtriert. Nach Entfernung des Lösungsmittels vom Filtrat wurde $\mathrm{Al}(\mathrm{C} \equiv \mathrm{C} t \mathrm{Bu})_{3}$. Dioxan (16) in guter Ausbeute (82\%) erhalten.

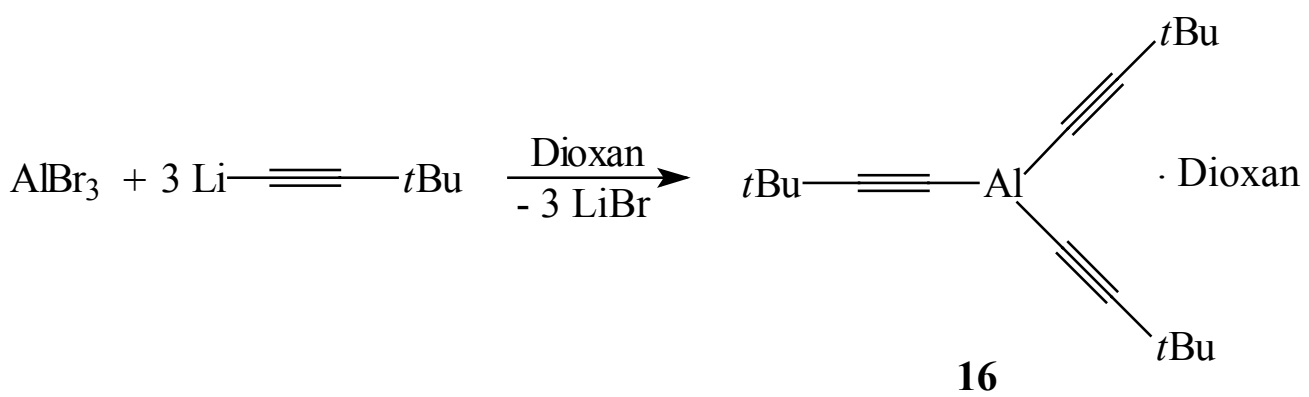

Schema 32: Darstellung von 16.

Das ${ }^{1}$ H-NMR-Spektrum zeigt zwei Singuletts für die Protonen der $t$ Bu-Gruppen $(\delta 1.14)$ und für koordinierendes Dioxan $(\delta 3.56)$, die im Intensitätsverhältnis $27: 8$ zueinander stehen. Im

${ }^{13} \mathrm{C}$-NMR-Spektrum sind die erwarteten Signale für das Dioxan sowie für die $t \mathrm{Bu}-\mathrm{Kohlen}-$ stoffatome und ein breites Signal bei $\delta 94.1$ zu sehen, das den an das Aluminium gebundenen Kohlenstoffatomen zugeordnet werden kann. Es ist aufgrund des Quadrupol-Effekts breit. 
Eine weitere Resonanz bei $\delta 112.6$ kann den in $\beta$-Position zum Aluminium gebundenen Kohlenstoffatomen zugeordnet werden. Die Elementaranalyse bestätigt die gefundene Zusammensetzung $\mathrm{C}_{22} \mathrm{H}_{35} \mathrm{AlO}_{2}$.

\subsubsection{Darstellung von $\mathrm{Ga}(\mathrm{C} \equiv \mathrm{C} t \mathrm{Bu})_{3} \cdot \operatorname{Dioxan}(17)$}

Um 17 zu erhalten, wurden drei Äquivalente $\mathrm{LiC} \equiv \mathrm{C} t \mathrm{Bu}$ in Dioxan gelöst, auf $-78^{\circ} \mathrm{C}$ gekühlt und zu der kalten Lösung ein Äquivalent $\mathrm{GaCl}_{3}$ in Dioxan gegeben. Die Mischung wurde über Nacht bei Raumtemperatur gerührt und nach Filtration und Entfernen des Lösungsmittels vom Filtrat das gewünschte $\mathrm{Ga}(\mathrm{C} \equiv \mathrm{C} t \mathrm{Bu})_{3} \cdot$ Dioxan $(17)$ in sehr guter Ausbeute $(67 \%)$ erhalten.

Für eine Röntgenstrukturanalyse geeignete Einkristalle wurden durch Lagerung einer gesättigten THF-Lösung bei $12{ }^{\circ} \mathrm{C}$ nach 4 Tagen erhalten.

Es handelt sich hierbei um die erste strukturell charakterisierte Galliumverbindung mit drei terminalen Ethinyl-Gruppen.

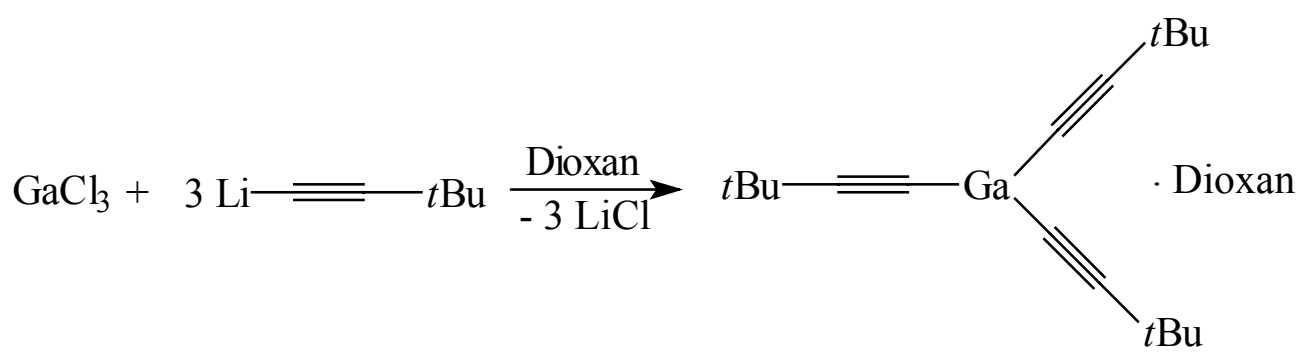

17

Schema 33: Darstellung von 17.

Die NMR-spektroskopische Untersuchung zeigt im ${ }^{1} \mathrm{H}-\mathrm{NMR}-\mathrm{Spektrum}$ zwei Singuletts, eins bei $\delta 1.15$ für die $t$ Bu-Gruppen und eins bei $\delta 3.54$ für das Dioxan, im Intensitätsverhältnis von $27: 8$. Das ${ }^{13} \mathrm{C}$-NMR-Spektrum zeigt die erwarteten Resonanzen für das Dioxan, für die $t$ Bu-Kohlenstoffatome, für die in $\beta$-Position zum Gallium gebundenen AlkinylKohlenstoffatome und ein breites Signal bei $\delta 86.3$, welches den an das Gallium gebundenen Kohlenstoffatomen zugeordnet werden kann. Das Signal hat aufgrund des Quadrupol-Effekts ein breites Erscheinungsbild.

Das Massenspektrum zeigt den Molpeak (312) von 17 ohne das koordinierende Dioxan. Die Elementaranalyse bestätigt die gefundene Zusammensetzung $\mathrm{C}_{22} \mathrm{H}_{35} \mathrm{GaO}_{2}$. 


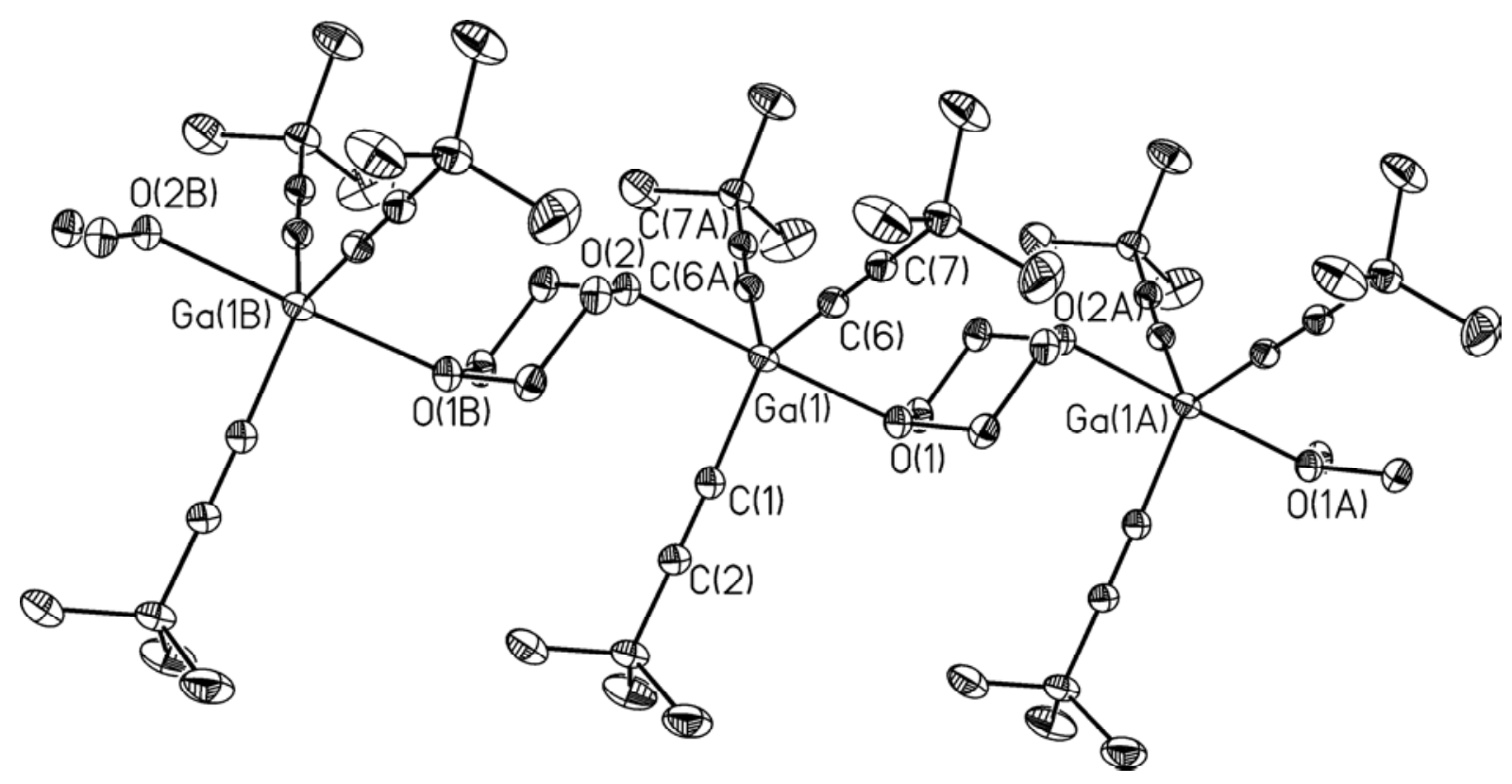

Abb. 14: Struktur von $\mathrm{Ga}(\mathrm{C} \equiv \mathrm{C} t \mathrm{Bu})_{3} \cdot \operatorname{Dioxan}(17)$ im Kristall. Es liegt eine kettenförmige Struktur vor, bei der die Dioxan-Moleküle als verbrückende Einheit fungieren. In jeder Elementarzelle wird ein Galliumatom von zwei halben Dioxan-Molekülen koordiniert.

Ausgewählte Bindungslängen $(\AA)$ und Winkel $\left(^{\circ}\right)$ für 17.

$\begin{array}{llll}\mathrm{Ga}(1)-\mathrm{C}(6) & 1.933(1) & \mathrm{C}(6)-\mathrm{Ga}(1)-\mathrm{O}(1) & 90.81(4) \\ \mathrm{Ga}(1)-\mathrm{C}(1) & 1.934(2) & \mathrm{C}(1)-\mathrm{Ga}(1)-\mathrm{O}(1) & 89.32(6) \\ \mathrm{Ga}(1)-\mathrm{O}(1) & 2.310(1) & \mathrm{C}(6)-\mathrm{Ga}(1)-\mathrm{O}(2) & 88.52(4) \\ \mathrm{Ga}(1)-\mathrm{O}(2) & 2.312(1) & \mathrm{C}(1)-\mathrm{Ga}(1)-\mathrm{O}(2) & 91.99(6) \\ \mathrm{C}(1)-\mathrm{C}(2) & 1.205(3) & \mathrm{O}(1)-\mathrm{Ga}(1)-\mathrm{O}(2) & 178.68(4) \\ \mathrm{C}(7)-\mathrm{C}(6) & 1.209(2) & \mathrm{C}(2)-\mathrm{C}(1)-\mathrm{Ga}(1) & 178.54(17) \\ \mathrm{C}(6) \mathrm{A}-\mathrm{Ga}(1)-\mathrm{C}(6) & 118.81(8) & \mathrm{C}(7)-\mathrm{C}(6)-\mathrm{Ga}(1) & 177.97(12) \\ \mathrm{C}(6)-\mathrm{Ga}(1)-\mathrm{C}(1) & 120.59(4) & & \end{array}$

Im Gegensatz zu 13 liegt in 17 das Galliumatom fünffach koordiniert vor. Dies ist ungewöhnlich, da Gallium in der Regel mit Lewis-Basen die vierfache Koordination anstrebt. ${ }^{[62]}$ Entsprechend der Koordinationszahl fünf bildet $\mathrm{Ga}(\mathrm{C} \equiv \mathrm{C} t \mathrm{Bu})_{3} \cdot$ Dioxan (17) eine trigonale Bipyramide aus, bei der das Gallium trigonal-planar von den Ethinyl-Gruppen umgeben ist. Die Sauerstoffatome der beiden Dioxan-Liganden besetzen die axiale Position. Kein Winkel mit Gallium als zentrales Element (X-Ga-X) weicht mehr als $1.5^{\circ}$ von dem

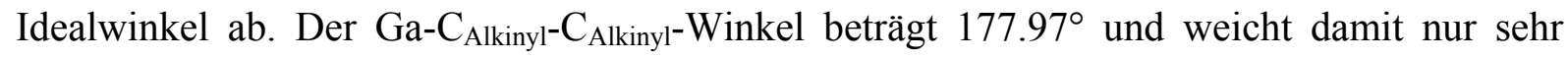




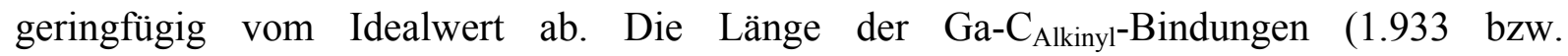
$1.934 \AA$ ) sowie der C $\equiv \mathrm{C}$-Bindungen (1.205 bzw. $1.209 \AA$ ) ist mit denen bekannter GalliumEthinyl-Verbindungen vergleichbar (vgl. Tab. 3). ${ }^{[57,58,59,60]}$

\subsubsection{Darstellung von $\operatorname{In}(\mathrm{C} \equiv \mathrm{C} t \mathrm{Bu})_{3} \cdot \mathrm{NEt}_{3}$ (18)}

$\mathrm{Zu}$ einer Lösung aus $\mathrm{InCl}_{3}$-Lösung in $\mathrm{NEt}_{3}$ wurden bei $-78^{\circ} \mathrm{C}$ drei Äquivalente $\mathrm{Li}-\mathrm{C} \equiv \mathrm{C} t \mathrm{Bu}$ gegeben. Die Reaktionsmischung wurde langsam auf Raumtemperatur erwärmt und 24 Stunden lang gerührt. Anschließend wurde zu der Mischung THF gegeben und über Nacht gerührt. Das Lösungsmittel wurde im Vakuum entfernt und das Produkt mit Hexan extrahiert. Man erhielt 18 als farblosen Feststoff (83\%).

Geeignete Kristalle für die Röntgenstrukturanalyse konnten aus einer Hexanlösung bei $12{ }^{\circ} \mathrm{C}$ gewonnen werden.

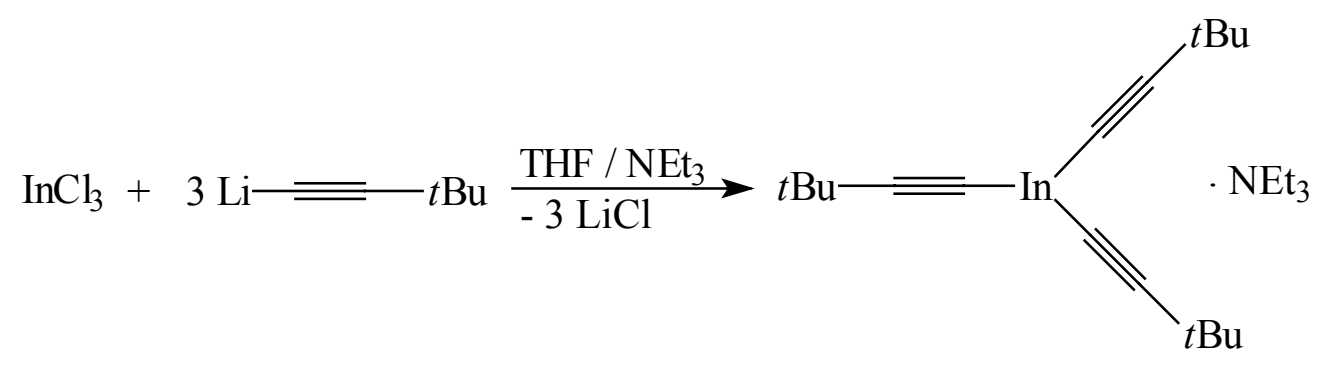

18

Schema 34: Darstellung von 18.

Im ${ }^{1} \mathrm{H}-\mathrm{NMR}-$ Spektrum sind die Resonanzen der Protonen des koordinierenden $\mathrm{NEt}_{3}(\delta 1.08$; 2.66) zu sehen, ebenso das erwartete Singulett der Protonen der $t \mathrm{Bu}-\mathrm{Gruppen}$ bei $\delta 1.19$ mit einer relativen Intensität von 27. Das ${ }^{13}$ C-NMR-Spektrum zeigt das breite Signal (90.3) der an das Indium gebundenen Kohlenstoffatome, das der in $\beta$-Position zum Indium gebundenen Ethinyl-Kohlenstoffatome ( $\delta$ 119.2) sowie die Resonanzen der Kohlenstoffatome der $t \mathrm{Bu}-$ und des koordinierenden $\mathrm{NEt}_{3}$ Liganden. Die Elementaranalyse bestätigt die gefundene Zusammensetzung $\mathrm{C}_{24} \mathrm{H}_{42} \mathrm{InN}$. 


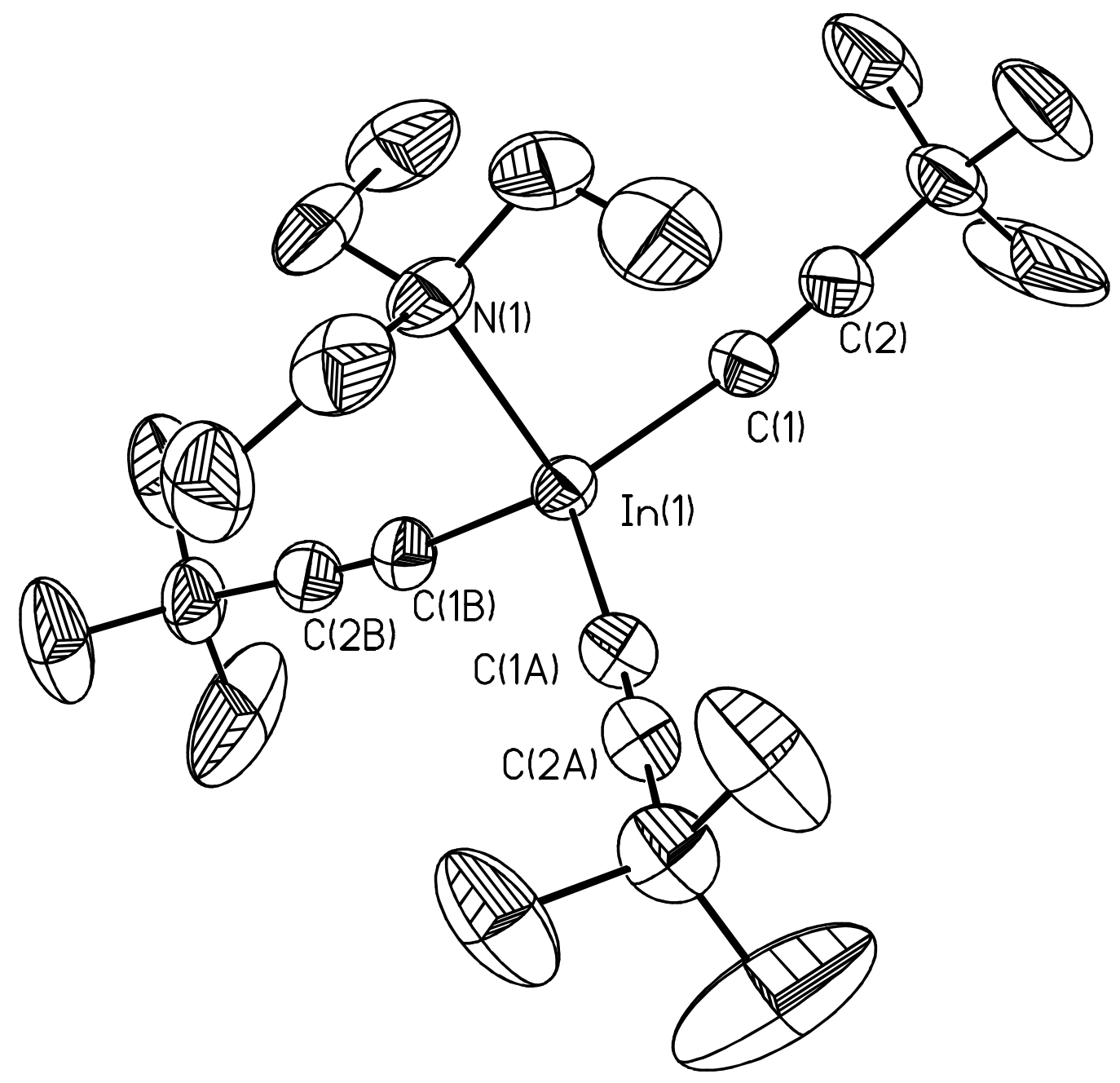

Abb. 15: Struktur von 18 $\mathbf{0 . 5}$ Hexan im Kristall. Das Kristallsolvat (Hexan) ist der Übersichtlichkeit halber nicht abgebildet.

Ausgewählte Bindungslängen $(\AA)$ und Winkel $\left(^{\circ}\right)$ für 18 • 0.5 Hexan.
$\operatorname{In}(1)-C(1) B$
$2.116(3)$
$\mathrm{C}(1) \mathrm{A}-\operatorname{In}(1)-\mathrm{C}(1)$
$116.36(5)$
$\operatorname{In}(1)-\mathrm{N}(1)$
$2.312(4)$
$\mathrm{C}(1) \mathrm{A}-\operatorname{In}(1)-\mathrm{N}(1)$
$101.14(8)$
$\mathrm{C}(1)-\mathrm{C}(2)$
$1.201(4)$
$\mathrm{C}(2)-\mathrm{C}(1)-\operatorname{In}(1)$
$172.3(3)$

In 18 ist das Indiumatom vierfach koordiniert. Neben den drei Ethinyl-Liganden ist die Lewis-Base $\mathrm{NEt}_{3}$ an das Indiumatom koordiniert. Wie u.a. in Verbindung 13 zu beobachten war, verkleinert sich der Winkel zwischen der Lewis-Base und den kovalent gebundenen Liganden (N-In-C-Winkel 101.14). Dies hängt mit der Annäherung des Elektronenzustandes des Indiums an einen $\mathrm{sp}^{2}$-Zustand zusammen. Das System weicht so der sterischen Abstoßung der dichter an das Indium gebundenen Bindungspartner aus (vgl. auch Kap. 2.5.3.). ${ }^{[71,72]}$ Die Lewis-Base tritt mit einem Orbital mit höherem p-Charakter in Wechselwirkung und nimmt 
daher einen Winkel $<109^{\circ}$ ein. Entsprechend sind die C-In-C-Bindungswinkel mit $116.36^{\circ}$ größer als der Tetraeder-Winkel.

Der C-In-Abstand von $2.116 \AA$ sowie der In-C $\equiv \mathrm{C}$-Bindungswinkel $\left(172.3^{\circ}\right)$ ist mit den anderen strukturell charakterisierten Indium-Ethinyl-Verbindungen (vgl. Tab. 4) vergleichbar. Der In-N-Abstand (2.312 $\AA$ ) ist ebenfalls mit den in anderen Verbindungen auftretenden Abständen (z.B. $2.410 \AA\left(\mathrm{Br}_{2} \operatorname{InN}\left(\mathrm{CH}_{2} \mathrm{CH}_{2} \mathrm{NEt}_{2}\right)_{2}\right)^{[76]}$ oder $\left.2.380 \AA\left(\mathrm{Me}_{2} \operatorname{In}\left(\mathrm{CH}_{2}\right)_{3} \mathrm{NMe}_{2}\right)\right)^{[77]}$ vergleichbar.

\subsection{Darstellung von Metall-Tetraethinyl-Verbindungen des Typs $\left[\mathbf{L i} \cdot \mathbf{n} \operatorname{THF}\left(\mathbf{M}(\mathrm{C} \equiv \mathbf{R})_{4}\right)\right](\mathrm{M}=\mathrm{Al})$}

\subsubsection{Darstellung von $\left[\mathrm{Li} \cdot \mathrm{THF}\left(\mathrm{Al}(\mathrm{C} \equiv \mathrm{CtBu})_{4}\right)\right](19)$}

Die Umsetzung von vier Äquivalenten $t$ Butylethin mit $\mathrm{LiAlH}_{4}$ in THF bei Raumtemperatur lieferte unter Wasserstoff-Freisetzung das Produkt 19.

Das ${ }^{1}$ H-NMR- Spektrum zeigt ein Singulett bei $\delta 1.16$, während die Signale der THFProtonen als Multiplett bei $\delta 1.77$ und $3.62 \mathrm{zu}$ sehen sind. Im ${ }^{13} \mathrm{C}-\mathrm{NMR}$ - Spektrum ist eine breite Resonanz bei $\delta 96.3 \mathrm{zu}$ sehen, welche den Alkinyl-Kohlenstoffatomen am Aluminium zuzuordnen ist. Das ${ }^{7}$ Li-NMR- Spektrum zeigt eine scharfe Resonanz bei $\delta 0.04$.

\subsection{Umsetzung der Verbindungen $4,15,19$ und [ $\left.\mathrm{Li} \cdot 2 \mathrm{THF}\left(\mathrm{Al}(\mathrm{C} \equiv \mathrm{CPh})_{4}\right)\right]$ mit $\mathrm{Cp}_{2} \mathrm{ZrMe}_{2}$}

\subsubsection{Umsetzung von ionogenen Aluminium-Ethinyl-Verbindungen mit $\mathrm{Cp}_{2} \mathrm{ZrMe}_{2}$}

Die Reaktion von 4 mit $\mathrm{Cp}_{2} \mathrm{ZrMe}_{2}$ in DME bei Raumtemperatur führt zu keiner Reaktion.

Eine Variation bei den Aluminium-Alkinyl-Verbindungen bringt das gleiche Ergebnis:

Die Umsetzung von 19 mit $\mathrm{Cp}_{2} \mathrm{ZrMe}_{2}$ in $\mathrm{DME}$ bei Raumtemperatur führte zu keiner Reaktion.

Ebenso wenig reagiert $\mathrm{LiAl}(\mathrm{C} \equiv \mathrm{CPh})_{4} \cdot 2 \mathrm{THF}$ mit $\mathrm{Cp}_{2} \mathrm{ZrMe}_{2}$ in $\mathrm{DME}$ bei $80{ }^{\circ} \mathrm{C}$. 


\subsubsection{Umsetzung von neutralen Aluminium-Ethinyl-Verbindungen mit $\mathrm{Cp}_{2} \mathrm{ZrMe}_{2} \mathrm{zu}$} $\mathrm{Cp}_{2} \mathrm{Zr}(\mathrm{C} \equiv \mathrm{C} t \mathrm{Bu})_{2}$

$\mathrm{Al}(\mathrm{C} \equiv \mathrm{C} t \mathrm{Bu})_{3} \cdot$ Dioxan (15) wurde mit einem Äquivalent $\mathrm{Cp}_{2} \mathrm{ZrMe}_{2}$ in THF bei Raumtemperatur umgesetzt. Nachdem das Lösungsmittel im Vakuum entfernt worden ist, wurde der Rückstand mit Toluol versetzt und der resultierende weiße Niederschlag abfiltriert. Das Lösungsmittel des Filtrats wurde anschließend im Vakuum entfernt und der Rückstand mit THF versetzt. Es bildeten sich nach fünf Tagen Kristalle, die als $\mathrm{Cp}_{2} \mathrm{Zr}(\mathrm{C} \equiv \mathrm{C} t \mathrm{Bu})_{2}$ (20) identifiziert werden konnten. Das übrige Produktgemisch konnte nicht weiter aufgetrennt werden.

$$
\mathrm{Cp}_{2} \mathrm{ZrMe}_{2}+\mathrm{Al}(\mathrm{C} \equiv \mathrm{C} t \mathrm{Bu})_{3} \cdot \text { Dioxan } \rightarrow \mathrm{Cp}_{2} \mathrm{Zr}(\mathrm{C} \equiv \mathrm{C} t \mathrm{Bu})_{2}+\text { Produktgemisch }
$$

Schema 35: Darstellung von $\mathrm{Cp}_{2} \mathrm{Zr}(\mathrm{C} \equiv \mathrm{C} t \mathrm{Bu})_{2}$ (20)

Das ${ }^{1} \mathrm{H}-\mathrm{NMR}-$ Spektrum zeigt zwei Singuletts bei $\delta 6.10$ und 1.25 im Intensitätsverhältnis von 10:18, welche den zwei Cp-Liganden und den zwei $t$ Bu-Liganden zugeordnet werden können. Aufgrund der nur geringen Substanzmenge konnten keine weiteren spektroskopischen Untersuchungen durchgeführt werden.

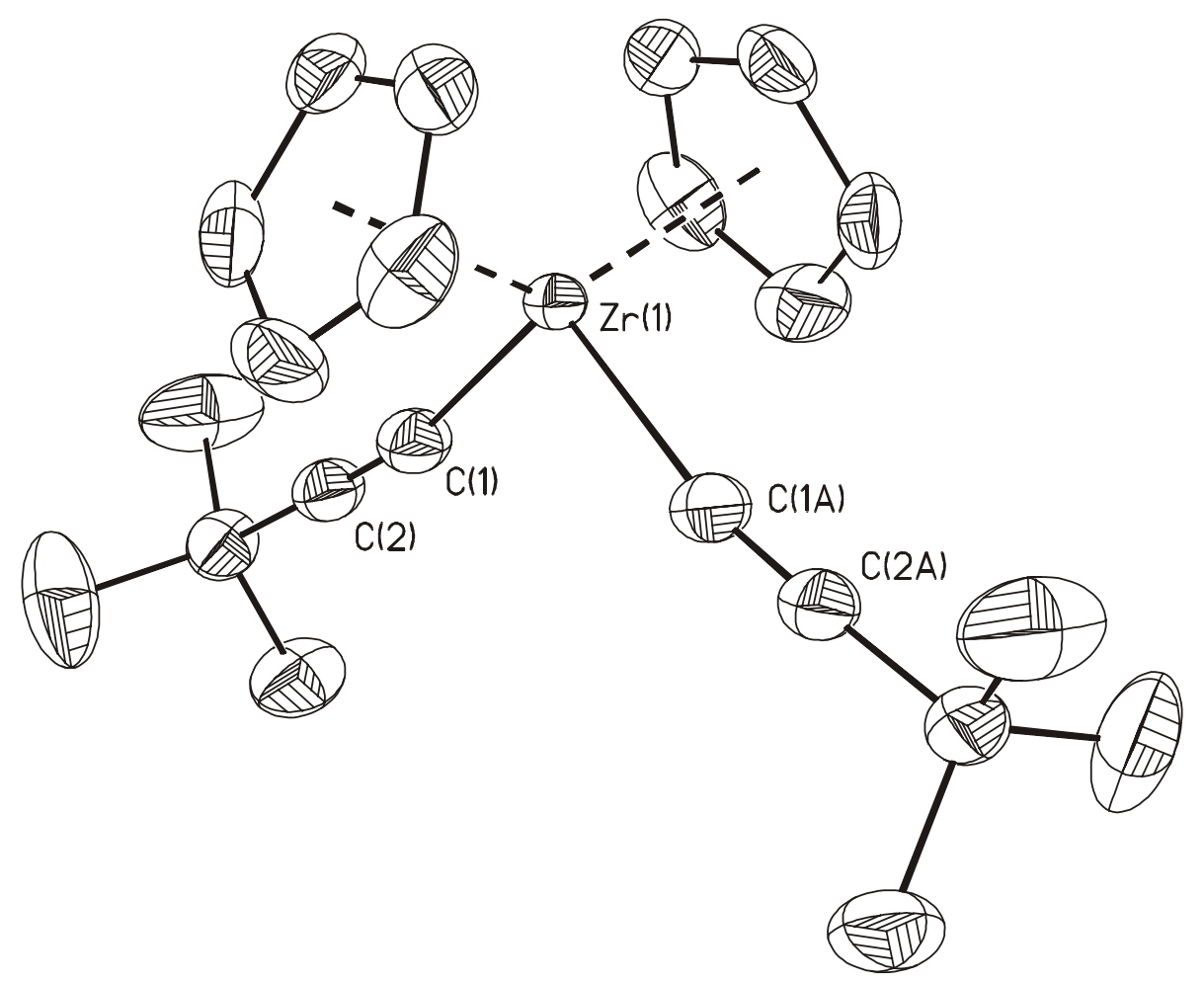

Abb. 16 : Struktur von 20 im Kristall. 
Ausgewählte Bindungslängen $(\AA)$ und Winkel $\left(^{\circ}\right)$ für 20.
$\operatorname{Zr}(1)-\mathrm{C}(1)$
$\mathrm{C}(1)-\mathrm{Zr}(1)-\mathrm{C}(1) \mathrm{A}$
$107.95(13)$
$\mathrm{C}(1)-\mathrm{C}(2)$
$1.208(4)$
$\mathrm{C}(2)-\mathrm{C}(1)-\mathrm{Zr}(1)$
$170.0(2)$

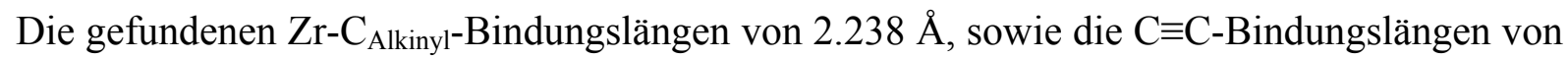
$1.208 \AA \AA$ liegen im Bereich anderer Alkinyl-substituierter Zirconocen-Verbindungen ${ }^{[33,78,79]}$ (z.B. in $\mathrm{Cp}_{2} \mathrm{Zr}(\mathrm{C} \equiv \mathrm{CMe})_{2}$ : $\mathrm{Zr}-\mathrm{C}_{\text {Alkinyl }} 2.249 \AA ; \mathrm{C} \equiv \mathrm{C}$-Bindungslänge $1.206 \AA^{[79]}$ oder in $\mathrm{Cp}_{2} \mathrm{Zr}(\mathrm{C} \equiv \mathrm{CnBu})_{2}: \quad \mathrm{Zr}-\mathrm{C}_{\text {Alkinyl }} \quad 2.241 \quad \AA ; \quad \mathrm{C} \equiv \mathrm{C}$-Bindungslänge $\left.\quad 1.198 \quad \AA\right) .{ }^{[78]} \quad$ Der $\mathrm{C}(1)-\mathrm{Zr}(1)-\mathrm{C}(1) \mathrm{A}-W i n k e l\left(108.0^{\circ}\right)$ ist etwas größer als bei $\mathrm{Cp}_{2} \mathrm{Zr}(\mathrm{C} \equiv \mathrm{C}-\mathrm{Me})_{2}\left(103.6^{\circ}\right)^{[79]}$ oder bei $\mathrm{Cp}_{2} \mathrm{Zr}(\mathrm{C} \equiv \mathrm{CnBu})_{2}\left(105.4^{\circ}\right),{ }^{[78]}$ was mit der größeren Abstoßung aufgrund des sterisch anspruchsvolleren Restes erklärt werden kann.

\subsection{Darstellung einer tetrameren Aluminium(+I)-Verbindung}

\subsubsection{Darstellung von $\left[\mathrm{RAII}_{2}\right]_{2}(21) \quad\left(\mathrm{R}=2,6-i \mathrm{Pr}_{2} \mathrm{C}_{6} \mathrm{H}_{3} \mathrm{~N}\left(\mathrm{SiMe}_{3}\right)\right)$}

Im Gegensatz zu den Chloriden und Bromiden sind bisher nur wenige Organo-AluminiumIodide untersucht worden (vgl. Tab. 5, Seite 53). ${ }^{[20,80,81,82]}$ Bei den bekannten Verbindungen handelt es sich meist um basenstabilisierte, monomere Aluminium-Diiodide. Im Folgenden wird die Darstellung und Struktur des ersten basenfreien, dimeren Organo-AluminiumDiiodids beschrieben, bei der zwei Iodatome als verbrückende Einheit fungieren:

$\mathrm{Zu}$ der basenfreien Aluminium-Dimethyl-Verbindung $\left[\mathrm{RAlMe}_{2}\right]_{2}\left(\mathrm{R}=2,6-i \mathrm{Pr}_{2} \mathrm{C}_{6} \mathrm{H}_{3} \mathrm{~N}\left(\mathrm{SiMe}_{3}\right)\right)$ wurden in einem nicht-koordinierenden Lösungsmittel (Toluol) bei $-78{ }^{\circ} \mathrm{C}$ vier Äquivalente Iod zugegeben und über Nacht bei Raumtemperatur unter Lichtausschluss gerührt. Nach Entfernen des Lösungsmittels im Vakuum und anschließendem Waschen mit Pentan erhielt man die gewünschte Verbindung $\left[\mathrm{RAlI}_{2}\right]_{2} \quad \mathbf{( 2 0 )}$. Durch langsames Entfernen des Lösungsmittels Toluol konnten für eine Röntgenstrukturanalyse geeignete Einkristalle erhalten werden.

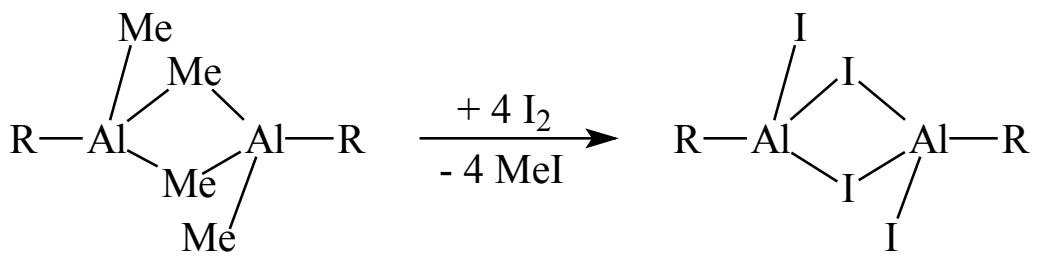

Schema 36: Darstellung der ersten basenfreien, iodidverbrückten Aluminium-Diiodid-Verbindung 21 
Das ${ }^{1} \mathrm{H}-\mathrm{NMR}-$ Spektrum zeigt ein Multiplett bei $\delta$ 7.0-7.1, was den Aryl-Protonen zuzuordnen ist. Die CH-Protonen ergeben ein Septett ( $\delta$ 3.55), während die Methylprotonen als zwei Dubletts bei $\delta 1.35$ bzw. 1.22 zu sehen sind. Die $\mathrm{SiMe}_{3}$-Protonen sind im Spektrum als Singulett bei $\delta 0.34 \mathrm{zu}$ sehen. Das Massenspektrum zeigt den Molpeak des Monomers (529).

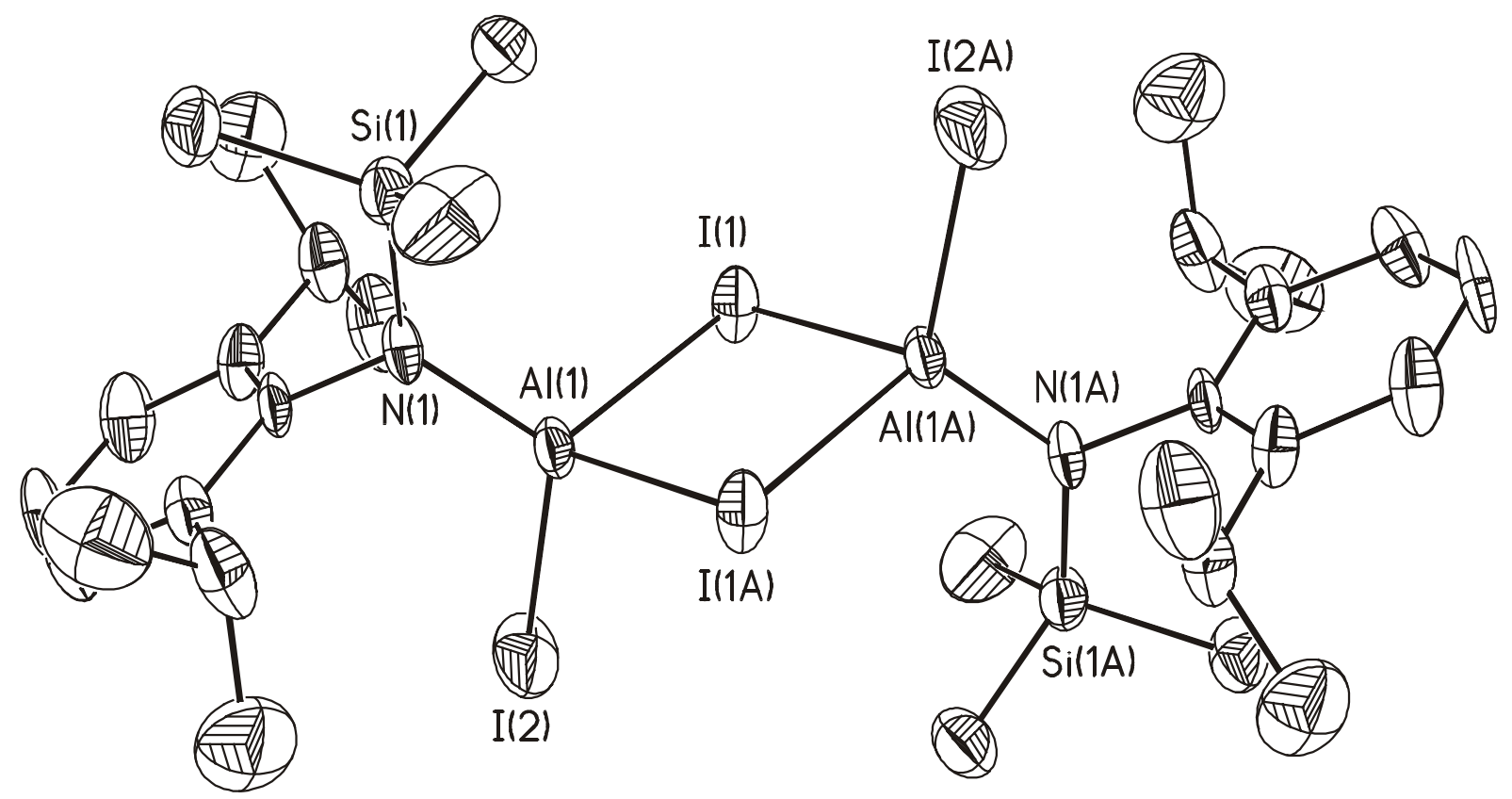

Abb. 17: Struktur von 21 im Kristall.

Ausgewählte Bindungslängen $(\AA)$ und Winkel $\left(^{\circ}\right)$ für 21.

$\begin{array}{lclc}\mathrm{Al}(1)-\mathrm{N}(1) & 1.784(6) & \mathrm{N}(1)-\mathrm{Al}(1)-\mathrm{I}(1) \mathrm{A} & 113.34(19) \\ \mathrm{Al}(1)-\mathrm{I}(2) & 2.464(2) & \mathrm{I}(2)-\mathrm{Al}(1)-\mathrm{I}(1) \mathrm{A} & 106.81(8) \\ \mathrm{Al}(1)-\mathrm{I}(1) \mathrm{A} & 2.645(2) & \mathrm{N}(1)-\mathrm{Al}(1)-\mathrm{I}(1) & 114.7(2) \\ \mathrm{Al}(1)-\mathrm{I}(1) & 2.687(2) & \mathrm{I}(2)-\mathrm{Al}(1)-\mathrm{I}(1) & 103.92(8) \\ \mathrm{I}(1)-\mathrm{Al}(1) \mathrm{A} & 2.645(2) & \mathrm{I}(1) \mathrm{A}-\mathrm{Al}(1)-\mathrm{I}(1) & 93.83(7) \\ \mathrm{N}(1)-\mathrm{Al}(1)-\mathrm{I}(2) & 120.59(19) & & \end{array}$

Die Verbindung $\left[\mathrm{RAlI}_{2}\right]_{2}$ (21) liegt in kristalliner Form als Dimer vor. Dies war aufgrund der zuvor dargestellten isostrukturellen Verbindung $\left[\mathrm{RAlCl}_{2}\right]_{2}$ (3) zu erwarten. Aus der Röntgenstrukturanalyse geht hervor, dass die tetraedrische Koordination am Aluminium-Atom verzerrt ist. So beträgt der I(1)A-Al(1)-I(1)-Winkel nur 93.83, während der N(1)-Al(1)-I(2)Winkel $120.59^{\circ}$ beträgt. Die Al-I-Bindungslängen der verbrückt gebundenen Iodatome sind deutlich länger (2.645-2.687 $\AA$ ) als die der bekannten Bindungslängen von RAlI $2^{-}$ Verbindungen, während die Al-I-Bindungslängen $\mathrm{zu}$ den terminal gebundenen Iodatomen 
(2.464 ̊) deutlich kürzer sind (vergleiche Tab. 5). Dies steht im Einklang mit anderen Untersuchungen aus denen hervorgeht, dass die Bindungslängen in verbrückt gebundenen Atomen, von wenigen speziellen Ausnahmen abgesehen, länger sind. ${ }^{[41]}$ Wie bei den solvatfreien isostrukturellen Aluminium-Dichloriden ${ }^{[40,42,41]}$ (vgl. Tab. 2, bzw. vgl. Verbindung 3) sollte $\left[\mathrm{RAll}_{2}\right]_{2}$ (21) ebenfalls deutlich reaktiver sein als die basenkoordinierten Verbindungen, um eine Vielzahl an Folgereaktionen durchführen zu können. Der größte Vorteil jedoch, neben der höheren Aktivität, ist der Ausschluss an Oxidationsprodukten, die z.B. bei THF-Addukten auftreten können. ${ }^{[19,20]}$

Tabelle 5: Übersicht über strukturell charakterisierte Aluminiumdiiodid-Verbindungen:

\begin{tabular}{|l|l|}
\hline & Al-I-Bindungslängen $(\AA)$ \\
\hline$\left(\mathrm{SiMe}_{3}\right)_{3} \mathrm{CAlI}_{2} \cdot \mathrm{THF}^{[82]}$ & 2.564 \\
\hline $\mathrm{Cp}^{*} \mathrm{AlI}_{2} \cdot \mathrm{OEt}_{2}{ }^{[83]}$ & $2.535-2.548$ \\
\hline $\operatorname{tmpAlI}_{2} \cdot \mathrm{py}^{[81]}$ & $2.521-2.535$ \\
\hline$\left[\mathrm{Cp}^{*} \mathrm{IAlAlI}_{3}\right]\left[\left(\mathrm{Cp}^{*} \mathrm{Al}\right)_{2} \mathrm{AlI}_{2}\right]^{[84]}$ & $2.538-2.568^{\mathrm{x}}$ \\
\hline $\mathrm{Et}_{2} \mathrm{O} \cdot \mathrm{I}_{2} \mathrm{AlAlI}_{2} \cdot \mathrm{OEt}_{2}{ }^{[85]}$ & $2.542-2.543$ \\
\hline$\left(\mathrm{C}_{12} \mathrm{H}_{19} \mathrm{~N}_{2}\right) \mathrm{AlI}_{2}{ }^{[20]}$ & $2.544-2.559$ \\
\hline$\left[\left(\mu-\mathrm{NMe}_{2}\right) \mathrm{AlI}_{2}\right]_{2}{ }^{[80]}$ & $2.478-2.506$ \\
\hline$\left[\left(\mu-\mathrm{OSiH}(\mathrm{Me})_{2}\right) \mathrm{AlI}_{2}\right]_{2}^{[86]}$ & $2.469-2.477$ \\
\hline
\end{tabular}

${ }^{\mathrm{x}}$ Bindungslängen der $\mathrm{AlI}_{2}$-Gruppe im Anion

\subsubsection{Darstellung der tetrameren Aluminium(+I)-Verbindung $[\mathrm{RAI}]_{4} \cdot$ Hexan $(22 \cdot \mathrm{Hexan})\left(\right.$ mit $\left.\mathrm{R}=2,6-i \mathrm{Pr}_{2} \mathrm{C}_{6} \mathrm{H}_{3} \mathrm{~N}\left(\mathrm{SiMe}_{3}\right)\right)$}

Ein Beispiel für eine wichtige Reaktion eines bekannten Aluminium-Diiodids ist die Reduktion mit $\mathrm{Na} / \mathrm{K}$ - Legierung, bei der neben dem Hauptprodukt einer Al(+I)-Verbindung als Nebenprodukt das THF-Oxidationsprodukt entsteht. ${ }^{[19]}$ Dies senkt die maximal mögliche Ausbeute an Hauptprodukt stark ab. Durch die Basenkoordination ist außerdem die Reaktivität der bekannten Diiodide herabgesetzt und erfordert für die Reduktion mit der $\mathrm{Na} / \mathrm{K}$-Legierung den Einsatz drastischerer Reaktionsbedingungen.

Da den niedervalenten Aluminium-Verbindungen eine sehr wichtige Rolle bei der Darstellung neuer Koordinations-Verbindungen mit d-Metallen zukommt, ${ }^{[87]}$ die wiederum eine wichtige Rolle bei der Metallorganischen Chemischen Dampfabscheidung (MOCVD) spielen, wurde der 
Versuch unternommen, eine starke, sterisch anspruchsvolle Stickstoffbasen-stabilisierte tetramere Aluminium(+I)-Verbindung darzustellen.

$\mathrm{Zu}$ diesem Zweck wurde (21) dargestellt, deren Reduktion mit $\mathrm{Na} / \mathrm{K}$-Legierung bei schonenden Bedingungen (Raumtemperatur in Hexan) nach Umkristallisation in Diisopropylether in mäßiger Ausbeute $(32 \%)$ zu der tetrameren Aluminium $(+\mathrm{I})$-Verbindung $(\mathrm{RAl})_{4}(\mathbf{2 2} \cdot$ Dippe) (mit $\mathrm{R}=2,6-i \mathrm{Pr}_{2} \mathrm{C}_{6} \mathrm{H}_{3} \mathrm{~N}\left(\mathrm{SiMe}_{3}\right)$ ) führte.

Der vermessene Einkristall wurde schon vor der Aufarbeitung mit Diisopropylether in einer Hexanlösung erhalten und konnte mittels einer Röntgenstrukturanalyse als Hexan-Addukt (22 - Hexan) charakterisiert werden. Aus dem ${ }^{1} \mathrm{H}-\mathrm{NMR}-\mathrm{Spektrum}$ geht hervor, dass nach der Umkristallisation in Diisopropylether dieses das Hexan aus der Koordinationssphäre von 22 verdrängt hat und die Verbindung als Diisopropylether-Addukt (22 • Dippe) vorliegt.

Da keine Variationen bezüglich des Lösungsmittels, der Reaktionsbedingungen oder des Reduktionsmittels durchgeführt wurden, sollte eine deutliche Ausbeute-Steigerung möglich sein.

Nach der Entdeckung der ersten monomeren Stickstoffbasen-stabilisierten Al( $+\mathrm{I})$-Verbindung in unserem Arbeitskreis ${ }^{[8]}$ erhielt man nun die erste, strukturell charakterisierte, nur von Stickstoffbasen stabilisierte tetramere Aluminium(+I)-Verbindung $(\mathbf{2 2} \cdot \mathbf{H e x a n})$.<smiles>[R]I(I)[Al]([R])(I)I</smiles>

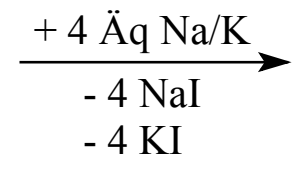

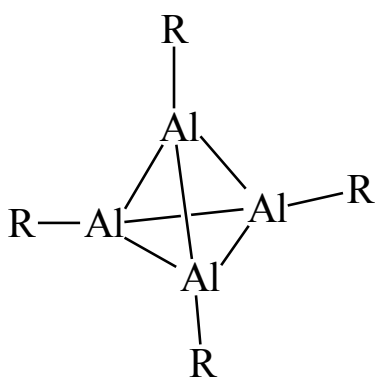

$$
\mathrm{R}=2,6-i \mathrm{Pr}_{2} \mathrm{C}_{6} \mathrm{H}_{3} \mathrm{~N}\left(\mathrm{SiMe}_{3}\right)
$$

Schema 37: Darstellung der tetrameren Aluminium(+I)-Verbindung $(22 \cdot$ Hexan $)$

Das ${ }^{1}$ H-NMR-Spektrum bei Standardtemperatur $(300 \mathrm{~K})$ zeigt sehr breite Signale, was mit der gehinderten Rotation aufgrund der sterischen Hinderung und damit der chemischen Nichtäquivalenz zu erklären ist. Ein Hochtemperatur- ${ }^{1}$ H-NMR-Spektrum bei $343 \mathrm{~K}$ mildert diesen Effekt, so dass die Kopplungen der CH-Protonen mit den Methyl-Protonen der $i$ Pr-Gruppe bestimmbar waren $(J=6.9 \mathrm{~Hz})$. 
Im Standard- ${ }^{1} \mathrm{H}-\mathrm{NMR}-$ Spektrum erzeugen die 12 Aryl-Protonen bei $\delta 7.05$ ein Signal mit einem $\mathrm{AB}_{2}$-Kopplungsmuster. Die vier Protonen der $\mathrm{CH}-\mathrm{Gruppen}$ ergeben bei $\delta 3.68$ ein breites Signal, während acht Methylgruppen-Protonen bei $\delta 1.27$, vier Methylgruppen-Protonen bei $\delta 1.14$ und vier Methylgruppen-Protonen bei $\delta 1.06$ in Resonanz sind. Dies spricht für eine chemisch unterschiedliche Umgebung der Methylgruppen-Protonen einer $i$ Pr-Gruppe (s. o.). Die Protonen der vier $\mathrm{SiMe}_{3}$-Gruppen erzeugen ein breites Singulett bei $\delta 0.20$. Außerdem sind die bekannten Signale der Protonen des Diisopropylethers zu sehen $(\delta=1.06,3.45)$. Das ${ }^{13} \mathrm{C}$-NMR-Spektrum zeigt die Resonanzen des 2,6-i $\mathrm{Pr}_{2} \mathrm{C}_{6} \mathrm{H}_{3} \mathrm{~N}\left(\mathrm{SiMe}_{3}\right)$-Liganden sowie des Diisopropylethers bei $\delta=146.2,138.8,124.7,124.1$ (aromatische Kohlenstoffatome), 28.1, 27.5 (CH-Gruppe), 23.8, 23.0 ( $\mathrm{CH}_{3}$-Gruppe), 3.9 ( $\mathrm{Si}\left(\mathrm{CH}_{3}\right)_{3}$-Gruppe).

Das Massenspektrum zeigt den Molpeak (1100) sowie die Fragmente (RAl) 3 (825), $(\mathrm{RAl})_{2}$ (550) und RAl (275). Die Elementaranalyse von 22 - Dippe bestätigt die gefundene Zusammensetzung $\mathrm{C}_{66} \mathrm{H}_{118} \mathrm{Al}_{4} \mathrm{~N}_{4} \mathrm{OSi}_{4}$.

Tabelle 6: Übersicht über alle strukturell charakterisierten, tetrameren Aluminium( $+\mathrm{I})$ Verbindungen:

\begin{tabular}{|l|c|l|l|}
\hline & $\begin{array}{l}\text { Anzahl der } \\
\text { Al-Atome }\end{array}$ & $\begin{array}{l}\text { Al-Al-Bindungs- } \\
\text { länge }(\mathrm{n})(\AA)\end{array}$ & $\begin{array}{l}\text { Al-N-Bindungslänge(n) } \\
(\text { falls vorhanden })\end{array}$ \\
\hline$\left[\mathrm{AlCp}{ }^{*}\right]_{4}^{[89]}$ & 4 & 2.769 & - \\
\hline$\left[\mathrm{AlC}\left(\mathrm{SiMe}_{3}\right)_{3}\right]_{4} \cdot 0.5 \mathrm{Hexan}^{[90]}$ & 4 & 2.739 & - \\
\hline$\left[(\mathrm{AlCp})_{3} \mathrm{AlN}\left(\mathrm{SiMe}_{3}\right)_{2}\right]^{[9]}$ & 4 & $2.632-2.762$ & 1.847 \\
\hline$\left[\mathrm{AlSi}(\mathrm{tBu})_{3}\right]_{4} \cdot \mathrm{Heptan}^{[92]}$ & 4 & $2.6004-2.612$ & - \\
\hline$\left[\mathrm{AlSi}(\mathrm{SiMe})_{3}\right]_{4} \cdot 0.5 \mathrm{Hexan}^{[93]}$ & 4 & $2.592-2.612$ & - \\
\hline
\end{tabular}




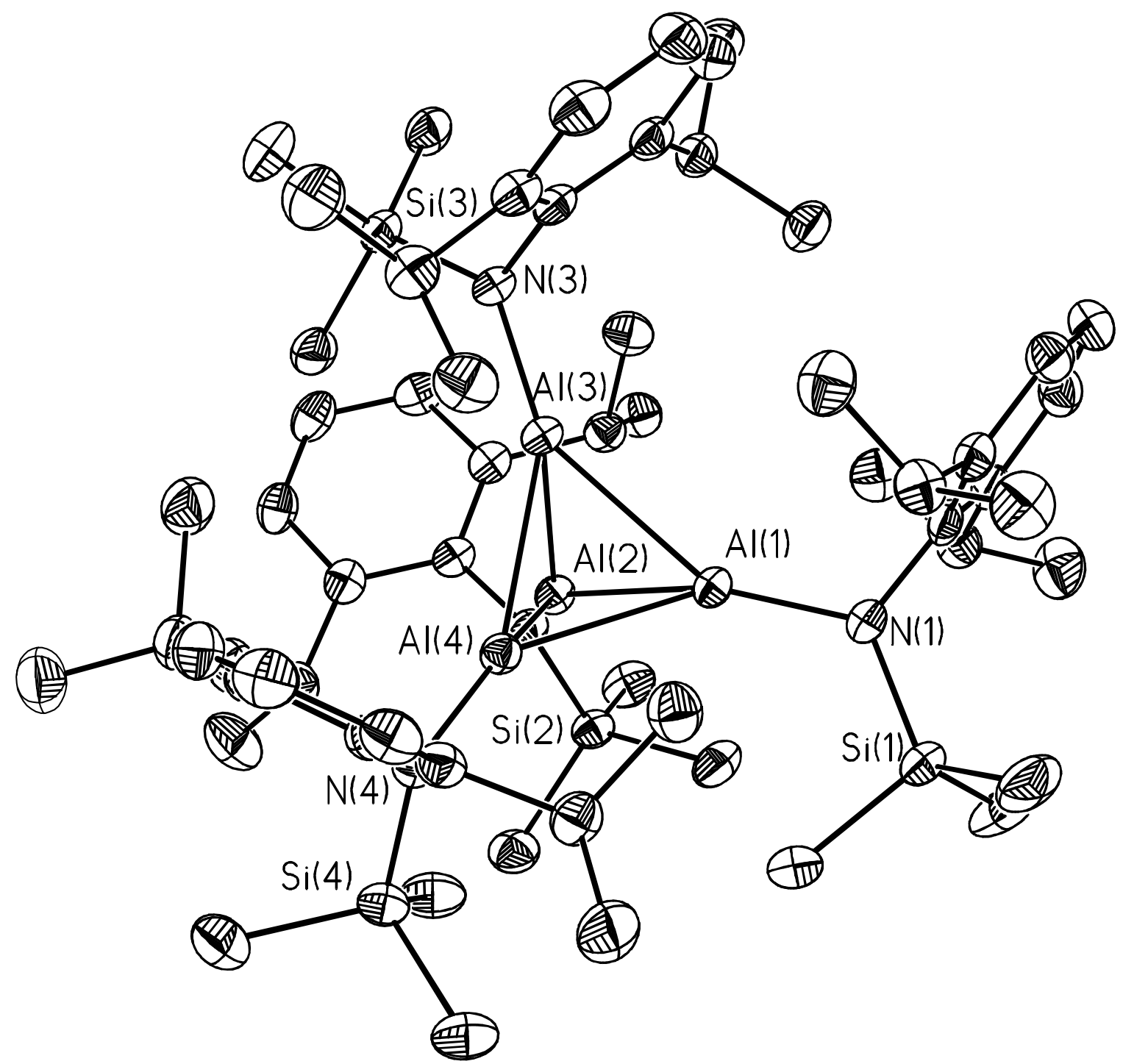

Abb. 18: Struktur von 22 im Kristall. Das Kristallsolvat (Hexan) ist der Übersichtlichkeit halber nicht abgebildet.

Ausgewählte Bindungslängen $(\AA)$ und Winkel $\left(^{\circ}\right)$ für $22 \cdot$ Hexan.

$\begin{array}{llll}\mathrm{Al}(1)-\mathrm{N}(1) & 1.821(2) & \mathrm{Al}(4)-\mathrm{N}(4) & 1.818(2) \\ \mathrm{Al}(1)-\mathrm{Al}(2) & 2.607(1) & \mathrm{N}(1)-\mathrm{Al}(1)-\mathrm{Al}(2) & 142.19(8) \\ \mathrm{Al}(1)-\mathrm{Al}(4) & 2.617(1) & \mathrm{N}(1)-\mathrm{Al}(1)-\mathrm{Al}(4) & 142.24(8) \\ \mathrm{Al}(1)-\mathrm{Al}(3) & 2.635(1) & \mathrm{Al}(2)-\mathrm{Al}(1)-\mathrm{Al}(4) & 60.34(3) \\ \mathrm{Al}(2)-\mathrm{N}(2) & 1.808(2) & \mathrm{N}(1)-\mathrm{Al}(1)-\mathrm{Al}(3) & 149.54(8) \\ \mathrm{Al}(2)-\mathrm{Al}(3) & 2.601(1) & \mathrm{Al}(2)-\operatorname{Al}(1)-\mathrm{Al}(3) & 59.50(3) \\ \mathrm{Al}(2)-\mathrm{Al}(4) & 2.625(1) & \mathrm{Al}(4)-\operatorname{Al}(1)-\mathrm{Al}(3) & 60.01(3) \\ \mathrm{Al}(3)-\mathrm{N}(3) & 1.814(2) & \mathrm{N}(2)-\mathrm{Al}(2)-\mathrm{Al}(3) & 145.61(7) \\ \mathrm{Al}(3)-\mathrm{Al}(4) & 2.627(1) & \mathrm{N}(2)-\mathrm{Al}(2)-\mathrm{Al}(1) & 145.14(7)\end{array}$




$\begin{array}{llll}\mathrm{Al}(3)-\mathrm{Al}(2)-\mathrm{Al}(1) & 60.79(3) & \mathrm{Al}(2)-\mathrm{Al}(3)-\mathrm{Al}(1) & 59.71(3) \\ \mathrm{N}(2)-\mathrm{Al}(2)-\mathrm{Al}(4) & 142.64(8) & \mathrm{Al}(4)-\mathrm{Al}(3)-\mathrm{Al}(1) & 59.66(3) \\ \mathrm{Al}(3)-\mathrm{Al}(2)-\mathrm{Al}(4) & 60.34(3) & \mathrm{N}(4)-\mathrm{Al}(4)-\mathrm{Al}(1) & 142.81(7) \\ \mathrm{Al}(1)-\mathrm{Al}(2)-\mathrm{Al}(4) & 60.03(3) & \mathrm{N}(4)-\mathrm{Al}(4)-\mathrm{Al}(2) & 141.99(8) \\ \mathrm{N}(3)-\mathrm{Al}(3)-\mathrm{Al}(2) & 141.79(7) & \mathrm{Al}(1)-\mathrm{Al}(4)-\mathrm{Al}(2) & 59.63(3) \\ \mathrm{N}(3)-\mathrm{Al}(3)-\mathrm{Al}(4) & 144.06(8) & \mathrm{N}(4)-\mathrm{Al}(4)-\mathrm{Al}(3) & 149.40(8) \\ \mathrm{Al}(2)-\mathrm{Al}(3)-\mathrm{Al}(4) & 60.29(3) & \mathrm{Al}(1)-\mathrm{Al}(4)-\mathrm{Al}(3) & 60.33(3) \\ \mathrm{N}(3)-\mathrm{Al}(3)-\mathrm{Al}(1) & 148.36(7) & \mathrm{Al}(2)-\operatorname{Al}(4)-\mathrm{Al}(3) & 59.37(3)\end{array}$

Der Röntgenkristallstrukturanalyse zufolge liegt ein nahezu perfektes tetraedrisches $\mathrm{Al}_{4}$-Gerüst vor. Kein Al-Al-Al-Winkel weicht um mehr als $1^{\circ}$ vom Idealwinkel $\left(60^{\circ}\right) \mathrm{ab}$. Der $\mathrm{Al}_{4}$-Cluster weist relativ kurze Al-Al-Bindungen mit im Mittel $2.619 \AA$ auf (vgl. Tab. 6). Die Al-N-Bindung mit im Mittel $1.815 \AA$ ist mit anderen bekannten Al-N-Bindungslängen vergleichbar (vgl. Verbindungen 3, 4, 5, 6, 7 (Al-N-Bindungslängen: 1.774-1.837 Å) bzw. vgl. Tab. 6, 7).

Tabelle 7: Weitere strukturell untersuchte Verbindungen mit niedervalentem Aluminium:

\begin{tabular}{|c|c|c|c|}
\hline & $\begin{array}{l}\text { Anzahl der } \\
\text { Al-Atome }\end{array}$ & $\begin{array}{l}\text { Al-Al-Bindungs- } \\
\text { länge(n) }(\AA)\end{array}$ & $\begin{array}{l}\text { Al-N-Bindungslänge(n) } \\
\text { (falls vorhanden) }\end{array}$ \\
\hline$\left[\left\{\mathrm{HC}(\mathrm{CMeNAr})_{2}\right\} \mathrm{Al}\right]^{[88]^{*}}$ & 1 & - & 1.957 \\
\hline$\left[\mathrm{Al}_{3} \mathrm{Si}(\mathrm{tBu})_{4}\right] \cdot 0.25 \mathrm{Hexan}^{[94]}$ & 3 & $2.623-2.902$ & - \\
\hline$\left[\mathrm{Li}\left(\mathrm{OEt}_{2}\right)_{3}\right]\left[\mathrm{Al}_{7}\left(\mathrm{~N}\left(\mathrm{SiMe}_{3}\right)_{2}\right)_{6}\right]^{[95]}$ & 7 & $2.540-2.737$ & 1.844 \\
\hline$\left[\mathrm{Li}\left(\mathrm{OEt}_{2}\right)_{3}\right]\left[\mathrm{Al}_{12}\left(\mathrm{~N}\left(\mathrm{SiMe}_{3}\right)_{2}\right)_{8}\right]^{[96]}$ & 12 & $2.542-2.788$ & 1.848 \\
\hline$\left[\mathrm{K}_{2}(\mathrm{Tol})\right]\left[\mathrm{Al}_{12}(i \mathrm{Bu})_{12}\right]^{[97]}$ & 12 & $2.679-2.696$ & - \\
\hline$\left[\mathrm{Al}_{14} \mathrm{Cp}_{6}{ }_{6} \mathrm{ClSi}\right] \cdot 2 \mathrm{C}_{8} \mathrm{H}_{10}{ }^{[98]}$ & 14 & $2.79-2.90$ & - \\
\hline $\begin{array}{l}{\left[\mathrm{Li}\left(\mathrm{OEt}_{2}\right)_{4}\right]} \\
{\left[\mathrm{Al}_{14}\left(\mathrm{~N}\left(\mathrm{SiMe}_{3}\right)_{2}\right)_{6} \mathrm{I}_{6}\left(\mathrm{OEt}_{2}\right)_{2} \mathrm{Li}\right] \cdot \mathrm{Tol}^{[99]}}\end{array}$ & 14 & $2.57-2.91$ & 1.86 \\
\hline $2\left[\mathrm{Al}_{22} \mathrm{Br}_{20}(\mathrm{THF})_{12}\right] \cdot 16 / 3 \mathrm{Tol}^{[100]}$ & 22 & $2.53-2.76$ & - \\
\hline $\begin{array}{l}{\left[\mathrm{Al}_{69}\left\{\mathrm{~N}\left(\mathrm{SiMe}_{3}\right)_{2}\right\}_{18}\right]\left[\mathrm{Li}\left(\mathrm{OEt}_{2}\right)_{4}\right] \cdot 6} \\
\mathrm{Tol}^{[101]}\end{array}$ & 69 & 2.543-2.991 & keine Angaben \\
\hline $\begin{array}{l}2\left[\mathrm{Li}\left(\mathrm{OEt}_{2}\right)_{3}(\mu-\mathrm{I}) \mathrm{Li}\left(\mathrm{OEt}_{2}\right)_{2}\right] \cdot 2 \text { Tol } \\
{\left[\mathrm{Al}_{77}\left\{\mathrm{~N}\left(\mathrm{SiMe}_{3}\right)_{2}\right\}_{20}\right]^{[102]}}\end{array}$ & 77 & 2.564-2.999 & 1.831 \\
\hline
\end{tabular}

$* \mathrm{Ar}=2,6-i \mathrm{Pr}_{2} \mathrm{C}_{6} \mathrm{H}_{3}$ 


\section{Zusammenfassung und Ausblick}

Ziel dieser Arbeit war es Wege zur Darstellung von Vinyliden-Derivaten der 13. Gruppe des Periodensystems zu untersuchen. Zunächst wurden die Reaktionen von bekannten Übergangsmetallocen-Diethinyl-Verbindungen mit reaktiven Aluminium-Verbindungen wie z. B. monomeren Aluminium-Dihydriden untersucht.

Hier konnte festgestellt werden, dass bei Einsatz von Titanocen-Diethinyl-Verbindungen eine Reduktion zu bekannten Titan(III)-Verbindungen erfolgte. Die Reaktion von ZirconocenDiethinyl-Verbindungen mit reaktiven Aluminiumverbindungen lieferte nicht die erwünschten Produkte, sondern es kam zu literaturbekannten Umlagerungsreaktionen.

Eine weitere Möglichkeit, Zugang zu Vinyliden-Derivaten der 13. Gruppe des Periodensystems zu erlangen, wurde in den Umsetzungen von reaktiven Metallocen-AlkylVerbindungen mit einer Trielverbindung mit zwei oder mehr terminal gebundenen Ethinylgruppen gesehen (vgl. Schema 10).

Für die Darstellung mehrfach Ethinyl-substituierter Aluminiumverbindungen wurde ein Aminodichlor-Alan mit 2,6-i $\operatorname{Pr}_{2} \mathrm{C}_{6} \mathrm{H}_{3} \mathrm{~N}\left(\mathrm{SiMe}_{3}\right)$ als Ligand dargestellt (3).

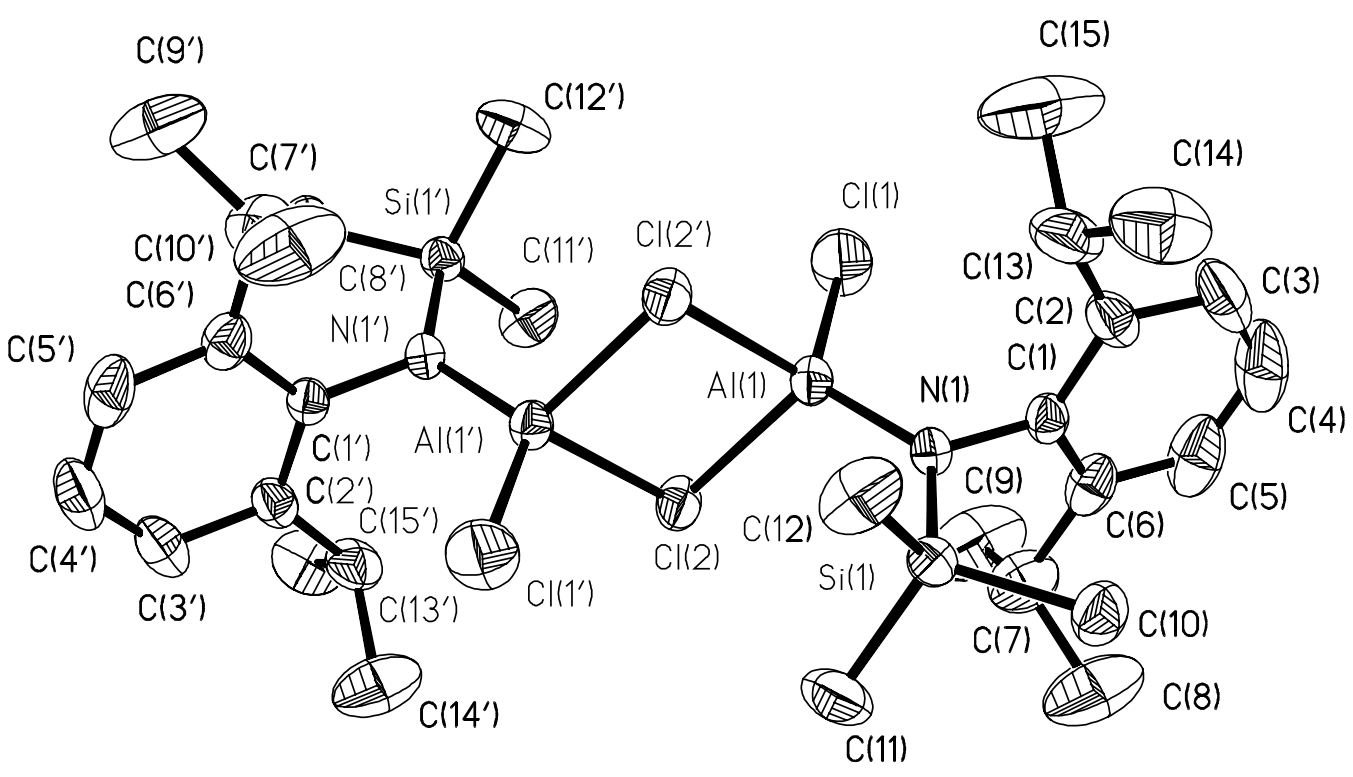


Zunächst konnten durch die Umsetzung von 3 mit drei Äquivalenten eines Alkali-EthinylSalzes die ersten anionischen Aluminiumverbindungen mit drei terminalen Ethinylgruppen dargestellt werden $(\mathbf{4}, \mathbf{5}, \mathbf{6})$. Bemerkenswerterweise koordinieren die Kationen $\left(\mathrm{K}^{+}(\mathbf{4})\right.$, $\left.\mathrm{Na}^{+}(\mathbf{5}), \mathrm{Li}^{+}(\mathbf{6})\right)$ an die elektronenreichen Ethinylgruppen.

Die Verbindungen $\mathbf{4}$ und $\mathbf{5}$ sind isostrukturell aufgebaut und bilden durch zwei verbrückende Kationen ein Dimer. Damit sind die Kationen von vier Ethinylgruppen und einem THFMolekül koordiniert.

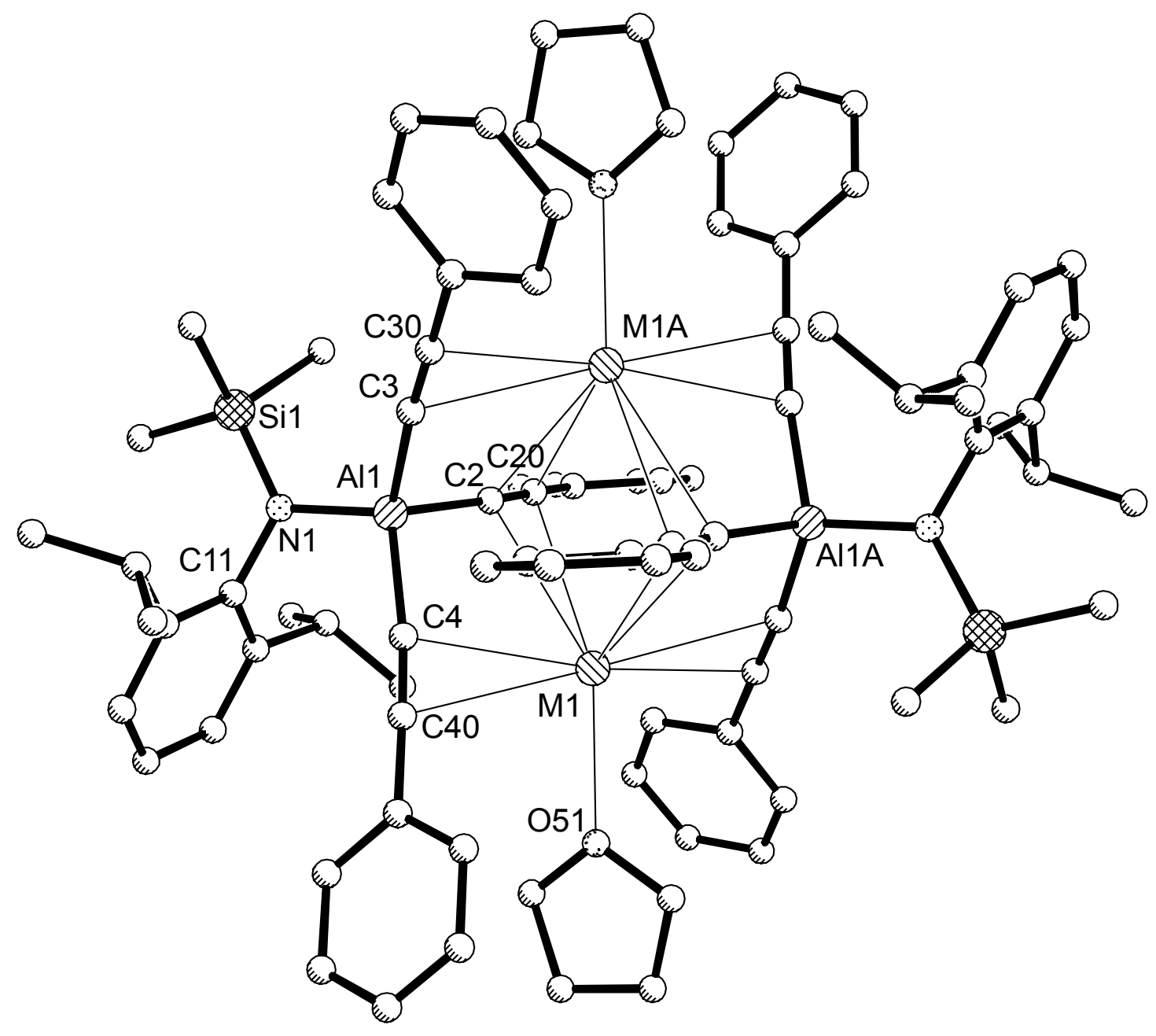

$\mathrm{M}=\mathrm{K}^{+}$(4); $\mathrm{Na}^{+}$(5) 
Bei Einsatz eines Lithium-Ethinyl-Salzes erhält man einen öligen Rückstand, der mit Dioxan versetzt als Verbindung 6 charakterisiert werden konnte.

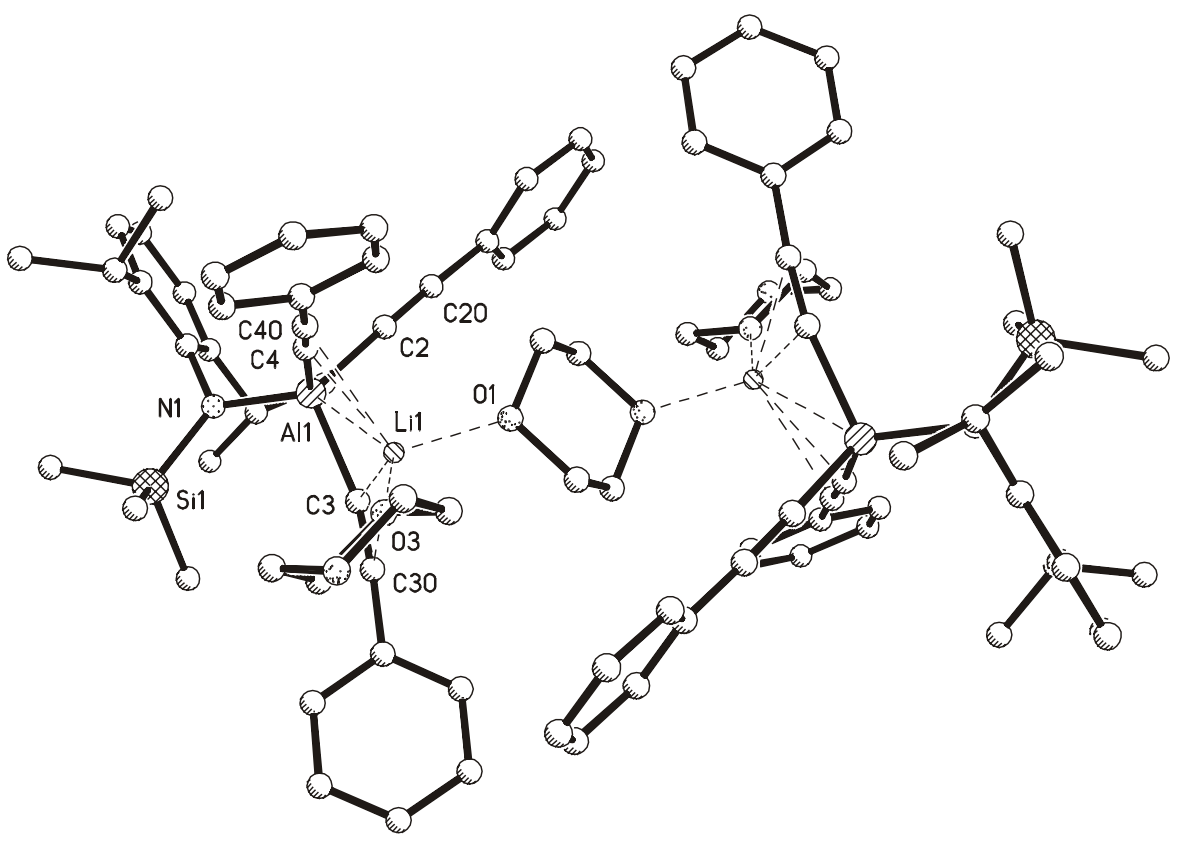

6

In 6 sind die monomeren Einheiten über die Sauerstoffatome des Dioxan-Moleküls verbrückt. Die dreifache Substitution mit terminalen Ethinylgruppen an dem Aluminiumatom findet auch bei Einsatz von sterisch sehr anspruchsvollen $\mathrm{SiMe}_{3}$-Gruppen am Ethinyl-Liganden statt. So entsteht bei der Reaktion von 3 mit drei Äquivalenten $\mathrm{LiC} \equiv \mathrm{CSiMe}_{3}$ die als Monomer vorliegende Verbindung 7.

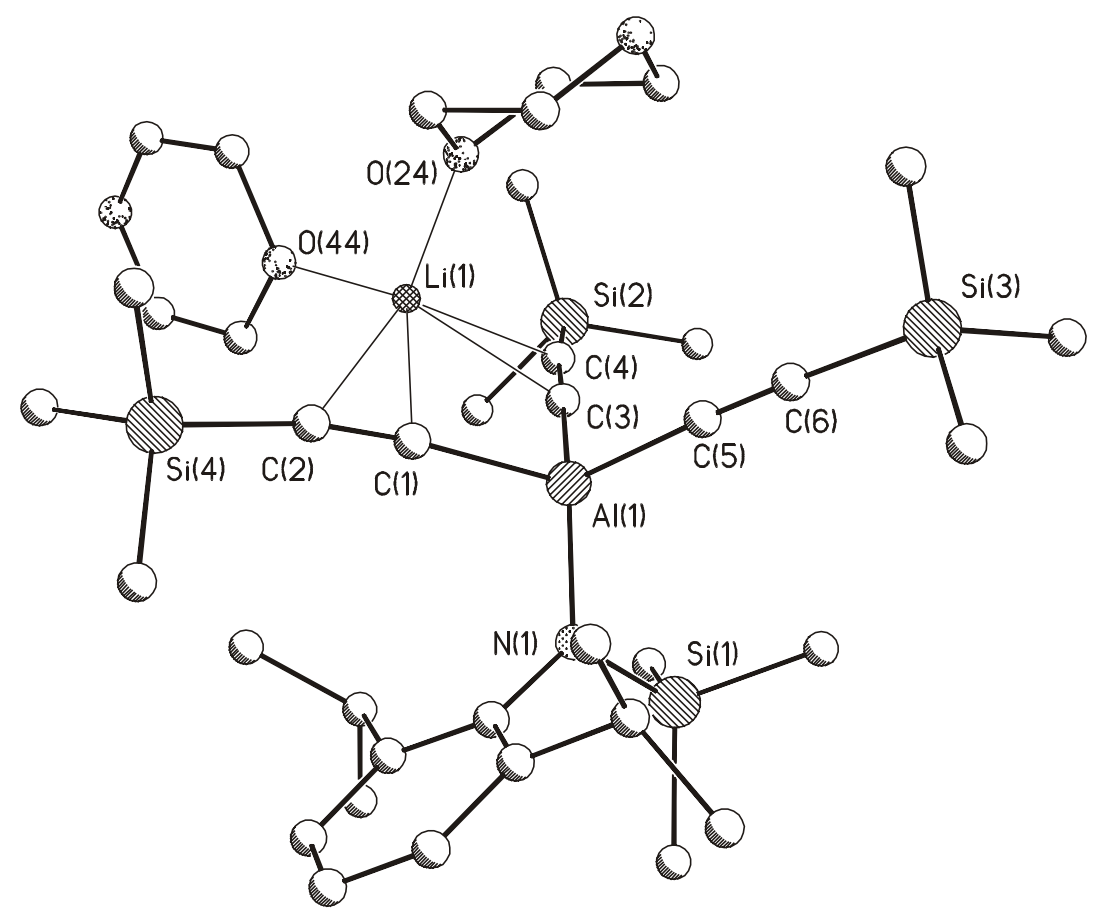

7

In 7 ist das Lithium-Kation ebenfalls an die Ethinyl-Liganden koordiniert. 
Für weitere Untersuchungen des Aufbaus des Verbindungstyps $\mathrm{Li}\left[\mathrm{RM}\left(\mathrm{C} \equiv \mathrm{CSiMe}_{3}\right)_{3}\right]$ $\left(\mathrm{R}=2,6-i \mathrm{Pr}_{2} \mathrm{C}_{6} \mathrm{H}_{3} \mathrm{~N}\left(\mathrm{SiMe}_{3}\right) ; \mathrm{M}=\mathrm{Al}, \mathrm{Ga}, \mathrm{In}\right)$ wurde das Zentralatom variiert. Hierfür wurde als Ausgangsverbindung [ $\left.\mathrm{Li} \cdot \mathrm{Et}_{2} \mathrm{O}\left(2,6-i \mathrm{Pr}_{2} \mathrm{C}_{6} \mathrm{H}_{3} \mathrm{~N}\left(\mathrm{SiMe}_{3}\right) \mathrm{GaCl}_{3}\right)\right]$ (8) dargestellt.

Es entstand die erste ionogene Galliumverbindung mit drei terminalen Ethinyl-Liganden (9).

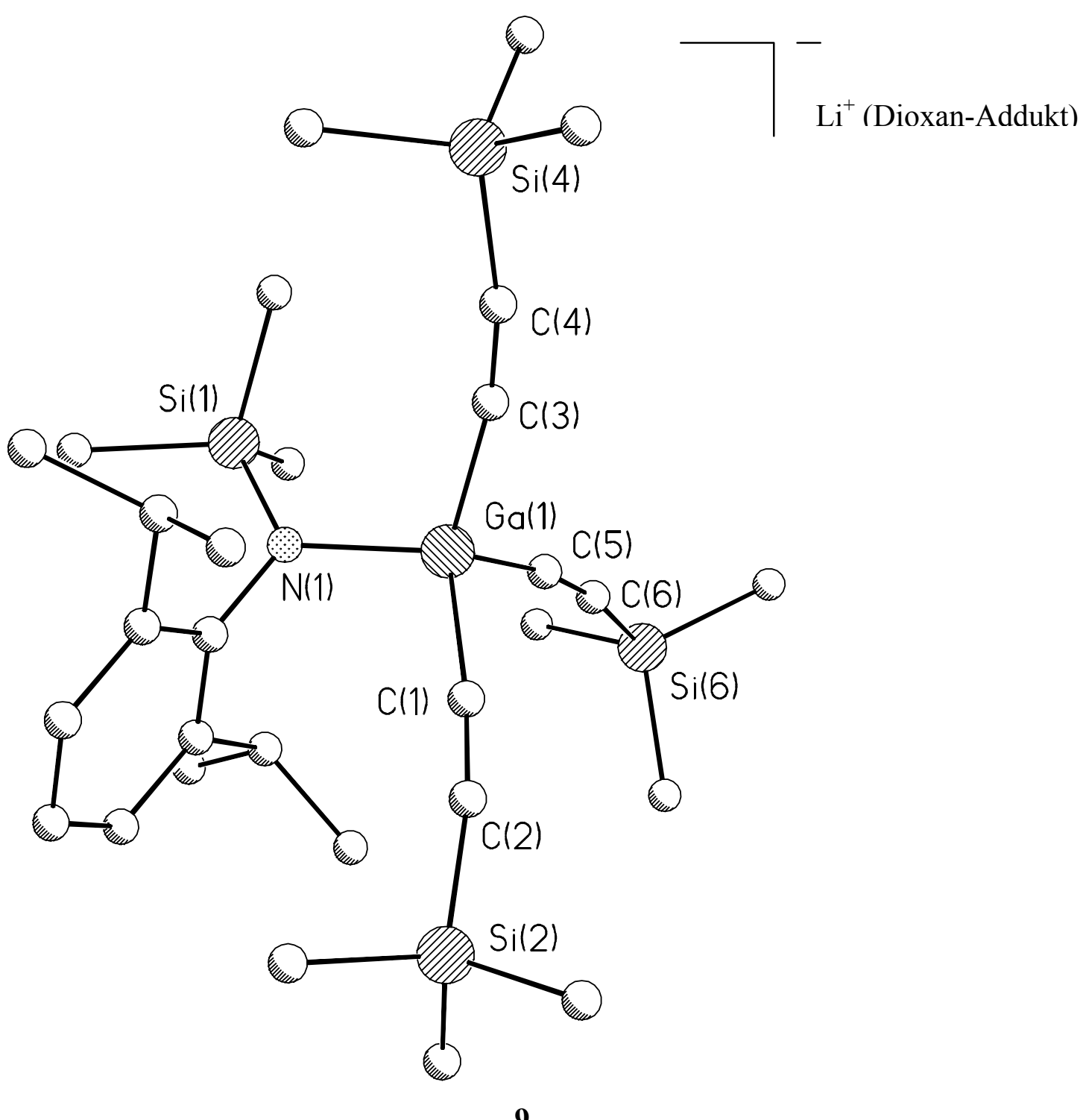

9

Im Unterschied zu 4, 5, 6 und 7 ist in 9 das Lithium-Kation von Dioxan-Molekülen koordiniert und die Ga-C $\equiv \mathrm{C}-$ Winkel sind deutlich kleiner als $180^{\circ}$. 
Für die Darstellung der ersten ionogenen Indiumverbindung mit drei terminalen EthinylLiganden wurde $\left[\mathrm{Li} \cdot 3\right.$ THF $\left.\left(2,6-i \mathrm{Pr}_{2} \mathrm{C}_{6} \mathrm{H}_{3} \mathrm{~N}\left(\mathrm{SiMe}_{3}\right) \mathrm{InCl}_{3}\right)\right]$ mit drei Äquivalenten $\mathrm{LiC} \equiv \mathrm{CSiMe}_{3}$ umgesetzt. Kristallisation aus Dioxan lieferte $\mathbf{1 0}$ in Form farbloser Kristalle.

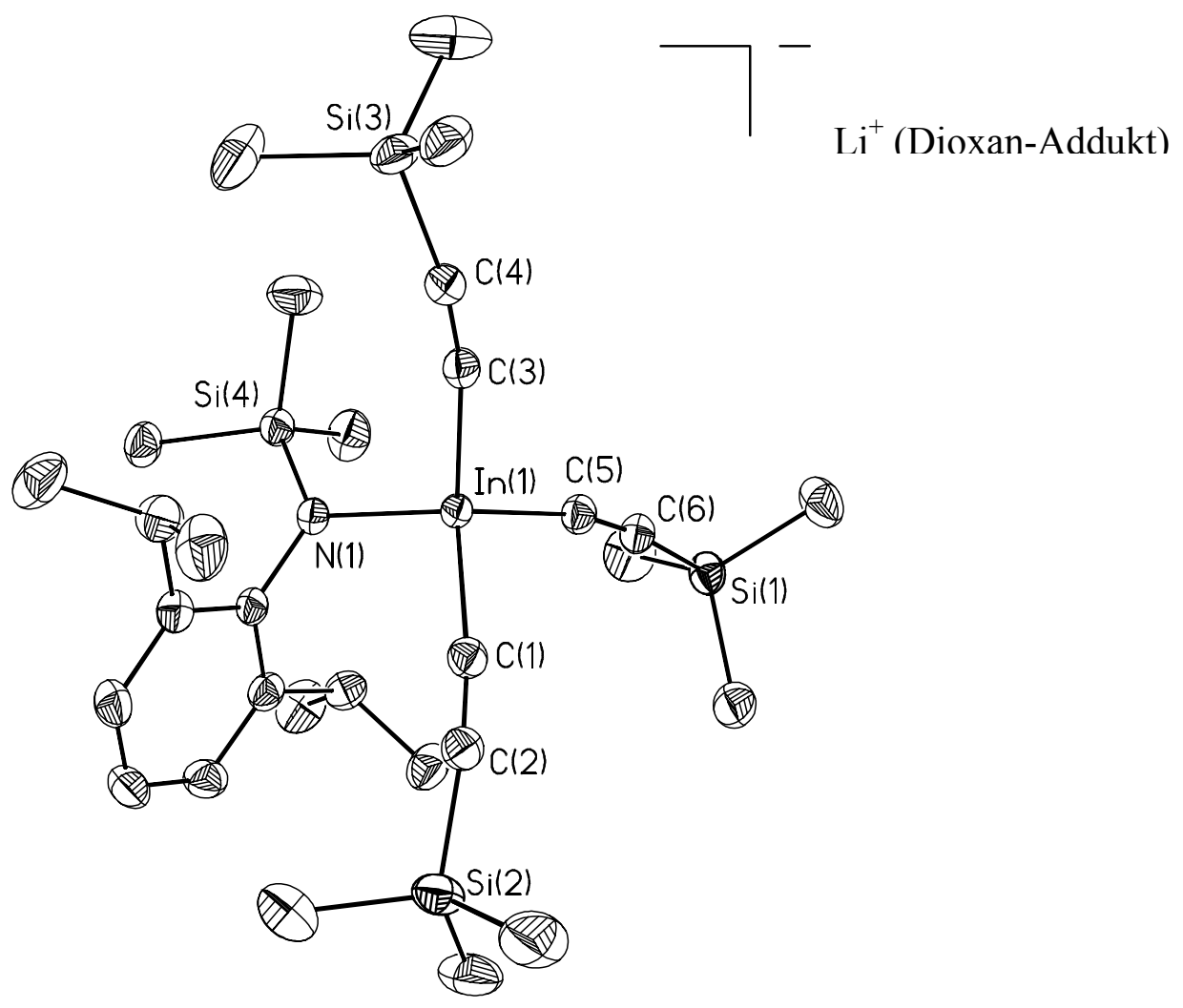

10

Wie in Verbindung 9 ist das Lithium-Kation an Lösungsmittel-Moleküle koordiniert.

Wird die Reaktion hingegen in einem Lösungsmittelgemisch aus THF und Toluol durchgeführt, so kann 11 mit einem $\pi$-koordinierten Li-Kation isoliert werden.

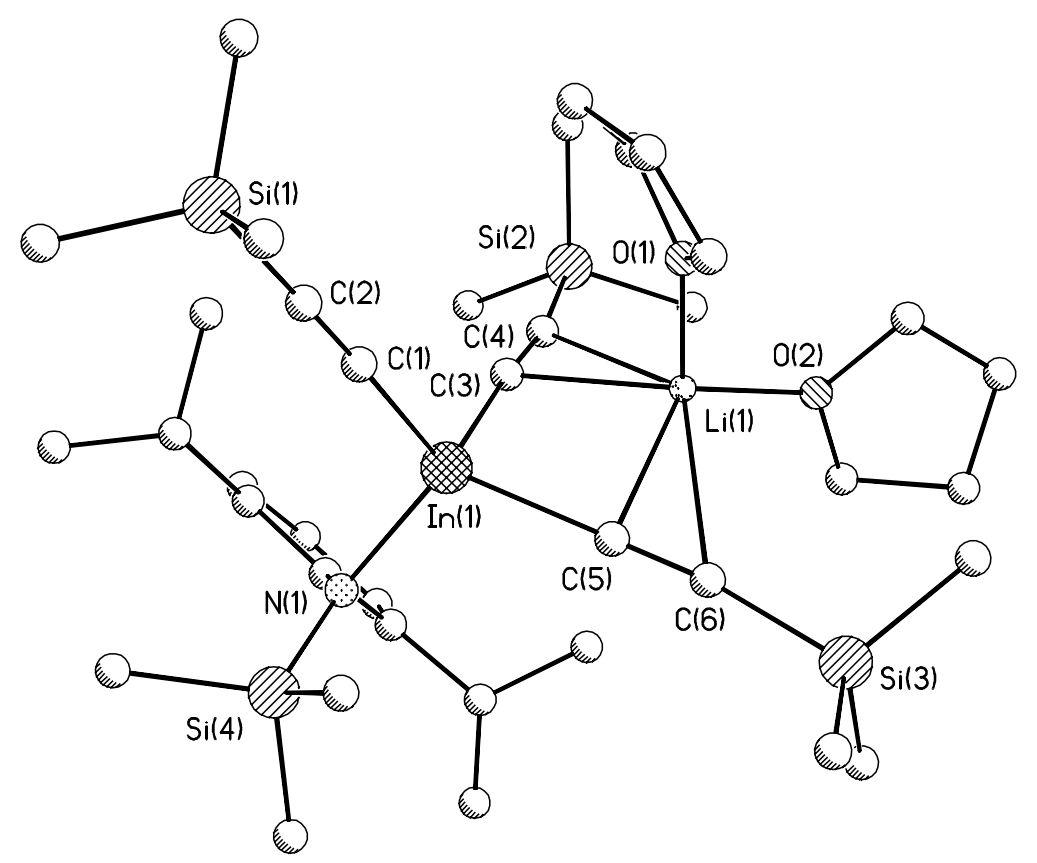


Da die Ethinyl-Chemie der 13. Gruppe des Periodensystems trotz ihrer großen Bedeutung in der organischen Synthese bisher kaum untersucht wurde, wurden umfangreiche Experimente durchgeführt. Dabei konnten mit 12, 13 und 14 die ersten Diethinyl-Halogen-Metallane der 13. Gruppe des Periodensystems synthetisiert und strukturell charakterisiert werden. In diesen Verbindungen fungiert tert.-Butylethinyl als Ligandensystem.

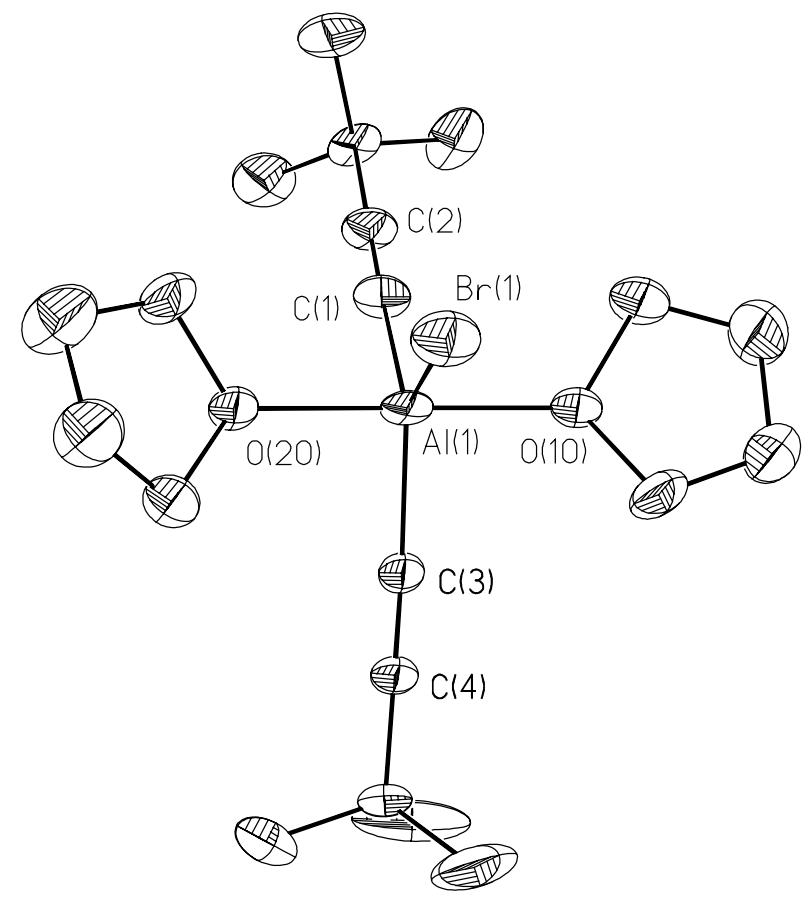

12

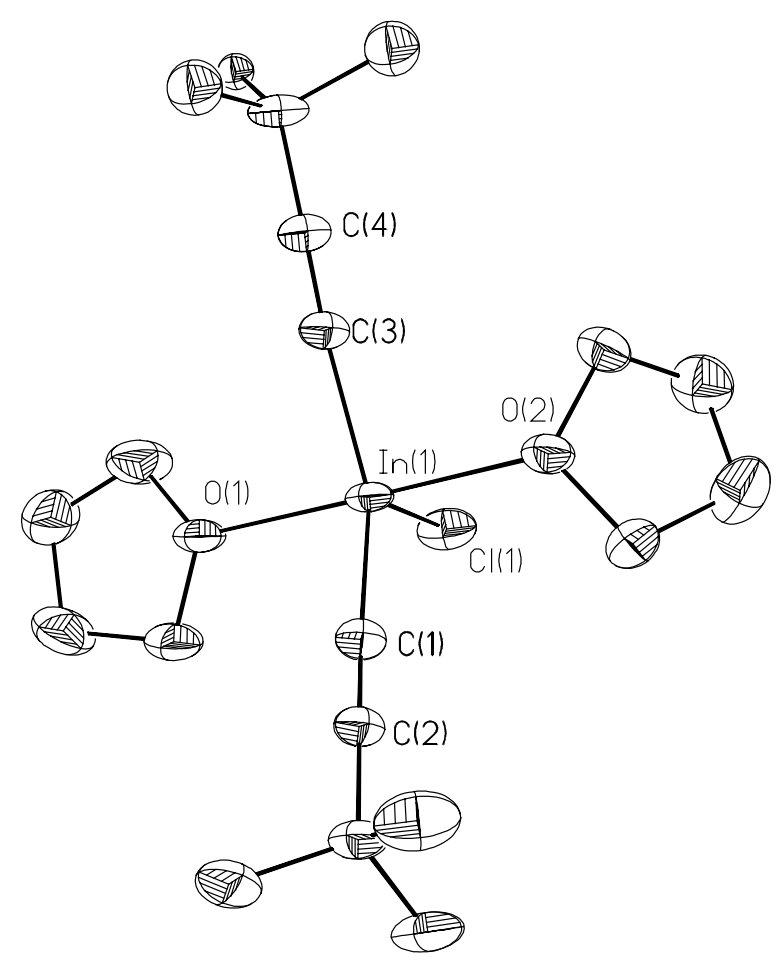

14

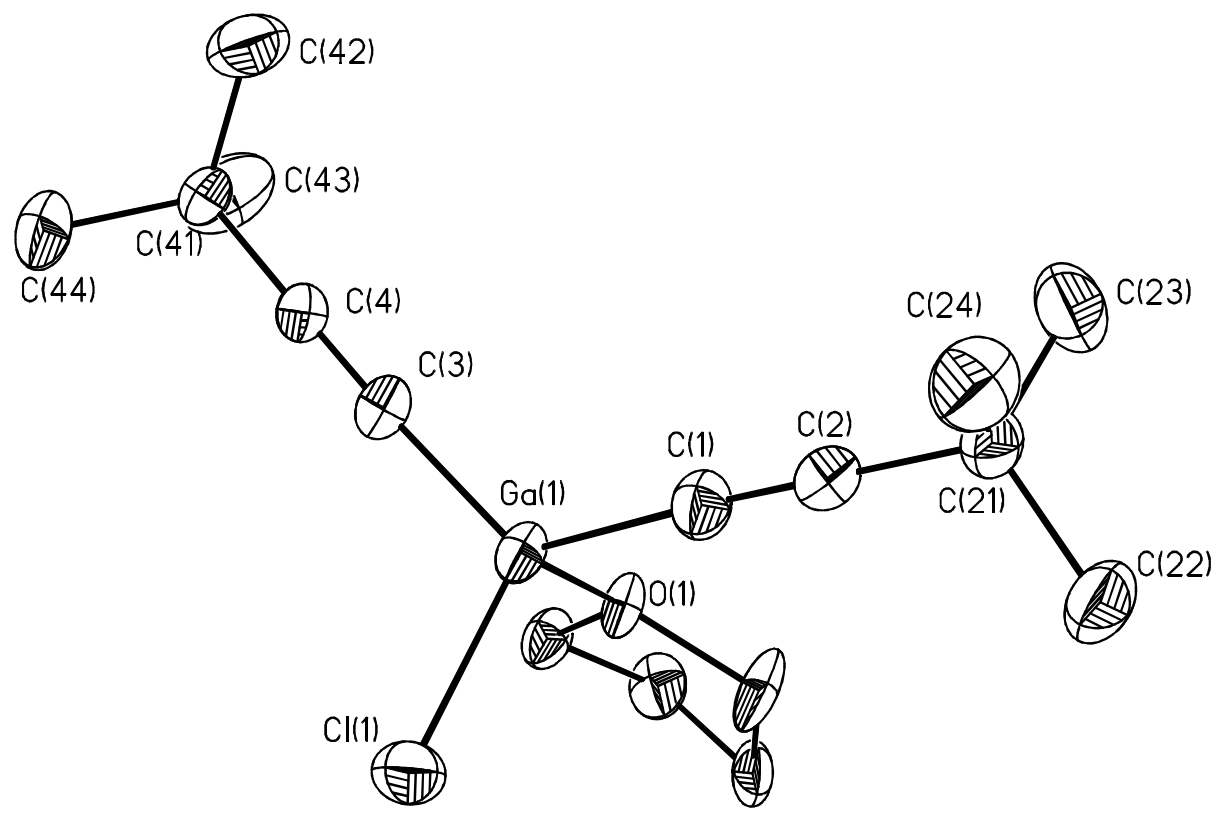

13

Bei 13 handelt es sich um die erste Galliumverbindung mit zwei terminalen Ethinyl-Einheiten. 
Die Erwartung, mit den Verbindungen 12, 13 oder 14 gezielte Reaktionen durchführen zu können, wurde nicht erfüllt. Bei der Reaktion von 12, 13 und 14 mit einem Organo-Lithiumsalz konnte nur beim Einsatz von 13 die anionische Galliumverbindung 15, bei der das Gallium außer zu dem 2,6-i $\operatorname{Pr}_{2} \mathrm{C}_{6} \mathrm{H}_{3} \mathrm{~N}\left(\mathrm{SiMe}_{3}\right)$-Liganden zu drei terminalen Ethinylgruppen gebunden ist, isoliert werden. In $\mathbf{1 5}$ ist das Lithium-Kation außer an zwei THF-Moleküle auch an zwei Ethinylgruppen des Anions koordiniert.

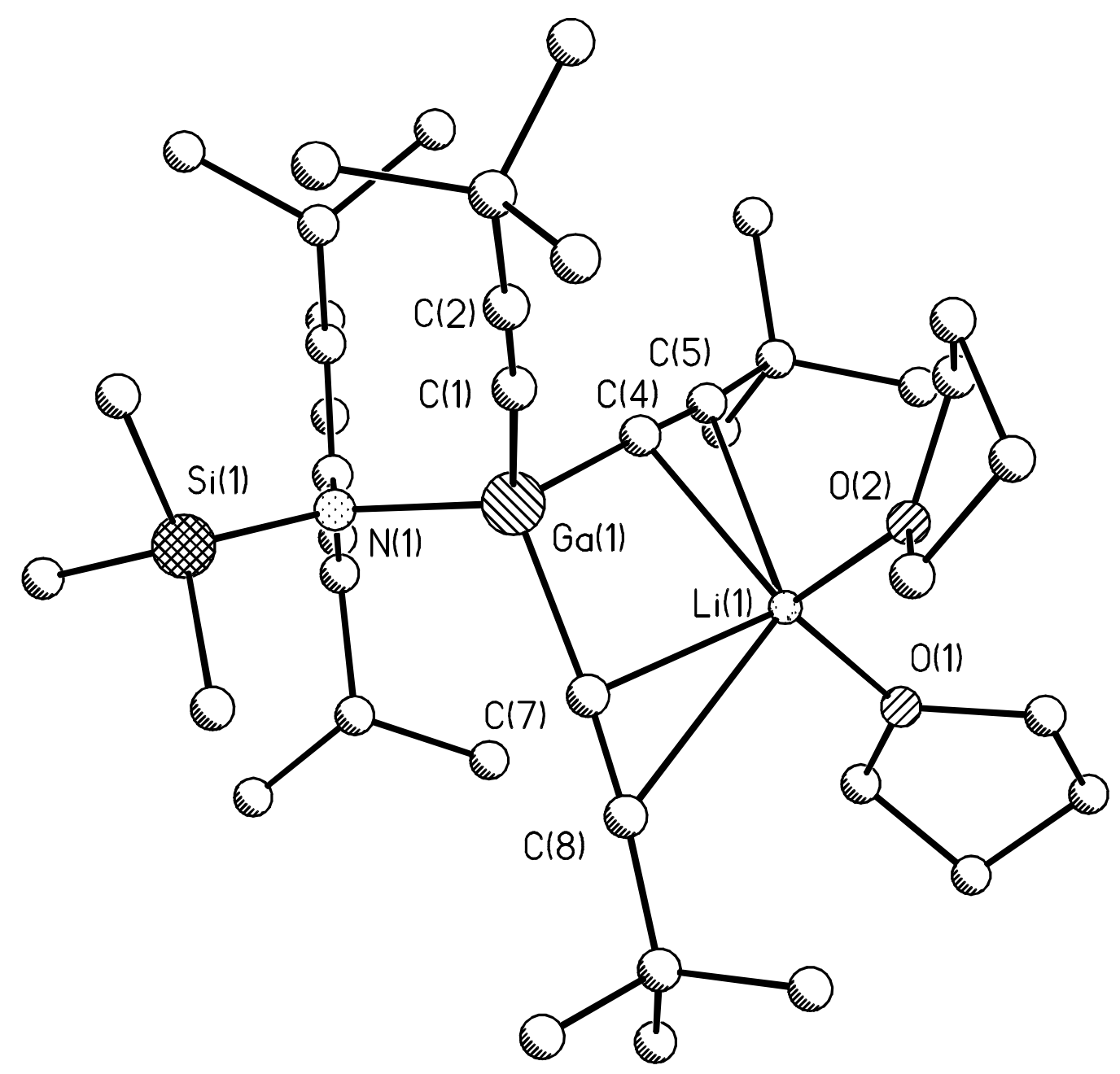


Bei der Reaktion von Triel-trihalogeniden mit drei Äquivalenten $\mathrm{LiC} \equiv \mathrm{C} t \mathrm{Bu}$ sind die Triethinylmetallane $16(\mathrm{Al}), 17(\mathrm{Ga})$ und 18 (In) in guter Ausbeute erhältlich. Bei 17 handelt es sich um das erste strukturell charakterisierte Triethinylgallan.

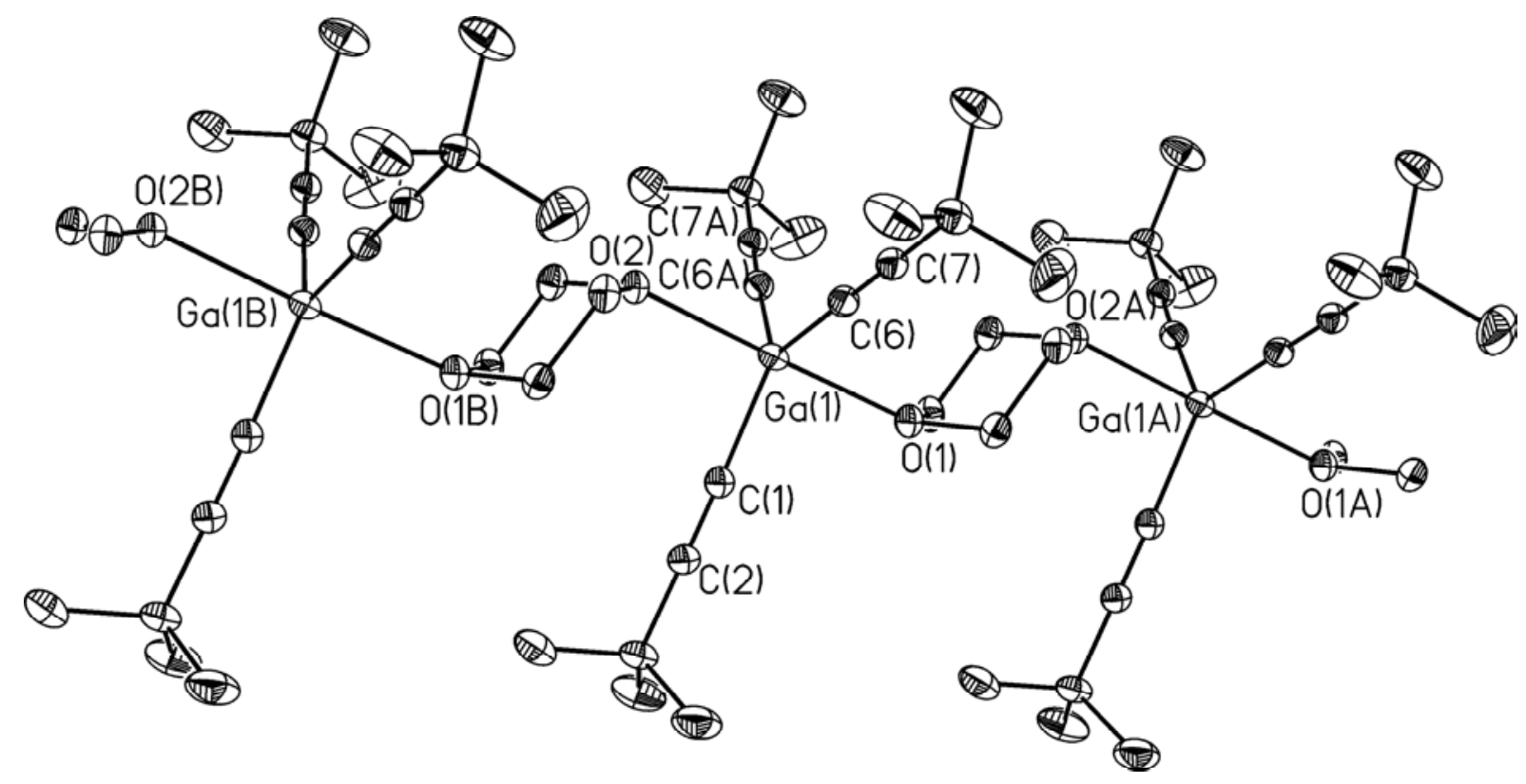

17

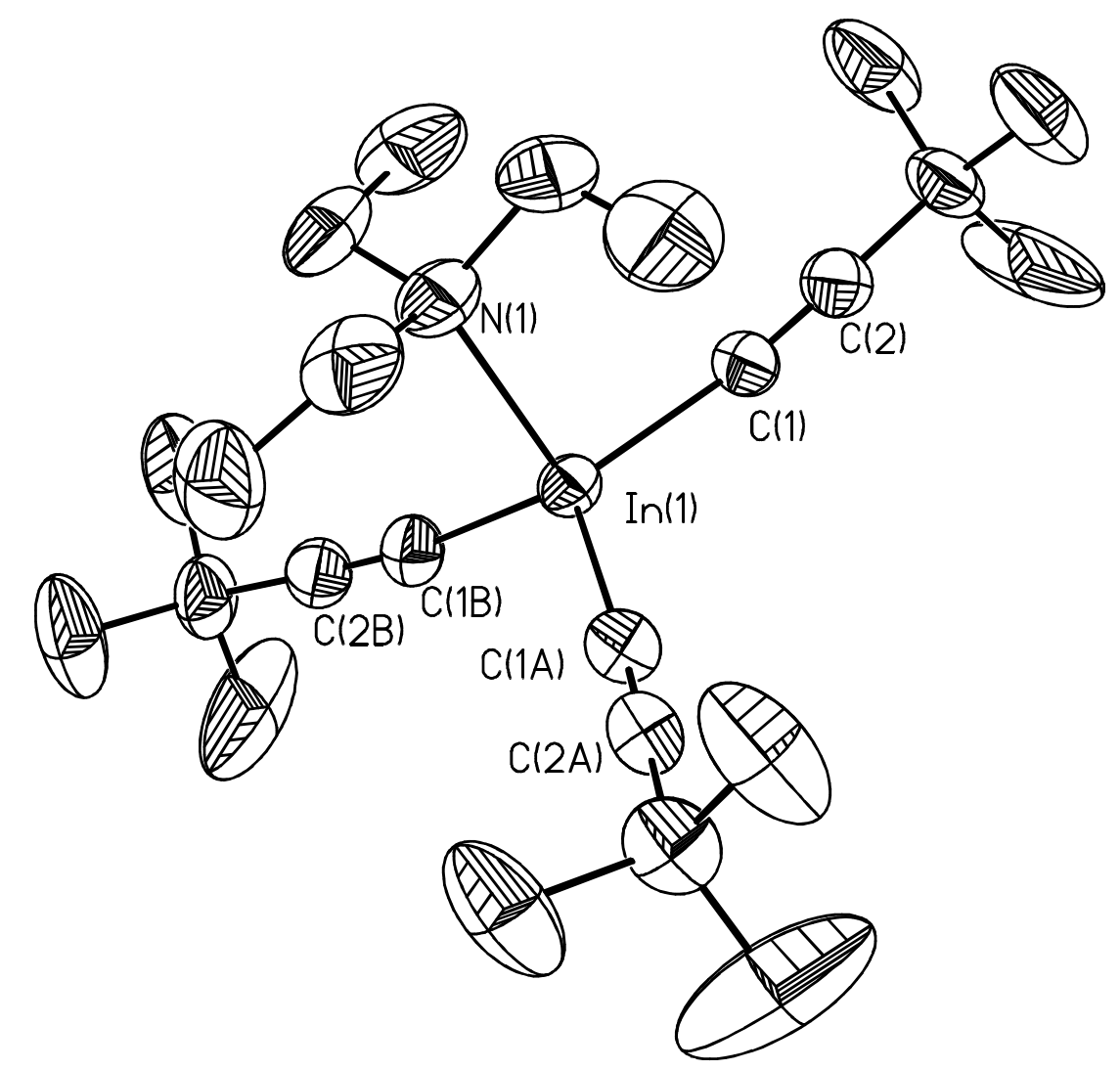

18

Die Reaktion von $\mathrm{LiAlH}_{4}$ mit $\mathrm{H}-\mathrm{C} \equiv \mathrm{C} t \mathrm{Bu}$ führt zur Bildung von $19\left(\left[\mathrm{Li} \cdot \mathrm{THF}\left(\mathrm{Al}(\mathrm{C} \equiv \mathrm{C} t \mathrm{Bu})_{4}\right)\right]\right.$. 
Tabelle 8: Übersicht über charakteristische Daten von in dieser Arbeit vorgestellten MetallEthinyl-Verbindungen:

\begin{tabular}{|c|c|c|c|c|c|}
\hline \multirow[t]{2}{*}{ Verbindung } & \multirow{2}{*}{$\begin{array}{l}\text { M-C }_{\text {Alkinyl}^{-}} \\
\text {Abstand }(\AA)\end{array}$} & \multirow{2}{*}{$\begin{array}{l}\mathrm{C} \equiv \mathrm{C}-\mathrm{Abstand} \\
(\AA)\end{array}$} & \multirow{2}{*}{$\begin{array}{l}\mathrm{M}-\mathrm{C} \equiv \mathrm{C}- \\
\text { Bindungswinkel }\end{array}$} & \multicolumn{2}{|c|}{$\delta\left({ }^{13} \mathrm{C}-\mathrm{NMR}\right)$} \\
\hline & & & & $\mathrm{C}_{\alpha}$ & $\mathrm{C}_{\beta}$ \\
\hline 4 & $1.962-1.984$ & $1.205-1.212$ & $165.7^{\circ}-175.0^{\circ}$ & 113.5 & 106.1 \\
\hline 5 & \begin{tabular}{|l|}
$1.960-1.988$ \\
\end{tabular} & $1.203-1.213$ & $164.2^{\circ}-176.7^{\circ}$ & 112.5 & 105.4 \\
\hline 6 & $1.943-1.998$ & $1.209-1.210$ & $166.6^{\circ}-175.6^{\circ}$ & 109.0 & 108.8 \\
\hline 7 & \begin{tabular}{|l|}
$1.947-1.979$ \\
\end{tabular} & $1.205-1.215$ & $175.0^{\circ}-177.3^{\circ}$ & 109.0 & 95.3 \\
\hline 9 & \begin{tabular}{|l|}
$1.966-1.971$ \\
\end{tabular} & $1.205-1.207$ & $169.9^{\circ}-173.7^{\circ}$ & 107.3 & 95.2 \\
\hline 10 & \begin{tabular}{|l|}
$2.148-2.217$ \\
\end{tabular} & 1.206-1.210 & $168.1^{\circ}-170.9^{\circ}$ & 114.1 & 95.2 \\
\hline 11 & - & - & - & 111.7 & 95.2 \\
\hline 12 & \begin{tabular}{|l|}
$1.934-1.937$ \\
\end{tabular} & $1.190-1.205$ & $177.1^{\circ}$ & 67.9 & 92.6 \\
\hline 13 & $1.908-1.911$ & \begin{tabular}{|l|}
$1.196-1.198$ \\
\end{tabular} & $175.3^{\circ}-175.5^{\circ}$ & 83.7 & 116.4 \\
\hline 14 & $2.101-2.104$ & $1.196-1.197$ & $175.9^{\circ}-177.6^{\circ}$ & 88.3 & 118.2 \\
\hline 15 & \begin{tabular}{|l}
$1.956-1.991$ \\
\end{tabular} & \begin{tabular}{|l}
$1.198-1.203$ \\
\end{tabular} & $174.7-176.2$ & - & - \\
\hline 16 & - & - & - & 94.1 & 112.6 \\
\hline 17 & $1.933-1.934$ & \begin{tabular}{|l|}
$1.205-1.209$ \\
\end{tabular} & $178.0^{\circ}-178.5^{\circ}$ & 86.3 & 116.5 \\
\hline 18 & 2.116 & 1.201 & $172.3^{\circ}$ & 90.3 & 119.2 \\
\hline 19 & - & - & - & 96.3 & 113.7 \\
\hline
\end{tabular}

Im Vergleich aller ionogenen Ethinylverbindungen (4-11) miteinander findet man, dass die chemische Verschiebung im ${ }^{13} \mathrm{C}$-NMR-Spektrum der in $\alpha$-Position zum Metallatom gebundenen Kohlenstoffatome ähnlich groß ist. Im Gegensatz dazu wird die chemische Verschiebung der in $\beta$-Position gebundenen Kohlenstoffatome stärker von den Nachbargruppen beeinflusst. Der $\mathrm{M}-\mathrm{C} \equiv \mathrm{C}$-Bindungswinkel nimmt in vergleichbaren Verbindungen $\mathbf{( 7 , 9 , 1 0 )}$ in der Reihenfolge $\mathrm{Al}>\mathrm{Ga}>\mathrm{In} \mathrm{ab}$. 
Die Reaktion von $\mathrm{Cp}_{2} \mathrm{ZrMe}_{2}$ mit $16\left(\mathrm{Al}(\mathrm{C} \equiv \mathrm{C} t \mathrm{Bu})_{3} \cdot\right.$ Dioxan $)$ führt zur Bildung des DiethinylZirconocens 20, während bei dem Einsatz von 4, 19 oder [ $\left.\mathrm{Li} \cdot 2 \mathrm{THF}\left(\mathrm{Al}(\mathrm{C} \equiv \mathrm{CPh})_{4}\right)\right]$ keine Reaktion erfolgt.

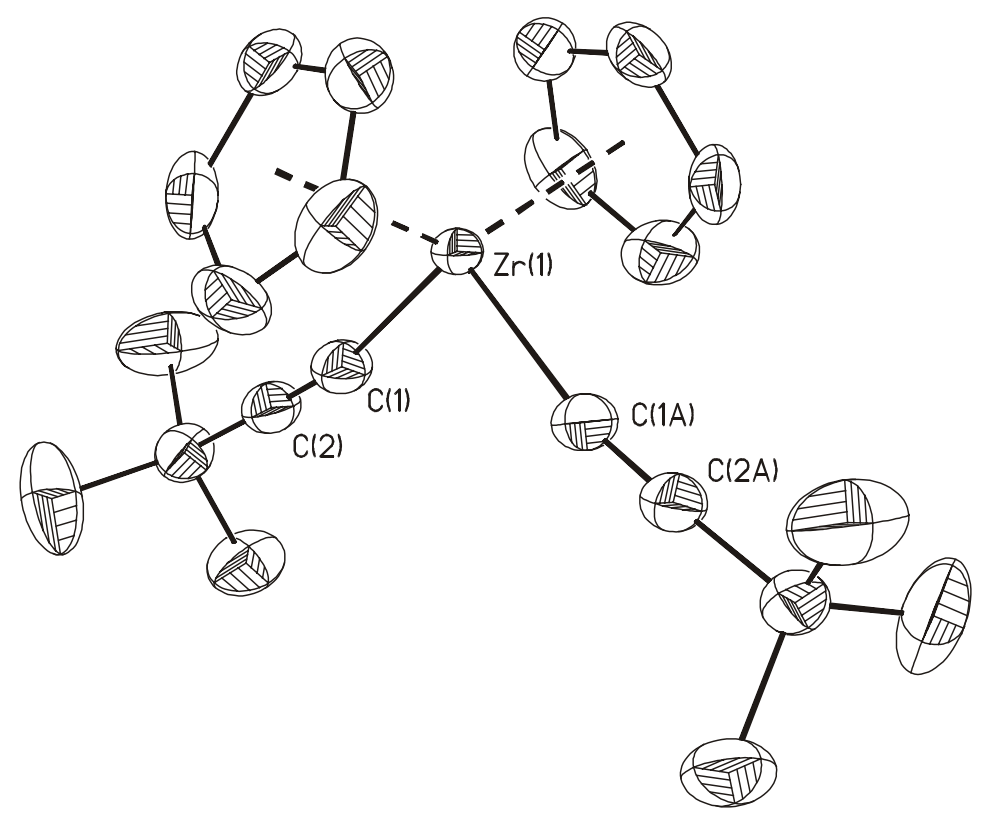

20

Neben der solvatfreien Aluminiumchlorid-Verbindung 3 wurde die erste solvatfreie, über Iodatome verbrückte dimere Aluminiumdiiodid-Verbindung 21 dargestellt.

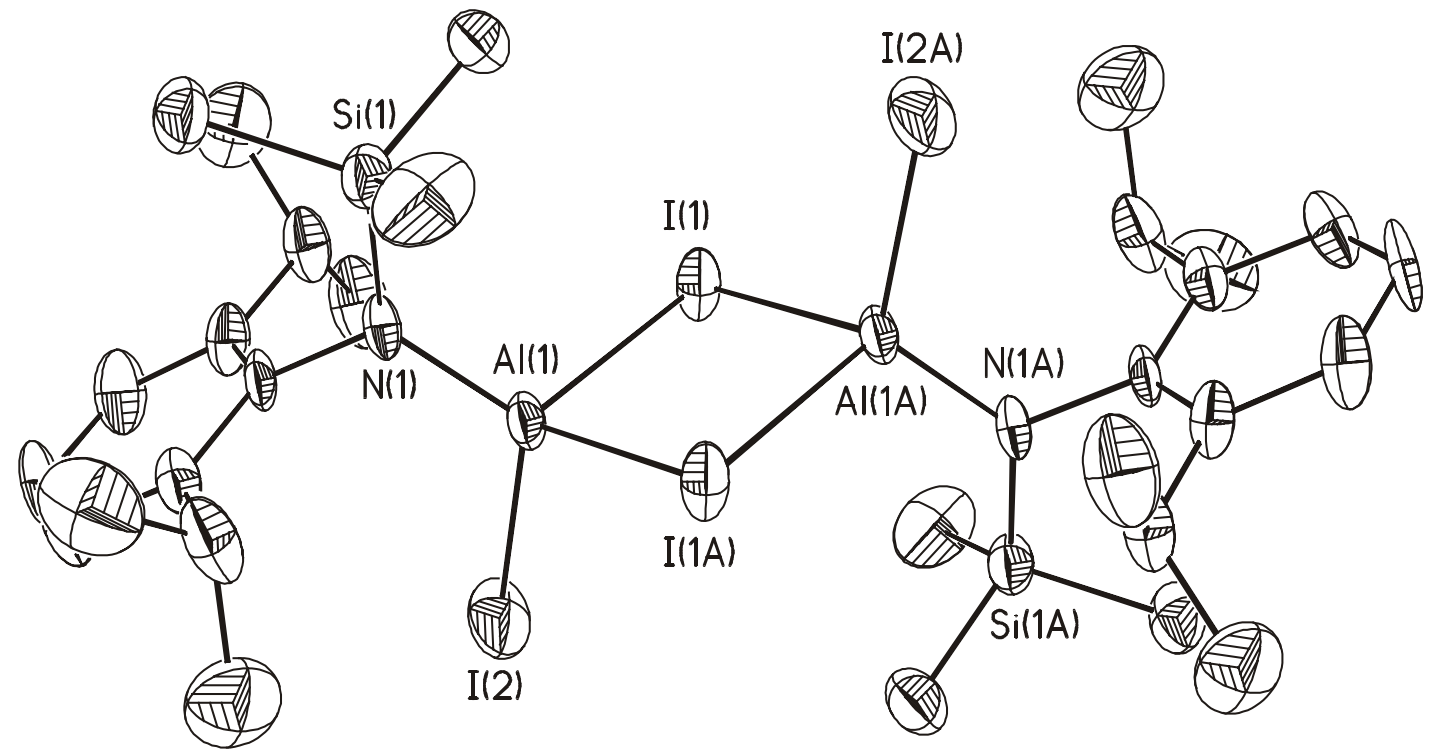


Die Reduktion von 21 mit Natrium- Kalium-Legierung liefert in guter Ausbeute die erste nur durch Stickstoffbasen-stabilisierte tetramere Aluminium(+I)-Verbindung 22.

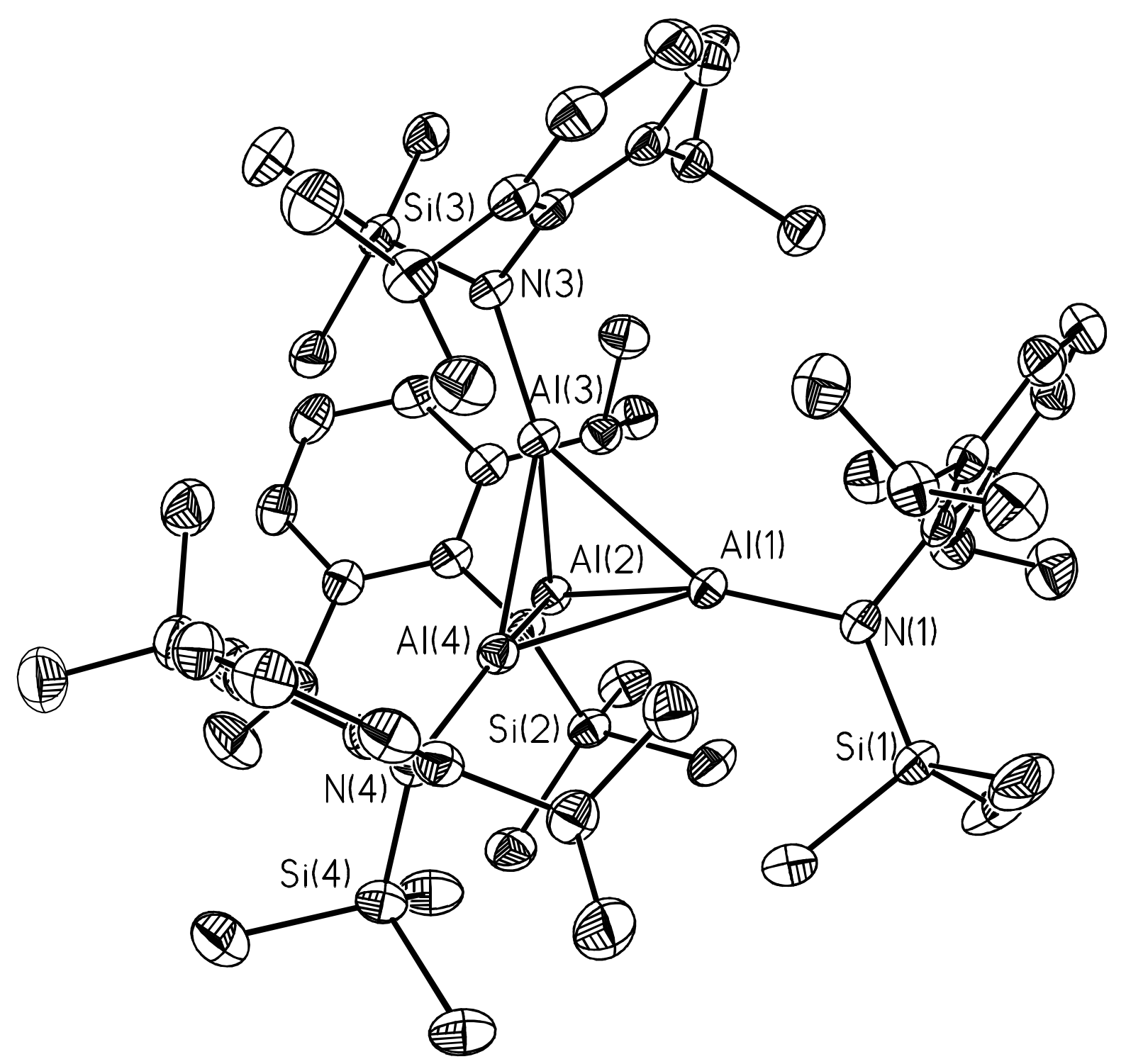




\section{Ausblick}

Im Rahmen dieser Arbeit ist es gelungen, eine Reihe neuer Verbindungen mit Elementen der 13. Gruppe des Periodensystems zu synthetisieren.

Durch die einfache Transformation der Alkinylgruppen unter milden Bedingungen sollten die in dieser Arbeit vorgestellten Produkte neue Anwendungsmöglichkeiten in der organischen Synthese bieten.

Nach Ausbeuteoptimierung bei der Darstellung von 22 sollte diese Verbindung aufgrund ihres hohen synthetischen Potentials eine gute Ausgangsverbindung zur Darstellung neuer Materialien sein. 


\section{Experimenteller Teil}

\subsection{Allgemeine Arbeitstechniken und Messmethoden}

Alle Versuche wurden in ausgeheizten und im Vakuum abgekühlten Glasgeräten unter einer getrockneten und sauerstofffreien Stickstoffatmosphäre durchgeführt. Die verwendeten Lösungsmittel wurden nach bekannten Methoden ${ }^{[1]}$ getrocknet und mit Stickstoff gesättigt.

Schmelz- und Zersetzungspunkte wurden in mit Wachs verschlossenen Glaskapillaren mit dem Gerät Büchi MeltingPoint B 540 bestimmt.

NMR-Spektren wurden auf den Geräten Bruker Avance 200 und 500, Bruker AM 250 und Bruker AS 400 bestimmt. Als externe Standards wurden TMS $\left({ }^{1} \mathrm{H}-,{ }^{13} \mathrm{C}-,{ }^{29} \mathrm{Si}-\mathrm{NMR}\right)$ und $\mathrm{LiCl}$ ( $\left.{ }^{7} \mathrm{Li}-\mathrm{NMR}\right) \quad$ verwendet. Heterokern-NMR-Spektren wurden ${ }^{1} \mathrm{H}$-breitbandentkoppelt aufgenommen. Verwendete Lösungsmittel und Betriebsfrequenzen sind bei den Versuchsbeschreibungen aufgeführt. Die Multiplizitäten sind wie folgt abgekürzt: $s=$ Singulett, $\mathrm{d}=$ Dublett, $\mathrm{t}=$ Triplett, $\mathrm{q}=$ Quartett, $\mathrm{sept}=$ Septett, $\mathrm{m}=$ Multiplett. Als Multipletts werden auch nicht aufgelöste Gruppen von Signalen geringerer Multiplizität bezeichnet. Die chemischen Verschiebungen $\delta$ werden in ppm angegeben, wobei negative Vorzeichen einer Verschiebung zu höheren Feldstärken und positive Vorzeichen einer Verschiebung zu tieferen Feldstärken bezogen auf den Standard entsprechen.

Massenspektren wurden auf den Geräten Finnigan MAT System 8230, Varian MAT CH5 und Finnigan MAT 95 aufgenommen. Es wurde die Elektronenstoß-Ionisation (EI) bei $70 \mathrm{eV}$ sowie die Methode des Fast-Atom-Bombardement als Ionisierungsmethode verwendet. Es wird der stärkste zu einer Isotopenverteilung gehörende Peak mit seiner relativen prozentualen Intensität angegeben.

IR-Spektren wurden auf einem Bio-Rad Digilab FTS-7 Spektrometer aufgenommen. Feststoffe wurden mit getrocknetem Nujol verrieben und zwischen KBr-Platten vermessen. Es werden nur mittelstarke (m), starke (s) und sehr starke (ss) sowie charakteristische Banden angegeben.

Elementaranalysen wurden vom analytischen Labor des Instituts für Anorganische Chemie der Universität Göttingen durchgeführt. 
Einkristallröntgenstrukturanalysen wurden auf einem Stoe-Siemens-AED-Vierkreisdiffraktometer oder einem Stoe-Siemens-Huber-Vierkreisdiffraktometer ausgerüstet mit einem Siemens-SMART-CCD-Flächenzähler an einem schockgekühlten Kristall im Öltropfen ${ }^{[103]}$ mit monochromatisierter Mo-K ${ }_{\alpha}$-Strahlung $(\lambda=0.71073 \AA)$ durchgeführt. Die Lösung der Strukturen erfolgte mit direkten Methoden (SHELX-97), ${ }^{[104]}$ die Verfeinerung gegen $F^{2}$ unter Verwendung aller Daten (SHELX-97). ${ }^{[105]}$ Alle Atome mit Ausnahme der Wasserstoffatome wurden anisotrop verfeinert. Die Positionierung der Wasserstoffatome erfolgte nach dem Reitermodell.

\subsection{Darstellung der Ausgangsverbindungen}

Die Ausgangsverbindungen waren mit Ausnahme von 2,6-i $\mathrm{Pr}_{2} \mathrm{C}_{6} \mathrm{H}_{3} \mathrm{~N}\left(\mathrm{SiMe}_{3}\right) \mathrm{Li}$ (vgl. 4.2.1) im Handel erhältlich oder wurden nach folgenden Literaturvorschriften hergestellt:

$$
\begin{aligned}
& {\left[2,6-i \mathrm{Pr}_{2} \mathrm{C}_{6} \mathrm{H}_{3} \mathrm{~N}\left(\mathrm{SiMe}_{3}\right) \mathrm{AlMe}_{2}\right]_{2},{ }^{[106]} \quad\left[\mathrm{Li} \cdot 3 \mathrm{THF}\left(2,6-i \mathrm{Pr}_{2} \mathrm{C}_{6} \mathrm{H}_{3} \mathrm{~N}\left(\mathrm{SiMe}_{3}\right) \mathrm{InCl}_{3}\right)\right],{ }^{[56]}} \\
& \mathrm{Cp}_{2} \mathrm{Ti}(\mathrm{C} \equiv \mathrm{CPh})_{2},{ }^{[28,107,108]} \quad \mathrm{Cp}_{2} \mathrm{Ti}(\mathrm{C} \equiv \mathrm{Ct} \mathrm{Bu})_{2},{ }^{[108]} \quad \mathrm{Cp}_{2} \mathrm{ZrMe}_{2}{ }^{[35]} \\
& {\left[\left\{\mathrm{N}(\mathrm{SiMe}) \mathrm{C}(\mathrm{Ph}) \mathrm{C}\left(\mathrm{SiMe}_{3}\right)_{2}\right\} \mathrm{AlH}(\mu-\mathrm{H})\right]_{2},{ }^{[17]}} \\
& \mathrm{Cp}_{2} \mathrm{Zr}(\mathrm{C} \equiv \mathrm{CPh})_{2}{ }^{[79]}, \quad \mathrm{Cp}_{2}{ }_{2} \mathrm{ZrCl}_{2},{ }^{[109,110]} \quad \mathrm{Cp}_{2} \mathrm{TiCl}_{2},{ }^{[35]}
\end{aligned}
$$

\subsubsection{Darstellung von 2,6-i $\mathrm{Pr}_{2} \mathrm{C}_{6} \mathrm{H}_{3} \mathrm{~N}\left(\mathrm{SiMe}_{3}\right) \mathrm{Li}$}

$\mathrm{Zu} 24.95 \mathrm{~g}$ (100 mmol) 2,6-i $\mathrm{Pr}_{2} \mathrm{C}_{6} \mathrm{H}_{3} \mathrm{~N}\left(\mathrm{SiMe}_{3}\right) \mathrm{H}$ in Toluol wurden bei $-78{ }^{\circ} \mathrm{C} 40 \mathrm{~mL}(100$ mmol) einer $2.5 \mathrm{M}$ Lösung von $n$-BuLi in Hexan langsam zugetropft. Das Gemisch wurde auf RT erwärmt und $4 \mathrm{~h}$ gerührt. Der entstandene weiße Niederschlag wurde abfiltriert und mit Hexan $(50 \mathrm{~mL})$ gewaschen. Man erhielt $18.13 \mathrm{~g}$ (71\%) eines weißen Feststoffs.

${ }^{1} \mathrm{H}-\mathrm{NMR}\left(200.13 \mathrm{MHz}, \mathrm{THF}-\mathrm{D}_{8}\right): \delta 6.80-6.20$ (m, $\left.3 \mathrm{H}, \mathrm{Ar} H\right), 4.06$ (sept, $J=7.0 \mathrm{~Hz}, 2 \mathrm{H}$, $\left.\mathrm{CH}\left(\mathrm{CH}_{3}\right)_{2}\right), 1.06\left(\mathrm{~d}, J=7.0 \mathrm{~Hz}, 12 \mathrm{H}, \mathrm{CH}\left(\mathrm{CH}_{3}\right)_{2}\right),-0.08\left(\mathrm{~s}, 9 \mathrm{H}, \mathrm{Si}\left(\mathrm{CH}_{3}\right)_{3}\right)$;

${ }^{7} \mathrm{Li}-\mathrm{NMR}\left(97.2 \mathrm{MHz}, \mathrm{THF}-\mathrm{D}_{8}\right): \delta-0.14$;

${ }^{13} \mathrm{C}$ NMR (125 MHz, THF-D 8 ): $\delta 158.3,143.8,122.3,114.8$ (s, arom. C), 27.0 (s, CH), 25.3 $\left(\mathrm{s}, \mathrm{CH}_{3}\right)$;

MS(EI): $m / z(\%): 248(M-\mathrm{Li}, 100), 233\left(M-\mathrm{Li}-\mathrm{CH}_{3}, 95\right)$;

IR (Nujol): $\tilde{v}=3059,3041,1587,1419,1384,1366,1307,1250,1236,1190,1106,1035$, 936, 828, 769, 742, 659, 613, 571, 533, 468, $444 \mathrm{~cm}^{-1}$;

EA für $\mathrm{C}_{15} \mathrm{H}_{26} \operatorname{LiNSi}(255.40)$ : gef.: C 71.30, H 10.15, N 5.36; ber.: C 70.5, H 10.3, N 5.5 \%. 


\subsection{Darstellung eines Aminoalans und dessen Umsetzung mit THF}

\subsubsection{Darstellung von $\left[\mathrm{Li} \cdot \mathrm{Et}_{2} \mathrm{O}\left(2,6-i \mathrm{Pr}_{2} \mathrm{C}_{6} \mathrm{H}_{3} \mathrm{~N}\left(\mathrm{SiMe}_{3}\right) \mathrm{AlH}_{3}\right)\right]_{2}$ (1)}

$\mathrm{Zu}$ einer Lösung von $2.50 \mathrm{~g}(10 \mathrm{mmol}) 2,6-i \mathrm{Pr}_{2} \mathrm{C}_{6} \mathrm{H}_{3} \mathrm{~N}\left(\mathrm{SiMe}_{3}\right) \mathrm{H}$ in Diethylether $(200 \mathrm{~mL})$ wurde bei $-20^{\circ} \mathrm{C}$ ein Überschuss ( 3 Äq; $1.14 \mathrm{~g} ; 30 \mathrm{mmol}$ ) $\mathrm{LiAlH}_{4}$ gegeben und 5 Tage lang bei RT gerührt. Nach Filtration über Celite wurde das Lösungsmittel im Vakuum entfernt. Der farblose, geleeartige Rückstand wurde mit Hexan versetzt $(20 \mathrm{~mL})$ und über Nacht bei $-32{ }^{\circ} \mathrm{C}$ gelagert. Der entstandene Niederschlag wurde in der Kälte abfiltriert und mit wenig kaltem Hexan gewaschen. Man erhielt $2.27 \mathrm{~g}(63 \% \mathrm{~d}$. Th.) eines farblosen Rückstands.

Zers.: $130^{\circ} \mathrm{C}$;

${ }^{1} \mathrm{H}-\mathrm{NMR}\left(200.13 \mathrm{MHz}, \mathrm{C}_{6} \mathrm{D}_{6}\right): \delta 7.20-6.90(\mathrm{~m}, 3 \mathrm{H}, \mathrm{Ar} H), 3.90$ (sept, $J=7.0 \mathrm{~Hz}, 2 \mathrm{H}$, $\left.\mathrm{CH}\left(\mathrm{CH}_{3}\right)_{2}\right), 3.19$ (q, $\left.J=7.0 \mathrm{~Hz}, 4 \mathrm{H}, \mathrm{O}-\mathrm{CH}_{2}\right), 1.40\left(\mathrm{~d}, J=7.0 \mathrm{~Hz}, 6 \mathrm{H}, \mathrm{CH}\left(\mathrm{CH}_{3}\right)_{2}\right), 1.31$ (d, $\left.J=7.0 \mathrm{~Hz}, 6 \mathrm{H}, \mathrm{CH}\left(\mathrm{CH}_{3}\right)_{2}\right), 0.96\left(\mathrm{t}, J=7.0 \mathrm{~Hz}, 6 \mathrm{H}, \mathrm{OCH}_{2}-\mathrm{CH}_{3}\right), 0.28\left(\mathrm{~s}, 9 \mathrm{H}, \mathrm{Si}\left(\mathrm{CH}_{3}\right)_{3}\right)$;

${ }^{7} \mathrm{Li}-\mathrm{NMR}\left(97.2 \mathrm{MHz}, \mathrm{C}_{6} \mathrm{D}_{6}\right): \delta$-0.46;

MS(EI): $m / z: 276\left(1 / 2\left[M^{+}-2\right.\right.$ H- Et $\left.\left.2 \mathrm{O}-\mathrm{Li}^{+}\right]\right), 249\left(1 / 2\left[M^{+}-\mathrm{AlH}_{3}-\mathrm{Et}_{2} \mathrm{O}-\mathrm{Li}^{+}\right]\right), 234\left[M^{+}-\mathrm{AlH}_{3^{-}}\right.$ $\left.\mathrm{Et}_{2} \mathrm{O}-\mathrm{Li}^{+}-\mathrm{Me}\right]$

IR (Nujol): $\widetilde{v}=3048,1773,1433,1382,1361,1316,1243,1188,1154,1106,1063,1042$, 923, 829, 743, 680, 633, 544, $437 \mathrm{~cm}^{-1}$;

EA für $\mathrm{C}_{19} \mathrm{H}_{39} \mathrm{AlLiONSi}$ (359.53): gef.: C 64.17, H 10.62, N 3.95; ber.: C 63.47, H 10.93, N $3.90 \%$.

\subsubsection{Darstellung von $\left[2,6-i \mathrm{Pr}_{2} \mathrm{C}_{6} \mathrm{H}_{3} \mathrm{~N}\left(\mathrm{SiMe}_{3}\right) \mathrm{AlOC}_{4} \mathrm{H}_{9}\right]_{2}$ (2)}

$1.70 \mathrm{~g}$ (4.73 mmol) der Verbindung 1 wurden in Diethylether $(30 \mathrm{~mL})$ gelöst und mit 3 Äq. Methyliodid (2.01 g, $14.19 \mathrm{mmol})$ versetzt und sechs Stunden lang bei Raumtemperatur gerührt. Nachdem man über Celite filtriert hat, wurden die leichtflüchtigen Bestandteile im Vakuum entfernt. Es blieb eine zähflüssige Masse übrig, von der ein ${ }^{1} \mathrm{H}-\mathrm{NMR}$-Spektrum angefertigt wurde.

${ }^{1} \mathrm{H}-\mathrm{NMR}\left(200.13 \mathrm{MHz}, \mathrm{C}_{6} \mathrm{D}_{6}\right.$ ): $\delta 7.20-7.05$ (m, $3 \mathrm{H}, \mathrm{Ar} H$ ), 3.86 (sept, $J=7.0 \mathrm{~Hz}, 2 \mathrm{H}$, $\left.\mathrm{CH}\left(\mathrm{CH}_{3}\right)_{2}\right), 3.41\left(\mathrm{q}, J=7.0 \mathrm{~Hz}, 4 \mathrm{H}, \mathrm{OCH}_{2}\right), 1.36\left(\mathrm{~d}, J=7.0 \mathrm{~Hz}, 6 \mathrm{H}, \mathrm{CH}\left(\mathrm{CH}_{3}\right)_{2}\right), 1.31$ $\left(\mathrm{d}, J=7.0 \mathrm{~Hz}, 6 \mathrm{H}, \mathrm{CH}\left(\mathrm{CH}_{3}\right)_{2}\right), 0.67$ (t, $\left.J=7.0 \mathrm{~Hz}, 6 \mathrm{H}, \mathrm{OCH}_{2}-\mathrm{CH}_{3}\right), 0.36\left(\mathrm{~s}, 9 \mathrm{H}, \mathrm{Si}\left(\mathrm{CH}_{3}\right)_{3}\right)$.

Der Rückstand wurde in THF $(15 \mathrm{~mL})$ aufgenommen und zum Kristallisieren bei $12{ }^{\circ} \mathrm{C}$ gelagert. Es bildeten sich nach einiger Zeit wenige klare Kristalle, von denen eine 
Röntgenstrukturanalyse angefertigt wurde. Nach Entfernen des Lösungsmittels und Anfertigen eines ${ }^{1} \mathrm{H}-\mathrm{NMR}-\mathrm{Spektrums}$ stellte sich heraus, dass es sich um ein Stoffgemisch handelte (sehr viele Signale, besonders im Bereich von $\delta 3-0$ ). Dieses konnte nicht weiter aufgetrennt werden.

\subsection{Umsetzung von Alkinyl-substituierten Titano- bzw. Zirconocen- Komplexen mit reaktiven Aluminiumverbindungen}

\subsubsection{Reaktion von reaktiven Aluminiumverbindungen mit $\mathrm{Cp}_{2} \mathrm{Ti}(\mathrm{C} \equiv \mathrm{CR})_{2} \quad(\mathrm{R}=\mathrm{Ph}$, $t$ Bu)}

Eine Lösung von $0.38 \mathrm{~g}(1.0 \mathrm{mmol}) \mathrm{Cp}_{2} \mathrm{Ti}(\mathrm{C} \equiv \mathrm{CPh})_{2}$ in $\mathrm{Et}_{2} \mathrm{O}$ wurde bei $-78{ }^{\circ} \mathrm{C}$ mit $1.0 \mathrm{mmol}$ $(0.072 \mathrm{~g}) \mathrm{AlMe}_{3}$ versetzt und 5 Tage bei RT lang gerührt. Die Lösung wurde braun. Nach Filtration und Waschen mit THF erhielt man einen grünen Filterrückstand, der so unlöslich war, dass es nicht möglich war, ein ${ }^{1} \mathrm{H}-\mathrm{NMR}-\mathrm{Spektrum}$ anzufertigen. Dieses Verhalten ist bereits von der Titanocen(III)-Verbindung $\left[\mathrm{Cp}_{2} \mathrm{Ti}(\mathrm{C} \equiv \mathrm{CPh})\right]_{2}$ bekannt. $^{[26,27,28]}$

Vom rötlichen Filtrat wurde das Lösungsmittel im Vakuum entfernt und ein ${ }^{1}$ H-NMRSpektrum angefertigt. Aufgrund vieler Signale unterschiedlicher Intensität, konnte man auf ein Stoffgemisch schließen, welches sich nicht weiter auftrennen ließ.

Anstelle von $\mathrm{AlMe}_{3}$ wurden auch 1.0 mmol a.) 1 (3.60 g) oder b.) 2,6$i \operatorname{Pr}_{2} \mathrm{C}_{6} \mathrm{H}_{3} \mathrm{~N}\left(\mathrm{SiMe}_{3}\right) \mathrm{AlMe}_{2}(0.31 \mathrm{~g})$ verwendet. Ebenfalls anstelle von $\mathrm{AlMe}_{3}$ wurden $1.5 \mathrm{~mL}$ einer ca. 0.7 M Lösung $\mathrm{AlH}_{3} \cdot \mathrm{NEt}_{3}$ in Toluol sowie $0.5 \mathrm{mmol}[\mathrm{RAlH}(\mu-\mathrm{H})]_{2}(0.37 \mathrm{~g})$ $\left(\mathrm{R}=\mathrm{N}\left(\mathrm{SiMe}_{3}\right) \mathrm{C}(\mathrm{Ph}) \mathrm{C}\left(\mathrm{SiMe}_{3}\right)_{2}\right)$ verwendet. In allen Fällen entstand das bekannte Produkt $\left[\mathrm{Cp}_{2} \operatorname{Ti}(\mathrm{C} \equiv \mathrm{CPh})\right]_{2}$.

Der Einsatz von $\mathrm{Cp}_{2} \mathrm{Ti}(\mathrm{C} \equiv \mathrm{C} t \mathrm{Bu})_{2}$ führte zu der besser löslichen, bekannten Titanocen(III)Verbindung $\left[\mathrm{Cp}_{2} \mathrm{Ti}(\mathrm{C} \equiv \mathrm{C} t \mathrm{Bu})\right]_{2}$, deren Entstehen anhand ihres ${ }^{1} \mathrm{H}-\mathrm{NMR}-$ Spektrums $\left({ }^{1} \mathrm{H}-\mathrm{NMR}\right.$ $\left.\left(200.13 \mathrm{MHz}, \mathrm{C}_{6} \mathrm{D}_{6}\right): \delta 5.52(\mathrm{~s}, 10 \mathrm{H}, \mathrm{Cp}), 1.21\left(\mathrm{~s}, 9 \mathrm{H}, \mathrm{C}_{4} H_{9}\right)\right)$ nachgewiesen werden konnte. ${ }^{[26,27,28]}$ 


\subsection{Darstellung einer neuen Klasse von Aluminium-Verbindungen des Typs $M\left[\operatorname{RAl}(C \equiv C R)_{3}\right]$ mit drei terminalen Ethinyl- Gruppen und deren Interaktion mit Alkalimetall- Kationen}

\subsubsection{Darstellung von $\left[2,6-i \mathrm{Pr}_{2} \mathrm{C}_{6} \mathrm{H}_{3} \mathrm{~N}\left(\mathrm{SiMe}_{3}\right) \mathrm{AlCl}_{2}\right]_{2}$ (3)}

[2,6-i $\left.\mathrm{Pr}_{2} \mathrm{C}_{6} \mathrm{H}_{3} \mathrm{~N}\left(\mathrm{SiMe}_{3}\right) \mathrm{AlMe}_{2}\right]_{2}(30.6 \mathrm{~g}, 50 \mathrm{mmol})$ in Toluol $(200 \mathrm{~mL})$ wurde bei $-78^{\circ} \mathrm{C}$ mit Trimethylzinnchlorid (39.9 g, 200 mmol) versetzt. Nach Entfernen des Kältebades wurde die Reaktionsmischung über Nacht bei RT gerührt. Die leichtflüchtigen Bestandteile wurden im Vakuum entfernt und das Rohprodukt mit wenig Pentan $(40 \mathrm{~mL})$ gewaschen. Nach Filtration wurde Verbindung 3 (24.2 g, 70\%) als farbloser Feststoff erhalten.

Smp.: $228^{\circ} \mathrm{C}$ (Zersetzung);

${ }^{1} \mathrm{H}-\mathrm{NMR}\left(200.13 \mathrm{MHz}, \mathrm{C}_{6} \mathrm{D}_{6}\right): \delta$ 7.1-6.8 (m, $3 \mathrm{H}, \mathrm{Ar} H$ ), 3.56 (sept, $J=7.5 \mathrm{~Hz}, 2 \mathrm{H}$, $\left.\mathrm{CH}\left(\mathrm{CH}_{3}\right)_{2}\right), 1.35$ (d, $\left.J=7.5 \mathrm{~Hz}, 6 \mathrm{H}, \mathrm{CH}\left(\mathrm{CH}_{3}\right)_{2}\right), 1.26\left(\mathrm{~d}, J=7.5 \mathrm{~Hz}, 6 \mathrm{H}, \mathrm{CH}\left(\mathrm{CH}_{3}\right)_{2}\right), 0.29$ (s, $\left.9 \mathrm{H}, \mathrm{Si}\left(\mathrm{CH}_{3}\right)_{3}\right)$;

$\mathrm{MS}(\mathrm{EI}): m / z(\%): 345$ (1/2(M), 50), 249 (1/2(M- $\left.\left.\mathrm{AlCl}_{2}\right), 100\right)$;

IR (Nujol): $\widetilde{v}=2120,2097,1597,1241,1181,925,759,723,691,576,539 \mathrm{~cm}^{-1}$;

EA für $\mathrm{C}_{30} \mathrm{H}_{52} \mathrm{Al}_{2} \mathrm{Cl}_{4} \mathrm{~N}_{2} \mathrm{Si}_{2}$ (692.70): gef.: $\mathrm{N}, 3.36$; ber.: $\mathrm{N}, 4.04 \%$.

\subsubsection{Darstellung von $\left[\mathrm{K} \cdot \mathrm{THF}\left(2,6-i \mathrm{Pr}_{2} \mathrm{C}_{6} \mathrm{H}_{3} \mathrm{~N}\left(\mathrm{SiMe}_{3}\right) \mathrm{Al}(\mathrm{C} \equiv \mathrm{CPh})_{3}\right)\right]_{2}(4)$}

$\mathrm{Zu}$ einer Lösung aus 3 in THF (200 mL) (1.73 g, $2.5 \mathrm{mmol})$ wurden 6 Äq KC $\equiv \mathrm{CPh}(1.73 \mathrm{~g}$, $2.5 \mathrm{mmol}$ ) gegeben und über Nacht bei $50{ }^{\circ} \mathrm{C}$ gerührt. Nach Abkühlen auf RT wurde das Lösungsmittel im Vakuum entfernt. Zu der Suspension wurde etwas Hexan (35 mL) gegeben, 5 Minuten gerührt und dann filtriert. Der Niederschlag wurde mit THF (250 mL) extrahiert. Es wurden 1.48 g (43 \%) des gewünschten Produkts erhalten. Geeignete Kristalle für die Röntgenstrukturanalyse konnten nach 18 Tagen durch langsames Eindiffundieren von Hexan in eine THF-Lösung von 4 erhalten werden.

Smp.: $>300{ }^{\circ} \mathrm{C}$;

${ }^{1} \mathrm{H}-\mathrm{NMR}\left(200.13 \mathrm{MHz}, \mathrm{THF}-\mathrm{D}_{8}\right): \delta 7.3-6.6$ (m, $\left.18 \mathrm{H}, \mathrm{ArH}\right), 4.12$ (sept, $J=7.0 \mathrm{~Hz}, 2 \mathrm{H}$, $\left.\mathrm{CH}\left(\mathrm{CH}_{3}\right)_{2}\right), 3.6\left(\mathrm{~m}, 4 \mathrm{H}, \mathrm{OCH}_{2} \mathrm{CH}_{2}\right), 1.8\left(\mathrm{~m}, 4 \mathrm{H}, \mathrm{OCH}_{2} \mathrm{CH}_{2}\right), 1.29(\mathrm{~d}, J=7.0 \mathrm{~Hz}, 6 \mathrm{H}$, $\left.\mathrm{CH}\left(\mathrm{CH}_{3}\right)_{2}\right), 1.18\left(\mathrm{~d}, J=7.0 \mathrm{~Hz}, 6 \mathrm{H}, \mathrm{CH}\left(\mathrm{CH}_{3}\right)_{2}\right), 0.18\left(\mathrm{~s}, 9 \mathrm{H}, \mathrm{Si}\left(\mathrm{CH}_{3}\right)_{3}\right)$; 
${ }^{13} \mathrm{C}-\mathrm{NMR}\left(125 \mathrm{MHz}, \mathrm{THF}-\mathrm{D}_{8}\right): \delta 149.38,147.71,132.17,128.51,127.73,127.01,122.85$, 121.58 (s, arom. C), 113.5 (br, Al-C), 106.11 (s, C-Ph), 68.24 (s, THF), 28.20 (s, THF), $28.11(\mathrm{~s}, \mathrm{CH}), 26.46\left(\mathrm{~s}, \mathrm{CH}_{3}\right), 3.37\left(\mathrm{SiMe}_{3}\right)$;

${ }^{29} \mathrm{Si}$ NMR (99 MHz, THF-D $): \delta-2.2$;

IR (Nujol): $\widetilde{v}=2118,2099,1654,1315,1263,1240,1183,1158,1053,927,798,759,691$, $576,539 \mathrm{~cm}^{-1}$;

MS(FAB): $m / z(\%): 578$ (1/2(M - 2 THF - K), 100), 1195 (( $M$ - 2 THF - K), 10);

EA für $\mathrm{C}_{86} \mathrm{H}_{98} \mathrm{Al}_{2} \mathrm{~K}_{2} \mathrm{~N}_{2} \mathrm{O}_{2} \mathrm{Si}_{2}$ (1380.07): gef.: C 74.08, H 7.33, N 1.87, ber.: C 74.85, H 7.16, $\mathrm{N} 2.03 \%$.

\subsubsection{Darstellung von $\left[\mathrm{Na} \cdot \mathrm{THF}\left(2,6-i \mathrm{Pr}_{2} \mathrm{C}_{6} \mathrm{H}_{3} \mathrm{~N}\left(\mathrm{SiMe}_{3}\right) \mathrm{Al}(\mathrm{C} \equiv \mathrm{CPh})_{3}\right)\right]_{2}(5)$}

Zu 3 (1.73 g, $2.5 \mathrm{mmol})$ in THF (200 mL) wurden $1.86 \mathrm{~g}$ (15 mmol, 6 Äq) NaC $\equiv \mathrm{CPh}$ gegeben und bei $50{ }^{\circ} \mathrm{C}$ über Nacht gerührt. Das Lösungsmittel wurde im Vakuum entfernt. Das schwer lösliche Rohprodukt wurde mit Toluol (35 mL) aufgeschlämmt und anschließend abfiltriert. Die erhaltene Mischung aus $\mathrm{NaCl}$ und 5 (3.30 g), die leicht gelb gefärbt war, war aufgrund der schlechten Löslichkeit in organischen Lösungsmitteln nicht weiter auftrennbar. Einkristalle für die Röntgenstrukturanalyse wurden erhalten, indem das Produkt in THF aufschlämmt wurde, die hellgelbe Lösung in ein Gläschen dekantiert wurde und anschließend das Gläschen in einen Kolben gestellt wurde, der Hexan enthielt. Durch das in die THFLösung eindiffundierende Hexan fielen nach 20 Tagen klare Kristalle aus.

Smp.: $>300^{\circ} \mathrm{C}$;

${ }^{1} \mathrm{H}-\mathrm{NMR}\left(200.13 \mathrm{MHz}, \mathrm{C}_{6} \mathrm{D}_{6}\right): \delta 7.50-6.70(\mathrm{~m}, 18 \mathrm{H}, \mathrm{Ar} H), 4.45$ (sept, $J=6.9,2 \mathrm{H}$, $\left.\mathrm{CH}\left(\mathrm{CH}_{3}\right)_{2}\right), 3.27\left(\mathrm{~m}, 4 \mathrm{H}, \mathrm{OCH}_{2} \mathrm{CH}_{2}\right), 1.57\left(\mathrm{~d}, J=6.9,6 \mathrm{H}, \mathrm{CH}\left(\mathrm{CH}_{3}\right)_{2}\right), 1.55(\mathrm{~d}, J=6.9,6 \mathrm{H}$, $\left.\mathrm{CH}\left(\mathrm{CH}_{3}\right)_{2}\right), 1.06\left(\mathrm{~m}, 4 \mathrm{H}, \mathrm{OCH}_{2} \mathrm{CH}_{2}\right), 0.67$ (s, $\left.9 \mathrm{H}, \mathrm{Si}\left(\mathrm{CH}_{3}\right)_{3}\right)$;

${ }^{13} \mathrm{C}-\mathrm{NMR}\left(125 \mathrm{MHz}, \mathrm{THF}-\mathrm{D}_{8}\right): \delta$ 149.69, 147.75, 132.22, 128.50, 128.34, 128.26, 122.76, 121.38 (s, arom. C), 112.5 (br, Al-C), 105.40 (s, C-Ph), 68.20 (s, THF), 28.08 (s, THF), 28.04 (s, CH), $26.36\left(\mathrm{~s}, \mathrm{CH}_{3}\right), 3.38\left(\mathrm{SiMe}_{3}\right)$;

${ }^{29} \mathrm{Si} \mathrm{NMR}\left(99 \mathrm{MHz}, \mathrm{C}_{6} \mathrm{D}_{6}\right): \delta 0.6$;

IR (Nujol): $\widetilde{v}=2120,2098,1597,1572,1240,1184,1156,1049,925,759,691,576$, $539 \mathrm{~cm}^{-1}$. 


\subsubsection{Darstellung von $\left[\mathrm{Li} \cdot \operatorname{Dioxan}\left(2,6-i \mathrm{Pr}_{2} \mathrm{C}_{6} \mathrm{H}_{3} \mathrm{~N}\left(\mathrm{SiMe}_{3}\right) \mathrm{Al}(\mathrm{C} \equiv \mathrm{CPh})_{3}\right)\right]_{2} \cdot 2$ Dioxan (6)}

$\mathrm{Zu}$ einer Lösung aus $\mathrm{HC} \equiv \mathrm{CPh}(3.06 \mathrm{~g}, 30 \mathrm{mmol})$ in Diethylether $(100 \mathrm{~mL})$ wurde bei $-78^{\circ} \mathrm{C}$ eine 1-M Lösung von $n$-BuLi in Hexan $(30 \mathrm{mmol})$ gegeben. Die Reaktionsmischung wurde bei RT über Nacht gerührt. Verbindung 3 (3.46 g, $5 \mathrm{mmol}$ ) wurde zu dieser Lösung bei $-78{ }^{\circ} \mathrm{C}$ gegeben. Anschließend wurde die Mischung langsam auf RT erwärmt und über Nacht gerührt. Nach Filtration wurde vom Filtrat das Lösungsmittel entfernt. Zu dem öligen Rückstand wurde dann Dioxan (50 mL) gegeben und die Mischung 4 Stunden lang gerührt. Nachdem das Dioxan im Vakuum entfernt worden ist, wurde das Rohprodukt mit $35 \mathrm{~mL}$ Hexan gewaschen und es wurden $4.31 \mathrm{~g}(60 \%)$ des weißen Feststoffs 6 erhalten.

Smp.: $>300^{\circ} \mathrm{C}$;

${ }^{1} \mathrm{H}-\mathrm{NMR}\left(200.13 \mathrm{MHz}, \mathrm{C}_{6} \mathrm{D}_{6}\right.$ ): $\delta 7.40-6.80$ (m, $18 \mathrm{H}, \mathrm{ArH}$ ), 4.40 (sept, $J=6.8 \mathrm{~Hz}, 2 \mathrm{H}$, $\left.\mathrm{CH}\left(\mathrm{CH}_{3}\right)_{2}\right), 3.38$ (s, $18 \mathrm{H}$, Dioxan), 1.61 (d, $\left.J=6.8 \mathrm{~Hz}, 6 \mathrm{H}, \mathrm{CH}\left(\mathrm{CH}_{3}\right)_{2}\right), 1.51$ (d, $J=6.8 \mathrm{~Hz}$, $\left.6 \mathrm{H}, \mathrm{CH}\left(\mathrm{CH}_{3}\right)_{2}\right), 0.66\left(\mathrm{~s}, 9 \mathrm{H}, \mathrm{Si}\left(\mathrm{CH}_{3}\right)_{3}\right)$;

${ }^{7} \mathrm{Li}-\mathrm{NMR}\left(97.2 \mathrm{MHz}, \mathrm{C}_{6} \mathrm{D}_{6}\right): \delta 0.2$;

${ }^{13} \mathrm{C}-\mathrm{NMR}\left(125 \mathrm{MHz}, \mathrm{C}_{6} \mathrm{D}_{6}\right.$ ): $\delta$ 147.41, 147.30, 131.78, 128.60, 128.05 (s, arom. C), 109.0 (br, Al-C), 108.82 (s, C-Ph), 67.17 (s, Dioxan), 28.11 (s, CH), 26.49 (s, $\left.\mathrm{CH}_{3}\right), 3.36\left(\mathrm{SiMe}_{3}\right)$;

${ }^{29} \mathrm{Si}-\mathrm{NMR}\left(99 \mathrm{MHz}, \mathrm{C}_{6} \mathrm{D}_{6}\right): \delta 0.2$;

IR (Nujol): $\widetilde{v}=2123,2111,1597,1572,1486,1272,1239,1181,1122,1109,1079,925$, $872,796,759,693,445 \mathrm{~cm}^{-1}$;

MS(FAB): $m / z$ (\%): 592 (1/2(M - 4 Dioxan), 100), 585 (1/2(M- 4 Dioxan - $\left.\left.\mathrm{Li}^{+}\right), 25\right)$;

EA für $\mathrm{C}_{94} \mathrm{H}_{114} \mathrm{Al}_{2} \mathrm{Li}_{2} \mathrm{~N}_{2} \mathrm{O}_{8} \mathrm{Si}_{2}$ (1523.89): gef.: C 73.86, H 7.85, N 1.88; ber.: C 74.09, H 7.54, $\mathrm{N} 1.84 \%$.

\subsection{Darstellung einer neuen Klasse von Verbindungen der 13. Gruppe des Periodensystems mit drei terminalen Trimethylsilylethinyl- Liganden des Typs $\left[\mathrm{RM}\left(\mathrm{C} \equiv \mathrm{CSiMe}_{3}\right)_{3}\right]^{-}$}

\subsection{1. $\left[\mathrm{Li} \cdot 2\right.$ Dioxan $\left.\left(2,6-i \mathrm{Pr}_{2} \mathrm{C}_{6} \mathrm{H}_{3} \mathrm{~N}\left(\mathrm{SiMe}_{3}\right) \mathrm{Al}\left(\mathrm{C} \equiv \mathrm{CSiMe}_{3}\right)_{3}\right)\right] \cdot 0.75$ Dioxan (7)}

$\mathrm{Zu}$ einer Lösung aus $\mathrm{HC} \equiv \mathrm{CSiMe}_{3}(1.50 \mathrm{~g}, 15.3 \mathrm{mmol})$ in $\mathrm{THF}(75 \mathrm{~mL})$ wurde bei $-78^{\circ} \mathrm{C}$ eine Lösung von $n$-BuLi in Hexan (15.3 mmol) gegeben. Die Reaktionsmischung wurde bei RT über Nacht gerührt. Verbindung 3 (1.76 g, $2.55 \mathrm{mmol})$ wurde dann zu dieser Lösung bei 
$-78{ }^{\circ} \mathrm{C}$ gegeben. Anschließend wurde das Gemisch langsam auf RT erwärmt, über Nacht gerührt und abschließend $3 \mathrm{~h}$ auf $55^{\circ} \mathrm{C}$ erwärmt. Nach Abkühlen auf RT wurde das Lösungsmittel im Vakuum entfernt und mit Hexan versetzt. Filtration und Entfernen des Hexans vom Filtrat im Vakuum lieferte einen öligen Rückstand, welcher mit Dioxan (15 mL) versetzt wurde. Durch Lagerung der Lösung über Nacht konnten für die Röntgenstrukturanalyse farblose Einkristalle erhalten werden. Ausbeute: $2.83 \mathrm{~g}, 74 \%$.

Smp.: $>300{ }^{\circ} \mathrm{C}$;

${ }^{1} \mathrm{H}-\mathrm{NMR}\left(200.13 \mathrm{MHz}, \mathrm{THF}-\mathrm{D}_{8}\right): \delta 6.90-6.60$ (m, $\left.3 \mathrm{H}, \mathrm{ArH}\right), 4.02$ (sept, $J=6.9 \mathrm{~Hz}, 2 \mathrm{H}$, $\left.\mathrm{CH}\left(\mathrm{CH}_{3}\right)_{2}\right), 3.36\left(\mathrm{~s}, 8 \mathrm{H}\right.$, Dioxan), $1.24\left(\mathrm{~d}, J=6.9 \mathrm{~Hz}, 6 \mathrm{H}, \mathrm{CH}\left(\mathrm{CH}_{3}\right)_{2}\right), 1.13(\mathrm{~d}, J=6.9 \mathrm{~Hz}$, $\left.6 \mathrm{H}, \mathrm{CH}\left(\mathrm{CH}_{3}\right)_{2}\right), 0.07$ (s, $\left.9 \mathrm{H}, \mathrm{Si}\left(\mathrm{CH}_{3}\right)_{3}\right),-0.04$ (s, $\left.27 \mathrm{H}, \mathrm{C}_{\text {Alkinyl- }} \mathrm{Si}\left(\mathrm{CH}_{3}\right)_{3}\right)$;

${ }^{7} \mathrm{Li}-\mathrm{NMR}\left(97.2 \mathrm{MHz}, \mathrm{THF}-\mathrm{D}_{8}\right): \delta-0.3$;

${ }^{13} \mathrm{C}-\mathrm{NMR}\left(125 \mathrm{MHz}, \mathrm{THF}-\mathrm{D}_{8}\right.$ ): $\delta 150.5,147.6,122.5,120.9$ (arom. C), 109.0 (br, Al-C), 95.3 (C-TMS), 67.9 (Dioxan), 27.7 (CH), $27.1\left(\mathrm{CH}_{3}\right), 3.4\left(\mathrm{NSiMe}_{3}\right), 1.0\left(\mathrm{CSiMe}_{3}\right)$;

${ }^{27}$ Al-NMR (65 MHZ, THF-D $): \delta 84.3$;

${ }^{29} \mathrm{Si}-\mathrm{NMR}\left(99 \mathrm{MHz}, \mathrm{THF}-\mathrm{D}_{8}\right): \delta$-3.6, -25.3;

IR (Nujol): $\tilde{v}=2089,2054,1316,1262,1250,1184,1106,1075,931,870,853,841,795$, $759,733,686,451 \mathrm{~cm}^{-1}$;

MS(FAB): M(Anion) (\%): 566 ((M(Anion) - Dioxan - Li), 100);

EA für $\mathrm{C}_{38} \mathrm{H}_{69} \mathrm{AlLiNO}_{4} \mathrm{Si}_{4}$ (mit 2 koordinierten Dioxan-Molekülen) (750.23): gef.: C 59.67, H 9.11, N 1.89; ber.: C 60.8, H 9.3, N $1.9 \%$.

Alternative Darstellung von 7: Zu $1.50 \mathrm{~g}(4.2 \mathrm{mmol}) 1$ in Toluol $(30 \mathrm{~mL})$ wurden $1.65 \mathrm{~g}$ (4 Äq) $\mathrm{HC} \equiv \mathrm{CSiMe}_{3}$ gegeben. Man ließ das Gemisch vier Tage lang bei RT rühren, entfernte das Lösungsmittel im Vakuum nahm den Rückstand in THF (20 mL) auf und filtrierte über Celite. Nachdem das Lösungsmittel des Filtrats im Vakuum entfernt worden ist, wurden $5 \mathrm{~mL}$ Dioxan hinzugegeben. Das Dioxan wird im Vakuum entfernt, der Rückstand mit Pentan gewaschen und man erhält $1.63 \mathrm{~g}(52 \%) 7$.

\subsubsection{Versuche zur Darstellung von $\mathrm{RAI}\left(\mathrm{C} \equiv \mathrm{CSiMe}_{3}\right)_{2} \quad\left(\mathrm{R}=2,6-i \mathrm{Pr}_{2} \mathrm{C}_{6} \mathrm{H}_{3} \mathrm{~N}\left(\mathrm{SiMe}_{3}\right)\right)$}

$0.69 \mathrm{~g}$ ( $1 \mathrm{mmol}$ ) der Verbindung 3 wurden mit $0.42 \mathrm{~g}$ (4 Äq) $\mathrm{LiC} \mathrm{CSSiMe}_{3}$ in $\mathrm{THF}(30 \mathrm{~mL})$ versetzt und über Nacht bei RT gerührt. Anschließend wurde das Gemisch über Celite filtriert und vom Filtrat das Lösungsmittel im Vakuum entfernt. Nach Zugabe von Dioxan $(5 \mathrm{~mL})$ 
wurde das Gemisch 6 Stunden lang bei RT stehen gelassen. Nach dem Entfernen des Lösungsmittels im Vakuum wurde ein ${ }^{1} \mathrm{H}-\mathrm{NMR}-$ Spektrum angefertigt.

Neben den Signalen von 7 waren viele weitere Signale zu sehen, die nicht zugeordnet werden konnten.

$0.82 \mathrm{~g}$ ( $1 \mathrm{mmol}) 7$ wurden in THF (30 mL) mit a.) $3 \ddot{A q}(0.43 \mathrm{~g})$ MeI oder b.) $3 \ddot{A q}(0.20 \mathrm{~g})$ TMSCl bei RT versetzt und 24 Stunden gerührt. Nach Entfernen der leichtflüchtigen Bestandteile im Vakuum wiesen die angefertigten ${ }^{1} \mathrm{H}-\mathrm{NMR}-$ Spektren der zurückbleibenden zähflüssigen Masse sehr viele Signale auf, die nicht zugeordnet werden konnten.

\subsubsection{Darstellung von $\left[\mathrm{Li} \cdot \mathrm{Et}_{2} \mathrm{O}\left(2,6-i \mathrm{Pr}_{2} \mathrm{C}_{6} \mathrm{H}_{3} \mathrm{~N}\left(\mathrm{SiMe}_{3}\right) \mathrm{GaCl}_{3}\right)\right]$}

$\mathrm{Zu} 12.77 \mathrm{~g}$ (50 mmol) 2,6-i $\mathrm{Pr}_{2} \mathrm{C}_{6} \mathrm{H}_{3} \mathrm{~N}\left(\mathrm{SiMe}_{3}\right) \mathrm{Li}$ in Diethylether (150 mL) wurde unter Rühren bei $-78{ }^{\circ} \mathrm{C}$ eine Lösung aus $8.80 \mathrm{~g}(50 \mathrm{mmol}) \mathrm{GaCl}_{3}$ in Diethylether gegeben. Nach Entfernen des Kältebads erwärmte sich das Gemisch langsam auf RT wurde weitere 4 Stunden gerührt. Anschließend entfernte man das Lösungsmittel im Vakuum und wusch den Rückstand mit Pentan. Das Produkt wurde mittels einer Soxhlet-Apparatur mit $\mathrm{CHCl}_{3}$ extrahiert. Man erhielt $6.49 \mathrm{~g}(26 \%)$ des hellgelben Produktes.

Smp.: $98^{\circ} \mathrm{C}$;

${ }^{1} \mathrm{H}-\mathrm{NMR}\left(200.13 \mathrm{MHz}, \mathrm{THF}-\mathrm{D}_{8}\right): \delta 7.20-6.70(\mathrm{~m}, 3 \mathrm{H}, \mathrm{Ar} H), 3.87$ (sept, $\left.2 \mathrm{H}, \mathrm{CH}\left(\mathrm{CH}_{3}\right)_{2}\right)$, $3.38\left(\mathrm{q}, J=6.8 \mathrm{~Hz}, 4 \mathrm{H}, \mathrm{OCH}_{2}\right), 1.14\left(\mathrm{~d}, J=6.8 \mathrm{~Hz}, 6 \mathrm{H}, \mathrm{CH}\left(\mathrm{CH}_{3}\right)_{2}\right), 1.13(\mathrm{~d}, J=6.8 \mathrm{~Hz}, 6$ $\left.\mathrm{H}, \mathrm{CH}\left(\mathrm{CH}_{3}\right)_{2}\right), 1.11\left(\mathrm{t}, J=6.8 \mathrm{~Hz}, 6 \mathrm{H}, \mathrm{OCH}_{2}-\mathrm{CH}_{3}\right), 0.07$ (s, $\left.9 \mathrm{H}, \mathrm{Si}\left(\mathrm{CH}_{3}\right)_{3}\right)$;

${ }^{7} \mathrm{Li}-\mathrm{NMR}\left(97.2 \mathrm{MHz}, \mathrm{THF}-\mathrm{D}_{8}\right): \delta-0.43$;

${ }^{13} \mathrm{C}-\mathrm{NMR}$ (125 MHz, THF-D 8 ): $\delta$ 148.3, 147.0, 124.2, 123.0 (arom. C), $66.3\left(\mathrm{OCH}_{2}\right), 27.8$ $(\mathrm{CH}), 26.0\left(\mathrm{CH}_{3}\right), 15.7\left(\mathrm{CH}_{2} \mathrm{CH}_{3}\right), 3.1\left(\mathrm{SiMe}_{3}\right)$;

MS(EI): $m / z(\%): 389$ ((M- LiCl - $\left.\left.\mathrm{Et}_{2} \mathrm{O}\right), 30\right), 354\left(\left(M-\mathrm{Cl}-\mathrm{LiCl}-\mathrm{Et}_{2} \mathrm{O}\right), 15\right), 248\left(\left(M-\mathrm{GaCl}_{2}\right.\right.$ - $\left.\left.\mathrm{LiCl}-\mathrm{Et}_{2} \mathrm{O}\right), 100\right)$;

IR (Nujol): $\widetilde{v}=1433,1336,1317,1249,1186,1105,1041,915,886,870,834,803,751 \mathrm{~cm}^{-1}$; EA für $\mathrm{C}_{19} \mathrm{H}_{36} \mathrm{Cl}_{3} \mathrm{GaLiNOSi}$ (505.61): gef.: C 45.48, H 7.32, N 2.70, ber.: C 45.14, H 7.18, N $2.77 \%$. 


\subsubsection{Darstellung von}

$\left[(\mathrm{Li})_{2} \cdot(\text { Dioxan })_{7}\right]_{0.5}\left[2,6-i \operatorname{Pr}_{2} \mathrm{C}_{6} \mathrm{H}_{3} \mathrm{~N}\left(\mathrm{SiMe}_{3}\right) \mathrm{Ga}\left(\mathrm{C} \equiv \mathrm{CSiMe}_{3}\right)_{3}\right] \cdot 1.5 \operatorname{Dioxan}(9)$

$0.51 \mathrm{~g}(1 \mathrm{mmol}) 8$ in $\operatorname{THF}(75 \mathrm{~mL})$ wurden bei $-78{ }^{\circ} \mathrm{C}$ mit $3 \ddot{\mathrm{Aq}}(0.31 \mathrm{~g} ; 3 \mathrm{mmol})$ $\mathrm{LiC} \equiv \mathrm{CSiMe}_{3}$ versetzt. Man rührte das Gemisch 24 Stunden lang bei RT, entfernte dann das Lösungsmittel im Vakuum, gab Toluol $(40 \mathrm{~mL})$ zu und filtrierte über Celite. Das Filtrat wurde mit Dioxan $(10 \mathrm{~mL})$ versetzt und 24 Stunden bei RT gelagert. Die Lösungsmittel wurden anschließend im Vakuum entfernt, der Rückstand mit $4 \mathrm{~mL}$ kaltem Pentan gewaschen und in $30 \mathrm{~mL}$ Toluol aufgenommen. Die Lösung wurde vier Tage lang bei $-32{ }^{\circ} \mathrm{C}$ gelagert bis das Produkt vollständig auskristallisiert war. Nach Entnahme eines Einkristalls wurde das Produkt abfiltriert und im Vakuum getrocknet. Es wurden $0.48 \mathrm{~g}$ (61\%) der farblosen Verbindung 9 erhalten (mit 2 koordinierten Dioxan-Molekülen).

Smp.: $>300{ }^{\circ} \mathrm{C}$;

${ }^{1} \mathrm{H}-\mathrm{NMR}\left(200 \mathrm{MHz}, \mathrm{THF}-\mathrm{D}_{8}\right): \delta 7.00-6.50(\mathrm{~m}, 3 \mathrm{H}, \mathrm{Ar} H), 4.05$ (sept, $J=6.7 \mathrm{~Hz}, 2 \mathrm{H}$, $\left.\mathrm{CH}\left(\mathrm{CH}_{3}\right)_{2}\right), 3.58\left(\mathrm{~s}, 8 \mathrm{H}\right.$, Dioxan), $1.21\left(\mathrm{~d}, J=6.7 \mathrm{~Hz}, 6 \mathrm{H}, \mathrm{CH}\left(\mathrm{CH}_{3}\right)_{2}\right), 1.12(\mathrm{~d}, J=6.7 \mathrm{~Hz}$, $\left.6 \mathrm{H}, \mathrm{CH}\left(\mathrm{CH}_{3}\right)_{2}\right), 0.07$ (s, $\left.9 \mathrm{H}, \mathrm{Si}\left(\mathrm{CH}_{3}\right)_{3}\right),-0.03$ (s, $\left.27 \mathrm{H}, \mathrm{C}_{\text {Alkinyl }}-\mathrm{Si}\left(\mathrm{CH}_{3}\right)_{3}\right)$;

${ }^{7} \mathrm{Li}-\mathrm{NMR}\left(97.2 \mathrm{MHz}, \mathrm{THF}-\mathrm{D}_{8}\right): \delta-0.5$;

${ }^{13} \mathrm{C}-\mathrm{NMR}\left(125 \mathrm{MHz}, \mathrm{THF}-\mathrm{D}_{8}\right): \delta 151.8,148.1,123.4,120.6,107.3$ (br), 95.2, 27.6, 26.9, 3.6, 1.0 ;

${ }^{29} \mathrm{Si}-\mathrm{NMR}\left(99 \mathrm{MHz}, \mathrm{THF}-\mathrm{D}_{8}\right): \delta-4.2,-25.2$;

IR (Nujol): $\widetilde{v}=2074,2062,1315,1261,1247,1191,1105,1051,1041,936,848,759,687$ $\mathrm{cm}^{-1}$;

MS(EI): $m / z$ (\%): 616 ((M - 2 Dioxan- H), 15), 511 ((M- 2 Dioxan - Li - C $\left.\left.\equiv \mathrm{CSSiMe}_{3}\right), 100\right)$;

EA für $\mathrm{C}_{38} \mathrm{H}_{69} \mathrm{GaLiNO}_{4} \mathrm{Si}_{4}$ (mit 2 koordinierten Dioxan-Molekülen) (792.98): gef.: C 56.82, H 8.39, N 1.60; ber.: C 57.2, H 8.8, N $1.6 \%$;

\subsubsection{Darstellung von}

$\left[(\mathrm{Li})_{2} \cdot(\text { Dioxan })_{7}\right]_{0.5}\left[2,6-i \operatorname{Pr}_{2} \mathrm{C}_{6} \mathrm{H}_{3} \mathrm{~N}\left(\mathrm{SiMe}_{3}\right) \operatorname{In}\left(\mathrm{C} \equiv \mathrm{CSiMe}_{3}\right)_{3}\right] \cdot 1.5$ Dioxan (10)

$0.69 \mathrm{~g}(1 \mathrm{mmol})\left[\mathrm{Li} \cdot 3 \mathrm{THF}\left(2,6-i \mathrm{Pr}_{2} \mathrm{C}_{6} \mathrm{H}_{3} \mathrm{~N}\left(\mathrm{SiMe}_{3}\right) \mathrm{InCl}_{3}\right)\right]$ in Toluol $(60 \mathrm{~mL})$ wurden bei $-78{ }^{\circ} \mathrm{C}$ mit $3 \ddot{\mathrm{Aq}} \mathrm{LiC} \equiv \mathrm{CSiMe}_{3}(0.31 \mathrm{~g})$ versetzt, das Gemisch dann 3 Tage lang bei RT gerührt und anschließend über Celite filtriert. Nachdem die Mischung im Vakuum bis auf ca. $10 \mathrm{~mL}$ eingeengt und dann mit $5 \mathrm{~mL}$ Dioxan versetzt wurde, lagerte man das Gemisch 
5 Tage lang. Nach Entnahme eines farblosen Einkristalls für eine Röntgenstrukturanalyse wurde das Produkt abfiltriert und mit $3 \mathrm{~mL}$ Pentan gewaschen. Nach Trocknen im Vakuum erhielt man 0.82 g $(74 \%)$ des gewünschten Produkts 10 (mit zwei koordinierenden DioxanMolekülen).

Zers.: $160^{\circ} \mathrm{C}$;

${ }^{1} \mathrm{H}-\mathrm{NMR}\left(200.13 \mathrm{MHz}, \mathrm{THF}-\mathrm{D}_{8}\right): \delta$ 7.0-6.5 (m, $\left.3 \mathrm{H}, \mathrm{Ar} H\right), 4.02$ (sept, $J=7.0 \mathrm{~Hz}, 2 \mathrm{H}$, $\left.\mathrm{CH}\left(\mathrm{CH}_{3}\right)_{2}\right), 3.56(\mathrm{~s}, 8 \mathrm{H}), 1.18(\mathrm{~d}, J=7.0 \mathrm{~Hz}, 6 \mathrm{H}), 1.12(\mathrm{~d}, J=7.0 \mathrm{~Hz}, 6 \mathrm{H}), 0.06(\mathrm{~s}, 9 \mathrm{H})$, $-0.02(\mathrm{~s}, 27 \mathrm{H})$;

${ }^{7} \mathrm{Li}-\mathrm{NMR}\left(97.2 \mathrm{MHz}, \mathrm{THF}-\mathrm{D}_{8}\right): \delta-0.2$;

${ }^{13} \mathrm{C}-\mathrm{NMR}$ (125 MHz, THF-D 8 ): $\delta 152.3,147.3,122.4,120.1,114.1$ (br), 95.2, 27.5, 26.5, 3.6, 1.1 ;

${ }^{29} \mathrm{Si}-\mathrm{NMR}\left(99 \mathrm{MHz}, \mathrm{THF}-\mathrm{D}_{8}\right): \delta-4.5,-25.2$;

IR (Nujol): $\widetilde{v}=2063,2051,1250,1123,1080,1032,941,876,845,760,722,676,606 \mathrm{~cm}^{-1}$; EA für $\mathrm{C}_{38} \mathrm{H}_{69} \mathrm{InLiNO}_{4} \mathrm{Si}_{4}$ (mit 2 koordinierenden Dioxan-Molekülen) (792.98): gef.: C 53.85, H 7.71, N 1.14; ber.: C 54.46, H 8.30, N $1.67 \%$.

\subsubsection{Darstellung der Verbindung $\left[\mathrm{Li} \cdot 2\right.$ THF $\left.\left(2,6-i \mathrm{Pr}_{2} \mathrm{C}_{6} \mathrm{H}_{3} \mathrm{~N}\left(\mathrm{SiMe}_{3}\right) \operatorname{In}\left(\mathrm{C} \equiv \mathrm{CSiMe}_{3}\right)_{3}\right)\right]$} (11)

$0.69 \mathrm{~g}(1.0 \mathrm{mmol})\left[\mathrm{Li} \cdot 3 \mathrm{THF}\left(2,6-i \mathrm{Pr}_{2} \mathrm{C}_{6} \mathrm{H}_{3} \mathrm{~N}\left(\mathrm{SiMe}_{3}\right) \mathrm{InCl}_{3}\right)\right]$ in Toluol $(40 \mathrm{~mL})$ wurden bei $-78{ }^{\circ} \mathrm{C}$ mit $3 \ddot{\mathrm{Aq} \mathrm{LiC}} \equiv \mathrm{CSiMe}_{3}(0.31 \mathrm{~g})$ in $20 \mathrm{~mL}$ THF versetzt, das Gemisch dann 3 Tage lang bei RT gerührt und anschließend über Celite filtriert. Die Mischung wurde im Vakuum bis auf ca. $10 \mathrm{~mL}$ eingeengt und 6 Tage bei $-30{ }^{\circ} \mathrm{C}$ gelagert. Nach einigen Stunden bildeten sich farblose Einkristalle, die durch Röntgenstrukturanalyse charakterisiert werden konnten. Anschließend wurde das auskristallisierte Produkt bei $-30{ }^{\circ} \mathrm{C}$ abfiltriert und im Vakuum getrocknet. Es wurden 0.70 g $(81 \%)$ des gewünschten Produkts 11 erhalten.

Zers.: $142{ }^{\circ} \mathrm{C}$;

${ }^{1} \mathrm{H}-\mathrm{NMR}\left(200.13 \mathrm{MHz}, \mathrm{THF}-\mathrm{D}_{8}\right): \delta 7.10-6.60$ (m, $3 \mathrm{H}, \mathrm{Ar} H$ ), 3.86 (sept, $J=7.0 \mathrm{~Hz}, 2 \mathrm{H}$, $\left.\mathrm{CH}\left(\mathrm{CH}_{3}\right)_{2}\right), 3.64\left(\mathrm{~m}, 8 \mathrm{H}, \mathrm{OCH}_{2}-\mathrm{CH}_{2}\right), 1.80\left(\mathrm{~m}, 8 \mathrm{H}, \mathrm{OCH}_{2}-\mathrm{CH}_{2}\right), 1.14(\mathrm{~d}, J=7.0 \mathrm{~Hz}, 6 \mathrm{H}$, $\left.\mathrm{CH}\left(\mathrm{CH}_{3}\right)_{2}\right), 1.10\left(\mathrm{~d}, J=7.0 \mathrm{~Hz}, 6 \mathrm{H}, \mathrm{CH}\left(\mathrm{CH}_{3}\right)_{2}\right), 0.10\left(\mathrm{~s}, 27 \mathrm{H}, \mathrm{C}_{\text {Alkinyl }}-\mathrm{Si}\left(\mathrm{CH}_{3}\right)_{3}\right), 0.01$ (s, $\left.9 \mathrm{H}, \mathrm{Si}\left(\mathrm{CH}_{3}\right)_{3}\right)$; 
${ }^{7} \mathrm{Li}-\mathrm{NMR}\left(97.2 \mathrm{MHz}, \mathrm{THF}-\mathrm{D}_{8}\right): \delta-0.3$;

${ }^{13}$ C-NMR (125 MHz, THF-D 8 ): $\delta 152.3,145.6,122.4,120.1,111.7$ (br), 95.2, 27.5, 26.5, 3.6, 1.1

${ }^{29} \mathrm{Si}-\mathrm{NMR}\left(99 \mathrm{MHz}, \mathrm{THF}-\mathrm{D}_{8}\right): \delta-6.4,-27.0$;

IR (Nujol): $\tilde{v}=2064,2038,1996,1619,1586,1315,1250,1179,1123,1081,1046,937$, $876,846,760,676,606 \mathrm{~cm}^{-1}$;

EA für $\mathrm{C}_{38} \mathrm{H}_{69} \mathrm{InLiNO}_{2} \mathrm{Si}_{4}$ (806.07): gef.: C 55.87, H 7.81 N 1.38; ber.: C 56.62, H 8.63, N $1.73 \%$.

\subsection{Darstellung von Metall-Halogen-Diethinyl-Verbindungen der 13 .} Gruppe des Periodensystems des Typs $\mathrm{X}-\mathrm{M}(\mathrm{C} \equiv \mathrm{C} t \mathrm{Bu})_{2} \cdot \mathrm{n} \mathrm{S}(\mathrm{M}=\mathrm{Al}$, Ga, In; X = Cl, Br; S = THF; $n=1,2$ )

\subsubsection{Darstellung von $\mathrm{Br}-\mathrm{Al}(\mathrm{C} \equiv \mathrm{C} t \mathrm{Bu})_{2} \cdot 2$ THF (12)}

$0.53 \mathrm{~g}$ (2.00 mmol) $\mathrm{AlBr}_{3}$ wurden in $2 \mathrm{~mL}$ Hexan suspendiert und bei $-78{ }^{\circ} \mathrm{C}$ mit $\mathrm{THF}$ $(60 \mathrm{~mL})$ versetzt. Diese Mischung wurde bei $-78{ }^{\circ} \mathrm{C}$ langsam mit 2 Äq (0.35 g, $\left.4.0 \mathrm{mmol}\right)$ $\mathrm{LiC} \equiv \mathrm{C} t \mathrm{Bu}$ versetzt und 2 Tage lang bei RT gerührt. Das Lösungsmittel wurde im Vakuum entfernt und mit Hexan $(150 \mathrm{~mL})$ versetzt. Nach Filtration über Celite wurde das Hexan des Filtrats im Vakuum entfernt. Es wurden $0.55 \mathrm{~g}$ (67\%) eines farblosen Feststoffs erhalten. Für die Röntgenstrukturanalyse geeignete Kristalle konnten durch Lagerung in Hexan bei $12{ }^{\circ} \mathrm{C}$ nach 5 Tagen erhalten werden.

Zers.: $145^{\circ} \mathrm{C}$;

${ }^{1} \mathrm{H}-\mathrm{NMR}\left(200.13 \mathrm{MHz}, \mathrm{C}_{6} \mathrm{D}_{6}\right): \delta 3.78\left(\mathrm{~m}, 8 \mathrm{H}, \mathrm{OCH}_{2}-\mathrm{CH}_{2}\right), 1.25\left(\mathrm{~m}, 8 \mathrm{H}, \mathrm{OCH}_{2}-\mathrm{CH}_{2}\right), 1.22$ $(\mathrm{s}, 18 \mathrm{H}, t \mathrm{Bu})$;

${ }^{13} \mathrm{C}-\mathrm{NMR}\left(125 \mathrm{MHz}, \mathrm{C}_{6} \mathrm{D}_{6}\right): \delta 92.6(\underline{\mathrm{C}}-\mathrm{tBu}), 67.9$ (br, Al-C), 67.2 (THF), 30.9 (Me), 27.3 ( $\left.\underline{\mathrm{C}}-\mathrm{Me}_{3}\right), 25.8$ (THF);

IR (Nujol): $\widetilde{v}=2165,2129,1577,1260,1251,1204,1091,1023,923,861,803,757,632$, $551,484 \mathrm{~cm}^{-1}$;

EA für $\mathrm{C}_{20} \mathrm{H}_{34} \mathrm{AlBrO}_{2}$ (413.37): gef.: C 57.30, H 8.07; ber.: C 58.11, H 8.29 \%. 


\subsubsection{Darstellung von $\mathrm{Cl}-\mathrm{Ga}(\mathrm{C} \equiv \mathrm{CtBu})_{2} \cdot \mathrm{THF}(13)$}

$0.30 \mathrm{~g}$ (1.70 mmol) $\mathrm{GaCl}_{3}$ wurden in $2 \mathrm{~mL}$ Hexan suspendiert und bei $-78{ }^{\circ} \mathrm{C}$ mit $\mathrm{THF}$ $(60 \mathrm{~mL})$ versetzt. Diese Mischung wurde bei $-78{ }^{\circ} \mathrm{C}$ langsam mit $2 \ddot{\mathrm{Aq}}(0.30 \mathrm{~g}, 3.41 \mathrm{mmol})$ $\mathrm{LiC} \equiv \mathrm{C} t \mathrm{Bu}$ versetzt und $2 \mathrm{~d}$ lang bei RT gerührt. Das Lösungsmittel wurde im Vakuum entfernt und mit Hexan $(150 \mathrm{~mL})$ versetzt. Nach Filtration wurde das Hexan des Filtrats im Vakuum entfernt. Man erhielt 0.38 g (66 \%) eines weißen Feststoffs. Für die Röntgenstrukturanalyse geeignete Kristalle konnten nach einigen Tagen durch Lagerung in Hexan bei $12{ }^{\circ} \mathrm{C}$ erhalten werden.

Zers.: $110^{\circ} \mathrm{C}$;

${ }^{1} \mathrm{H}-\mathrm{NMR}\left(200.13 \mathrm{MHz}, \mathrm{C}_{6} \mathrm{D}_{6}\right): \delta 3.81\left(\mathrm{~m}, 4 \mathrm{H}, \mathrm{OCH}_{2}-\mathrm{CH}_{2}\right), 1.17(\mathrm{~s}, 18 \mathrm{H}, t \mathrm{Bu}), 1.08(\mathrm{~m}, 4 \mathrm{H}$, $\left.\mathrm{OCH}_{2}-\mathrm{CH}_{2}\right)$;

${ }^{13} \mathrm{C}-\mathrm{NMR}\left(125 \mathrm{MHz}, \mathrm{C}_{6} \mathrm{D}_{6}\right): \delta 116.4$ (C- $\left.t \mathrm{Bu}\right), 83.7$ (br, Ga-C), 70.2 (THF), $31.2(\mathrm{Me}), 28.3$ $\left(\underline{\mathrm{C}}-\mathrm{Me}_{3}\right), 25.0$ (THF);

IR (Nujol): $\widetilde{v}=2177,2144,1378,1366,1260,1253,1204,1096,1017,919,861,802,749$, $723,630,592,488 \mathrm{~cm}^{-1}$;

EA für $\mathrm{C}_{16} \mathrm{H}_{26} \mathrm{ClGaO}$ (339.56): gef.: C 56.29, H 7.73; ber.: C 56.60, H $7.72 \%$.

\subsubsection{Darstellung von $\mathrm{Cl}-\mathrm{In}(\mathrm{C} \equiv \mathrm{C} t \mathrm{Bu})_{2} \cdot 2$ THF (14)}

$0.45 \mathrm{~g}(2.04 \mathrm{mmol}) \mathrm{InCl}_{3}$ wurden mit $2 \mathrm{~mL}$ Hexan überschichtet und unter Rühren bei $-78^{\circ} \mathrm{C}$ mit THF (60 mL) versetzt. Diese Mischung wurde bei $-78^{\circ} \mathrm{C}$ langsam mit 2 Äq $(0.36 \mathrm{~g}, 4.09$ mmol) $\mathrm{LiC} \equiv \mathrm{C} t \mathrm{Bu}$ versetzt und $2 \mathrm{~d}$ lang bei RT gerührt. Das Lösungsmittel wurde im Vakuum entfernt und und das Produkt mit Hexan $(150 \mathrm{~mL})$ extrahiert. Es wurden $0.35 \mathrm{~g}(38 \%)$ des Produkts als farbloser Feststoffs erhalten. Für die Röntgenstrukturanalyse geeignete Kristalle konnten durch Lagerung in Hexan bei $12{ }^{\circ} \mathrm{C}$ nach 4 Tagen erhalten werden.

Zers.: $112{ }^{\circ} \mathrm{C}$;

${ }^{1} \mathrm{H}-\mathrm{NMR}\left(200.13 \mathrm{MHz}, \mathrm{C}_{6} \mathrm{D}_{6}\right): \delta 3.84\left(\mathrm{~m}, 8 \mathrm{H}, \mathrm{OCH}_{2}-\mathrm{CH}_{2}\right), 1.30\left(\mathrm{~m}, 8 \mathrm{H}, \mathrm{OCH}_{2}-\mathrm{CH}_{2}\right), 1.24$ $(\mathrm{s}, 18 \mathrm{H}, t \mathrm{Bu})$;

${ }^{13} \mathrm{C}-\mathrm{NMR}\left(125 \mathrm{MHz}, \mathrm{C}_{6} \mathrm{D}_{6}\right): \delta 118.2(\underline{\mathrm{C}}-\mathrm{tBu}), 88.3(\mathrm{br}, \mathrm{In}-\mathrm{C}), 68.5(\mathrm{THF}), 31.6(\mathrm{Me}), 28.5$ ( $\left.\underline{\mathrm{C}}-\mathrm{Me}_{3}\right), 25.3$ (THF); 
IR (Nujol): $\widetilde{v}=2133,1363,1251,1204,1095,1037,916,874,804,739,670,630,547,462$ $\mathrm{cm}^{-1}$;

EA für $\mathrm{C}_{20} \mathrm{H}_{34} \mathrm{ClInO}_{2}$ (456.76): gef.: C 52.19, H 7.64; ber.: C 52.59, H 7.50 \%.

\subsubsection{Reaktionsverhalten der Verbindungen 12,13 und 14}

$\mathrm{Zu} 0.25 \mathrm{~g}$ (1 mmol) 2,6-i $\mathrm{Pr}_{2} \mathrm{C}_{6} \mathrm{H}_{3} \mathrm{~N}\left(\mathrm{SiMe}_{3}\right) \mathrm{Li}$ in THF wurde bei RT ein Äquivalent a.) 12 b.) 13 oder c.) 14 gegeben und die Mischung 24 Stunden lang rühren gelassen. Nach mehreren Stunden fiel ein weißer Niederschlag aus, welcher durch Filtration über Celite entfernt wurde. Vom Filtrat wurde das Lösungsmittel im Vakuum entfernt.

Das ${ }^{1} \mathrm{H}-\mathrm{NMR}-$ Spektrum zeigt, dass es sich bei dem Einsatz der Ausgangsverbindung 12 und 14 um ein Produktgemisch handelt, welches nicht weiter aufgetrennt werden konnte. Setzte man 13 als Ausgangsverbindung ein, bildeten sich in einer gesättigten THF-Lösung neben einem weißen Feststoff auch farblose Kristalle, die mittels Röntgenstrukturanalyse als $\mathbf{1 5}$ identifiziert werden konnten. Für weitere spektroskopische Untersuchungen reichte die Menge an Kristallen aus der Mischung leider nicht mehr aus.

\subsection{Darstellung von Metall-Triethinyl-Verbindungen der 13. Gruppe des Periodensystems des Typs $\mathrm{M}(\mathrm{C} \equiv \mathrm{C} t \mathrm{Bu})_{3} \cdot \mathrm{S}(\mathrm{M}=\mathrm{Al}$, Ga, In; $\mathrm{S}=$ Dioxan, $\mathbf{N E t}_{3}$ )}

\subsubsection{Darstellung von $\mathrm{Al}(\mathrm{C} \equiv \mathrm{C} t \mathrm{Bu})_{3} \cdot \operatorname{Dioxan}(16)$}

$0.20 \mathrm{~g}$ (1.53 mmol) $\mathrm{AlCl}_{3}$ wurden mit $2 \mathrm{~mL}$ Hexan überschichtet und bei $-78^{\circ} \mathrm{C}$ mit Dioxan (70 mL) versetzt. Diese Mischung wurde mit $3 \ddot{\mathrm{A} q}(0.40 \mathrm{~g}, 4.6 \mathrm{mmol}) \mathrm{LiC} \equiv \mathrm{C} t \mathrm{Bu}$ versetzt und über Nacht bei RT gerührt. Nach Filtration über Celite wurde das Lösungsmittel des Filtrats im Vakuum entfernt und das Rohprodukt mit $5 \mathrm{~mL}$ Hexan gewaschen. Man erhielt $0.451 \mathrm{~g}$ (82\%) eines farblosen Feststoffs.

Zers.: $270{ }^{\circ} \mathrm{C}$;

${ }^{1} \mathrm{H}-\mathrm{NMR}\left(200.13 \mathrm{MHz}, \mathrm{THF}-\mathrm{D}_{8}\right): \delta 3.56$ (s, $8 \mathrm{H}$, Dioxan), 1.14 (s, $\left.27 \mathrm{H}, t \mathrm{Bu}\right)$;

${ }^{13} \mathrm{C}-\mathrm{NMR}\left(125 \mathrm{MHz}, \mathrm{THF}-\mathrm{D}_{8}\right): \delta 112.6$ (ㅌ-tBu), 94.1(br, Al-C), 67.8 (Dioxan), 32.2 (Me), $28.7\left(\underline{\mathrm{C}}-\mathrm{Me}_{3}\right)$;

IR (Nujol): $\widetilde{v}=2168,2125,1378,1362,1307,1260,1250,1204,1108,1069,919,892,860$, $751,606,537 \mathrm{~cm}^{-1}$; 
EA für $\mathrm{C}_{22} \mathrm{H}_{35} \mathrm{AlO}_{2}$ (358.50): gef.: C 72.84, H 9.66; ber.: C 73.71, H 9.84 \%.

\subsubsection{Darstellung von $\mathrm{Ga}(\mathrm{C} \equiv \mathrm{C} t \mathrm{Bu})_{3} \cdot \operatorname{Dioxan}(17)$}

$0.29 \mathrm{~g}$ (1.64 mmol) $\mathrm{GaCl}_{3}$ wurden mit $2 \mathrm{~mL}$ Hexan überschichtet und bei $-78^{\circ} \mathrm{C}$ mit Dioxan (70 mL) versetzt. Diese Mischung wurde mit 3 Äq $(0.44 \mathrm{~g}, 4.9 \mathrm{mmol}) \mathrm{LiC} \equiv \mathrm{C} t \mathrm{Bu}$ versetzt und über Nacht bei RT gerührt. Nach Filtration wurde das Lösungsmittel des Filtrats im Vakuum entfernt und das Rohprodukt mit Hexan $(5 \mathrm{~mL})$ gewaschen. Es wurden $0.50 \mathrm{~g}$ (67\%) eines farblosen Feststoffs erhalten. Für die Röntgenstrukturanalyse geeignete Kristalle konnten durch Lagerung in THF bei $-30{ }^{\circ} \mathrm{C}$ nach 10 Tagen erhalten werden.

Zers.: $330{ }^{\circ} \mathrm{C}$;

${ }^{1} \mathrm{H}-\mathrm{NMR}\left(200 \mathrm{MHz}, \mathrm{C}_{6} \mathrm{D}_{6}\right): \delta 3.54$ (s, $8 \mathrm{H}$, Dioxan), $1.15(\mathrm{~s}, 27 \mathrm{H}, t \mathrm{Bu})$;

${ }^{13} \mathrm{C}-\mathrm{NMR}\left(125 \mathrm{MHz}, \mathrm{C}_{6} \mathrm{D}_{6}\right.$ ): $\delta 116.5$ (- $\left.t \mathrm{Bu}\right), 86.3$ (br, Ga-C), 67.3 (Dioxan), 31.3 (Me), 28.3 $\left(\underline{\mathrm{C}}-\mathrm{Me}_{3}\right)$;

IR (Nujol): $\widetilde{v}=2171,2138,1363,1304,1251,1204,1112,1094,1077,1043,1030,889$, $860,803,743,609,551,478 \mathrm{~cm}^{-1}$;

MS(EI): $m / z$ (\%): 312 (M - Dioxan, 10), 297 (M - Dioxan - Me, 25), 231 (M - Dioxan $\mathrm{C} \equiv \mathrm{C} t \mathrm{Bu}, 100)$;

EA für $\mathrm{C}_{22} \mathrm{H}_{35} \mathrm{GaO}_{2}$ (401.24): gef.: C 65.46, H 9.06; ber.: C 65.86, H 8.79 \%.

\subsubsection{Darstellung von $\operatorname{In}(\mathrm{C} \equiv \mathrm{C} t \mathrm{Bu})_{3} \cdot \mathrm{NEt}_{3}(18)$}

$0.22 \mathrm{~g}(1.0 \mathrm{mmol}) \mathrm{InCl}_{3}$ wurden in $2 \mathrm{~mL}$ Hexan suspendiert und bei $-78{ }^{\circ} \mathrm{C}$ mit Triethylamin $(25 \mathrm{~mL})$ versetzt. Diese Mischung wurde mit $3 \ddot{\mathrm{Äq}}(0.26 \mathrm{~g}, 3.0 \mathrm{mmol}) \mathrm{LiC} \equiv \mathrm{C} t \mathrm{Bu}$ versetzt und über Nacht bei RT gerührt. Zu dem Gemisch wurden $50 \mathrm{~mL}$ THF gegeben und 24 Stunden lang bei RT gerührt. Die Lösungsmittel wurden im Vakuum entfernt und das Rohprodukt mit $70 \mathrm{~mL}$ Hexan versetzt. Nach Filtration wurde das Hexan im Vakuum entfernt und es konnten $0.38 \mathrm{~g}(83 \%)$ eines farblosen Feststoffs 18 erhalten werden. Für die Röntgenstrukturanalyse geeignete Einkristalle konnten durch Lagerung in Hexan bei $12^{\circ} \mathrm{C}$ nach 3 Tagen erhalten werden. Die Einkristalle enthalten noch ein halbes Hexanmolekül pro Elementarzelle, während das im Vakuum getrocknete Produkt kein Hexan mehr enthält. 
Zers.: $220{ }^{\circ} \mathrm{C}$;

${ }^{1} \mathrm{H}-\mathrm{NMR}\left(200.13 \mathrm{MHz}, \mathrm{C}_{6} \mathrm{D}_{6}\right): \delta 2.66$ (q, $\left.J=7.2 \mathrm{~Hz}, 6 \mathrm{H}, \mathrm{CH}_{2} \mathrm{CH}_{3}\right), 1.19(\mathrm{~s}, 27 \mathrm{H}, t \mathrm{Bu}), 1.08$ ( $\left.\mathrm{t}, J=7.2 \mathrm{~Hz}, 9 \mathrm{H}, \mathrm{CH}_{2} \mathrm{CH}_{3}\right)$;

${ }^{13} \mathrm{C}-\mathrm{NMR}\left(125 \mathrm{MHz}, \mathrm{C}_{6} \mathrm{D}_{6}\right): \delta 119.2(\underline{\mathrm{C}}-\mathrm{t} \mathrm{Bu}), 90.3(\mathrm{br}, \mathrm{In}-\mathrm{C}), 48.2\left(\mathrm{~N}-\underline{\mathrm{CH}}_{2}\right), 31.6(\mathrm{Me}), 28.5$ $\left(\underline{\mathrm{C}}-\mathrm{Me}_{3}\right), 10.7\left(\mathrm{CH}_{2}-\underline{\mathrm{CH}_{3}}\right)$;

IR (Nujol): $\widetilde{v}=2160,2124,1400,1362,1328,1261,1249,1204,1171,1101,1053,1026$, $908,807,748,735,547,453 \mathrm{~cm}^{-1}$;

EA für $\mathrm{C}_{24} \mathrm{H}_{42} \mathrm{InN}$ (459.42): gef.: C 61.93, H 8.75, N 2.31; ber.: C 62.74, H 9.22, N $3.05 \%$.

\subsection{Darstellung von Metall-Tetraethinyl- Verbindungen des Typs $\operatorname{Li}\left(\mathrm{Al}(\mathrm{C} \equiv t \mathrm{Bu})_{4}\right) \cdot \mathbf{n} \mathrm{THF}$}

\subsubsection{Darstellung von $\left[\mathrm{Li} \cdot \mathrm{THF}\left(\mathrm{Al}(\mathrm{C} \equiv \mathrm{Ct} \mathrm{Bu})_{4}\right)\right](19)$}

$0.24 \mathrm{~g}$ (6.32 mmol) Lithiumaluminiumhydrid wurden bei $-78^{\circ} \mathrm{C}$ in THF $(40 \mathrm{~mL})$ gelöst und mit 4.5 Äq (28.46 mmol; $2.34 \mathrm{~g} ; 3.5 \mathrm{~mL}$ ) $\mathrm{HC} \equiv \mathrm{C} t \mathrm{Bu}$ versetzt. Das Gemisch wurde 7 Tage lang bei RT gerührt und anschließend über Celite filtriert. Nach Entfernen der leichtflüchtigen Bestandteile im Vakuum wurden $0.89 \mathrm{~g}$ (33 \%) des gewünschten farblosen Produkts erhalten.

Zers.: $153{ }^{\circ} \mathrm{C}$;

${ }^{1} \mathrm{H}-\mathrm{NMR}\left(200.13 \mathrm{MHz}, \mathrm{THF}-\mathrm{D}_{8}\right): \delta 3.62\left(\mathrm{~m}, 4 \mathrm{H}, \mathrm{OCH}_{2}-\mathrm{CH}_{2}\right), 1.77\left(\mathrm{~m}, 4 \mathrm{H}, \mathrm{OCH}_{2}-\mathrm{CH}_{2}\right)$, $1.16(\mathrm{~s}, 36 \mathrm{H}, t \mathrm{Bu})$;

${ }^{7} \mathrm{Li}-\mathrm{NMR}\left(97.2 \mathrm{MHz}, \mathrm{THF}-\mathrm{D}_{8}\right): \delta 0.04$;

${ }^{13} \mathrm{C}-\mathrm{NMR}$ (125 MHz, THF-D 8 ): $\delta 113.7$ (C्C-tBu), 96.3 (br, Al-C), 68.2 (THF), 32.3 (Me), 28.8 (s, $\left.\underline{\mathrm{C}}-\mathrm{Me}_{3}\right), 26.4$ (THF);

IR (Nujol): $\widetilde{v}=2158,2112,1698,1621,1363,1247,1204,1097,1047,1031,970,910,833$, $788,744,678,632,586,534,514,406,387,354 \mathrm{~cm}^{-1}$;

EA für $\mathrm{C}_{28} \mathrm{H}_{44} \mathrm{AlLiO}$ (430.58):gef.: C 77.51, H 9.82; ber.: C 78.11, H 10.30 


\subsection{Umsetzung von Metall-Ethinyl-Verbindungen mit $\mathrm{Cp}_{2} \mathrm{ZrMe}_{2}$ \\ 4.10.1. Umsetzung von $\mathrm{Al}(\mathrm{C} \equiv \mathrm{C} t \mathrm{Bu})_{3} \cdot \operatorname{Dioxan}(15)$ mit $\mathrm{Cp}_{2} \mathrm{ZrMe}_{2}$}

Man gab zu $0.358 \mathrm{~g}(1 \mathrm{mmol}) \mathrm{Al}(\mathrm{C} \equiv \mathrm{C} t \mathrm{Bu})_{3} \cdot$ Dioxan (15) in THF (20 mL) bei RT ein Äquivalent $(0.25 \mathrm{~g}) \mathrm{Cp}_{2} \mathrm{ZrMe}_{2}$. Nach 24 Stunden Rühren wurde das Lösungsmittel im Vakuum entfernt, der Rückstand mit Toluol versetzt und der sich bildende weiße Niederschlag abfiltriert. Das Toluol des Filtrats wurde im Vakuum entfernt, mit THF versetzt und anschließend 2 Tage lang bei $12{ }^{\circ} \mathrm{C}$ gelagert. Es bildeten sich nach einigen Tagen farblose Einkristalle, die mittels einer Röntgenstrukturanalyse als $\mathrm{Cp}_{2} \mathrm{Zr}(\mathrm{C} \equiv \mathrm{CtBu})_{2}$ (20) identifiziert werden konnten. Die überstehende Lösung hatte eine gelbe Farbe. Hiervon wurden $4 \mathrm{~mL}$ abdekantiert, das Lösungsmittel im Vakuum entfernt und ein ${ }^{1} \mathrm{H}-\mathrm{NMR}$ Spektrum angefertigt.

Das Spektrum der überstehenden Lösung zeigte eine Fülle an Signalen, die nicht zugeordnet werden konnten.

Die wenigen farblosen Kristalle, die nach Entnahme für die Röntgenstrukturanalyse übrig blieben, wurden für eine NMR-Untersuchung verwendet. Für weitere spektroskopische Untersuchungen war keine Substanz mehr vorhanden.

${ }^{1} \mathrm{H}-\mathrm{NMR}\left(200.13 \mathrm{MHz}, \mathrm{C}_{6} \mathrm{D}_{6}\right): \delta 6.10(\mathrm{~s}, 10 \mathrm{H}, \mathrm{Cp}), 1.25(\mathrm{~s}, 18 \mathrm{H}, t \mathrm{Bu})$;

\subsection{Darstellung einer tetrameren Aluminium(+I)-Verbindung 4.11.1. Darstellung von $\left[\mathrm{RAII}_{2}\right]_{2}(21) \mathrm{mit} \mathrm{R}=2,6-i \mathrm{Pr}_{2} \mathrm{C}_{6} \mathrm{H}_{3} \mathrm{~N}\left(\mathrm{SiMe}_{3}\right)$}

(2,6-i $\left.\mathrm{Pr}_{2} \mathrm{C}_{6} \mathrm{H}_{3} \mathrm{~N}\left(\mathrm{SiMe}_{3}\right) \mathrm{AlMe}_{2}\right)_{2}(12.22 \mathrm{~g}, 20 \mathrm{mmol})$ in $30 \mathrm{~mL}$ Toluol gelöst, wurde langsam bei $-78{ }^{\circ} \mathrm{C}$ mit einer Lösung aus 4 Äq Iod (20.30 g, $\left.80 \mathrm{mmol}\right)$ in Toluol (200 mL) versetzt. Nach 2 h rühren ließ man die Reaktionsmischung unter Lichtausschluß langsam auf RT erwärmen und über Nacht rühren. Die leichtflüchtigen Bestandteile wurden im Vakuum entfernt und das Rohprodukt mit wenig kaltem Pentan $(75 \mathrm{~mL})$ aufgeschlämmt. Nach Filtration wurde Verbindung 21 (16.51 g, 78 \%) als farbloser Feststoff erhalten. Geeignete Kristalle für die Röntgenstrukturanalyse konnten durch langsames Entfernen des Toluols nach ca. 2 Wochen erhalten werden. 
Zers.: $167^{\circ} \mathrm{C}$;

${ }^{1} \mathrm{H}-\mathrm{NMR}\left(200.13 \mathrm{MHz}, \mathrm{C}_{6} \mathrm{D}_{6}\right): \delta 7.0-7.1$ (m, $\left.3 \mathrm{H}, \mathrm{Ar} H\right), 3.55$ (sept, $J=6.9 \mathrm{~Hz}, 2 \mathrm{H}$, $\left.\mathrm{CH}\left(\mathrm{CH}_{3}\right)_{2}\right), 1.35\left(\mathrm{~d}, J=6.9 \mathrm{~Hz}, 6 \mathrm{H}, \mathrm{CH}\left(\mathrm{CH}_{3}\right)_{2}\right), 1.22\left(\mathrm{~d}, J=6.9 \mathrm{~Hz}, 6 \mathrm{H}, \mathrm{CH}\left(\mathrm{CH}_{3}\right)_{2}\right), 0.34$ $(\mathrm{s}, 9 \mathrm{H}, t \mathrm{Bu})$;

$\mathrm{MS}(\mathrm{EI}): m / z(\%): 529$ (1/2[M], 70), 402 (1/2[M - I - H], 100);

IR (Nujol): $\widetilde{v}=1614,1554,1313,1250,1164,1101,1041,925,888,869,801,746,540 \mathrm{~cm}^{-1}$; EA für $\mathrm{C}_{30} \mathrm{H}_{52} \mathrm{Al}_{2} \mathrm{I}_{4} \mathrm{~N}_{2} \mathrm{Si}_{2}$ (1058.51): gef.: C 33.76, H 4.81, N 2.83, ber.: C 34.04, H 4.95, N $2.65 \%$.

\subsubsection{Darstellung der tetrameren Aluminium(+I)-Verbindung $[\mathrm{RAI}]_{4} \cdot \operatorname{Hexan}(22 \cdot \mathrm{Hex})$ $\left(\mathrm{R}=2,6-i \operatorname{Pr}_{2} \mathrm{C}_{6} \mathrm{H}_{3} \mathrm{~N}\left(\mathrm{SiMe}_{3}\right)\right)$}

$\mathrm{Zu} 0.53$ g (0.50 mmol) 21 wurden 4 Äq einer Na/K-Legierung, hergestellt aus Natrium (24 mg, $1 \mathrm{mmol}$ ) und Kalium (41 mg, $1 \mathrm{mmol}$ ), gegeben. Das Gemisch wurde 12 Tage lang bei RT gerührt. Nach Filtration über Celite wurde das Hexan im Vakuum entfernt. Das gelbe Rohprodukt wurde mit $4 \mathrm{~mL}$ DME versetzt und der weiße Niederschlag über Celite abfiltriert. Dann wurde die gelbe Lösung bis auf ca. $1 \mathrm{~mL}$ im Vakuum eingeengt und 30 Tage bei $12{ }^{\circ} \mathrm{C}$ im Kühlschrank gelagert. Wenige Einkristalle konnten für eine Röntgenstrukturanalyse entnommen werden. Das Lösungsmittel wurde anschließend im Vakuum entfernt, $0.7 \mathrm{~mL}$ Diisopropylether zugegeben und drei Tage bei $12{ }^{\circ} \mathrm{C}$ gelagert. 22 - Dippe konnte nachdem es auskristallisiert dann abfiltriert und getrocknet wurde, als gut löslicher, hellgelber Feststoff (96 mg, $32 \%$ ) gewonnen werden.

Aus den ${ }^{1}$ H-NMR-Daten geht hervor, dass das Produkt nach dem Zusatz von Diisopropylether nicht mehr wie im Einkristall als Hexan-Addukt, sondern nun als DiisopropyletherAddukt vorliegt.

Smp.: $270{ }^{\circ} \mathrm{C}$ (Zers.);

${ }^{1} \mathrm{H}-\mathrm{NMR}\left(200.13 \mathrm{MHz}, \mathrm{C}_{6} \mathrm{D}_{6}\right): \delta 7.05\left(\mathrm{AB}_{2}, 12 \mathrm{H}, \mathrm{Ar} H\right), 3.68\left(\mathrm{br}, 8 \mathrm{H}, \mathrm{CH}\left(\mathrm{CH}_{3}\right)_{2}\right), 3.44$ (sept, $\left.J=6.1 \mathrm{~Hz}, 2 \mathrm{H}, \mathrm{OCH}\left(\mathrm{CH}_{3}\right)_{2}\right), 1.27$ (d, br, $\left.24 \mathrm{H}, \mathrm{CH}\left(\mathrm{CH}_{3}\right)_{2}\right), 1.20$ (d, $J=6.1 \mathrm{~Hz}, 12 \mathrm{H}$, $\left.\mathrm{CH}\left(\mathrm{CH}_{3}\right)_{2}\right), 1.14$ (d, br, $\left.12 \mathrm{H}, \mathrm{CH}\left(\mathrm{CH}_{3}\right)_{2}\right), 1.06$ (d, br, $\left.12 \mathrm{H}, \mathrm{CH}\left(\mathrm{CH}_{3}\right)_{2}\right), 0.20$ (s, br, $36 \mathrm{H}$, $\left.\mathrm{Si}\left(\mathrm{CH}_{3}\right)_{3}\right)$;

Hochtemperatur- ${ }^{1} \mathrm{H}-\mathrm{NMR}\left(200.13 \mathrm{MHz}, \mathrm{C}_{6} \mathrm{D}_{6}, 70{ }^{\circ} \mathrm{C}\right): \delta 7.15\left(\mathrm{AB}_{2}, 12 \mathrm{H}, \mathrm{ArH}\right), 3.67$ (sept, $\left.J=6.9 \mathrm{~Hz}, 8 \mathrm{H}, \mathrm{CH}\left(\mathrm{CH}_{3}\right)_{2}\right), 3.45$ (sept, $\left.J=6.1 \mathrm{~Hz}, 2 \mathrm{H}, \mathrm{OCH}\left(\mathrm{CH}_{3}\right)_{2}\right), 1.24(\mathrm{~d}, J=6.9 \mathrm{~Hz}$, 
$\left.24 \mathrm{H}, \mathrm{CH}\left(\mathrm{CH}_{3}\right)_{2}\right), 1.10\left(\mathrm{~d}, J=6.9 \mathrm{~Hz}, 24 \mathrm{H}, \mathrm{CH}\left(\mathrm{CH}_{3}\right)_{2}\right), 1.06(\mathrm{~d}, J=6.1 \mathrm{~Hz}, 12 \mathrm{H}$, $\left.\mathrm{CH}\left(\mathrm{CH}_{3}\right)_{2}\right), 0.21\left(\mathrm{~s}, 27 \mathrm{H}, \mathrm{Si}\left(\mathrm{CH}_{3}\right)_{3}\right)$;

${ }^{13} \mathrm{C}-\mathrm{NMR}\left(125 \mathrm{MHz}, \mathrm{C}_{6} \mathrm{D}_{6}\right): \delta 146.2,138.8,124.7,124.1$ (arom. C), 28.1, $27.5(\mathrm{CH}), 23.8$, $23.0\left(\mathrm{CH}_{3}\right), 3.9\left(\mathrm{Si}\left(\mathrm{CH}_{3}\right)_{3}\right)$.

$\left.\mathrm{MS}(\mathrm{EI}): m / z(\%): 1100\left(\left[M^{+}\right], 16\right), 3 / 4(M, 8), 1 / 2(M), 100\right), 1 / 4(M, 15)$;

IR (Nujol): $\tilde{v}=.3176,1587,1433,1363,1314,1249,1237,1176,1103,1052,1041,884$, $836,796,749,680,539,435 \mathrm{~cm}^{-1}$;

EA für $\mathrm{C}_{66} \mathrm{H}_{118} \mathrm{Al}_{4} \mathrm{~N}_{4} \mathrm{OSi}_{4}$ (1203.96): gef.: C 64.48, H 9.09, N 4.19, ber.: C 65.84, H 9.88, $\mathrm{N} 4.65 \%$. 


\subsection{Behandlung und Entsorgung der Abfälle}

Die eingesetzten Lösungsmittel wurden abdestilliert oder im Vakuum in Kühlfallen einkondensiert und zur Entsorgung gesammelt (getrennt nach halogenfreien und halogenhaltigen Lösungsmitteln). Substanzrückstände wurden in Salzsäure gelöst und nach Neutralisation als Abfälle gesammelt.

Nicht wiederverwertbare Natriumreste wurden mit Ethanol hydrolysiert und dem Reinigungsbad für Glasgeräte zugesetzt. Dieses wurde nach Einengen als basischer Abfall entsorgt.

Die NMR-Proben wurden als Lösungsmittel entsorgt. Die bei der Anfertigung dieser Arbeit angefallenen Mengen können wie folgt eingeschätzt werden:

Halogenfreie Lösungsmittel $\quad 60 \mathrm{~L}$

Halogenhaltige Lösungsmittel $5 \mathrm{~L}$

Schwermetallabfälle $\quad 10 \mathrm{~L}$

Basenabfälle $\quad 45 \mathrm{~L}$

Säureabfälle $20 \mathrm{~L}$. 


\section{RÖNTGENOGRAPHISCHER ANHANG}

$\left[2,6-i \mathrm{Pr}_{2} \mathrm{C}_{6} \mathrm{H}_{3} \mathrm{~N}\left(\mathrm{SiMe}_{3}\right) \mathrm{AlOC}_{4} \mathrm{H}_{9}\right]_{2}$

(2)

$\left[2,6-i \mathrm{Pr}_{2} \mathrm{C}_{6} \mathrm{H}_{3} \mathrm{~N}\left(\mathrm{SiMe}_{3}\right) \mathrm{AlCl}_{2}\right]_{2}$

$\left[\mathrm{K} \cdot \mathrm{THF}\left(2,6-i \mathrm{Pr}_{2} \mathrm{C}_{6} \mathrm{H}_{3} \mathrm{~N}\left(\mathrm{SiMe}_{3}\right) \mathrm{Al}(\mathrm{C} \equiv \mathrm{CPh})_{3}\right)\right]_{2}$

$\left[\mathrm{Na} \cdot \mathrm{THF}\left(2,6-i \mathrm{Pr}_{2} \mathrm{C}_{6} \mathrm{H}_{3} \mathrm{~N}\left(\mathrm{SiMe}_{3}\right) \mathrm{Al}(\mathrm{C} \equiv \mathrm{CPh})_{3}\right)\right]_{2}$

$\left[\mathrm{Li} \cdot \operatorname{Dioxan}\left(2,6-i \mathrm{Pr}_{2} \mathrm{C}_{6} \mathrm{H}_{3} \mathrm{~N}\left(\mathrm{SiMe}_{3}\right) \mathrm{Al}(\mathrm{C} \equiv \mathrm{CPh})_{3}\right)\right]_{2} \cdot 2$ Dioxan

$\left[\mathrm{Li} \cdot 2\right.$ Dioxan $\left.\left(2,6-i \mathrm{Pr}_{2} \mathrm{C}_{6} \mathrm{H}_{3} \mathrm{~N}\left(\mathrm{SiMe}_{3}\right) \mathrm{Al}\left(\mathrm{C} \equiv \mathrm{CSiMe}_{3}\right)_{3}\right)\right] \cdot 0.75$ Dioxan

$\left[(\mathrm{Li})_{2} \cdot(\text { Dioxan })_{7}\right]_{0.5}\left[2,6-i \operatorname{Pr}_{2} \mathrm{C}_{6} \mathrm{H}_{3} \mathrm{~N}\left(\mathrm{SiMe}_{3}\right) \mathrm{Ga}\left(\mathrm{C} \equiv \mathrm{CSiMe}_{3}\right)_{3}\right] \cdot 1.5$ Dioxan

$\left[(\mathrm{Li})_{2} \cdot(\text { Dioxan })_{7}\right]_{0.5}\left[2,6-i \operatorname{Pr}_{2} \mathrm{C}_{6} \mathrm{H}_{3} \mathrm{~N}\left(\mathrm{SiMe}_{3}\right) \operatorname{In}\left(\mathrm{C} \equiv \mathrm{CSiMe}_{3}\right)_{3}\right] \cdot 1.5$ Dioxan

$\mathrm{Br}-\mathrm{Al}(\mathrm{C} \equiv \mathrm{C} t \mathrm{Bu})_{2} \cdot 2 \mathrm{THF}$

$\mathrm{Cl}-\mathrm{Ga}(\mathrm{C} \equiv \mathrm{C} t \mathrm{Bu})_{2} \cdot \mathrm{THF}$

$\mathrm{Cl}-\mathrm{In}(\mathrm{C} \equiv \mathrm{C} t \mathrm{Bu})_{2} \cdot 2 \mathrm{THF}$

$\left[\mathrm{Li} \cdot 2 \mathrm{THF}\left(2,6-i \mathrm{Pr}_{2} \mathrm{C}_{6} \mathrm{H}_{3} \mathrm{~N}\left(\mathrm{SiMe}_{3}\right) \mathrm{Ga}(\mathrm{C} \equiv \mathrm{C} t \mathrm{Bu})_{3}\right)\right]$

$\mathrm{Ga}(\mathrm{C} \equiv \mathrm{C} t \mathrm{Bu})_{3} \cdot$ Dioxan

$\mathrm{In}(\mathrm{C} \equiv \mathrm{C} t \mathrm{Bu})_{3} \cdot \mathrm{NEt}_{3} \cdot 0.5$ Hexan

$\left[\mathrm{RAlI}_{2}\right]_{2}\left(\mathrm{R}=2,6-i \mathrm{Pr}_{2} \mathrm{C}_{6} \mathrm{H}_{3} \mathrm{~N}\left(\mathrm{SiMe}_{3}\right)\right)$

$[\mathrm{RAl}]_{4} \cdot \operatorname{Hexan}\left(\mathrm{R}=2,6-i \mathrm{Pr}_{2} \mathrm{C}_{6} \mathrm{H}_{3} \mathrm{~N}\left(\mathrm{SiMe}_{3}\right)\right)$ 


\begin{tabular}{|c|c|c|}
\hline & 2 & 3 \\
\hline Summenformel & $\mathrm{C}_{19} \mathrm{H}_{36} \mathrm{AlNOSi}$ & $\mathrm{C}_{15} \mathrm{H}_{26} \mathrm{AlCl}_{2} \mathrm{NSi}$ \\
\hline $\mathrm{M}$ & 349.56 & 346.34 \\
\hline Temperatur $[\mathrm{K}]$ & $200(2)$ & $200(2)$ \\
\hline Wellenlänge [A] & 0.71073 & 0.71073 \\
\hline Kristallsystem & monoklin & monoklin \\
\hline Raumgruppe & $P 2(1) / n$ & $P 2(1) / n$ \\
\hline \multirow[t]{6}{*}{ Zelldimensionen $[\AA]$} & $a=10.0446(15)$ & $a=13.7686(16)$ \\
\hline & $b=20.741(4)$ & $b=18.803(3)$ \\
\hline & $c=10.385(2)$ & $c=15.258(3)$ \\
\hline & $\alpha=90^{\circ}$ & $\alpha=90^{\circ}$ \\
\hline & $\beta=97.136(15)^{\circ}$ & $\beta=98.075(14)^{\circ}$ \\
\hline & $\gamma=90^{\circ}$ & $\gamma=90^{\circ}$ \\
\hline Zellvolumen $\left[\AA^{3}\right]$ & $2146.7(7)$ & $3911.0(10)$ \\
\hline Berechnete Dichte $\left[\mathrm{Mg} / \mathrm{m}^{3}\right]$ & 1.082 & 1.176 \\
\hline$Z$ & 4 & 8 \\
\hline Absorptionskoeffizient $\left[\mathrm{mm}^{-1}\right]$ & 0.155 & 0.430 \\
\hline$F(000)$ & 768 & 1472 \\
\hline Kristallgröße $\left[\mathrm{mm}^{3}\right]$ & $1.0 \times 0.6 \times 0.3$ & $0.7 \times 0.5 \times 0.3$ \\
\hline Gemessener $\theta$ Bereich & 3.55 bis $25.02^{\circ}$ & 3.52 bis $25.02^{\circ}$ \\
\hline Anzahl der gemessenen Reflexe & 9121 & 9844 \\
\hline Unabhängige Reflexe & $3781[R($ int $)=0.0316]$ & $6877[R(\mathrm{int})=0.0292]$ \\
\hline Verfeinerungsmethode & $\begin{array}{l}\text { Full-matrix least-squares } \\
\text { on } F^{2}\end{array}$ & $\begin{array}{l}\text { Full-matrix least- } \\
\text { squares on } F^{2}\end{array}$ \\
\hline Daten / Restraints / Parameter & $3778 / 1 / 220$ & $6877 / 0 / 375$ \\
\hline Goodness of Fit an $F^{2}$ & 1.029 & 1.026 \\
\hline \multirow[t]{2}{*}{ Endgültige $R$-Werte $[I>2 \sigma(I)]$} & $R 1=0.0382$ & $R 1=0.0471$ \\
\hline & $w R 2=0.0974$ & $w R 2=0.1181$ \\
\hline \multirow[t]{2}{*}{$R$-Werte (sämtliche Daten) } & $R 1=0.0502$ & $R 1=0.0659$ \\
\hline & $w R 2=0.1091$ & $w R 2=0.1310$ \\
\hline $\begin{array}{l}\text { Größtes Maximum und Minimum } \\
{\left[\mathrm{e} \cdot \AA^{-3}\right]}\end{array}$ & 0.261 und -0.216 & 0.380 und -0.536 \\
\hline
\end{tabular}




\begin{tabular}{|c|c|c|}
\hline & 4 & 5 \\
\hline Summenformel & $\mathrm{C}_{86} \mathrm{H}_{98} \mathrm{Al}_{2} \mathrm{~K}_{2} \mathrm{~N}_{2} \mathrm{O}_{2} \mathrm{Si}_{2}$ & $\mathrm{C}_{86} \mathrm{H}_{98} \mathrm{Al}_{2} \mathrm{~N}_{2} \mathrm{Na}_{2} \mathrm{O}_{2} \mathrm{Si}_{2}$ \\
\hline $\mathrm{M}$ & 1380.00 & 1347.78 \\
\hline Temperatur [K] & $200(2)$ & $200(2)$ \\
\hline Wellenlänge [A] & 0.71073 & 0.71073 \\
\hline Kristallsystem & monoklin & monoklin \\
\hline Raumgruppe & $P 2(1) / n$ & $P 2(1) / n$ \\
\hline \multirow[t]{6}{*}{ Zelldimensionen $[\AA]$} & $a=12.3241(16)$ & $a=12.403(3)$ \\
\hline & $b=19.548(3)$ & $b=19.036(4)$ \\
\hline & $c=17.333(3)$ & $c=17.193(3)$ \\
\hline & $\alpha=90^{\circ}$ & $\alpha=90^{\circ}$ \\
\hline & $\beta=92.084(15)^{\circ}$ & $\beta=91.24(3)^{\circ}$ \\
\hline & $\gamma=90^{\circ}$ & $\gamma=90^{\circ}$ \\
\hline Zellvolumen $\left[\AA^{3}\right]$ & $4172.9(12)$ & $4058.4(14)$ \\
\hline Berechnete Dichte $\left[\mathrm{Mg} / \mathrm{m}^{3}\right]$ & 1.098 & 1.103 \\
\hline$Z$ & 2 & 2 \\
\hline Absorptionskoeffizient $\left[\mathrm{mm}^{-1}\right]$ & 0.208 & 0.121 \\
\hline$F(000)$ & 1472 & 1440 \\
\hline Kristallgröße $\left[\mathrm{mm}^{3}\right]$ & $1.0 \times 0.7 \times 0.6$ & $1.0 \times 0.6 \times 0.4$ \\
\hline Gemessener $\theta$ Bereich & 3.51 bis $25.05^{\circ}$ & 3.57 bis $25.09^{\circ}$ \\
\hline Anzahl der gemessenen Reflexe & 8317 & 7175 \\
\hline Unabhängige Reflexe & $7363[R($ int $)=0.1240]$ & $7175[R($ int $)=0.0000]$ \\
\hline Verfeinerungsmethode & $\begin{array}{l}\text { Full-matrix least-squares } \\
\text { on } F^{2}\end{array}$ & $\begin{array}{l}\text { Full-matrix least- } \\
\text { squares on } F^{2}\end{array}$ \\
\hline Daten / Restraints / Parameter & $7363 / 0 / 440$ & $7175 / 4 / 441$ \\
\hline Goodness of Fit an $F^{2}$ & 1.042 & 1.034 \\
\hline \multirow[t]{2}{*}{ Endgültige $R$-Werte $[I>2 \sigma(I)]$} & $R 1=0.0518$ & $R 1=0.0720$ \\
\hline & $w R 2=0.1224$ & $w R 2=0.1704$ \\
\hline \multirow[t]{2}{*}{$R$-Werte (sämtliche Daten) } & $R 1=0.0728$ & $R 1=0.1087$ \\
\hline & $w R 2=0.1405$ & $w R 2=0.2000$ \\
\hline $\begin{array}{l}\text { Größtes Maximum und Minimum } \\
{\left[\mathrm{e} \cdot \AA^{-3}\right]}\end{array}$ & 0.302 und -0.310 & 0.822 und -0.480 \\
\hline
\end{tabular}




\begin{tabular}{|c|c|c|}
\hline & 6 & 7 \\
\hline Summenformel & $\mathrm{C}_{94} \mathrm{H}_{114} \mathrm{Al}_{2} \mathrm{Li}_{2} \mathrm{~N}_{2} \mathrm{O}_{8} \mathrm{Si}_{2}$ & $\mathrm{C}_{41} \mathrm{H}_{75} \mathrm{AlLiNO}_{5.5} \mathrm{Si}_{4}$ \\
\hline $\mathrm{M}$ & 1523.89 & 816.30 \\
\hline Temperatur [K] & $203(2)$ & $200(2)$ \\
\hline Wellenlänge $[\AA]$ & 0.71073 & 0.71073 \\
\hline Kristallsystem & triklin & triklin \\
\hline Raumgruppe & $P-1$ & $P-1$ \\
\hline \multirow[t]{6}{*}{ Zelldimensionen $[\AA]$} & $a=12.216(14)$ & $a=12.024(4)$ \\
\hline & $b=13.039(18)$ & $b=19.507(8)$ \\
\hline & $c=15.551(14)$ & $c=23.550(15)$ \\
\hline & $\alpha=106.92(12)^{\circ}$ & $\alpha=96.41(6)^{\circ}$ \\
\hline & $\beta=106.731(18)^{\circ}$ & $\beta=94.38(3)^{\circ}$ \\
\hline & $\gamma=95.89(3)^{\circ}$ & $\gamma=107.574(17)^{\circ}$ \\
\hline Zellvolumen $\left[\AA^{3}\right]$ & 2220.0 & $5200.0(1)$ \\
\hline Berechnete Dichte $\left[\mathrm{Mg} / \mathrm{m}^{3}\right]$ & 1.139 & 1.043 \\
\hline$Z$ & 1 & 4 \\
\hline Absorptionskoeffizient $\left[\mathrm{mm}^{-1}\right]$ & 0.114 & 0.168 \\
\hline$F(000)$ & 816 & 1776 \\
\hline Kristallgröße $\left[\mathrm{mm}^{3}\right]$ & $1.0 \times 0.7 \times 0.7$ & $0.9 \times 0.7 \times 0.6$ \\
\hline Gemessener $\theta$ Bereich & 3.53 bis $25.01^{\circ}$ & 3.50 bis $22.52^{\circ}$ \\
\hline Anzahl der gemessenen Reflexe & 8051 & 14045 \\
\hline Unabhängige Reflexe & $7596[R($ int $)=0.1647]$ & $13532[R($ int $)=0.1123]$ \\
\hline Verfeinerungsmethode & $\begin{array}{l}\text { Full-matrix least-squares } \\
\text { on } F^{2}\end{array}$ & $\begin{array}{l}\text { Full-matrix least- } \\
\text { squares on } F^{2}\end{array}$ \\
\hline Daten / Restraints / Parameter & $7596 / 0 / 503$ & $13532 / 1397 / 1013$ \\
\hline Goodness of Fit an $F^{2}$ & 1.023 & 1.022 \\
\hline \multirow[t]{2}{*}{ Endgültige $R$-Werte $[I>2 \sigma(I)]$} & $R 1=0.0603$ & $R 1=0.0695$ \\
\hline & $w R 2=0.1614$ & $w R 2=0.1795$ \\
\hline \multirow[t]{2}{*}{$R$-Werte (sämtliche Daten) } & $R 1=0.0736$ & $R 1=0.0924$ \\
\hline & $w R 2=0.1800$ & $w R 2=0.2013$ \\
\hline $\begin{array}{l}\text { Größtes Maximum und Minimum } \\
{\left[\mathrm{e} \cdot \AA^{-3}\right]}\end{array}$ & 0.589 und -0.439 & 0.837 und -0.676 \\
\hline
\end{tabular}




\begin{tabular}{|c|c|c|}
\hline & 9 & 10 \\
\hline Summenformel & $\mathrm{C}_{50} \mathrm{H}_{93} \mathrm{GaLiNO}_{10} \mathrm{Si}_{4}$ & $\mathrm{C}_{50} \mathrm{H}_{93} \mathrm{InLiNO}{ }_{10} \mathrm{Si}_{4}$ \\
\hline $\mathrm{M}$ & 1057.27 & 1102.37 \\
\hline Temperatur $[\mathrm{K}]$ & $200(2)$ & $133(2)$ \\
\hline Wellenlänge $[\AA]$ & 0.71073 & 0.71073 \\
\hline Kristallsystem & triklin & triklin \\
\hline Raumgruppe & $P-1$ & $P-1$ \\
\hline \multirow[t]{6}{*}{ Zelldimensionen $[\AA]$} & $a=13.284(17)$ & $a=13.441(3)$ \\
\hline & $b=15.030(14)$ & $b=15.106(3)$ \\
\hline & $c=17.574(17)$ & $c=17.374(4)$ \\
\hline & $\alpha=65.19(8)^{\circ}$ & $\alpha=65.49(3)$ \\
\hline & $\beta=84.09(7)^{\circ}$ & $\beta=83.97(3)$ \\
\hline & $\gamma=79.29(7)^{\circ}$ & $\gamma=78.81(3)$ \\
\hline Zellvolumen $\left[\AA^{3}\right]$ & $3130.0(1)$ & $3147.6(11)$ \\
\hline Berechnete Dichte $\left[\mathrm{Mg} / \mathrm{m}^{3}\right]$ & 1.122 & 1.163 \\
\hline$Z$ & 2 & 2 \\
\hline Absorptionskoeffizient $\left[\mathrm{mm}^{-1}\right]$ & 0.564 & 0.499 \\
\hline$F(000)$ & 1140 & 1176 \\
\hline Kristallgröße $\left[\mathrm{mm}^{3}\right]$ & $0.7 \times 0.7 \times 0.6$ & $0.1 \times 0.2 \times 0.1$ \\
\hline Gemessener $\theta$ Bereich & 3.62 bis $20.00^{\circ}$ & 1.96 bis $24.69^{\circ}$ \\
\hline Anzahl der gemessenen Reflexe & 5964 & 58440 \\
\hline Unabhängige Reflexe & $5774[R($ int $)=0.1077]$ & $10639[R(\mathrm{int})=0.0752]$ \\
\hline Verfeinerungsmethode & $\begin{array}{l}\text { Full-matrix least-squares } \\
\text { on } F^{2}\end{array}$ & $\begin{array}{l}\text { Full-matrix least-squares } \\
\text { on } F^{2}\end{array}$ \\
\hline Daten / Restraints / Parameter & $5774 / 830 / 614$ & $10639 / 0 / 604$ \\
\hline Goodness of Fit an $F^{2}$ & 1.032 & 1.001 \\
\hline \multirow[t]{2}{*}{ Endgültige $R$-Werte $[I>2 \sigma(I)]$} & $R 1=0.0740$ & $R 1=0.0256$ \\
\hline & $w R 2=0.1897$ & $w R 2=0.0654$ \\
\hline \multirow[t]{2}{*}{$R$-Werte (sämtliche Daten) } & $R 1=0.0977$ & $R 1=0.0296$ \\
\hline & $w R 2=0.2130$ & $w R 2=0.0664$ \\
\hline $\begin{array}{l}\text { Größtes Maximum und Minimum } \\
{\left[\mathrm{e} \cdot \AA^{-3}\right]}\end{array}$ & 0.858 und -1.053 & 0.674 und -0.718 \\
\hline
\end{tabular}




\begin{tabular}{|c|c|c|}
\hline & 12 & 13 \\
\hline Summenformel & $\mathrm{C}_{20} \mathrm{H}_{34} \mathrm{AlBrO}_{2}$ & $\mathrm{C}_{16} \mathrm{H}_{26} \mathrm{ClGaO}$ \\
\hline $\mathrm{M}$ & 413.36 & 339.54 \\
\hline Temperatur $[\mathrm{K}]$ & 203(2) & $133(2) \mathrm{K}$ \\
\hline Wellenlänge $[\AA]$ & 0.71073 & $0.71073 \mathrm{~A}$ \\
\hline Kristallsystem & monoklin & monoklin \\
\hline Raumgruppe & $P 2(1) / c$ & $P 2(1) / c$ \\
\hline \multirow[t]{6}{*}{ Zelldimensionen $[\AA]$} & $a=10.278(2)$ & $a=15.162(3)$ \\
\hline & $b=12.082(2)$ & $b=11.267(2)$ \\
\hline & $c=17.997(4)$ & $c=11.592(2)$ \\
\hline & $\alpha=90^{\circ}$ & $\alpha=90^{\circ}$ \\
\hline & $\beta=91.59(3)^{\circ}$ & $\beta=111.02(3)^{\circ}$ \\
\hline & $\gamma=90^{\circ}$ & $\gamma=90^{\circ}$ \\
\hline Zellvolumen $\left[\AA^{3}\right]$ & $2233.8(8)$ & $1848.5(6)$ \\
\hline Berechnete Dichte $\left[\mathrm{Mg} / \mathrm{m}^{3}\right]$ & 1.229 & 1.220 \\
\hline$Z$ & 4 & 4 \\
\hline Absorptionskoeffizient $\left[\mathrm{mm}^{-1}\right]$ & 1.888 & 1.625 \\
\hline$F(000)$ & 872 & 712 \\
\hline Kristallgröße $\left[\mathrm{mm}^{3}\right]$ & $0.7 \times 0.7 \times 0.2$ & $0.4 \times 0.4 \times 0.3$ \\
\hline Gemessener $\theta$ Bereich & 3.56 bis $22.53^{\circ}$ & 2.31 bis $24.40^{\circ}$ \\
\hline Anzahl der gemessenen Reflexe & 2920 & 3029 \\
\hline Unabhängige Reflexe & $2902[R($ int $)=0.1261]$ & $3029[\mathrm{R}(\mathrm{int})=0.0000]$ \\
\hline Verfeinerungsmethode & $\begin{array}{l}\text { Full-matrix least- } \\
\text { squares on } F^{2}\end{array}$ & $\begin{array}{l}\text { Full-matrix least } \\
\text { squares on } F^{2}\end{array}$ \\
\hline Daten / Restraints / Parameter & $2902 / 329 / 223$ & $3029 / 190 / 224$ \\
\hline Goodness of Fit an $F^{2}$ & 1.040 & 1.009 \\
\hline \multirow[t]{2}{*}{ Endgültige $R$-Werte $[I>2 \sigma(I)]$} & $R 1=0.0809$ & $R 1=0.0368$ \\
\hline & $w R 2=0.2058$ & $w R 2=0.0919$ \\
\hline \multirow[t]{2}{*}{$R$-Werte (sämtliche Daten) } & $R 1=0.0998$ & $R 1=0.0401$ \\
\hline & $w R 2=0.2277$ & $w R 2=0.0936$ \\
\hline $\begin{array}{l}\text { Größtes Maximum und Minimum } \\
{\left[\mathrm{e} \cdot \AA^{-3}\right]}\end{array}$ & 1.689 und -1.773 & 0.602 und -0.796 \\
\hline
\end{tabular}




\begin{tabular}{|c|c|c|}
\hline & 14 & 15 \\
\hline Summenformel & $\mathrm{C}_{20} \mathrm{H}_{34} \mathrm{ClInO}_{2}$ & $\mathrm{C}_{41} \mathrm{H}_{69} \mathrm{GaLiNO}_{2} \mathrm{Si}$ \\
\hline $\mathrm{M}$ & 456.74 & 712.72 \\
\hline Temperatur $[\mathrm{K}]$ & $293(2)$ & 133(2) \\
\hline Wellenlänge $[\AA]$ & 0.71073 & 0.71073 \\
\hline Kristallsystem & monoklin & monoklin \\
\hline Raumgruppe & $P 2(1) / c$ & $P 2(1) / n$ \\
\hline \multirow[t]{6}{*}{ Zelldimensionen $[\AA]$} & $a=10.281(2)$ & $a=11.339(2)$ \\
\hline & $b=12.472(3)$ & $b=19.957(4)$ \\
\hline & $c=17.881(4)$ & $c=19.763(4)$ \\
\hline & $\alpha=90^{\circ}$ & $\alpha=90^{\circ}$ \\
\hline & $\beta=90.66(3)^{\circ}$ & $\beta=99.28(3)^{\circ}$ \\
\hline & $\gamma=90^{\circ}$ & $\gamma=90^{\circ}$ \\
\hline Zellvolumen $\left[\AA^{3}\right]$ & $2292.5(8)$ & $4413.7(15)$ \\
\hline Berechnete Dichte $\left[\mathrm{Mg} / \mathrm{m}^{3}\right]$ & 1.323 & 1.073 \\
\hline$Z$ & 4 & 4 \\
\hline Absorptionskoeffizient $\left[\mathrm{mm}^{-1}\right]$ & 1.156 & 0.681 \\
\hline$F(000)$ & 944 & 1544 \\
\hline Kristallgröße $\left[\mathrm{mm}^{3}\right]$ & $0.2 \times 0.2 \times 0.2$ & $0.3 \times 0.2 \times 0.2$ \\
\hline Gemessener $\theta$ Bereich & 1.98 bis $24.77^{\circ}$ & 1.95 bis $24.85^{\circ}$ \\
\hline Anzahl der gemessenen Reflexe & 20942 & 44187 \\
\hline Unabhängige Reflexe & $3883[R(\mathrm{int})=0.1133]$ & $7609[R($ int $)=0.0786]$ \\
\hline Verfeinerungsmethode & $\begin{array}{l}\text { Full-matrix least- } \\
\text { squares on } F^{2}\end{array}$ & $\begin{array}{l}\text { Full-matrix least squares } \\
\text { on } F^{2}\end{array}$ \\
\hline Daten / Restraints / Parameter & $3883 / 9 / 238$ & $7609 / 0 / 424$ \\
\hline Goodness of Fit an $F^{2}$ & 1.056 & 0.840 \\
\hline \multirow[t]{2}{*}{ Endgültige $R$-Werte $[I>2 \sigma(I)]$} & $R 1=0.0392$ & $R 1=0.0333$ \\
\hline & $w R 2=0.0953$ & $w R 2=0.0709$ \\
\hline \multirow[t]{2}{*}{$R$-Werte (sämtliche Daten) } & $R 1=0.0466$ & $R 1=0.0518$ \\
\hline & $w R 2=0.0977$ & $w R 2=0.0737$ \\
\hline $\begin{array}{l}\text { Größtes Maximum und Minimum } \\
{\left[\mathrm{e} \cdot \AA^{-3}\right]}\end{array}$ & 1.582 und -1.309 & 0.503 und -0.387 \\
\hline
\end{tabular}




\begin{tabular}{|c|c|c|}
\hline & 17 & $18 \cdot 0.5$ Hexan \\
\hline Summenformel & $\mathrm{C}_{22} \mathrm{H}_{35} \mathrm{GaO}_{2}$ & $\mathrm{C}_{27} \mathrm{H}_{49} \mathrm{InN}$ \\
\hline $\mathrm{M}$ & 401.22 & 502.49 \\
\hline Temperatur $[\mathrm{K}]$ & 133(2) & 133(2) \\
\hline Wellenlänge $[\AA]$ & 0.71073 & 0.71073 \\
\hline Kristallsystem & orthorhombisch & trigonal \\
\hline Raumgruppe & Pnma & $P-3 c 1$ \\
\hline \multirow[t]{6}{*}{ Zelldimensionen $[\AA]$} & $a=18.910(4)$ & $a=15.659(2)$ \\
\hline & $b=17.713(4)$ & $b=15.659(2)$ \\
\hline & $c=6.8213(14)$ & $c=14.032(3)$ \\
\hline & $\alpha=90^{\circ}$ & $\alpha=90^{\circ}$ \\
\hline & $\beta=90^{\circ}$ & $\beta=90^{\circ}$ \\
\hline & $\gamma=90^{\circ}$ & $\gamma=120^{\circ}$ \\
\hline Zellvolumen $\left[\AA^{3}\right]$ & $2284.8(8)$ & $2979.7(8)$ \\
\hline Berechnete Dichte $\left[\mathrm{Mg} / \mathrm{m}^{3}\right]$ & 1.166 & 1.120 \\
\hline$Z$ & 4 & 4 \\
\hline Absorptionskoeffizient $\left[\mathrm{mm}^{-1}\right]$ & 1.215 & 0.804 \\
\hline$F(000)$ & 856 & 1068 \\
\hline Kristallgröße $\left[\mathrm{mm}^{3}\right]$ & $0.3 \times 0.3 \times 0.2$ & $0.3 \times 0.3 \times 0.2$ \\
\hline Gemessener $\theta$ Bereich & 2.44 bis $24.79^{\circ}$ & 2.60 bis $24.82^{\circ}$ \\
\hline Anzahl der gemessenen Reflexe & 10285 & 16974 \\
\hline Unabhängige Reflexe & $2012[R($ int $)=0.0729]$ & $1712[R($ int $)=0.0583]$ \\
\hline Verfeinerungsmethode & $\begin{array}{l}\text { Full-matrix least } \\
\text { squares on } F^{2}\end{array}$ & $\begin{array}{l}\text { Full-matrix least- } \\
\text { squares on } F^{2}\end{array}$ \\
\hline Daten / Restraints / Parameter & 2012 / 0 / 159 & $1712 / 239 / 153$ \\
\hline Goodness of Fit an $F^{2}$ & 1.052 & 0.965 \\
\hline \multirow[t]{2}{*}{ Endgültige $R$-Werte $[I>2 \sigma(I)]$} & $R 1=0.0233$ & $R 1=0.0263$ \\
\hline & $w R 2=0.0687$ & $w R 2=0.0687$ \\
\hline \multirow[t]{2}{*}{$R$-Werte (sämtliche Daten) } & $R 1=0.0261$ & $R 1=0.0416$ \\
\hline & $w R 2=0.0699$ & $w R 2=0.0735$ \\
\hline $\begin{array}{l}\text { Größtes Maximum und Minimum } \\
{\left[\mathrm{e} \cdot \AA^{-3}\right]}\end{array}$ & 0.369 und -0.464 & 0.486 und -0.287 \\
\hline
\end{tabular}




\begin{tabular}{|c|c|c|}
\hline & 20 & 21 \\
\hline Summenformel & $\mathrm{C}_{22} \mathrm{H}_{28} \mathrm{Zr}$ & $\mathrm{C}_{15} \mathrm{H}_{26} \mathrm{AlI}_{2} \mathrm{NSi}$ \\
\hline $\mathrm{M}$ & 383.66 & 529.24 \\
\hline Temperatur $[\mathrm{K}]$ & $200(2)$ & $200(2)$ \\
\hline Wellenlänge $[\AA]$ & 0.71073 & 0.71073 \\
\hline Kristallsystem & monoklin & monoklin \\
\hline Raumgruppe & $C 2 / c$ & $P 2(1) / c$ \\
\hline \multirow[t]{6}{*}{ Zelldimensionen $[\AA]$} & $a=24.860(5)$ & $a=12.957(5)$ \\
\hline & $b=6.1662(12)$ & $b=9.282(2)$ \\
\hline & $c=16.553(3)$ & $c=17.541(14)$ \\
\hline & $\alpha=90^{\circ}$ & $\alpha=90^{\circ}$ \\
\hline & $\beta=127.11(3)^{\circ}$ & $\beta=96.21(5)^{\circ}$ \\
\hline & $\gamma=90^{\circ}$ & $\gamma=90^{\circ}$ \\
\hline Zellvolumen $\left[\AA^{3}\right]$ & $2023.5(7)$ & $2100.0(1)$ \\
\hline Berechnete Dichte $\left[\mathrm{Mg} / \mathrm{m}^{3}\right]$ & 1.259 & 1.676 \\
\hline$Z$ & 4 & 4 \\
\hline Absorptionskoeffizient $\left[\mathrm{mm}^{-1}\right]$ & 0.542 & 3.091 \\
\hline$F(000)$ & 800 & 1024 \\
\hline Kristallgröße $\left[\mathrm{mm}^{3}\right]$ & $1.0 \times 0.5 \times 0.3$ & $1.00 \times 0.70 \times 0.20$ \\
\hline Gemessener $\theta$ Bereich & 3.53 bis $24.99^{\circ}$ & 3.69 bis $22.51^{\circ}$ \\
\hline Anzahl der gemessenen Reflexe & 1845 & 2784 \\
\hline Unabhängige Reflexe & $1782[R($ int $)=0.0681]$ & $2734[R($ int $)=0.0350]$ \\
\hline Verfeinerungsmethode & $\begin{array}{l}\text { Full-matrix least- } \\
\text { squares on } F^{2}\end{array}$ & $\begin{array}{l}\text { Full-matrix least- } \\
\text { squares on } F^{2}\end{array}$ \\
\hline Daten / Restraints / Parameter & $1782 / 0 / 105$ & $2734 / 0 / 188$ \\
\hline Goodness of Fit an $F^{2}$ & 1.071 & 1.113 \\
\hline \multirow[t]{2}{*}{ Endgültige $R$-Werte $[I>2 \sigma(I)]$} & $R 1=0.0368$ & $R 1=0.0539$ \\
\hline & $w R 2=0.0969$ & $w R 2=0.1385$ \\
\hline \multirow[t]{2}{*}{$R$-Werte (sämtliche Daten) } & $R 1=0.0396$ & $R 1=0.0602$ \\
\hline & $w R 2=0.1010$ & $w R 2=0.1463$ \\
\hline $\begin{array}{l}\text { Größtes Maximum und Minimum } \\
{\left[\mathrm{e} \cdot \AA^{-3}\right]}\end{array}$ & 0.623 und -1.633 & 1.456 und -2.245 \\
\hline
\end{tabular}




\begin{tabular}{|c|c|}
\hline & 22 \\
\hline Summenformel & $\mathrm{C}_{66} \mathrm{H}_{118} \mathrm{Al}_{4} \mathrm{~N}_{4} \mathrm{Si}_{4}$ \\
\hline $\mathrm{M}$ & 1187.92 \\
\hline Temperatur $[\mathrm{K}]$ & $133(2)$ \\
\hline Wellenlänge $[\AA]$ & 0.71073 \\
\hline Kristallsystem & triklin \\
\hline Raumgruppe & $P-1$ \\
\hline \multirow[t]{6}{*}{ Zelldimensionen $[\AA]$} & $a=13.0801(9)$ \\
\hline & $b=13.7352(10)$ \\
\hline & $c=20.8205(15)$ \\
\hline & $\alpha=83.173(6)^{\circ}$ \\
\hline & $\beta=85.513(6)^{\circ}$ \\
\hline & $\gamma=81.883(6)^{\circ}$ \\
\hline Zellvolumen $\left[\AA^{3}\right]$ & $3669.6(5)$ \\
\hline Berechnete Dichte $\left[\mathrm{Mg} / \mathrm{m}^{3}\right]$ & 1.075 \\
\hline$Z$ & 2 \\
\hline Absorptionskoeffizient $\left[\mathrm{mm}^{-1}\right]$ & 0.167 \\
\hline$F(000)$ & 1300 \\
\hline Kristallgröße $\left[\mathrm{mm}^{3}\right]$ & $0.3 \times 0.2 \times 0.3$ \\
\hline Gemessener $\theta$ Bereich & 1.58 bis $24.83^{\circ}$ \\
\hline Anzahl der gemessenen Reflexe & 36000 \\
\hline Unabhängige Reflexe & $\begin{array}{l}12430[R(\text { int })= \\
0.0833]\end{array}$ \\
\hline Verfeinerungsmethode & $\begin{array}{l}\text { Full-matrix least } \\
\text { squares on } F^{2}\end{array}$ \\
\hline Daten / Restraints / Parameter & $12430 / 0 / 731$ \\
\hline Goodness of Fit an $F^{2}$ & 1.039 \\
\hline \multirow[t]{2}{*}{ Endgültige $R$-Werte $[I>2 \sigma(I)]$} & $R 1=0.0492$ \\
\hline & $w R 2=0.1153$ \\
\hline \multirow[t]{2}{*}{$R$-Werte (sämtliche Daten) } & $R 1=0.0714$ \\
\hline & $w R 2=0.1209$ \\
\hline $\begin{array}{l}\text { Größtes Maximum und Minimum } \\
{\left[\mathrm{e} \cdot \AA^{-3}\right]}\end{array}$ & 0.926 und -0.522 \\
\hline
\end{tabular}




\section{Literaturverzeichnis}

[1] Autorenkollektiv, Organikum, 16. Auflage, V.E.B. Deutscher Verlag der Wissenschaften, Berlin, 1986.

[2] K. P. C. Vollhardt, N. E. Schore, Organische Chemie, 3. Auflage, Wiley- VCH, Weinheim, 2000.

[3] F. A. Carey, R. J. Sundberg, Organische Chemie, 1. Auflage, Wiley- VCH, Weinheim, 1995.

[4] N. A. Petasis, E. I. Bzowej, J. Am. Chem. Soc. 1990, 112, 6392.

[5] F. N. Tebbe, G. W. Parshall, G. S. Reddy, J. Am. Chem. Soc. 1978, 100, 3611.

[6] C. Elschenbroich, A. Salzer, Organometallchemie, 3. Aufl., Teubner, Stuttgart, 1990.

[7] S. H. Pine, R. Zahler, D. A. Evans, R. H. Grubbs, J. Am. Chem. Soc. 1980, 102, 3270.

[8] L. F. Cannizzo, R. H. Grubbs, J. Org. Chem. 1985, 50, 2316.

[9] S. L. Buchwald, R. H. Grubbs, J. Am. Chem. Soc. 1983, 105, 5490.

[10] Autorenkollektiv, The Chemistry of Ketenes, Allenes, and Related Compounds, Hrsg.: S. Patai, Wiley-Interscience, New York, 1980.

[11] G. Erker, M. Albrecht, C. Krüger, M. Nolte, S. Werner, Organometallics 1993, 12, 4979.

[12] G. Erker, M. Albrecht, C. Krüger, S. Werner, Organometallics 1991, 10, 3791.

[13] G. D. Stucky, A. M. McPherson, W. E. Rhine, J. J. Eisch, J. L. Considine, J. Am. Chem. Soc. 1974, 96, 1941.

[14] W. Zheng, N. C. Mösch-Zanetti, H. W. Roesky, M. Hewitt, F. Cimpoesu, T. R. Schneider, A. Stasch, J. Prust, Angew. Chem. 2000, 112, 3229; Angew. Chem. Int. Ed. 2000, 39, 3099.

[15] R. J. Wehmschulte, P. P. Power, Inorg. Chem. 1994, 33, 5611.

[16] R. J. Wehmschulte, P. P. Power, Polyhedron 2000, 19, 1649.

[17] C. Cui, H. W. Roesky, M. Noltemeyer, H.-G. Schmidt, Inorg. Chem. 2000, 39, 3678.

[18] J. Weidlein, U. Müller, K. Dehnicke, Schwingungsfrequenzen, Bd. I, 1. Auflage, GThieme, Stuttgart, 1981.

[19] C. Schnitter, Dissertation, Universität Göttingen 1998.

[20] C. Böker, M. Noltemeyer, H. Gornitzka, B. O. Kneisel, M. Teichert, R. Herbst-Irmer, A. Meller, Main Group Met. Chem. 1998, 21, 565.

[21] B.-T. Ko, C.-C. Wu, C.-C. Lin, Organometallics 2000, 19, 1864. 
[22] J. A. Francis, C. N. McMahon, S. G. Bott, A. R. Barron, Organometallics 1999, 18, 4399.

[23] M. L. Sierra, V. S. J. de Mel, J. P. Oliver, Organometallics 1989, 8, 2486.

[24] A. V. Korolev, I. A. Guzei, R. F. Jordan, J. Am. Chem. Soc. 1999, 121, 11605.

[25] C.-H. Lin, B.-T. Ko, F.-C. Wang, C.-C. Lin, C.-Y. Kuo, J. Organomet. Chem. 1999, $575,67$.

[26] G. L. Wood, C. B. Knobler, M. F. Hawthorne, Inorg. Chem. 1989, $28,382$.

[27] D. G. Sekutowski, G. D. Stucky, J. Am. Chem. Soc. 1976, 98, 1376.

[28] J. H. Teuben, H. J. de Liefde Meijer, J. Organomet. Chem. 1969, 17, 87.

[29] M. F. Lappert, C. L. Raston, J. Chem. Soc., Chem. Comm. 1980, 1284.

[30] M. F. Lappert, C. J. Pickett, P. I. Riley, P. I. W. Yarrow, J. Chem. Soc., Dalton Trans. $1981,805$.

[31] Autorenkollektiv, Comprehensive Organometallic Chemistry, (Hrsg. G. Wilkinson, F. G. A. Stone, E. W. Abel), Bd. 7, Pergamon Press, Oxford, 1982.

[32] B. Temme, G. Erker, R. Fröhlich, M. Grehl, Angew. Chem. 1994, 106, 1570; Angew. Chem. Int. Ed. Engl. 1994, 33, 1480.

[33] P.-M. Pellney, F. G. Kirchbauer, V. V. Burlakov, W. Baumann, A. Spannenberg, U. Rosenthal, J. Am. Chem. Soc. 1999, 121, 8313.

[34] K. Clauss, H. Bestian, Justus Liebigs Ann. Chem. 1962, 656, 8.

[35] E. Samuel, M. D. Rausch, J. Am. Chem. Soc. 1973, 95, 6263.

[36] Autorenkollektiv, Houben-Weyl: Methoden der Organischen Chemie, Thieme, Stuttgart, 4. Aufl., 13/4, 1970 (und darin angegebene Literaturstellen).

[37] T. Mole, J. R. Surtees, Aust. J. Chem. 1964, 17, 1229.

[38] J. K. Ruff, J. Am. Chem. Soc. 1961, 83, 1798.

[39] P. Binger, Angew. Chem. 1963, 75, 918.

[40] R. J. Wehmschulte, W. J. Grigsby, B. Schiemenz, R. A. Bartlett, P. P. Power, Inorg. Chem. 1996, 35, 6694.

[41] G. Allegra, G. Perego, A. Immirzi, Makromol. Chem. 1963, 61, 69.

[42] W. Uhl, A. Vester, W. Hiller, Z. Anorg. Allg. Chem. 1990, 589, 175.

[43] J. C. Ma, D. A. Dougherty, Chem. Rev. 1997, 97, 1303.

[44] G. W. Gokel, S. L. De Wall, E. S. Meadows, Eur. J. Org. Chem. 2000, 2967.

[45] L. Bonomo, E. Solari, R. Scopelliti, C. Floriani, Chem. Eur. J. 2001, 7, 1322.

[46] J. D. Smith, Adv. Organomet. Chem. 1998, 43, 267. 
[47] C. Lambert, P. v. Ragué Schleyer, Angew. Chem. 1994, 106, 1187; Angew. Chem. Int. Ed. Engl. 1994, 33, 1129.

[48] W. J. Evans, R. A. Keyer, J. W. Ziller, Organometallics 1993, 12, 2618.

[49] V. Varga, J. Hiller, M. Polášek, U. Thewalt, K. Mach, J. Organomet. Chem. 1996, 515, 57.

[50] V. Varga, J. Hiller, U. Thewalt, M. Polášek, K. Mach, J. Organomet. Chem. 1998, 553, 15.

[51] M. Geissler, J. Kopf, E. Weiss, Chem. Ber. 1989, 122, 1395.

[52] J. Hiller, V. Varga, U. Thewalt, K. Mach, Coll. Czech. Chem. Commun. 1997, 62, 1446.

[53] M. Karl, G. Seybert, W. Massa, K. Harms, S. Agarwal, R. Maleika, W. Stelter, A. Greiner, W. Heitz, B. Neumüller, K. Dehnicke, Z. Anorg. Allg. Chem. 1999, 625, 1301.

[54] Holleman-Wiberg, Lehrbuch der Anorganischen Chemie, 101. Aufl., de Gruyter, Berlin, $1995,248$.

[55] M. Hesse, H. Meier, B. Zeeh, Spektroskopische Methoden in der organischen Chemie, 4. Aufl., G- Thieme, Stuttgart, 1991, 70.

[56] J. Prust, P. Müller, C. Rennekamp, H. W. Roesky, I. Usón, J. Chem. Soc., Dalton Trans. 1999, 2265.

[57] A. L. Balch, L. Latos-Grażyński, B. C. Noll, S. L. Phillips, Inorg. Chem. 1993, 32, 1124.

[58] K. E. Lee, K. T. Higa, R. A. Nissan, R. J. Butcher, Organometallics 1992, 11, 2817.

[59] B. Tecle, W. H. Ilsley, J. P. Oliver, Inorg. Chem. 1981, 20, 2335.

[60] W. Uhl, T. Spies, Z. Anorg. Allg. Chem. 2000, 626, 1059.

[61] A. J. Edwards, A. Fallaize, P. R. Raithby, M.-A. Rennie, A. Steiner, K. L. Verhorevoort, D. S. Wright, J. Chem. Soc., Dalton Trans. 1996, 133.

[62] H. Schumann, T. D. Seuß, O. Just, R. Weimann, H. Hemling, F. H. Görlitz, J. Organomet. Chem. 1994, 479, 171.

[63] J. Prust, Dissertation, Universität Göttingen 2001.

[64] W. Fries, W. Schwarz, H. D. Hausen, J. Weidlein, J. Organomet. Chem. 1978, 159, 373.

[65] J. Fujiwara, H. Sano, K. Maruoka, H. Yamamoto, Tetrahedron Lett. 1984, 25, 2367.

[66] G. B. Reddy, T. Hanamoto, T. Hiyama, Tetrahedron Lett. 1991, 32, 521.

[67] I. Pérez, J. P. Sestelo, M. A. Maestro, A. Mouriño, L. A. Sarandeses, J. Org. Chem. 1998, 63, 10074 .

[68] I. Pérez, J. P. Sestelo, L. A. Sarandeses, Org. Lett. 1999, 1, 1267.

[69] A. H. Cowley, M. C. Cushner, R. E. Davis, P. E. Riley, Inorg. Chem. 1981, 20, 1179. 
[70] N. L. Pickett, O. Just, X. Li, D. G. Vanderveer, W. S. Rees, Jr., J. Organomet. Chem. 1999, 582, 119.

[71] J. Vanderhooft, R. D. Ernst, F. W. Cagle, Jr., R. J. Neustadt, Inorg. Chem. 1982, 21, 1876.

[72] H. A. Bent, Chem. Rev. 1968, 68, 587.

[73] H. Demarne, P. Cadiot, Bull. Soc. Chim. Fr. 1968, 1, 211.

[74] N. D. Reddy, H. W. Roesky (unveröffentlicht)

[75] W. Zheng, H. W. Roesky, J. Chem. Soc., Dalton Trans. 2002, 2787.

[76] H. Sussek, O. Stark, A. Devi, H. Pritzkow, R. A. Fischer, J. Organomet. Chem. 2000, $602,29$.

[77] H. Schumann, U. Hartmann, W. Wassermann, A. Dietrich, F. H. Görlitz, L. Pohl, M. Hostalek, Chem. Ber. 1990, 123, 2093.

[78] W. Ahlers, B. Temme, G. Erker, R. Fröhlich, T. Fox, J. Organomet. Chem. 1997, 527, 191.

[79] G. Erker, W. Frömberg, R. Benn, R. Mynott, K. Angermund, C. Krüger, Organometallics 1989, 8, 911.

[80] A. Ahmed, W. Schwarz, H. Hess, Z. Naturforsch. 1978, 33b, 43.

[81] I. Krossing, H. Nöth, H. Schwenk-Kircher, Eur. J. Inorg. Chem. 1998, 927.

[82] C. Schnitter, K. Klimek, H. W. Roesky, T. Albers, H.-G. Schmidt, C. Röpken, E. Parisini, Organometallics 1998, 17, 2249.

[83] A. Ecker, R. Köppe, C. Üffing, H. Schnöckel, Z. Anorg. Allg. Chem. 1998, 624, 817.

[84] C. Üffing, E. Baum, R. Köppe, H. Schnöckel, Angew. Chem. 1998, 110, 2488; Angew. Chem. Int. Ed. 1998, 37, 2397.

[85] A. Ecker, E. Baum, M. A. Friesen, M. A. Junker, C. Üffing, R. Köppe, H. Schnöckel, Z. Anorg. Allg. Chem. 1998, 624, 513.

[86] P. Bissinger, P. Mikulcik, J. Riede, A. Schier, H. Schmidbaur, J. Organomet. Chem. 1993, 446, 37.

[87] R. A. Fischer, J. Weiß, Angew. Chem. 1999, 111, 3003; Angew. Chem. Int. Ed. 1999, $38,2830$.

[88] C. Cui, H. W. Roesky, H.-G. Schmidt, M. Noltemeyer, H. Hao, F. Cimpoesu, Angew. Chem. 2000, 112, 4444; Angew. Chem. Int. Ed. 2000, 39, 4274.

[89] C. Dohmeier, C. Robl, M. Tacke, H. Schnöckel, Angew. Chem. 1991, 103, 594; Angew. Chem. Int. Ed. Engl. 1991, 30, 564. 
[90] C. Schnitter, H. W. Roesky, C. Röpken, R. Herbst-Irmer, H.-G. Schmidt, M. Noltemeyer, Angew. Chem. 1998, 110, 2059; Angew. Chem. Int. Ed. 1998, 37, 1952.

[91] H. Sitzmann, M. F. Lappert, C. Dohmeier, C. Üffing, H. Schnöckel, J. Organomet. Chem. 1998, 561, 203.

[92] A. Purath, C. Dohmeier, A. Ecker, H. Schnöckel, K. Amelunxen, T. Passler, N. Wiberg, Organometallics 1998, 17, 1894.

[93] A. Purath, H. Schnöckel, J. Organomet. Chem. 1999, 579, 373.

[94] N. Wiberg, T. Blank, W. Kaim, B. Schwederski, G. Linti, Eur. J. Inorg. Chem. 2000, 1475.

[95] A. Purath, R. Köppe, H. Schnöckel, Angew. Chem. 1999, 111, 3114; Angew. Chem. Int. Ed. 1999, 38, 2926.

[96] A. Purath, R. Köppe, H. Schnöckel, Chem. Commun. 1999, 1933.

[97] W. Hiller, K.-W. Klinkhammer, W. Uhl, J. Wagner, Angew. Chem. 1991, 103, 182; Angew. Chem. Int. Ed. Engl. 1991, 30, 179.

[98] A. Purath, C. Dohmeier, A. Ecker, R. Köppe, H. Krautscheid, H. Schnöckel, R. Ahlrichs, C. Stoermer, J. Friedrich, P. Jutzi, J. Am. Chem. Soc. 2000, 122, 6955.

[99] H. Köhnlein, G. Stößer, E. Baum, E. Möllhausen, U. Huniar, H. Schnöckel, Angew. Chem. 2000, 112, 828; Angew. Chem. Int. Ed. 2000, 39, 799.

[100] C. Klemp, R. Köppe, E. Weckert, H. Schnöckel, Angew. Chem. 1999, 111, 1852; Angew. Chem. Int. Ed. 1999, 38, 1740.

[101] H. Köhnlein, A. Purath, C. Klemp, E. Baum, I. Krossing, G. Stösser, H. Schnöckel, Inorg. Chem. 2001, 40, 4830.

[102] A. Ecker, E. Weckert, H. Schnöckel, Nature 1997, 387, 379.

[103] T. Kottke, D. Stalke, J. Appl. Crystallogr. 1993, 26, 615.

[104] G. M. Sheldrick, SHELXS-97, Program for Crystal Structure Solution, Göttingen, 1997.

[105] G. M. Sheldrick, SHELXL-97, Program for Crystal Structure Refinement, Göttingen, 1997.

[106] S. D. Waezsada, F.-Q. Liu, E. F. Murphy, H. W. Roesky, M. Teichert, I. Usón, H.-G. Schmidt, T. Albers, E. Parisini, M. Noltemeyer, Organometallics 1997, 16, 1260.

[107] H. Köpf, M. Schmidt, J. Organomet. Chem. 1967, 10, 383.

[108] H. Lang, D. Seyferth, Z. Naturforsch. 1990, 45b, 212.

[109] J. M. Manriquez, D. R. McAlister, E. Rosenberg, A. M. Shiller, K. L. Williamson, S. I. Chan, J. E. Bercaw, J. Am. Chem. Soc. 1978, 100, 3078. 
[110] J. M. Manriquez, J. E. Bercaw, J. Am. Chem. Soc. 1974, 96, 6229. 


\section{LEBENSLAUF}

\section{Marcus Schiefer}

geboren am 22.01.1973 in Hamburg

Eltern : $\quad$ Dr. med. Carsten Schiefer

Veronika Schiefer, geb. Hamann

Familienstand : ledig

Staatsangehörigkeit : deutsch

SCHUlische AusbiLdung:

1979 - 1983 Grundschule am Bahnhof in Bad Bramstedt

1983 - 1993 Jürgen-Fuhlendorf-Schule Bad Bramstedt

$05.93 \quad$ Abitur

Universitäre AUSBILdung:

10.93 Immatrikulation an der Georg-August-Universität Göttingen

06.96 Diplomvorprüfung in Chemie

10.98 - 07.99 Diplomarbeit unter Anleitung von Prof. Dr. Dr. h.c. mult. H. W. Roesky bei der Bayer AG in Leverkusen

07.99 Diplomhauptprüfung Chemie

09.99 - 10.02 Anfertigung der vorliegenden Dissertation im Arbeitskreis von Prof. Dr. Dr. h.c. mult. H. W. Roesky am Institut für Anorganische Chemie der GeorgAugust-Universität Göttingen 